Supporting Information

\title{
Cobalt-Catalyzed Remote Hydroboration of Alkenyl Amines
}

Yaqin Lei, Jiaxin Huang, and Wanxiang Zhao*

State Key Laboratory of Chemo/Biosensing and Chemometrics, College of Chemistry and Chemical Engineering, Hunan University, Changsha, Hunan 410082, China.

E-mail: zhaowanxiang@hnu.edu.cn.

\section{Table of Contents}

1. General Information ........................................................................ 2

2. Synthesis of Substrates ........................................................ 3

3. Co-Catalyzed Remote Hydroboration of Alkenyl Amines...... 19

4. Gram-scale Reaction and Applications................................... 35

5. Control Experiments ............................................................. 39

6. References ................................................................................ 42

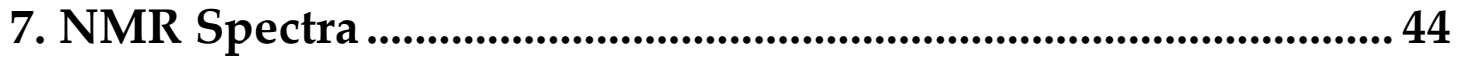




\section{General Information}

Unless otherwise noted, all reactions were conducted in oven-dried vials with a magnetic stir bar under nitrogen atmosphere. Solvents were purified under nitrogen using a solvent purification system. 2,2':6',2'-Terpyridine (tpy), 4'-(4-pyridyl)-2,2':6',2"-terpyridine L5, bipyridine (bpy), 1,2-bis(dicyclohexylphosphino)ethane (dcype), bases and pinacolborane (HBpin) were purchased from Energy Chemicals Inc. or Macklin Inc. and used as received. Co(acac)2 (98\%, GD250261, Energy Chemicals Inc.), Co(acac)3 (98\%, EF240062, Energy Chemicals Inc.), $\mathrm{CoCl}_{2}$ (98\%, GE070006, Energy Chemicals Inc.), $\mathrm{CoBr}_{2}$ (97\%, FL200068, Energy Chemicals Inc.) and $\mathrm{Co}(\mathrm{OAc})_{2}$ (98\%, KYEK893, Innochem Inc.) were commercial available and used as received. Ligands, L1-L4 and L6 were prepared according to the reported procedure. ${ }^{1}$ Analytical thin layer chromatography (TLC) was performed using silica gel plates. Visualisation was by ultraviolet fluorescence, and/or phosphomolybdic acid, and/or $\mathrm{KMnO}_{4}$. Flash column chromatography was performed using EM Science (200-300 mesh) silica gel.

${ }^{1} \mathrm{H}-\mathrm{Nuclear}$ Magnetic Resonance ( ${ }^{1} \mathrm{H}$ NMR) and ${ }^{13} \mathrm{C}$ Nuclear Magnetic Resonance $\left({ }^{13} \mathrm{C}\right.$ NMR) spectra were recorded on Bruker $400 \mathrm{MHz}$ at $20^{\circ} \mathrm{C}$ with $\mathrm{CDCl}_{3}$ as solvent, and were reported in ppm referenced to tetramethylsilane $\left(\delta=7.26 \mathrm{ppm}\right.$ for $\left.{ }^{1} \mathrm{H}-\mathrm{NMR}\right)$ and $\mathrm{CDCl}_{3}(\delta=$ $77.16 \mathrm{ppm}$ for $\left.{ }^{13} \mathrm{C}-\mathrm{NMR}\right)$. The data are reported as follows: chemical shift (ppm), multiplicity $\left(\mathrm{s}=\right.$ singlet, $\mathrm{d}=$ doublet, $\mathrm{dd}=$ doublet of doublet, $\mathrm{t}=$ triplet, $\mathrm{m}=$ multiplet, $\mathrm{m}_{\mathrm{c}}=$ centered multiplet, $\mathrm{br}=$ broad), coupling constant $J(\mathrm{~Hz})$, and integration. High resolution mass spectra were recorded on a Bruker Maxis System using Electron Spray Ionization (ESI). IR spectra were collected on a Spectrum QATR-S from SHIMADZU and reported in unit of $\mathrm{cm}^{-1}$. 


\section{Synthesis of Substrates}

$\mathbf{1 i}^{2}, \mathbf{1} \mathbf{k}^{3}, \mathbf{1 1}^{4}, \mathbf{1} \mathbf{m}^{5}, \mathbf{1} \mathbf{n}^{6}, \mathbf{1} \mathbf{u}^{7}$ and $\mathbf{1} \mathbf{a i}^{8}$ were prepared according to the reported procedures.

\section{General Procedure A}

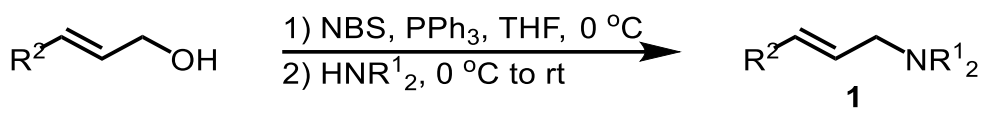

Following the literature procedure ${ }^{9}$, a solution of allylic alcohol (1.0 equiv.) and $\mathrm{PPh}_{3}(1.0$ equiv.) in THF $(0.5 \mathrm{M})$ at $0{ }^{\circ} \mathrm{C}$ was treated with NBS (1 equiv.) in 5 portions. After $1 \mathrm{~h}$, the corresponding amine (1.5 equiv.) was added at $0{ }^{\circ} \mathrm{C}$. The reaction was allowed to warm to room temperature and stirred for additional $12 \mathrm{~h}$. The mixture was diluted with $\mathrm{Et}{ }_{2} \mathrm{O}(0.2 \mathrm{M})$ and stirred under air for additional $15 \mathrm{~min}$. Then the mixture was filtered through celite and washed with $\mathrm{Et}_{2} \mathrm{O}$. The filtrate was concentrated under reduced pressure, and the crude residue was purified by column chromatography to yield the corresponding allylic amine.

\section{General Procedure B}

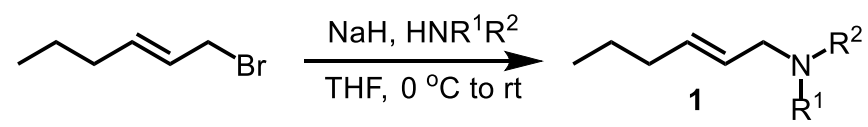

Following the literature procedure ${ }^{10}$, to a suspension of $\mathrm{NaH}$ (1.5 equiv) and amine (1.0 euiqv) in THF $(0.25 \mathrm{M})$ was added $(E)$-1-bromohex-2-ene (1.2 equiv) at $0{ }^{\circ} \mathrm{C}$. The reaction mixture was then warmed to room temperature and stirred until TLC indicating reaction completed. The reaction was then quenched with $\mathrm{H}_{2} \mathrm{O}$ and extracted with ethyl acetate. The combined organic layers were washed with brine, dried over $\mathrm{Na}_{2} \mathrm{SO}_{4}$, and concentrated. The crude residue was purified by flash column chromatography to give the corresponding allylic amine.

\section{General Procedure C}

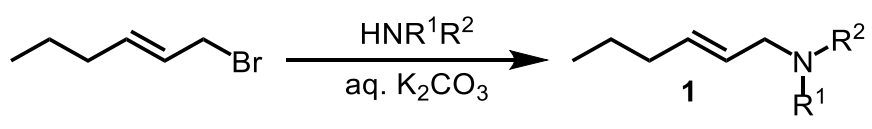

The amine (1.0 euiqv.) was added to the $(E)$-1-bromohex-2-ene (2.0 equiv) in saturated aqueous $\mathrm{K}_{2} \mathrm{CO}_{3}(2.6 \mathrm{M})$. The reaction mixture was stirred overnight. The reaction was then quenched with $\mathrm{H}_{2} \mathrm{O}$ and extracted with ethyl acetate. The combined organic layers were 
washed with brine, dried over $\mathrm{Na}_{2} \mathrm{SO}_{4}$, and concentrated. The crude residue was purified by flash column chromatography to give the corresponding allylic amine.

\section{General Procedure D:}

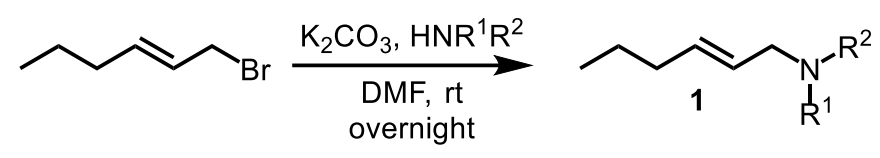

Following the literature procedure ${ }^{11}$, a mixture of amine (1.0 equiv), (E)-1-bromohex-2ene (1.0 equiv) and $\mathrm{K}_{2} \mathrm{CO}_{3}$ (3.0 equiv) in DMF $(0.6 \mathrm{M})$ was stirred for $12 \mathrm{~h}$ at room temperature, and water and ethyl acetate were then added. The aqueous phase was separated and extracted with ethyl acetate. The combined organic phases were washed with brine, dried with $\mathrm{Na}_{2} \mathrm{SO}_{4}$ and concentrated in vacuo. The residue was purified by column chromatography to give the corresponding allylic amine.

\section{General Procedure E:}

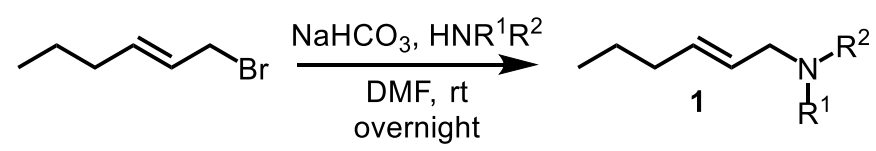

Following the literature procedure ${ }^{12}$, a mixture of amine (1.0 equiv), (E)-1-bromohex-2ene (1.0 equiv) and $\mathrm{NaHCO}_{3}$ (1.5 equiv) in $\operatorname{DMF}(0.25 \mathrm{M})$ was stirred for $12 \mathrm{~h}$ at room temperature, and water and ethyl acetate were then added. The aqueous phase was separated and extracted with ethyl acetate. The combined organic phase was washed with brine, dried with $\mathrm{Na}_{2} \mathrm{SO}_{4}$ and concentrated in vacuo. The residue was purified by column chromatography to give the corresponding allylic amines.

\section{General Procedure F:}

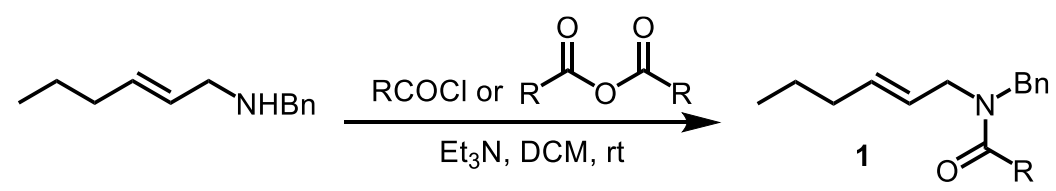

To a solution of (E)-N-benzylhex-2-en-1-amine (1.0 equiv) and $\mathrm{Et}_{3} \mathrm{~N}$ (1.2-1.5 equiv) in $\mathrm{CH}_{2} \mathrm{Cl}_{2}$ was added acyl chloride or anhydride (0.9-1.3 equiv) at $0{ }^{\circ} \mathrm{C}$. The resulting mixture was then stirred for $3 \mathrm{~h}$, and then quenched with $\mathrm{H}_{2} \mathrm{O}$ at room temperature, extracted with $\mathrm{CH}_{2} \mathrm{Cl}_{2}$, dried with $\mathrm{Na}_{2} \mathrm{SO}_{4}$, filtered and concentrated. The residue was purified by silica gel flash chromatography to afford corresponding allylic amine. 


\section{General Procedure G:}

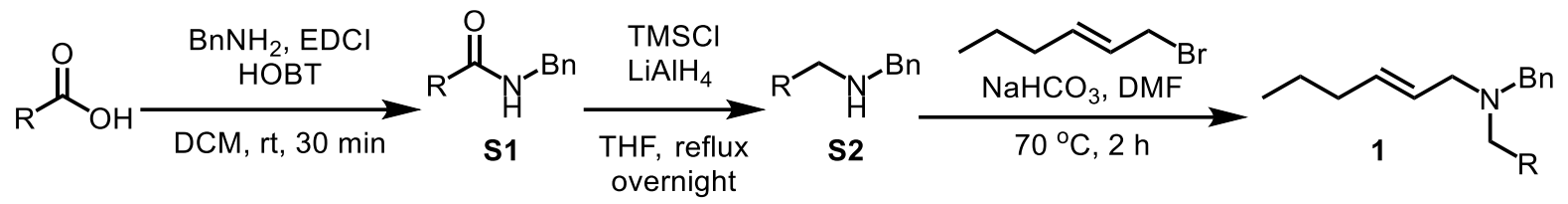

Following the literature procedure ${ }^{13}$, to a solution of acid (1.0 equiv) in DCM $(0.15 \mathrm{M})$ was added benzylamine (1.5 equiv), HOBT (1.5 equiv), and EDCI (1.5 equiv) in sequence at room temperature. After stirring for $30 \mathrm{~min}$, the mixture was diluted with saturated aqueous $\mathrm{NaHCO}_{3}$ solution, extracted with $\mathrm{DCM}$, dried over $\mathrm{Na}_{2} \mathrm{SO}_{4}$. Further filtration, concentration and flash column chromatography afforded the compound S1.

Following the literature procedure ${ }^{14}$, to a solution of S1 (1.0 equiv) in dry THF (0.2 M) was added TMSCl (1.2 equiv.) and the reaction mixture was stirred for $15 \mathrm{~min}$. Then, lithium aluminum hydride (2.0-4.0 equiv) was added in 5 portions. The reaction mixture was then refluxed overnight. After completion, the reaction was quenched by adding water dropwise, $1 \mathrm{~N} \mathrm{NaOH}$ solution, and ethyl acetate. After stirring for $30 \mathrm{~min}$, the reaction mixture was extracted with ethyl acetate, and the organic layer was dried with $\mathrm{Na}_{2} \mathrm{SO}_{4}$. Finally, the solvent was evaporated under vacuum, and the residue was purified by flash chromatography to afford the compound S2.

To a mixture of $\mathbf{S 2}$ (1.0 equiv) and anhydrous $\mathrm{NaHCO}_{3}(2.0$ equiv) in $\mathrm{DMF}(0.25 \mathrm{M})$ was added (E)-1-bromohex-2-ene (1.1 equiv) dropwise at $70{ }^{\circ} \mathrm{C}$ in an oil bath and then stirred for $2 \mathrm{~h}$. After cooled to room temperature, the reaction mixture was extracted with petroleum ether, dried with $\mathrm{Na}_{2} \mathrm{SO}_{4}$, filtered, and concentrated. The residue was purified by column chromatography to give the corresponding allylic amine.

\section{(E)-N,N-Dibenzylhex-2-en-1-amine (1a)}

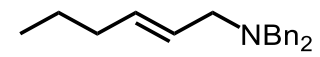

Compound 1a was prepared as a colorless oil in $48 \%$ yield (4.04 g, eluent: petroleum ether/EtOAc $=100 / 1$ to 20/1) from dibenzylamine $(8.4 \mathrm{ml}, 45 \mathrm{mmol})$, trans-2-hexen-1-ol (3.7 $\mathrm{ml}, 30 \mathrm{mmol})$, NBS $(5.34 \mathrm{~g}, 30 \mathrm{mmol})$, and $\mathrm{PPh}_{3}(7.87 \mathrm{~g}, 30 \mathrm{mmol})$ following the above general procedure A.

$\mathbf{R}_{f}=0.67$ (petroleum ether $/$ EtOAc $=15 / 1$ )

${ }^{1} \mathrm{H}$ NMR (400 MHz, $\left.\mathrm{CDCl}_{3}\right) \delta 7.35$ - $7.30(\mathrm{~m}, 4 \mathrm{H}), 7.29-7.22(\mathrm{~m}, 4 \mathrm{H}), 7.21-7.15(\mathrm{~m}, 2 \mathrm{H}), 5.58$ $-5.43(\mathrm{~m}, 2 \mathrm{H}), 3.52(\mathrm{~s}, 4 \mathrm{H}), 2.97(\mathrm{~d}, J=5.7 \mathrm{~Hz}, 2 \mathrm{H}), 2.06-1.88(\mathrm{~m}, 2 \mathrm{H}), 1.44-1.26(\mathrm{~m}, 2 \mathrm{H})$, $0.85(\mathrm{t}, J=7.4 \mathrm{~Hz}, 3 \mathrm{H})$.

${ }^{13} \mathrm{C}$ NMR (100 MHz, $\mathrm{CDCl}_{3}$ ) $\delta$ 140.0, 134.1, 128.9, 128.3, 127.4, 126.9, 57.8, 55.7, 34.7, 22.7, 13.9. 
The ${ }^{1} \mathrm{H}$ and ${ }^{13} \mathrm{C}$ NMR spectroscopic data match the reported characterization data. ${ }^{15}$

Synthesis of 1a'

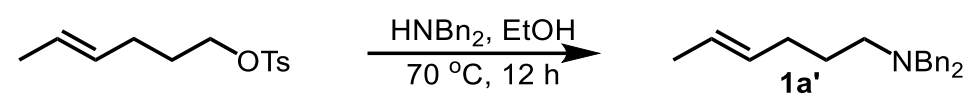

According to a modified literature procedure ${ }^{16}$, (E)-hex-4-en-1-yl 4methylbenzenesulfonate $(0.56 \mathrm{~g}, 2.20 \mathrm{mmol})$ was added to a solution of dibenzyl amine $(0.49$ $\mathrm{mL}, 2.6 \mathrm{mmol})$ in EtOH $(13 \mathrm{~mL})$ and stirred at $70{ }^{\circ} \mathrm{C}$ in an oil bath for $12 \mathrm{~h}$. Upon completion, the reaction mixture was cooled to room temperature and partitioned with $10 \mathrm{~mL}$ of $1 \mathrm{M} \mathrm{KOH}$ and $30 \mathrm{~mL}$ of $\mathrm{CH}_{2} \mathrm{Cl}_{2}$. The organic layer was collected and the aqueous layer was extracted with $\mathrm{CH}_{2} \mathrm{Cl}_{2}(2 \times 15 \mathrm{~mL})$. The combined layers were dried over $\mathrm{Na}_{2} \mathrm{SO}_{4}$ and the solvent was removed to afford a light yellow oil. The crude mixture was purified by silica gel chromatography to afford compound $\mathbf{1 \mathbf { a } ^ { \prime }}$ as a colorless oil in $40 \%$ yield $(0.24 \mathrm{~g}$, eluent: petroleum ether $/ \mathrm{EtOAc}=80 / 1)$.

$\mathbf{R}_{f}=0.60$ (petroleum ether $/$ EtOAc $=20 / 1$ )

${ }^{1} \mathrm{H}$ NMR $\left(400 \mathrm{MHz}, \mathrm{CDCl}_{3}\right)$ \& $7.45-7.40(\mathrm{~m}, 4 \mathrm{H}), 7.40-7.32(\mathrm{~m}, 4 \mathrm{H}), 7.30-7.25(\mathrm{~m}, 2 \mathrm{H}), 5.55$ $-5.47(\mathrm{~m}, 1 \mathrm{H}), 5.44-5.35(\mathrm{~m}, 1 \mathrm{H}), 3.63(\mathrm{~s}, 4 \mathrm{H}), 2.56-2.49(\mathrm{~m}, 2 \mathrm{H}), 2.31-2.23(\mathrm{~m}, 2 \mathrm{H}), 2.09-$ $2.00(\mathrm{~m}, 2 \mathrm{H}), 1.01(\mathrm{t}, J=7.4 \mathrm{~Hz}, 3 \mathrm{H})$.

${ }^{13} \mathrm{C}$ NMR (100 MHz, $\left.\mathrm{CDCl}_{3}\right) \delta$ 140.1, 133.1, 128.9, 128.2, 127.3, 126.9, 58.28, 53.6, 30.5, 25.7, 14.0.

HRMS (ESI-TOF) calcd for $\mathrm{C}_{20} \mathrm{H}_{26} \mathrm{~N}^{+}[\mathrm{M}+\mathrm{H}]^{+}: 280.2060$, found: 280.2069 .

IR (neat, cm$^{-1}$ ): 2930, 2790, 1684, 1507, 965, 743, 697.

(E)-N,N-Dibenzylbut-2-en-1-amine (1b)

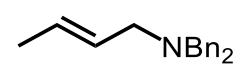

Compound $1 \mathrm{~b}$ was prepared as a colorless oil in $80 \%$ yield $(1.00 \mathrm{~g}$, eluent: petroleum ether $/$ EtOAc $=100 / 1$ to $20 / 1)$ from dibenzylamine $(1.4 \mathrm{ml}, 7.5 \mathrm{mmol})$, crotonyl alcohol $(0.4 \mathrm{ml}$, $5 \mathrm{mmol})$, NBS (0.89 g, $5 \mathrm{mmol})$, and $\mathrm{PPh}_{3}(1.31 \mathrm{~g}, 5 \mathrm{mmol})$ following the above general procedure A.

$\mathbf{R}_{f}=0.59$ (petroleum ether $/ \mathrm{EtOAc}=20 / 1$ )

${ }^{1} \mathrm{H}$ NMR $\left(400 \mathrm{MHz}, \mathrm{CDCl}_{3}\right) \delta 7.45-7.40(\mathrm{~m}, 4 \mathrm{H}), 7.39-7.32(\mathrm{~m}, 4 \mathrm{H}), 7.30-7.25(\mathrm{~m}, 2 \mathrm{H}), 5.72$ $-5.53(\mathrm{~m}, 2 \mathrm{H}), 3.61(\mathrm{~s}, 4 \mathrm{H}), 3.13-2.96(\mathrm{~m}, 2 \mathrm{H}), 1.74(\mathrm{~m}, 3 \mathrm{H})$.

${ }^{13} \mathrm{C}$ NMR (100 MHz, $\left.\mathrm{CDCl}_{3}\right) \delta$ \& 140.0, 129.0, 128.9, 128.6, 126.89, 126.85, 57.8, 55.6, 18.0.

The ${ }^{1} \mathrm{H}$ and ${ }^{13} \mathrm{C}$ NMR spectroscopic data match the reported characterization data. ${ }^{17}$ 
(E)-N,N-Dibenzylundec-2-en-1-amine (1c)

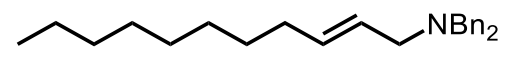

Compound 1c was prepared as a colorless oil in 55\% yield (1.94 g, eluent: petroleum ether/EtOAc $=100 / 1$ to 20/1) from dibenzylamine $(1.4 \mathrm{ml}, 7.5 \mathrm{mmol}),(E)$-undec-2-en-1-ol $(0.4$ $\mathrm{ml}, 5 \mathrm{mmol})$, NBS $(0.89 \mathrm{~g}, 5 \mathrm{mmol})$, and PPh $3(1.31 \mathrm{~g}, 5 \mathrm{mmol})$ following the above general procedure A.

$\mathbf{R}_{f}=0.55$ (petroleum ether $/$ EtOAc $=20 / 1$ )

${ }^{1}$ H NMR (400 MHz, $\left.\mathbf{C D C l}_{3}\right) \delta 7.47-7.43(\mathrm{~m}, 4 \mathrm{H}), 7.38$ (t, J = $\left.7.4 \mathrm{~Hz}, 4 \mathrm{H}\right), 7.30$ (t, J = 7.2 Hz, $2 \mathrm{H}), 5.72-5.54(\mathrm{~m}, 2 \mathrm{H}), 3.64(\mathrm{~s}, 4 \mathrm{H}), 3.09(\mathrm{~d}, J=5.8 \mathrm{~Hz}, 2 \mathrm{H}), 2.10(\mathrm{q}, J=6.8 \mathrm{~Hz}, 2 \mathrm{H}), 1.42-1.31$ $(\mathrm{m}, 12 \mathrm{H}), 0.95(\mathrm{t}, J=6.5 \mathrm{~Hz}, 3 \mathrm{H})$.

${ }^{13} \mathrm{C}$ NMR (100 MHz, $\left.\mathrm{CDCl}_{3}\right) \delta$ 140.0, 134.4, 128.9, 128.3, 127.2, 126.8, 57.8, 55.7, 32.6, 32.0, 29.6, 29.6, 29.5, 29.3, 22.8, 14.3.

The ${ }^{1} \mathrm{H}$ and ${ }^{13} \mathrm{C}$ NMR spectroscopic data match the reported characterization data. ${ }^{18}$

(E)-N-Benzyl-N-methylhex-2-en-1-amine (1d)<smiles>CCC/C=C/CN(C)Cc1ccccc1</smiles>

Compound 1d was prepared as a colorless oil in $60 \%$ yield $(0.61 \mathrm{~g}$, eluent: petroleum ether/EtOAc $=20 / 1)$ from $N$-Methylbenzylamine $(0.7 \mathrm{ml}, 5.00 \mathrm{mmol})$, $(E)$-1-bromohex-2-ene $(0.7 \mathrm{ml}, 5.5 \mathrm{mmol})$, and $\mathrm{NaH}(60 \%, 0.13 \mathrm{~g}, 5.5 \mathrm{mmol})$ following the above general procedure B.

$\mathbf{R}_{f}=0.54$ (petroleum ether $/ \mathrm{EtOAc}=5 / 1$ )

${ }^{1}$ H NMR (400 MHz, $\mathrm{CDCl}_{3}$ ) $\delta 7.39$ - 7.33 (m, 4H), 7.32 - $7.26(\mathrm{~m}, 1 \mathrm{H}), 5.68$ - 5.52 (m, 2H), 3.52 $(\mathrm{s}, 2 \mathrm{H}), 3.02(\mathrm{~d}, J=6.0 \mathrm{~Hz}, 2 \mathrm{H}), 2.22(\mathrm{~s}, 3 \mathrm{H}), 2.07(\mathrm{q}, J=6.9 \mathrm{~Hz}, 2 \mathrm{H}), 1.49-1.41(\mathrm{~m}, 2 \mathrm{H}), 0.95(\mathrm{t}$, $J=7.3 \mathrm{~Hz}, 3 \mathrm{H})$.

${ }^{13} \mathrm{C}$ NMR (100 MHz, $\left.\mathrm{CDCl}_{3}\right) \delta$ 139.1, 134.2, 129.2, 128.2, 127.2, 126.9, 61.6, 59.8, 42.0, 34.5, 22.5, 13.7.

The ${ }^{1} \mathrm{H}$ and ${ }^{13} \mathrm{C}$ NMR spectroscopic data match the reported characterization data. ${ }^{19}$

(E)-N,N-Dibutylhex-2-en-1-amine (1e)<smiles>CCC/C=C/CN(C)C(=O)c1ccccc1</smiles> 
Compound 1e was prepared as a colorless oil in 28\% yield from dibutylamine $(10.3 \mathrm{ml}, 60$ mmol), (E)-1-bromohex-2-ene (1.6 ml, $12 \mathrm{mmol})$, and $\mathrm{NaH}(60 \%, 0.96 \mathrm{~g}, 24 \mathrm{mmol})$ following the above general procedure $B$.

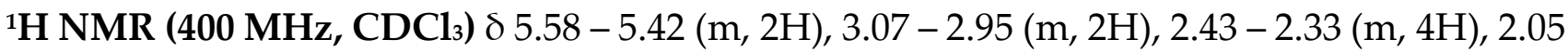
- $1.96(\mathrm{~m}, 2 \mathrm{H}), 1.46-1.35(\mathrm{~m}, 6 \mathrm{H}), 1.32-1.23(\mathrm{~m}, 4 \mathrm{H}), 0.93-0.85(\mathrm{~m}, 9 \mathrm{H})$.

${ }^{13} \mathrm{C}$ NMR (100 MHz, $\left.\mathrm{CDCl}_{3}\right) \delta$ 133.6, 127.5, 56.5, 53.5, 34.7, 29.2, 22.6, 20.9, 14.2, 13.8.

HRMS (ESI-TOF) calcd for $\mathrm{C}_{14} \mathrm{H}_{30} \mathrm{~N}^{+}[\mathrm{M}+\mathrm{H}]^{+}:$212.2373, found: 212.2381.

IR (neat, cm-1): 2956, 2871, 2359, 1684, 1653, 1558, 1540, 1507, 1457, 970.

\section{Methyl (E)-1-(hex-2-en-1-yl)piperidine-4-carboxylate (1f)}

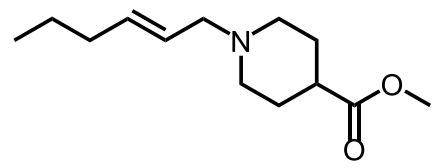

Compound 1 f was prepared as a colorless oil in $11 \%$ yield $(0.12 \mathrm{~g}$, eluent: $\mathrm{DCM} / \mathrm{MeOH}=40 / 1)$ from $(E)$-1-bromohex-2-ene $(0.7 \mathrm{ml}, 5.00 \mathrm{mmol})$, methyl piperidine-4-carboxylate $(0.72 \mathrm{~g}, 5.00$ $\mathrm{mmol})$, and $\mathrm{K}_{2} \mathrm{CO}_{3}(2.07 \mathrm{~g}, 15.00 \mathrm{mmol})$, following the above general procedure $\mathrm{D}$.

$\mathbf{R}_{f}=0.35(\mathrm{DCM} / \mathrm{MeOH}=10 / 1)$

${ }^{1} \mathrm{H}$ NMR (400 MHz, $\mathrm{CDCl}_{3}$ ) $\delta 5.61$ - 5.52 (m, 1H), 5.51 - 5.43 (m, 1H), 3.67 (s, 3H), 2.98 - 2.84 (m, 4H), $2.33-2.22(\mathrm{~m}, 1 \mathrm{H}), 2.03-1.87(\mathrm{~m}, 6 \mathrm{H}), 1.81-1.71(\mathrm{~m}, 2 \mathrm{H}), 1.43-1.34(\mathrm{~m}, 2 \mathrm{H}), 0.89$ $(\mathrm{t}, J=7.4 \mathrm{~Hz}, 3 \mathrm{H})$.

${ }^{13} \mathrm{C}$ NMR (101 MHz, $\left.\mathrm{CDCl}_{3}\right) \delta$ 175.7, 134.6, 126.6, 61.3, 52.9, 51.8, 41.2, 34.6, 28.4, 22.5, 13.8.

HRMS (ESI-TOF) calcd for $\mathrm{C}_{13} \mathrm{H}_{24} \mathrm{NO}_{2}{ }^{+}[\mathrm{M}+\mathrm{H}]^{+}:$226.1802, found: 226.1812 .

IR (neat, cm-1): 2955, 2830, 2361, 1718, 1684, 1653, 1559, 1540, 1507, 1457.

\section{Methyl (R,E)-1-(hex-2-en-1-yl)piperidine-3-carboxylate (1g)}

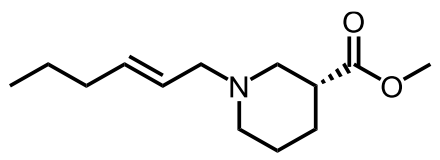

Compound $1 \mathrm{~g}$ was prepared as a colorless oil in 75\% yield ( $0.66 \mathrm{~g}$, eluent: DCM/MeOH=40/1) from $(E)$-1-bromohex-2-ene $(0.5 \mathrm{ml}, 3.90 \mathrm{mmol})$, methyl $(R)$-piperidine-3-carboxylate $(0.56 \mathrm{~g}$, $3.90 \mathrm{mmol})$, and $\mathrm{K}_{2} \mathrm{CO}_{3}(1.62 \mathrm{~g}, 11.70 \mathrm{mmol})$, following the above general procedure $\mathrm{D}$.

$\mathbf{R}_{f}=0.45(\mathrm{DCM} / \mathrm{MeOH}=10 / 1)$

${ }^{1} \mathrm{H}$ NMR (400 MHz, $\left.\mathrm{CDCl}_{3}\right) \delta 5.63-5.52(\mathrm{~m}, 1 \mathrm{H}), 5.52-5.40(\mathrm{~m}, 1 \mathrm{H}), 3.65(\mathrm{~s}, 3 \mathrm{H}), 3.04-2.96$ $(\mathrm{m}, 1 \mathrm{H}), 2.93(\mathrm{~d}, J=6.6,1.1 \mathrm{~Hz}, 2 \mathrm{H}), 2.85-2.73(\mathrm{~m}, 1 \mathrm{H}), 2.66-2.49(\mathrm{~m}, 1 \mathrm{H}), 2.09-1.87(\mathrm{~m}$, 
$5 \mathrm{H}), 1.78-1.62(\mathrm{~m}, 1 \mathrm{H}), 1.62-1.51(\mathrm{~m}, 1 \mathrm{H}), 1.46-1.34(\mathrm{~m}, 3 \mathrm{H}), 0.88(\mathrm{t}, J=7.4 \mathrm{~Hz}, 3 \mathrm{H})$.

${ }^{13} \mathrm{C}$ NMR (100 MHz, $\left.\mathrm{CDCl}_{3}\right) \delta$ 174.8, 134.6, 126.4, 61.3, 55.3, 53.6, 51.7, 42.0, 34.6, 27.2, 24.8, $22.5,13.8$.

HRMS (ESI-TOF) calcd for $\mathrm{C}_{13} \mathrm{H}_{24} \mathrm{NO}_{2}{ }^{+}[\mathrm{M}+\mathrm{H}]^{+}:$: 226.1802, found: 226.1813.

IR (neat, cm$^{-1}$ ): 2954, 2870, 2802, 1735, 1559, 1507, 1457, 1152, 973.

Methyl (E)-hex-2-en-1-yl-L-prolinate (1h)

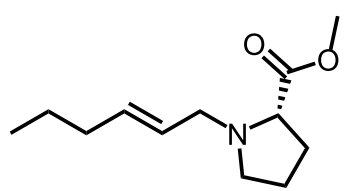

Compound $1 \mathrm{~h}$ was prepared as a colorless oil in 54\% yield (0.78 $\mathrm{g}$, eluent: petroleum ether/EtOAc $=8 / 1)$ from methyl $D$-prolinate $(0.89 \mathrm{~g}, 6.90 \mathrm{mmol}),(E)$-1-bromohex-2-ene $(0.9$ $\mathrm{ml}, 6.90 \mathrm{mmol})$, and $\mathrm{K}_{2} \mathrm{CO}_{3}(2.85 \mathrm{~g}, 21 \mathrm{mmol})$ following the above general procedure $\mathrm{D}$.

$\mathbf{R}_{f}=0.29$ (petroleum ether $/$ EtOAc $=2 / 1$ )

${ }^{1}$ H NMR (400 MHz, $\mathrm{CDCl}_{3}$ ) $\delta 5.64-5.43(\mathrm{~m}, 2 \mathrm{H}), 3.68(\mathrm{~s}, 3 \mathrm{H}), 3.20$ - $3.03(\mathrm{~m}, 4 \mathrm{H}), 2.36-2.28$ $(\mathrm{m}, 1 \mathrm{H}), 2.16-2.07(\mathrm{~m}, 1 \mathrm{H}), 1.98-1.87(\mathrm{~m}, 4 \mathrm{H}), 1.82-1.71(\mathrm{~m}, 1 \mathrm{H}), 1.39-1.30(\mathrm{~m}, 2 \mathrm{H}), 0.86$ $(\mathrm{t}, J=7.3 \mathrm{~Hz}, 3 \mathrm{H})$.

${ }^{13} \mathrm{C}$ NMR (100 MHz, $\left.\mathrm{CDCl}_{3}\right) \delta$ 174.8, 134.3, 126.7, 65.3, 57.0, 53.6, 51.9, 34.5, 29.66, 23.2, 22.4, 13.8.

HRMS (ESI-TOF) calcd for $\mathrm{C}_{12} \mathrm{H}_{22} \mathrm{NO}_{2}{ }^{+}[\mathrm{M}+\mathrm{H}]^{+}:$212.1645, found: 212.1652 .

IR (neat, cm-1): 2957, 2871, 1734, 1558, 1457, 1169, 970.

(E)-8-(Hex-2-en-1-yl)-1,4-dioxa-8-azaspiro[4.5]decane (1j)

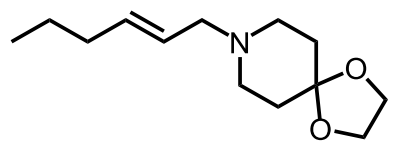

Compound $\mathbf{1} \mathbf{j}$ was prepared as a colorless oil in $60 \%$ yield $(0.34 \mathrm{~g}$, eluent: $\mathrm{DCM} / \mathrm{MeOH}=20 / 1)$ from 1,4-dioxa-8-azaspiro[4.5]decane $(0.3 \mathrm{ml}, 2.50 \mathrm{mmol}),(E)$-1-bromohex-2-ene (0.7 $\mathrm{ml}, 5.00$ $\mathrm{mmol})$, and saturated aqueous $\mathrm{K}_{2} \mathrm{CO}_{3}(6.5 \mathrm{ml})$ following the above general procedure $\mathrm{C}$.

$\mathbf{R}_{f}=0.65(\mathrm{DCM} / \mathrm{MeOH}=10 / 1)$

${ }^{1}$ H NMR (400 MHz, $\left.\mathrm{CDCl}_{3}\right) \delta 5.61-5.44(\mathrm{~m}, 2 \mathrm{H}), 3.94(\mathrm{~s}, 4 \mathrm{H}), 2.96(\mathrm{~d}, J=7.1 \mathrm{~Hz}, 2 \mathrm{H}), 2.51$ (s, $4 \mathrm{H}), 2.04-1.96(\mathrm{~m}, 2 \mathrm{H}), 1.75(\mathrm{t}, J=5.7 \mathrm{~Hz}, 4 \mathrm{H}), 1.43-1.32(\mathrm{~m}, 2 \mathrm{H}), 0.88(\mathrm{t}, J=7.3 \mathrm{~Hz}, 3 \mathrm{H})$.

${ }^{13} \mathrm{C}$ NMR (100 MHz, $\left.\mathrm{CDCl}_{3}\right) \delta$ 134. 7, 126.9, 107.5, 64.4, 60.9, 51.4, 35.1, 34.7, 22.6, 14.0.

HRMS (ESI-TOF) calcd for $\mathrm{C}_{13} \mathrm{H}_{24} \mathrm{NO}_{2}{ }^{+}[\mathrm{M}+\mathrm{H}]^{+}$: 226.1802, found: 226.1810 . 
IR (neat, cm-1): 2956, 2927, 2872, 2810, 1559, 1507, 1363, 1150, 965, 946, 912.

(E)-N-Ethyl-N-(hex-2-en-1-yl)-3-methylaniline (10)

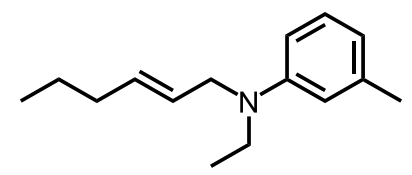

Compound 10 was prepared as a colorless oil in $92 \%$ yield $(0.57 \mathrm{~g}$, eluent: petroleum ether/EtOAc = 50/1) from $N$-ethyl-3-methylaniline $(0.4 \mathrm{ml}, 2.50 \mathrm{mmol}),(E)$-1-bromohex-2-ene $(0.7 \mathrm{ml}, 5.00 \mathrm{mmol})$, and saturated aqueous $\mathrm{K}_{2} \mathrm{CO}_{3}(6.5 \mathrm{ml})$ following the above general procedure $C$.

$\mathbf{R}_{f}=0.85$ (petroleum ether $/$ EtOAc $=10 / 1$ )

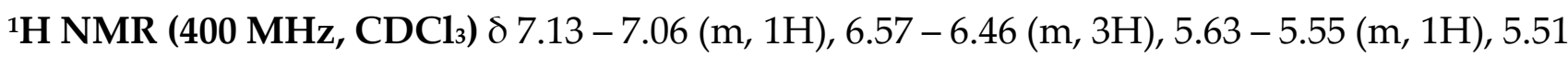
$-5.42(\mathrm{~m}, 1 \mathrm{H}), 3.83(\mathrm{~d}, J=5.0 \mathrm{~Hz}, 2 \mathrm{H}), 3.36(\mathrm{q}, J=7.1 \mathrm{~Hz}, 2 \mathrm{H}), 2.30(\mathrm{~s}, 3 \mathrm{H}), 2.04-1.98(\mathrm{~m}, 2 \mathrm{H})$, $1.42-1.34(\mathrm{~m}, 2 \mathrm{H}), 1.15(\mathrm{t}, J=7.1 \mathrm{~Hz}, 3 \mathrm{H}), 0.88(\mathrm{t}, J=7.4 \mathrm{~Hz}, 3 \mathrm{H})$.

${ }^{13} \mathrm{C}$ NMR (100 MHz, $\left.\mathrm{CDCl}_{3}\right) \delta$ 148.6, 138.9, 132.4, 129.1, 126.3, 116.7, 113.0, 109.5, 52.0, 44.5, $34.5,22.6,22.1,13.8,12.4$.

HRMS (ESI-TOF) calcd for $\mathrm{C}_{15} \mathrm{H}_{24} \mathrm{~N}^{+}[\mathrm{M}+\mathrm{H}]^{+}: 218.1903$, found: 218.1906.

IR (neat, cm-1): 2962, 2926, 2871, 2370, 1600, 1507, 1496, 1176, 969, 762, 691.

(E)-N-Butyl-N-(hex-2-en-1-yl)aniline (1p)

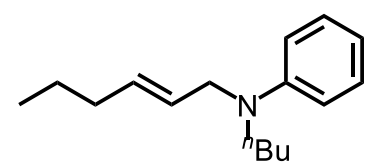

Compound $1 \mathrm{p}$ was prepared as a colorless oil in $95 \%$ yield $(0.58 \mathrm{~g}$, eluent: petroleum ether/EtOAc $=50 / 1)$ from $N$-butylaniline $(0.4 \mathrm{ml}, 2.50 \mathrm{mmol}),(E)$-1-bromohex-2-ene $(0.7 \mathrm{ml}$, $5.00 \mathrm{mmol})$, and saturated aqueous $\mathrm{K}_{2} \mathrm{CO}_{3}(6.5 \mathrm{ml})$ following the above general procedure $\mathrm{C}$. $\mathbf{R}_{f}=0.93$ (petroleum ether $/$ EtOAc $=10 / 1$ )

${ }^{1}$ H NMR (400 MHz, $\mathrm{CDCl}_{3}$ ) $\delta 7.25$ - 7.17 (m, 2H), 6.73 - $6.62(\mathrm{~m}, 3 \mathrm{H}), 5.63-5.54$ (m, 1H), 5.50 - $5.42(\mathrm{~m}, 1 \mathrm{H}), 3.86(\mathrm{~d}, J=5.1 \mathrm{~Hz}, 2 \mathrm{H}), 3.31-3.24(\mathrm{~m}, 2 \mathrm{H}), 2.05-1.98(\mathrm{~m}, 2 \mathrm{H}), 1.62-1.54(\mathrm{~m}$, 2H), $1.43-1.32(\mathrm{~m}, 4 \mathrm{H}), 0.96(\mathrm{t}, J=7.3 \mathrm{~Hz}, 3 \mathrm{H}), 0.89(\mathrm{t}, J=7.3 \mathrm{~Hz}, 3 \mathrm{H})$.

${ }^{13} \mathrm{C}$ NMR (100 MHz, $\left.\mathrm{CDCl}_{3}\right) \delta$ 148.7, 132.5, 129.2, 126.0, 115.6, 112.1, 52.6, 50.3, 34.5, 29.6, 22.6, 20.5, 14.2, 13.8 .

HRMS (ESI-TOF) calcd for $\mathrm{C}_{16} \mathrm{H}_{26} \mathrm{~N}^{+}[\mathrm{M}+\mathrm{H}]^{+}: 232.2026$, found: 232.2064 .

IR (neat, cm ${ }^{-1}$ ): 2956, 2926, 2871, 2322, 1596, 1506, 1457, 967, 743, 690. 
(E)-N-(Hex-2-en-1-yl)-N-isopropylaniline (1q)<smiles>CCC/C=C/CN(CCC)c1ccccc1</smiles>

Compound $1 \mathbf{~ q ~ w a s ~ p r e p a r e d ~ a s ~ a ~ c o l o r l e s s ~ o i l ~ i n ~} 73 \%$ yield $(0.40 \mathrm{~g}$, eluent: petroleum ether/EtOAc $=50 / 1)$ from $N$-isopropylaniline $(0.4 \mathrm{ml}, 2.50 \mathrm{mmol}),(E)$-1-bromohex-2-ene $(0.7$ $\mathrm{ml}, 5.00 \mathrm{mmol})$, and saturated aqueous $\mathrm{K}_{2} \mathrm{CO}_{3}(6.5 \mathrm{ml})$ following the above general procedure C.

$\mathbf{R}_{f}=0.59$ (petroleum ether $/$ EtOAc $=10 / 1$ )

${ }^{1} \mathrm{H}$ NMR (400 MHz, $\left.\mathrm{CDCl}_{3}\right) \delta 7.28-7.21(\mathrm{~m}, 2 \mathrm{H}), 6.84-6.78(\mathrm{~m}, 2 \mathrm{H}), 6.77-6.65(\mathrm{~m}, 1 \mathrm{H}), 5.74$ - $5.60(\mathrm{~m}, 1 \mathrm{H}), 5.60-5.45(\mathrm{~m}, 1 \mathrm{H}), 4.20-4.10(\mathrm{~m}, 1 \mathrm{H}), 3.80(\mathrm{~d}, J=3.1 \mathrm{~Hz}, 2 \mathrm{H}), 2.08-2.00(\mathrm{~m}$, $2 \mathrm{H}), 1.46-1.38(\mathrm{~m}, 2 \mathrm{H}), 1.23(\mathrm{~d}, J=6.7 \mathrm{~Hz}, 6 \mathrm{H}), 0.92(\mathrm{t}, J=7.4 \mathrm{~Hz}, 3 \mathrm{H})$.

${ }^{13} \mathrm{C}$ NMR (100 MHz, $\left.\mathrm{CDCl}_{3}\right) \delta$ 149.3, 131.5, 129.1, 128.5, 115.9, 113.0, 48.0, 46.2, 34.5, 22.6, 20.1, 13.8.

HRMS (ESI-TOF) calcd for $\mathrm{C}_{15} \mathrm{H}_{24} \mathrm{~N}^{+}[\mathrm{M}+\mathrm{H}]^{+}:$: 218.1903, found: 218.1911.

IR (neat, cm-1): 2962, 2321, 2928, 2871, 1596, 1505, 1186, 970, 744, 690.

(E)-N-Benzyl-N-(hex-2-en-1-yl)aniline (1r)<smiles>CCC/C=C/CN(Cc1ccccc1)c1ccccc1</smiles>

Compound 1r was prepared as a colorless oil in 91\% yield $(0.60 \mathrm{~g}$, eluent: petroleum ether/EtOAc $=100 / 1)$ from $N$-benzylaniline $(0.4 \mathrm{ml}, 2.50 \mathrm{mmol}),(E)$-1-bromohex-2-ene $(0.5 \mathrm{ml}$, $3.75 \mathrm{mmol})$, and $\mathrm{NaHCO}_{3}(0.31 \mathrm{~g}, 4.75 \mathrm{mmol})$ following the above general procedure $\mathrm{E}$.

$\mathbf{R}_{f}=0.91$ (petroleum ether $/$ EtOAc $=10 / 1$ )

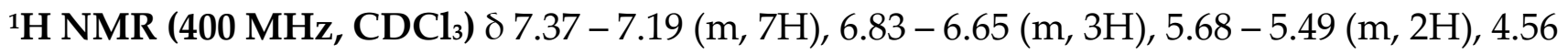
(s, 2H), $3.99(\mathrm{~d}, J=5.6 \mathrm{~Hz}, 2 \mathrm{H}), 2.11-2.00(\mathrm{~m}, 2 \mathrm{H}), 1.47-1.36(\mathrm{~m}, 2 \mathrm{H}), 0.92(\mathrm{t}, J=7.4 \mathrm{~Hz}, 3 \mathrm{H})$. ${ }^{13} \mathrm{C}$ NMR (100 MHz, $\left.\mathrm{CDCl}_{3}\right) \delta$ 149.2, 139.3, 133.2, 129.2, 128.7, 126.9, 126.7, 125.3, 116.4, 112.5, $53.8,52.5,34.5,22.6,13.8$.

HRMS (ESI-TOF) calcd for $\mathrm{C}_{19} \mathrm{H}_{24} \mathrm{~N}^{+}[\mathrm{M}+\mathrm{H}]^{+}$: 266.1903, found: 266.1911.

IR (neat, cm$^{-1}$ ): 2957, 2926, 9870, 2320, 1597, 1506, 745, 727, 691.

(E)-N-(Hex-2-en-1-yl)-N,4-dimethylaniline (1s)

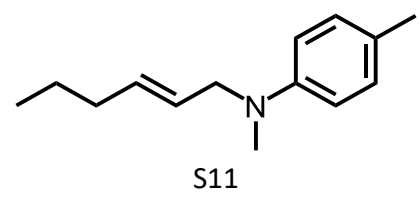


Compound 1s was prepared as a colorless oil in $43 \%$ yield $(0.22 \mathrm{~g}$, eluent: petroleum ether/EtOAc $=100 / 1)$ from $N$,4-dimethylaniline $(0.3 \mathrm{ml}, 2.50 \mathrm{mmol})$, $(E)$-1-bromohex-2-ene $(0.7 \mathrm{ml}, 5.00 \mathrm{mmol})$, and saturated aqueous $\mathrm{K}_{2} \mathrm{CO}_{3}(6.5 \mathrm{ml})$ following the above general procedure C.

$\mathbf{R}_{f}=0.87$ (petroleum ether $/$ EtOAc $=15 / 1$ )

${ }^{1} \mathrm{H}$ NMR (400 MHz, $\left.\mathrm{CDCl}_{3}\right) \delta 7.17-6.90(\mathrm{~m}, 2 \mathrm{H}), 6.83-6.56(\mathrm{~m}, 2 \mathrm{H}), 5.66-5.54(\mathrm{~m}, 1 \mathrm{H}), 5.52$ $-5.39(\mathrm{~m}, 1 \mathrm{H}), 3.82(\mathrm{~d}, J=5.5 \mathrm{~Hz}, 2 \mathrm{H}), 2.86(\mathrm{~s}, 3 \mathrm{H}), 2.26(\mathrm{~s}, 3 \mathrm{H}), 2.04-1.95(\mathrm{~m}, 2 \mathrm{H}), 1.43-1.33$ $(\mathrm{m}, 2 \mathrm{H}), 0.88(\mathrm{t}, J=7.4 \mathrm{~Hz}, 3 \mathrm{H})$.

${ }^{13}$ C NMR (100 MHz, $\mathrm{CDCl}_{3}$ ) $\delta 147.9,133.1,129.7,125.8,125.6,113.3,55.2,38.1,34.5,22.6,20.4$, 13.8.

HRMS (ESI-TOF) calcd for $\mathrm{C}_{14} \mathrm{H}_{22} \mathrm{~N}^{+}[\mathrm{M}+\mathrm{H}]^{+}:$204.1747, found: 204.1756.

IR (neat, cm$^{-1}$ ): 2956, 2322, 1520, 1362, 970, 801.

(E)-N-(Hex-2-en-1-yl)-N,3-dimethylaniline (1t)

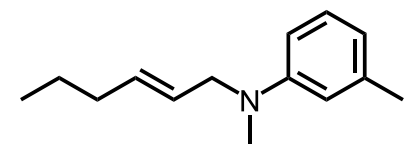

Compound $1 \mathrm{t}$ was prepared as a colorless oil in $68 \%$ yield $(0.35 \mathrm{~g}$, eluent: petroleum ether/EtOAc $=50 / 1)$ from $N, 3$-dimethylaniline $(0.3 \mathrm{ml}, 2.50 \mathrm{mmol}),(E)$-1-bromohex-2-ene $(0.7$ $\mathrm{ml}, 5.00 \mathrm{mmol})$, saturated and aqueous $\mathrm{K}_{2} \mathrm{CO}_{3}(6.5 \mathrm{ml})$ following the above general procedure C.

$\mathbf{R}_{f}=0.67$ (petroleum ether $/$ EtOAc $=10 / 1$ )

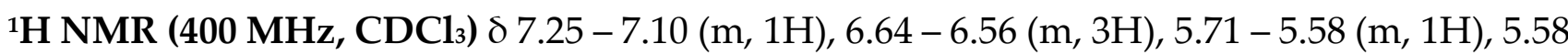
- $5.43(\mathrm{~m}, 1 \mathrm{H}), 3.90(\mathrm{~d}, J=4.9 \mathrm{~Hz}, 2 \mathrm{H}), 2.94(\mathrm{~s}, 3 \mathrm{H}), 2.37(\mathrm{~s}, 3 \mathrm{H}), 2.09-2.01(\mathrm{~m}, 2 \mathrm{H}), 1.48-1.39$ $(\mathrm{m}, 2 \mathrm{H}), 0.94(\mathrm{t}, J=7.4 \mathrm{~Hz}, 3 \mathrm{H})$.

${ }^{13} \mathrm{C}$ NMR (100 MHz, $\left.\mathrm{CDCl}_{3}\right) \delta$ 149.9, 138.8, 133.0, 129.1, 125.6, 117.4, 113.6, 110.0, 54.8, 37.8, $34.5,22.6,22.1,13.7$.

HRMS (ESI-TOF) calcd for $\mathrm{C}_{14} \mathrm{H}_{22} \mathrm{~N}^{+}[\mathrm{M}+\mathrm{H}]^{+}:$204.1747, found: 204.1747.

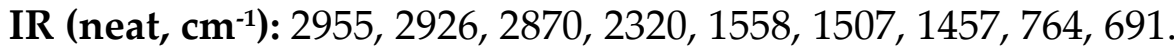

(E)-4-Fluoro-N-(hex-2-en-1-yl)- N-methylaniline (1v)<smiles>CCC/C=C/CN(C)c1ccc(F)cc1</smiles> 
Compound $1 \mathbf{v}$ was prepared as a colorless oil in $57 \%$ yield $(0.30 \mathrm{~g}$, eluent: petroleum ether/EtOAc $=100 / 1)$ from 4-fluoro- $N$-methylaniline $(0.3 \mathrm{ml}, 2.50 \mathrm{mmol}),(E)-1$-bromohex-2ene $(0.7 \mathrm{ml}, 5.00 \mathrm{mmol})$, and saturated aqueous $\mathrm{K}_{2} \mathrm{CO}_{3}(6.5 \mathrm{ml})$ following the above general procedure $C$.

$\mathbf{R}_{f}=0.77$ (petroleum ether/EtOAc $=10 / 1$ )

${ }^{1} \mathrm{H}$ NMR (400 MHz, $\left.\mathrm{CDCl}_{3}\right) \delta 7.00-6.87(\mathrm{~m}, 2 \mathrm{H}), 6.82-6.57(\mathrm{~m}, 2 \mathrm{H}), 5.65-5.53(\mathrm{~m}, 1 \mathrm{H}), 5.50$ - $5.38(\mathrm{~m}, 1 \mathrm{H}), 3.80(\mathrm{~d}, J=5.2 \mathrm{~Hz}, 2 \mathrm{H}), 2.86(\mathrm{~s}, 3 \mathrm{H}), 2.06-1.94(\mathrm{~m}, 2 \mathrm{H}), 1.43-1.33(\mathrm{~m}, 2 \mathrm{H})$, $0.87(\mathrm{t}, J=7.4 \mathrm{~Hz}, 3 \mathrm{H})$.

${ }^{13} \mathrm{C}$ NMR (100 MHz, $\left.\mathrm{CDCl}_{3}\right) \delta 155.5(\mathrm{~d}, J=234.0 \mathrm{~Hz}), 146.6(\mathrm{~d}, J=2.0 \mathrm{~Hz}), 133.39,125.32,115.5$ $(\mathrm{d}, J=21.0 \mathrm{~Hz}), 114.2(\mathrm{~d}, J=7.0 \mathrm{~Hz}), 55.62,38.45,34.47,22.56,13.74$.

${ }^{19}$ F NMR (377 MHz, $\left.\mathrm{CDCl}_{3}\right) \delta-129.4$.

HRMS (ESI-TOF) calcd for $\mathrm{C}_{13} \mathrm{H}_{19} \mathrm{FN}^{+}[\mathrm{M}+\mathrm{H}]^{+}:$208.1496, found: 208.1499.

IR (neat, cm$^{-1}$ ): 2957, 2930, 2871, 1558, 1507, 1227, 970, 812.

\section{(E)-4-Chloro-N-(hex-2-en-1-yl)-N-methylaniline (1w)}<smiles>CCC/C=C/CN(C)c1ccc(Cl)cc1</smiles>

Compound 1w was prepared as a colorless oil in 59\% yield (0.33 g, eluent: petroleum ether/EtOAc = 100/1) from 4-chloro- $N$-methylaniline $(0.3 \mathrm{ml}, 2.50 \mathrm{mmol}),(E)-1$-bromohex-2ene $(0.7 \mathrm{ml}, 5.00 \mathrm{mmol})$, and saturated aqueous $\mathrm{K}_{2} \mathrm{CO}_{3}(6.5 \mathrm{ml})$ following the above general procedure $C$.

$\mathbf{R}_{f}=0.84$ (petroleum ether $/$ EtOAc $=10 / 1$ )

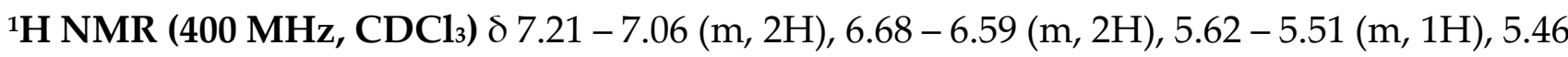
- $5.37(\mathrm{~m}, 1 \mathrm{H}), 3.83(\mathrm{~d}, J=4.6 \mathrm{~Hz}, 2 \mathrm{H}), 2.89(\mathrm{~s}, 3 \mathrm{H}), 2.04-1.96(\mathrm{~m}, 2 \mathrm{H}), 1.43-1.32(\mathrm{~m}, 2 \mathrm{H})$, $0.88(\mathrm{t}, J=7.4 \mathrm{~Hz}, 3 \mathrm{H})$.

${ }^{13} \mathrm{C}$ NMR (100 MHz, $\left.\mathrm{CDCl}_{3}\right) \delta$ 148.3, 133.3, 128.9, 124.9, 121.1, 113.8, 54.8, 38.1, 34.5, 22.5, 13.8. HRMS (ESI-TOF) calcd for $\mathrm{C}_{13} \mathrm{H}_{19} \mathrm{ClN}^{+}[\mathrm{M}+\mathrm{H}]^{+}$: 224.1201, found: 224.1210.

IR (neat, cm-1): 2956, 2927, 2870, 2362, 1596, 1497, 1374, 1198, 969, 806.

\section{Methyl (E)-4-(hex-2-en-1-yl(methyl)amino)benzoate (1x)}<smiles>CCC/C=C/CN(C)c1ccc(C(=O)OC)cc1</smiles> 
Compound 1x was prepared as a colorless oil in 63\% yield (0.48 g, eluent: petroleum ether/EtOAc $=50 / 1)$ from methyl 4-(methylamino)benzoate $(0.52 \mathrm{~g}, 3.10 \mathrm{mmol})$, $(E)-1$ bromohex-2-ene $(0.6 \mathrm{ml}, 4.65 \mathrm{mmol})$, and $\mathrm{NaHCO}_{3}(0.39 \mathrm{~g}, 4.65 \mathrm{mmol})$ following the above general procedure $\mathrm{E}$.

$\mathbf{R}_{f}=0.49$ (petroleum ether $/$ EtOAc $=10 / 1$ )

${ }^{1}$ H NMR (400 MHz, $\left.\mathrm{CDCl}_{3}\right) \delta 7.88(\mathrm{~d}, J=8.9 \mathrm{~Hz}, 2 \mathrm{H}), 6.65(\mathrm{~d}, J=8.9 \mathrm{~Hz}, 2 \mathrm{H}), 5.64-5.49$ (m, 1H), $5.49-5.33(\mathrm{~m}, 1 \mathrm{H}), 3.92(\mathrm{~d}, J=5.3 \mathrm{~Hz}, 2 \mathrm{H}), 3.85(\mathrm{~s}, 3 \mathrm{H}), 2.99(\mathrm{~s}, 3 \mathrm{H}), 2.00$ (q, J = 7.2 Hz, $2 \mathrm{H}), 1.41-1.32(\mathrm{~m}, 2 \mathrm{H}), 0.87(\mathrm{t}, J=7.4 \mathrm{~Hz}, 3 \mathrm{H})$.

${ }^{13} \mathrm{C}$ NMR (100 MHz, $\left.\mathrm{CDCl}_{3}\right) \delta 167.6,152.7,133.4,131.4,124.2,116.9,110.9,54.1,51.6,37.9$, $34.4,22.5,13.7$.

HRMS (ESI-TOF) calcd for $\mathrm{C}_{15} \mathrm{H}_{22} \mathrm{FNO}_{2}{ }^{+}[\mathrm{M}+\mathrm{H}]^{+}:$248.1645, found: 248.1649.

IR (neat, cm-1): 2955, 2930, 2871, 2319, 1701, 1606, 1280, 1182, 1109, 769.

\section{(E)-1-(Hex-2-en-1-yl)-1,2,3,4-tetrahydroquinoline (1y)}

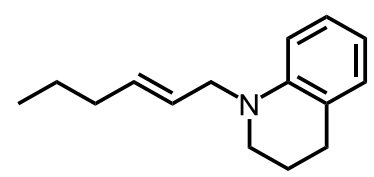

Compound 1y was prepared as a colorless oil in $76 \%$ yield $(0.41 \mathrm{~g}$, eluent: petroleum ether/EtOAc $=50 / 1)$ 1,2,3,4-tetrahydroquinoline $(0.3 \mathrm{ml}, 2.50 \mathrm{mmol}),(E)$-1-bromohex-2-ene $(0.7 \mathrm{ml}, 5.00 \mathrm{mmol})$, and saturated aqueous $\mathrm{K}_{2} \mathrm{CO}_{3}(6.5 \mathrm{ml})$ following the above general procedure $C$.

$\mathbf{R}_{f}=0.71$ (petroleum ether $/$ EtOAc $=10 / 1$ )

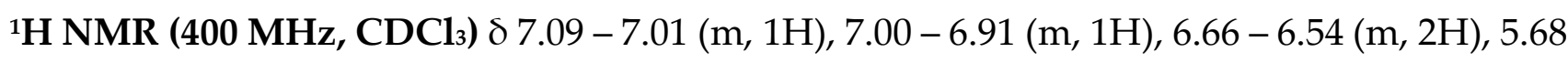
- $5.57(\mathrm{~m}, 1 \mathrm{H}), 5.53-5.44(\mathrm{~m}, 1 \mathrm{H}), 3.83(\mathrm{~d}, J=4.6 \mathrm{~Hz}, 2 \mathrm{H}), 3.27(\mathrm{t}, J=5.7 \mathrm{~Hz}, 2 \mathrm{H}), 2.78(\mathrm{t}, J=$ $6.4 \mathrm{~Hz}, 2 \mathrm{H}), 2.05-1.95(\mathrm{~m}, 4 \mathrm{H}), 1.44-1.36(\mathrm{~m}, 2 \mathrm{H}), 0.91(\mathrm{t}, J=7.4 \mathrm{~Hz}, 3 \mathrm{H})$.

${ }^{13} \mathrm{C}$ NMR (100 MHz, $\left.\mathrm{CDCl}_{3}\right) \delta$ 145.6, 132.7, 129.1, 127.1, 125.2, 122.6, 115.7, 111.2, 53.2, 48.9, $34.5,28.3,22.6,22.5,13.8$.

HRMS (ESI-TOF) calcd for $\mathrm{C}_{15} \mathrm{H}_{22} \mathrm{~N}^{+}[\mathrm{M}+\mathrm{H}]^{+}: 216.1747$, found: 216.1757 .

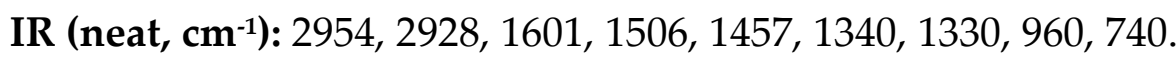

\section{Synthesis of $1 z$}




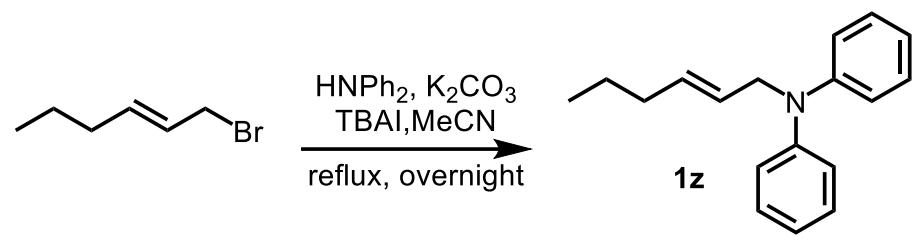

According to a modified literature procedure ${ }^{20}$, a solution of diphenylamine $(0.85 \mathrm{~g}, 5.00$ mmol, 1.0 equiv) in acetonitrile was treated with $\mathrm{K}_{2} \mathrm{CO}_{3}(1.38 \mathrm{~g} 10.00 \mathrm{mmol}, 2.0$ equiv), (E)1-bromohex-2-ene (1.3 mL, $10.00 \mathrm{mmol}, 2.0$ equiv), and TBAI (92 mg, $0.25 \mathrm{mmol}, 5 \mathrm{~mol} \%$ ). The reaction mixture was heated at reflux in an oil bath and was monitored by TLC (petroleum ether). The solution was cooled to room temperature, diluted with $\mathrm{H}_{2} \mathrm{O}$ and extracted with EtOAc. The combined organic layers were dried with $\mathrm{MgSO}_{4}$ and concentrated in vacuo. The residue was purified by chromatography to afford compound $\mathbf{1 z}$ as a colorless oil in $43 \%$ yield ( $0.53 \mathrm{~g}$, eluent: petroleum ether).

$\mathbf{R}_{f}=0.43$ (petroleum ether)

${ }^{1} \mathrm{H}$ NMR (400 MHz, $\left.\mathrm{CDCl}_{3}\right) \delta 7.35-7.24(\mathrm{~m}, 4 \mathrm{H}), 7.09(\mathrm{~d}, J=7.9 \mathrm{~Hz}, 4 \mathrm{H}), 7.00(\mathrm{t}, J=7.3 \mathrm{~Hz}$, 2H), $5.76-5.54(\mathrm{~m}, 2 \mathrm{H}), 4.36(\mathrm{~d}, J=4.3 \mathrm{~Hz}, 2 \mathrm{H}), 2.04(\mathrm{q}, J=7.1 \mathrm{~Hz}, 2 \mathrm{H}), 1.44-1.35(\mathrm{~m}, 2 \mathrm{H})$, $0.89(\mathrm{t}, J=7.4 \mathrm{~Hz}, 3 \mathrm{H})$.

${ }^{13} \mathrm{C}$ NMR (100 MHz, $\left.\mathrm{CDCl}_{3}\right) \delta$ 148.1, 133.1, 129.3, 125.9, 121.2, 121.0, 54.3, 34.5, 22.5, 13.7. HRMS (ESI-TOF) calcd for $\mathrm{C}_{18} \mathrm{H}_{22} \mathrm{~N}^{+}[\mathrm{M}+\mathrm{H}]^{+}$: 252.1747, found: 252.1752.

IR (neat, cm-1): 2956, 2930, 2871, 1589, 1492, 1362, 1223, 968, 745, 690.

\section{Synthesis of 1aa}

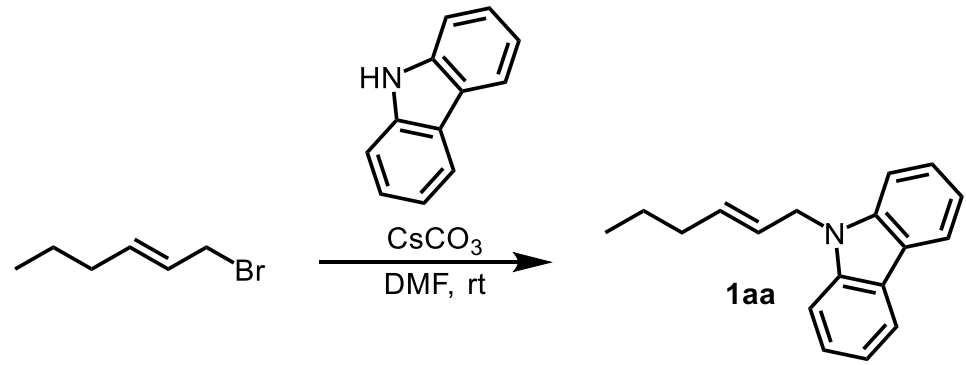

Following a literature procedure ${ }^{21}, \mathrm{Cs}_{2} \mathrm{CO}_{3}(1.95 \mathrm{~g}, 6.0 \mathrm{mmol}, 1.2$ equiv.) was added to a solution of carbazole (836 mg, $5.0 \mathrm{mmol}, 1.0$ equiv.) in DMF (50 mL). (E)-1-Bromohex-2-ene ( $0.72 \mathrm{ml}, 5.5 \mathrm{mmol}, 1.1$ equiv.) was then added. After stirring overnight, the reaction mixture was diluted with $\mathrm{CH}_{2} \mathrm{Cl}_{2}$ and quenched with $\mathrm{H}_{2} \mathrm{O}$. The phases were separated and the organics were washed with $\mathrm{H}_{2} \mathrm{O}$, dried over $\mathrm{MgSO}_{4}$, concentrated in vacuo and purified by column chromatography on silica gel (pentane/EtOAc $=20 / 1$ ) to afford the title compound 1aa as a colorless oil in 63\% yield ( $0.79 \mathrm{~g}$, eluent: petroleum ether/EtOAc $=20 / 1)$. $\mathbf{R}_{f}=0.67$ (petroleum ether $/$ EtOAc $=20 / 1$ ) 
${ }^{1} \mathrm{H}$ NMR (400 MHz, $\left.\mathrm{CDCl}_{3}\right) \delta 8.16(\mathrm{~d}, J=7.7 \mathrm{~Hz}, 2 \mathrm{H}), 7.54-7.42(\mathrm{~m}, 4 \mathrm{H}), 7.32-7.24(\mathrm{~m}, 2 \mathrm{H})$, $5.72-5.59(\mathrm{~m}, 2 \mathrm{H}), 4.91(\mathrm{~d}, J=4.0 \mathrm{~Hz}, 2 \mathrm{H}), 2.06-1.96(\mathrm{~m}, 2 \mathrm{H}), 1.41-1.32(\mathrm{~m}, 2 \mathrm{H}), 0.88(\mathrm{t}, J=$ $7.4 \mathrm{~Hz}, 3 \mathrm{H})$.

${ }^{13} \mathrm{C}$ NMR (100 MHz, $\left.\mathrm{CDCl}_{3}\right) \delta$ 140.5, 133.6, 125.7, 124.3, 123.0, 120.4, 119.0, 109.0, 44.9, 34.3, $22.3,13.7$.

HRMS (ESI-TOF) calcd for $\mathrm{C}_{18} \mathrm{H}_{20} \mathrm{~N}^{+}[\mathrm{M}+\mathrm{H}]^{+}:$250.1590, found: 250.1591 .

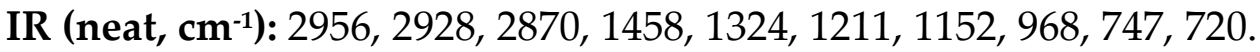

(E)-9-(Hex-2-en-1-yl)-9H-carbazole (1ab)

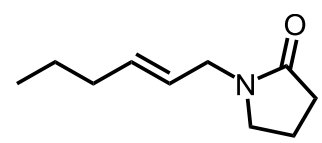

Compound $1 \mathrm{ab}$ was prepared as a colorless oil in $37 \%$ yield $(0.15 \mathrm{~g}$, eluent: $\mathrm{DCM} / \mathrm{MeOH}=$ 50/1) pyrrolidin-2-one $(0.2 \mathrm{ml}, 2.50 \mathrm{mmol}),(E)$-1-bromohex-2-ene $(0.4 \mathrm{ml}, 3.30 \mathrm{mmol})$, and $\mathrm{NaH}(60 \%, 67 \mathrm{mg}, 2.8 \mathrm{mmol})$ following the above general procedure B.

$\mathbf{R}_{f}=0.69(\mathrm{DCM} / \mathrm{MeOH}=10 / 1)$

${ }^{1} \mathrm{H}$ NMR (400 MHz, $\left.\mathrm{CDCl}_{3}\right) \delta 5.65$ - $5.55(\mathrm{~m}, 1 \mathrm{H}), 5.37-5.28(\mathrm{~m}, 1 \mathrm{H}), 3.82(\mathrm{~d}, J=6.5 \mathrm{~Hz}, 2 \mathrm{H})$, $3.32(\mathrm{t}, J=7.1 \mathrm{~Hz}, 2 \mathrm{H}), 2.39(\mathrm{t}, J=8.1 \mathrm{~Hz}, 2 \mathrm{H}), 2.04-1.95(\mathrm{~m}, 4 \mathrm{H}), 1.42-1.33(\mathrm{~m}, 2 \mathrm{H}), 0.88(\mathrm{t}, J$ $=7.4 \mathrm{~Hz}, 3 \mathrm{H})$.

${ }^{13} \mathrm{C}$ NMR (100 MHz, $\left.\mathrm{CDCl}_{3}\right) \delta$ 174.7, 134.9, 124.2, 46.7, 44.7, 34.4, 31.3, 22.4, 17.9, 13.8 .

HRMS (ESI-TOF) calcd for $\mathrm{C}_{10} \mathrm{H}_{18} \mathrm{NO}^{+}[\mathrm{M}+\mathrm{H}]^{+}:$168.1383, found: 168.1384 .

IR (neat, cm-1): 2956, 2930, 2871, 1684, 1507, 1457, 1424, 1262, 970.

tert-Butyl (E)-benzyl(hex-2-en-1-yl)carbamate (1ac)

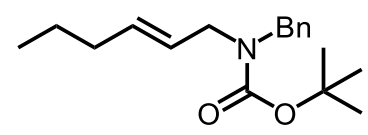

Compound 1ac was prepared as a colorless oil in $45 \%$ yield ( $0.68 \mathrm{~g}$, eluent: petroleum ether/EtOAc $=80 / 1)$ from $(E)$ - $N$-benzylhex-2-en-1-amine $(1.1 \mathrm{ml}, 5.00 \mathrm{mmol})$, di-tert-butyl pyrocarbonate $(1.4 \mathrm{ml}, 6.00 \mathrm{mmol})$, and $\mathrm{Et}_{3} \mathrm{~N}(1.0 \mathrm{ml}, 6.00 \mathrm{mmol})$ following the above general procedure F.

$\mathbf{R}_{f}=0.55$ (petroleum ether/EtOAc $=20 / 1$ )

${ }^{1}$ H NMR (400 MHz, $\mathrm{CDCl}_{3}$ ) $\delta 7.37$ - 7.21 (m, 5H), 5.63 - 5.32 (m, 2H), 4.43 (s, 2H), 3.91 - 3.55 $(\mathrm{m}, 2 \mathrm{H}), 2.02(\mathrm{q}, J=7.0 \mathrm{~Hz}, 2 \mathrm{H}), 1.50(\mathrm{~s}, 9 \mathrm{H}), 1.43-1.33(\mathrm{~m}, 2 \mathrm{H}), 0.92(\mathrm{t}, J=7.4 \mathrm{~Hz}, 3 \mathrm{H})$.

${ }^{13} \mathrm{C}$ NMR (100 MHz, $\left.\mathrm{CDCl}_{3}\right) \delta$ 155.9, 138.6, 134.1, 128.5, 128.0, 127.1, 125.4, 79.7, 49.1, 48.1, 34.4, 28.5, 22.5, 13.7. 
HRMS (ESI-TOF) calcd for $\mathrm{C}_{18} \mathrm{H}_{28} \mathrm{NO}_{2} \mathrm{Na}^{+}[\mathrm{M}+\mathrm{Na}]^{+}$: 312.1934, found: 312.1944.

IR (neat, cm$^{-1}$ ): 2961, 2930, 2871, 1694, 1457, 1411, 1364, 1240, 1165, 698.

\section{(E)-N-Benzyl-N-(hex-2-en-1-yl)pivalamide (1ad)}

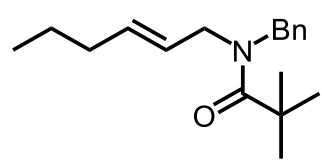

Compound 1ad was prepared as a colorless oil in $90 \%$ yield ( $0.49 \mathrm{~g}$, eluent: petroleum ether/EtOAc = 40/1) $(E)$-N-benzylhex-2-en-1-amine $(0.5 \mathrm{ml}, 2.20 \mathrm{mmol})$, PivCl (0.3 ml, 2.00 $\mathrm{mmol})$, and $\mathrm{Et}_{3} \mathrm{~N}(0.4 \mathrm{ml}, 2.50 \mathrm{mmol})$ following the above general procedure $\mathrm{F}$.

$\mathbf{R}_{f}=0.47$ (petroleum ether $/$ EtOAc $=10 / 1$ )

${ }^{1} \mathbf{H}$ NMR (400 MHz, $\left.\mathrm{CDCl}_{3}\right) \delta 7.36-7.31(\mathrm{~m}, 2 \mathrm{H}), 7.28(\mathrm{~d}, J=7.7 \mathrm{~Hz}, 1 \mathrm{H}), 7.22-7.17(\mathrm{~m}, 2 \mathrm{H})$, $5.58-5.47(\mathrm{~m}, 1 \mathrm{H}), 5.44-5.35(\mathrm{~m}, 1 \mathrm{H}), 4.64(\mathrm{~s}, 2 \mathrm{H}), 3.94(\mathrm{~d}, J=5.7 \mathrm{~Hz}, 2 \mathrm{H}), 2.08-2.01(\mathrm{~m}, 2 \mathrm{H})$, $1.45-1.38(\mathrm{~m}, 2 \mathrm{H}), 1.35(\mathrm{~s}, 9 \mathrm{H}), 0.92(\mathrm{t}, J=7.4 \mathrm{~Hz}, 3 \mathrm{H})$.

${ }^{13} \mathrm{C}$ NMR (100 MHz, $\left.\mathrm{CDCl}_{3}\right) \delta$ 177.6, 137.9, 134.6, 128.6, 127.4, 127.1, 125.0, 77.4, 48.7, 39.1, $34.3,28.7,22.4,13.7$.

HRMS (ESI-TOF) calcd for $\mathrm{C}_{18} \mathrm{H}_{28} \mathrm{NO}^{+}[\mathrm{M}+\mathrm{H}]^{+}: 274.2165$, found: 274.2174 .

IR (neat, cm$^{-1}$ ): 2926, 2857, 1461, 1096, 883, 736, 690.

\section{Compound 1ae}

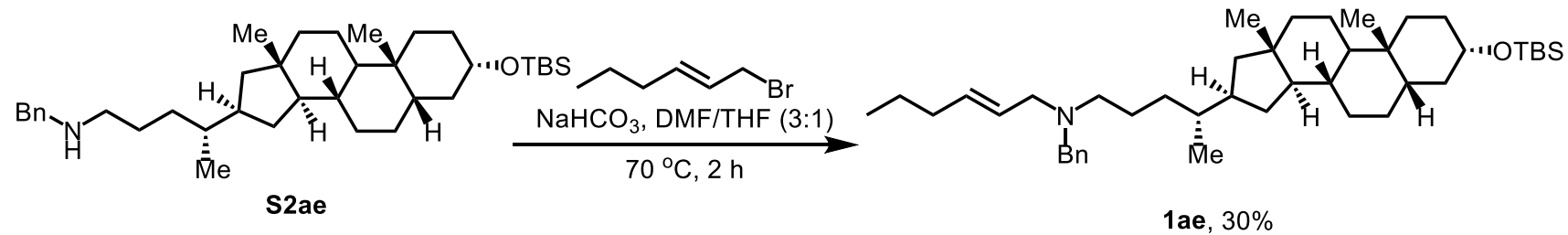

Compound 1ae was prepared as a colorless oil in 30\% yield ( $0.53 \mathrm{~g}$, eluent: petroleum ether to petroleum ether $/ \mathrm{EtOAc}=10 / 1)$ from S2ae $(1.7 \mathrm{~g}, 3.00 \mathrm{mmol}),(E)$-1-bromohex-2-ene $(0.4 \mathrm{ml}$, $3.30 \mathrm{mmol})$, and $\mathrm{NaHCO}_{3}(0.5 \mathrm{~g}, 6.00 \mathrm{mmol})$ following the above general procedure $\mathrm{G}$.

$\mathbf{R}_{f}=0.63$ (petroleum ether $/ \mathrm{EtOAc}=5 / 1$ )

${ }^{1}$ H NMR (400 MHz, $\mathrm{CDCl}_{3}$ ) $\delta 7.33$ - 7.21 (m, 5H), 5.60 - 5.45 (m, 2H), $3.74-3.44$ (m, 3H), 3.13 $-2.88(\mathrm{~m}, 2 \mathrm{H}), 2.44-2.22(\mathrm{~m}, 2 \mathrm{H}), 2.01-1.01(\mathrm{~m}, 32 \mathrm{H}), 0.90(\mathrm{t}, J=1.6 \mathrm{~Hz}, 18 \mathrm{H}), 0.62(\mathrm{~s}, 3 \mathrm{H})$, $0.06(\mathrm{~s}, 6 \mathrm{H})$.

${ }^{13} \mathrm{C}$ NMR (100 MHz, $\left.\mathrm{CDCl}_{3}\right) \delta$ 140.1, 133.8, 129.1, 128.2, 127.5, 126.8, 73.01, 58.2, 56.6, 56.4, $56.1,54.0,42.8,42.5,40.4,40.3,37.1,36.0,35.8,35.8,34.8,34.7,33.7,31.2,28.5,27.5,26.6,26.1$, 24.4, 23.6, 23.5, 22.7, 21.0, 18.8, 18.5, 13.8, 12.2, -4.4.

HRMS (ESI-TOF) calcd for $\mathrm{C}_{43} \mathrm{H}_{73} \mathrm{NONaSi}^{+}[\mathrm{M}+\mathrm{Na}]^{+}: 670.5354$, found: 670.5358 . 
IR (neat, cm-1): 2927, 2862, 1558, 1507, 1457, 1092, 1077, 834, 773, 696.

\section{Compound 1af}

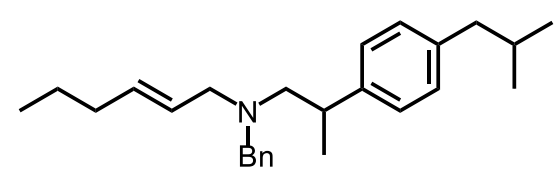

Compound 1af was prepared as a colorless oil in 69\% yield (1.0 g, eluent: DCM/EtOAc $=20 / 1)$ from $N$-benzyl-2-(4-isobutylphenyl)propan-1-amine (0.8 g, $3.00 \mathrm{mmol}),(E)-1$-bromohex-2ene $(0.4 \mathrm{ml}, 3.30 \mathrm{mmol})$, and $\mathrm{NaHCO}_{3}(0.5 \mathrm{~g}, 6.00 \mathrm{mmol})$ following the above general procedure $\mathrm{G}$.

$\mathbf{R}_{f}=0.09(\mathrm{DCM})$

${ }^{1}$ H NMR (400 MHz, $\left.\mathrm{CDCl}_{3}\right) \delta 7.36$ - $7.24(\mathrm{~m}, 5 \mathrm{H}), 7.18-7.07(\mathrm{~m}, 4 \mathrm{H}), 5.65-5.44(\mathrm{~m}, 2 \mathrm{H}), 3.70$ $(\mathrm{d}, J=13.7 \mathrm{~Hz}, 1 \mathrm{H}), 3.55(\mathrm{~d}, J=13.8 \mathrm{~Hz}, 1 \mathrm{H}), 3.20-3.08(\mathrm{~m}, 1 \mathrm{H}), 3.07-2.83(\mathrm{~m}, 2 \mathrm{H}), 2.67-$ $2.54(\mathrm{~m}, 2 \mathrm{H}), 2.52(\mathrm{~d}, J=7.2 \mathrm{~Hz}, 2 \mathrm{H}), 2.07(\mathrm{q}, J=7.0 \mathrm{~Hz}, 2 \mathrm{H}), 1.98-1.87(\mathrm{~m}, 1 \mathrm{H}), 1.52-1.40(\mathrm{~m}$, $2 \mathrm{H}), 1.31(\mathrm{~d}, J=6.9 \mathrm{~Hz}, 3 \mathrm{H}), 0.98(\mathrm{t}, J=6.9 \mathrm{~Hz}, 9 \mathrm{H})$.

${ }^{13} \mathrm{C}$ NMR (100 MHz, $\left.\mathrm{CDCl}_{3}\right) \delta$ 143.7, 140.3, 139.3, 133.6, 129.0, 128.9, 128.1, 127.6, 127.2, 126.7, $61.5,58.7,56.3,45.2,37.9,34.7,30.4,22.7,22.6,19.9,13.9$.

HRMS (ESI-TOF) calcd for $\mathrm{C}_{26} \mathrm{H}_{38} \mathrm{~N}^{+}[\mathrm{M}+\mathrm{H}]^{+}: 364.2999$, found: 364.3001 .

IR (neat, cm-1): 2961, 2930, 2871, 1694, 1411, 1240, 1165, 878, 698.

\section{Compound 1ag}

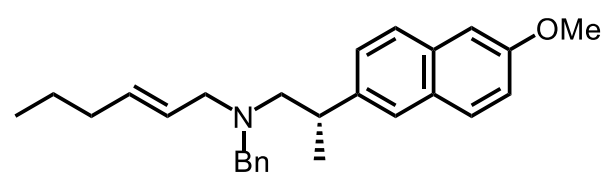

Compound 1ag was prepared as a colorless oil in $86 \%$ yield ( $0.94 \mathrm{~g}$, eluent: petroleum ether to petroleum ether/EtOAc = 10/1) from $(S)$-N-benzyl-2-(6-methoxynaphthalen-2-yl)propan1-amine (0.9 g, $2.84 \mathrm{mmol})$, (E)-1-bromohex-2-ene $(0.4 \mathrm{ml}, 3.10 \mathrm{mmol})$, and $\mathrm{NaHCO}_{3}(0.5 \mathrm{~g}$, $5.68 \mathrm{mmol})$ following the above general procedure $\mathrm{G}$.

$\mathbf{R}_{f}=0.35$ (petroleum ether $/$ EtOAc $=20 / 1$ )

${ }^{1}$ H NMR (400 MHz, $\left.\mathrm{CDCl}_{3}\right) \delta 7.42(\mathrm{~d}, J=8.5 \mathrm{~Hz}, 2 \mathrm{H}), 7.11-6.91(\mathrm{~m}, 7 \mathrm{H}), 6.89(\mathrm{~d}, J=8.7 \mathrm{~Hz}$, $2 \mathrm{H}), 5.35-5.16(\mathrm{~m}, 2 \mathrm{H}), 3.66(\mathrm{~s}, 3 \mathrm{H}), 3.39(\mathrm{~d}, J=13.7 \mathrm{~Hz}, 1 \mathrm{H}), 3.30(\mathrm{~d}, J=13.7 \mathrm{~Hz}, 1 \mathrm{H}), 2.93-$ $2.70(\mathrm{~m}, 3 \mathrm{H}), 2.48-2.38(\mathrm{~m}, 1 \mathrm{H}), 2.38-2.25(\mathrm{~m}, 1 \mathrm{H}), 1.77(\mathrm{q}, J=7.0 \mathrm{~Hz}, 2 \mathrm{H}), 1.25-1.11(\mathrm{~m}$, $2 \mathrm{H}), 1.08(\mathrm{~d}, J=6.9 \mathrm{~Hz}, 3 \mathrm{H}), 0.68(\mathrm{t}, J=7.4 \mathrm{~Hz}, 3 \mathrm{H})$.

${ }^{13} \mathrm{C}$ NMR (100 MHz, $\left.\mathrm{CDCl}_{3}\right) \delta$ 157.2, 141.7, 140.2, 133.7, 133.3, 129.19, 129.17, 128.9, 128.1, $127.5,126.9,126.7,126.6,125.4,118.6,105.7,61.2,58.7,56.4,55.4,38.2,34.7,22.7,20.1,13.9$. 
HRMS (ESI-TOF) calcd for $\mathrm{C}_{27} \mathrm{H}_{34} \mathrm{NO}^{+}[\mathrm{M}+\mathrm{H}]^{+}$: 388.2635, found: 388.2641.

IR (neat, cm-1): 2957, 2926, 2793, 1348, 1606, 1507, 11457, 1263, 1033, 849, 737, 698.

\section{Co-Catalyzed Remote Hydroboration of Alkenyl Amines}

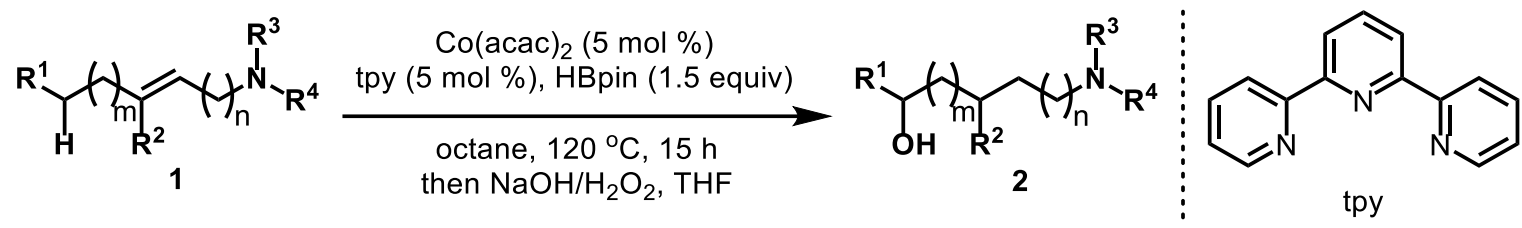

\section{General Procedure H:}

In a glove box, to an oven-dried 10-mL vial were added Co(acac)2 (6.5 mg, $0.025 \mathrm{mmol})$, tpy $(5.9 \mathrm{mg}, 0.025 \mathrm{mmol})$ and anhydrous octane $(2.0 \mathrm{~mL})$. The resulting solution was stirred for $20 \mathrm{~min}$ at room temperature, at which time HBpin $(96.1 \mathrm{mg}, 0.75 \mathrm{mmol})$ was added. After the resulting mixture was stirred for an additional $5 \mathrm{~min}$, alkenyl amine $(0.5 \mathrm{mmol})$ was added and stirred for $15 \mathrm{~h}$ at $120{ }^{\circ} \mathrm{C}$. The resulting suspension was cooled to room temperature, filtered through a celite pad with EtOAc and concentrated under vacuo. The residue was dissolved in THF $(2.0 \mathrm{~mL})$ and cooled to $0{ }^{\circ} \mathrm{C}$. $\mathrm{NaOH}(1.5$ equiv, $3.0 \mathrm{M})$ and $\mathrm{H}_{2} \mathrm{O}_{2}$ (1.5 equiv, 30\%) were then added. The resulting mixture was stirred for $0.5 \mathrm{~h}$, and extracted with EtOAc, dried with $\mathrm{Na}_{2} \mathrm{SO}_{4}$, filtered and concentrated. The residue was purified by silica gel chromatography to afford the desired products.

\section{General Procedure I (purified by base-acid extraction):}

In a glove box, to an oven-dried 10-mL vial were added Co(acac)2 $(6.5 \mathrm{mg}, 0.025 \mathrm{mmol})$, tpy $(5.9 \mathrm{mg}, 0.025 \mathrm{mmol})$ and anhydrous octane $(2.0 \mathrm{~mL})$. The resulting solution was stirred for $20 \mathrm{~min}$ at room temperature, at which time HBpin $(96.1 \mathrm{mg}, 0.75 \mathrm{mmol})$ was added. After the resulting mixture was stirred for an additional $5 \mathrm{~min}$, allylic amine $(0.5 \mathrm{mmol})$ was added and stirred for $15 \mathrm{~h}$ at $120{ }^{\circ} \mathrm{C}$. The resulting suspension was cooled to room temperature, filtered through a celite pad with EtOAc and concentrated under vacuo. The crude material was acidified by $\mathrm{HCl}$ (aq.) and extracted with EtOAc. The aqueous layer was then basified by $\mathrm{NaOH}$ (aq.) and extracted with EtOAc. The combined organic layers were dried with $\mathrm{Na}_{2} \mathrm{SO}_{4}$ and concentrated to give the crude amine. The residue was dissolved in THF $(2.0 \mathrm{~mL})$ 
and cooled to $0{ }^{\circ} \mathrm{C}$. $\mathrm{NaOH}(1.5$ equiv, $3.0 \mathrm{M})$ and $\mathrm{H}_{2} \mathrm{O}_{2}(1.5$ equiv, 30\%) were added. The resulting mixture was stirred for $0.5 \mathrm{~h}$, and extracted with EtOAc. The organic layer was dried over $\mathrm{Na}_{2} \mathrm{SO}_{4}$, filtered, concentrated to give the crude aminoalcohol. The aminoalcohol was purified by base-acid extraction to give the desired products.

\section{General Procedure $\mathrm{J}\left(\mathrm{NaBO}_{3} \cdot 4 \mathrm{H}_{2} \mathrm{O}\right.$ as oxidant):}

In a glove box, to an oven-dried 10-mL vial were added Co(acac)2 $(6.5 \mathrm{mg}, 0.025 \mathrm{mmol})$, tpy $(5.9 \mathrm{mg}, 0.025 \mathrm{mmol})$ and anhydrous octane $(2.0 \mathrm{~mL})$. The resulting solution was stirred for $20 \mathrm{~min}$ at room temperature, at which time HBpin $(96.1 \mathrm{mg}, 0.75 \mathrm{mmol})$ was added. After the resulting mixture was stirred for an additional $5 \mathrm{~min}$, allylic amine $(0.5 \mathrm{mmol})$ was added and stirred for $15 \mathrm{~h}$ at $120^{\circ} \mathrm{C}$. The resulting suspension was cooled to room temperature, filtered through a celite pad with EtOAc and concentrated under vacuo. The residue was dissolved in THF $(1.0 \mathrm{~mL})$ and $\mathrm{H}_{2} \mathrm{O}(1.0 \mathrm{ml})$, and $\mathrm{NaBO}_{3} \cdot 4 \mathrm{H}_{2} \mathrm{O}(3.0$ equiv) was then added. The resulting mixture was stirred for $6 \mathrm{~h}$ at room temperature, and extracted with EtOAc, dried with $\mathrm{Na}_{2} \mathrm{SO}_{4}$, filtered and concentrated. The residue was purified by silica gel chromatography to afford the desired products.

\section{6-(Dibenzylamino)hexan-1-ol (2a)}

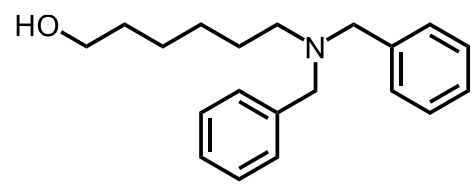

Compound 2a was prepared as a colorless oil in 77\% yield (114.3 mg, eluent: petroleum ether/EtOAc = 5/1), from $(E)$ - $N, N$-dibenzylhex-2-en-1-amine 1a (138.5 mg, $0.50 \mathrm{mmol}$ ), $\mathrm{Co}(\mathrm{acac})_{2}(6.5 \mathrm{mg}, 0.025 \mathrm{mmol})$, tpy $(5.9 \mathrm{mg}, 0.025 \mathrm{mmol})$, and HBpin $(96.0 \mathrm{mg}, 0.75 \mathrm{mmol})$ following the above general procedure $\mathrm{H}$.

$\mathbf{R}_{f}=0.18$ (petroleum ether/EtOAc $=5 / 1$ )

${ }^{1}$ H NMR (400 MHz, $\left.\mathrm{CDCl}_{3}\right) \delta 7.37(\mathrm{~d}, J=8.2 \mathrm{~Hz}, 4 \mathrm{H}), 7.35-7.26(\mathrm{~m}, 4 \mathrm{H}), 7.26-7.20(\mathrm{~m}, 2 \mathrm{H})$, $3.59(\mathrm{t}, J=6.6 \mathrm{~Hz}, 2 \mathrm{H}), 3.55(\mathrm{~s}, 4 \mathrm{H}), 2.47-2.37(\mathrm{~m}, 2 \mathrm{H}), 1.56-1.48(\mathrm{~m}, 4 \mathrm{H}), 1.29-1.24(\mathrm{~m}, 4 \mathrm{H})$. ${ }^{13} \mathrm{C}$ NMR (100 MHz, $\left.\mathrm{CDCl}_{3}\right) \delta 140.1,128.9,128.3,126.9,63.1,58.5,53.3,32.9,27.1,27.1,25.6$. The ${ }^{1} \mathrm{H}$ and ${ }^{13} \mathrm{C}$ NMR spectroscopic data match the reported characterization data. ${ }^{16}$

\section{4-(Dibenzylamino)butan-1-ol (2b)}




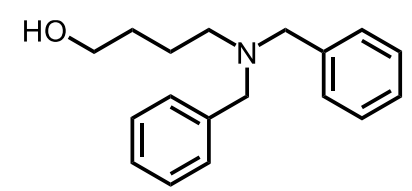

Compound $\mathbf{2} \mathbf{b}$ was prepared as a colorless oil in $53 \%$ yield $(71.9 \mathrm{mg}$, eluent: petroleum ether/EtOAc $=5 / 1)$, from $(E)$-N,N-dibenzylbut-2-en-1-amine $1 \mathbf{b}(125.7 \mathrm{mg}, 0.50 \mathrm{mmol})$, Co(acac) $2(6.5 \mathrm{mg}, 0.025 \mathrm{mmol})$, tpy (5.9 mg, $0.025 \mathrm{mmol})$, and HBpin $(96.0 \mathrm{mg}, 0.75 \mathrm{mmol})$ following the above general procedure $\mathrm{H}$.

$\mathbf{R}_{f}=0.28$ (petroleum ether $/ \mathrm{EtOAc}=5 / 1$ )

${ }^{1} \mathrm{H}$ NMR (400 MHz, $\left.\mathrm{CDCl}_{3}\right) \delta 7.39-7.33(\mathrm{~m}, 8 \mathrm{H}), 7.30-7.26(\mathrm{~m}, 2 \mathrm{H}), 3.60(\mathrm{~s}, 4 \mathrm{H}), 3.58(\mathrm{t}, J=$ $5.7 \mathrm{~Hz}, 2 \mathrm{H}), 2.50(\mathrm{t}, J=6.2 \mathrm{~Hz}, 2 \mathrm{H}), 1.71-1.56(\mathrm{~m}, 4 \mathrm{H})$.

${ }^{13} \mathrm{C}$ NMR (100 MHz, $\mathrm{CDCl}_{3}$ ) $\delta$ 138.55, 129.40, 128.40, 127.22, 62.78, 58.46, 53.85, 31.48, 24.67.

The ${ }^{1} \mathrm{H}$ and ${ }^{13} \mathrm{C}$ NMR spectroscopic data match the reported characterization data. ${ }^{22}$

\section{1-(Dibenzylamino)undecan-1-ol (2c)}

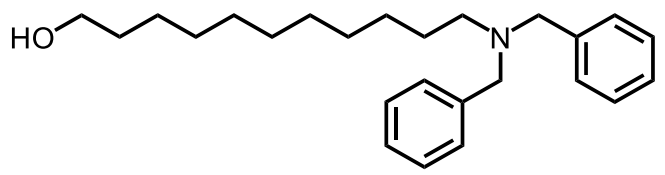

Compound 2c was prepared as a colorless oil in 50\% yield (90.9 mg, eluent: petroleum ether/EtOAc $=10 / 1)$, from $(E)-N, N$-dibenzylundec-2-en-1-amine $1 c(173.5 \mathrm{mg}, 0.50 \mathrm{mmol})$, Co(acac)2 ( $6.5 \mathrm{mg}, 0.025 \mathrm{mmol})$, tpy (5.9 mg, $0.025 \mathrm{mmol})$, and HBpin $(96.0 \mathrm{mg}, 0.75 \mathrm{mmol})$ following the above general procedure $\mathrm{H}$.

$\mathbf{R}_{f}=0.32$ (petroleum ether $/ \mathrm{EtOAc}=10 / 1$ )

${ }^{1} \mathrm{H}$ NMR $\left(400 \mathrm{MHz}, \mathrm{CDCl}_{3}\right)$ d $7.41-7.35(\mathrm{~m}, 4 \mathrm{H}), 7.34-7.27(\mathrm{~m}, 4 \mathrm{H}), 7.26-7.19(\mathrm{~m}, 2 \mathrm{H}), 3.64$ $(\mathrm{t}, J=6.6 \mathrm{~Hz}, 2 \mathrm{H}), 3.55(\mathrm{~s}, 4 \mathrm{H}), 2.45-2.37(\mathrm{~m}, 2 \mathrm{H}), 1.59-1.48(\mathrm{~m}, 4 \mathrm{H}), 1.33-1.19(\mathrm{~m}, 14 \mathrm{H})$.

${ }^{13} \mathrm{C}$ NMR (100 MHz, $\left.\mathrm{CDCl}_{3}\right) \delta$ 140.2, 128.9, 128.2, 126.8, 63.2, 58.4, 53.6, 33.0, 29.7, 29.73, 29.7, 29.6, 29.6, 27.4, 27.1, 25.9.

HRMS (ESI-TOF) calcd for $\mathrm{C}_{25} \mathrm{H}_{38} \mathrm{NO}^{+}[\mathrm{M}+\mathrm{H}]^{+}: 268.2948$, found: 268.2949 .

IR (neat, cm-1): 3649, 2925, 2853, 1684, 1558, 1507, 743, 679.

6-(Benzyl(methyl)amino)hexan-1-ol (2d)

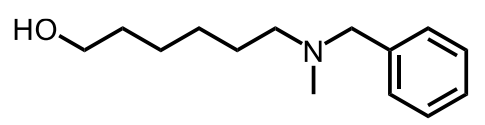

Compound $2 \mathbf{d}$ was prepared as a colorless oil in $69 \%$ yield $(66.4 \mathrm{mg}$, eluent: $\mathrm{DCM} / \mathrm{MeOH}=$ 40/1), from (E)-N-benzyl- $N$-methylhex-2-en-1-amine 1d (101.6 mg, $0.50 \mathrm{mmol})$, $\mathrm{Co}(\mathrm{acac})_{2}(6.5$ $\mathrm{mg}$, $0.025 \mathrm{mmol})$, tpy $(5.9 \mathrm{mg}, 0.025 \mathrm{mmol})$, and HBpin $(96.0 \mathrm{mg}, 0.75 \mathrm{mmol})$ following the 
above general procedure $\mathrm{H}$.

$\mathbf{R}_{f}=0.41(\mathrm{DCM} / \mathrm{MeOH}=10 / 1)$

${ }^{1}$ H NMR (400 MHz, $\left.\mathrm{CDCl}_{3}\right) \delta 7.34(\mathrm{~d}, J=4.3 \mathrm{~Hz}, 4 \mathrm{H}), 7.31-7.22(\mathrm{~m}, 1 \mathrm{H}), 3.63(\mathrm{t}, J=6.6 \mathrm{~Hz}$, 2H), $3.54(\mathrm{~s}, 2 \mathrm{H}), 2.46-2.37(\mathrm{~m}, 2 \mathrm{H}), 2.23(\mathrm{~s}, 3 \mathrm{H}), 1.62-1.52(\mathrm{~m}, 4 \mathrm{H}), 1.41-1.32(\mathrm{~m}, 4 \mathrm{H})$.

${ }^{13} \mathrm{C}$ NMR (100 MHz, $\left.\mathrm{CDCl}_{3}\right) \delta$ 138.4, 129.4, 128.4, 127.2, 62.8, 62.2, 57.3, 42.2, 32.8, 27.2, 27.1, 25.7.

The ${ }^{1} \mathrm{H}$ and ${ }^{13} \mathrm{C}$ NMR spectroscopic data match the reported characterization data. ${ }^{23}$

\section{6-(Dibutylamino)hexan-1-ol (2e)}<smiles>CN(CCCCCCCO)C(C)(C)C</smiles>

Compound 2e was prepared as a yellow oil in 32\% yield (37.1 mg), from $(E)-N, N$-dibutylhex2-en-1-amine 1e (105.6 mg, $0.50 \mathrm{mmol}), \mathrm{Co}($ acac)2 $(6.5 \mathrm{mg}, 0.025 \mathrm{mmol})$, tpy (5.9 mg, 0.025 $\mathrm{mmol})$, and HBpin $(96.0 \mathrm{mg}, 0.75 \mathrm{mmol})$ following the above general procedure $\mathrm{I}$.

${ }^{1} \mathbf{H}$ NMR (400 MHz, $\left.\mathrm{CDCl}_{3}\right) \delta 3.64(\mathrm{t}, J=6.6 \mathrm{~Hz}, 2 \mathrm{H}), 2.41(\mathrm{~m}, 6 \mathrm{H}), 1.45-1.37(\mathrm{~m}, 8 \mathrm{H}), 1.33$ $1.23(\mathrm{~m}, 8 \mathrm{H}), 0.90(\mathrm{t}, J=7.4 \mathrm{~Hz}, 6 \mathrm{H})$.

${ }^{13} \mathrm{C}$ NMR (100 MHz, $\left.\mathrm{CDCl}_{3}\right) \delta$ 63.0, 54.1, 53.9, 32.9, 29.0, 27.4, 26.9, 25.8, 20.9, 14.2.

HRMS (ESI-TOF) calcd for $\mathrm{C}_{14} \mathrm{H}_{32} \mathrm{NO}^{+}[\mathrm{M}+\mathrm{H}]^{+}:$230.2478, found: 230.2487 .

IR (neat, cm-1): 3143, 2956, 2930, 2862, 2362, 1558, 1507, 744.

Methyl 1-(6-hydroxyhexyl)piperidine-4-carboxylate (2f)

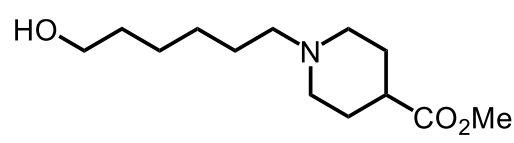

Compound $2 \mathrm{f}$ was prepared as a colorless oil in $47 \%$ yield $(61.7 \mathrm{mg}$, eluent: $\mathrm{DCM} / \mathrm{MeOH}=$ 40/1), from methyl (E)-1-(hex-2-en-1-yl)piperidine-4-carboxylate 1f (112.6 mg, $0.50 \mathrm{mmol})$, Co(acac)2 $(6.5 \mathrm{mg}, 0.025 \mathrm{mmol})$, tpy $(5.9 \mathrm{mg}, 0.025 \mathrm{mmol})$, and HBpin $(96.0 \mathrm{mg}, 0.75 \mathrm{mmol})$ following the above general procedure $\mathrm{J}$.

$\mathbf{R}_{f}=0.47(\mathrm{DCM} / \mathrm{MeOH}=10 / 1)$

${ }^{1} \mathrm{H}$ NMR (400 MHz, $\left.\mathrm{CDCl}_{3}\right) \delta 3.66(\mathrm{~s}, 3 \mathrm{H}), 3.62-3.59(\mathrm{~m}, 2 \mathrm{H}), 2.89-2.82(\mathrm{~m}, 2 \mathrm{H}), 2.33-2.25$ $(\mathrm{m}, 3 \mathrm{H}), 2.03-1.95(\mathrm{~m}, 2 \mathrm{H}), 1.91-1.86(\mathrm{~m}, 2 \mathrm{H}), 1.81-1.72(\mathrm{~m}, 2 \mathrm{H}), 1.57-1.46(\mathrm{~m}, 4 \mathrm{H}), 1.38$ $-1.28(\mathrm{~m}, 4 \mathrm{H})$.

${ }^{13} \mathrm{C}$ NMR (100 MHz, $\left.\mathrm{CDCl}_{3}\right) \delta$ 175.6, 62.8, 58.9, 53.1, 51.7, 41.1, 32.7, 28.2, 27.4, 26.8, 25.7.

HRMS (ESI-TOF) calcd for $\mathrm{C}_{13} \mathrm{H}_{26} \mathrm{NO}_{3}{ }^{+}[\mathrm{M}+\mathrm{H}]^{+}:$244.1907, found: 244.1917 .

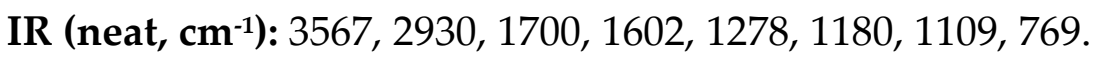


Methyl (R)-1-(6-hydroxyhexyl)piperidine-3-carboxylate (2g)

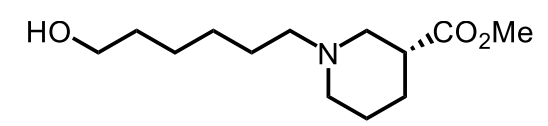

Compound $2 \mathrm{~g}$ was prepared as a colorless oil in $52 \%$ yield $(67.9 \mathrm{mg}$, eluent: $\mathrm{DCM} / \mathrm{MeOH}=$ 40/1), from methyl $(R, E)-1$-(hex-2-en-1-yl)piperidine-3-carboxylate $1 \mathrm{~g}$ (112.6 mg, $0.50 \mathrm{mmol})$, Co(acac)2 $(6.5 \mathrm{mg}, 0.025 \mathrm{mmol})$, tpy $(5.9 \mathrm{mg}, 0.025 \mathrm{mmol})$, and HBpin $(96.0 \mathrm{mg}, 0.75 \mathrm{mmol})$ following the above general procedure $\mathrm{J}$.

$\mathbf{R}_{f}=0.50(\mathrm{DCM} / \mathrm{MeOH}=10 / 1)$

${ }^{1}$ H NMR (400 MHz, $\left.\mathrm{CDCl}_{3}\right) \delta 3.66(\mathrm{~s}, 3 \mathrm{H}), 3.61(\mathrm{t}, J=6.5 \mathrm{~Hz}, 2 \mathrm{H}), 3.08-3.00(\mathrm{~m}, 1 \mathrm{H}), 2.85$ $2.79(\mathrm{~m}, 1 \mathrm{H}), 2.66-2.60(\mathrm{~m}, 1 \mathrm{H}), 2.39-2.34(\mathrm{~m}, 2 \mathrm{H}), 2.14(\mathrm{t}, J=10.9 \mathrm{~Hz}, 1 \mathrm{H}), 2.02-1.93(\mathrm{~m}$, $2 \mathrm{H}), 1.66-1.30(\mathrm{~m}, 11 \mathrm{H})$.

${ }^{13} \mathrm{C}$ NMR (100 MHz, $\left.\mathrm{CDCl}_{3}\right) \delta$ 174.6, 62.8, 58.9, 55.3, 53.8, 51.8, 41.6, 32.7, 27.3, 27.0, 26.5, 25.6, 24.4.

HRMS (ESI-TOF) calcd for $\mathrm{C}_{13} \mathrm{H}_{26} \mathrm{NO}_{3}{ }^{+}[\mathrm{M}+\mathrm{H}]^{+}:$244.1907, found: 244.1914 .

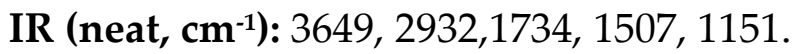

Methyl (6-hydroxyhexyl)-L-prolinate (2h)

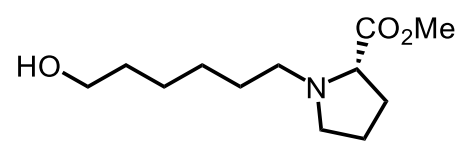

Compound $\mathbf{2 h}$ was prepared as a colorless oil in 39\% yield (45.0 mg, eluent: DCM/MeOH = 40/1), from methyl (E)-hex-2-en-1-yl- $L$-prolinate 1 h (105.5 mg, $0.50 \mathrm{mmol}), \mathrm{Co}($ acac)2 (6.5 mg, $0.025 \mathrm{mmol})$, tpy (5.9 mg, $0.025 \mathrm{mmol})$, and HBpin $(96.0 \mathrm{mg}, 0.75 \mathrm{mmol})$ following the above general procedure J.

$\mathbf{R}_{f}=0.61(\mathrm{DCM} / \mathrm{MeOH}=10 / 1)$

${ }^{1} \mathrm{H}$ NMR (400 MHz, CDCl N $_{3} \delta .70$ (s, 3H), 3.60 (t, J = 6.6 Hz, 2H), $3.20-3.09$ (m, 2H), 2.68 $2.60(\mathrm{~m}, 1 \mathrm{H}), 2.39-2.27(\mathrm{~m}, 2 \mathrm{H}), 2.13-2.06(\mathrm{~m}, 1 \mathrm{H}), 1.93-1.86(\mathrm{~m}, 2 \mathrm{H}), 1.82-1.73(\mathrm{~m}, 1 \mathrm{H})$, $1.57-1.45(\mathrm{~m}, 4 \mathrm{H}), 1.35-1.25(\mathrm{~m}, 4 \mathrm{H})$.

${ }^{13} \mathrm{C}$ NMR (100 MHz, $\left.\mathrm{CDCl}_{3}\right) \delta$ 174.9, 66.3, 62.9, 55.2, 53.6, 52.0, 32.7, 29.4, 28.6, 27.3, 25.6, 23.2. HRMS (ESI-TOF) calcd for $\mathrm{C}_{12} \mathrm{H}_{24} \mathrm{NO}_{3}{ }^{+}[\mathrm{M}+\mathrm{H}]^{+}:$230.1751, found: 230.1760 .

IR (neat, cm ${ }^{-1}$ ): 3414, 2930, 1734, 1457, 1197, 1171, 1082, 1057.

6-Morpholinohexan-1-ol (2i) 


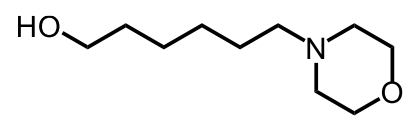

Compound $2 \mathbf{i}$ was prepared as a colorless oil in $68 \%$ yield $(63.5 \mathrm{mg}$, eluent: $\mathrm{DCM} / \mathrm{MeOH}=$ 40/1), from (E)-4-(hex-2-en-1-yl)morpholine 1i ( $84.6 \mathrm{mg}$, $0.50 \mathrm{mmol}$ ), Co(acac)2 (6.5 mg, 0.025 mmol), tpy $(5.9 \mathrm{mg}, 0.025 \mathrm{mmol})$, and HBpin $(96.0 \mathrm{mg}$, $0.75 \mathrm{mmol})$ following the above general procedure $\mathrm{H}$.

$\mathbf{R}_{f}=0.35(\mathrm{DCM} / \mathrm{MeOH}=10 / 1)$

${ }^{1} \mathrm{H}$ NMR $\left(400 \mathrm{MHz}, \mathrm{CDCl}_{3}\right) \delta 3.71(\mathrm{t}, J=4.5 \mathrm{~Hz}, 4 \mathrm{H}), 3.62(\mathrm{t}, J=6.5 \mathrm{~Hz}, 2 \mathrm{H}), 2.45-2.43(\mathrm{~m}$, $4 \mathrm{H}), 2.35-2.31(\mathrm{~m}, 2 \mathrm{H}), 1.59-1.46(\mathrm{~m}, 4 \mathrm{H}), 1.39-1.29(\mathrm{~m}, 4 \mathrm{H})$.

${ }^{13} \mathrm{C}$ NMR (100 MHz, $\left.\mathrm{CDCl}_{3}\right)$ ठ 67.0, 62.9, 59.1, 53.8, 32.7, 27.3, 26.4, 25.7.

The ${ }^{1} \mathrm{H}$ and ${ }^{13} \mathrm{C}$ NMR spectroscopic data match the reported characterization data. ${ }^{24}$

6-(1,4-Dioxa-8-azaspiro[4.5]decan-8-yl)hexan-1-ol (2j)

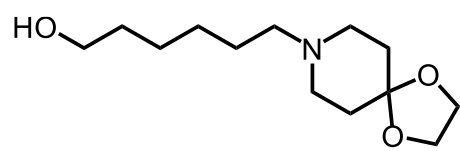

Compound $2 \mathbf{j}$ was prepared as a colorless oil in $62 \%$ yield $(71.7 \mathrm{mg}$, eluent: $\mathrm{DCM} / \mathrm{MeOH}=$ 40/1), from (E)-8-(hex-2-en-1-yl)-1,4-dioxa-8-azaspiro[4.5]decane $\mathbf{1 j}$ (112.6 mg, $0.50 \mathrm{mmol}$ ), Co(acac) $2(6.5 \mathrm{mg}, 0.025 \mathrm{mmol})$, tpy $(5.9 \mathrm{mg}, 0.025 \mathrm{mmol})$, and HBpin $(96.0 \mathrm{mg}, 0.75 \mathrm{mmol})$ following the above general procedure $\mathrm{H}$.

$\mathbf{R}_{f}=0.21(\mathrm{DCM} / \mathrm{MeOH}=10 / 1)$

${ }^{1} \mathrm{H}$ NMR $\left(400 \mathrm{MHz}, \mathrm{CDCl}_{3}\right) \delta 3.94(\mathrm{~s}, 4 \mathrm{H}), 3.62(\mathrm{t}, J=6.5 \mathrm{~Hz}, 2 \mathrm{H}), 2.59(\mathrm{~s}, 4 \mathrm{H}), 2.43-2.37(\mathrm{~m}$, $2 \mathrm{H}), 1.79(\mathrm{t}, J=5.8 \mathrm{~Hz}, 4 \mathrm{H}), 1.59-1.51(\mathrm{~m}, 4 \mathrm{H}), 1.40-1.30(\mathrm{~m}, 4 \mathrm{H})$.

${ }^{13} \mathrm{C}$ NMR (100 MHz, $\left.\mathrm{CDCl}_{3}\right) \delta$ d 107.1, 64.4, 62.9, 58.2, 51.4, 34.6, 32.7, 27.3, 26.9, 25.6.

HRMS (ESI-TOF) calcd for $\mathrm{C}_{13} \mathrm{H}_{26} \mathrm{NO}_{3}{ }^{+}[\mathrm{M}+\mathrm{H}]^{+}: 244.1907$, found: 244.1916 .

IR (neat, cm-1): 3385, 2930, 2363, 1653, 1558, 1090, 1040, 744.

\section{5-(Dibenzylamino)-1-phenylpentan-1-ol (2k)}<smiles>OC(CCCCN(Cc1ccccc1)Cc1ccccc1)c1ccccc1</smiles>

Compound 2k was prepared as a colorless oil in $92 \%$ yield (165.9 mg, eluent: petroleum ether/EtOAc $=10 / 1)$, from $(E)$ - N,N-dibenzyl-5-phenylpent-2-en-1-amine $1 \mathbf{k}(170.5 \mathrm{mg}, 0.50$ $\mathrm{mmol}), \mathrm{Co}(\mathrm{acac}) 2(6.5 \mathrm{mg}, 0.025 \mathrm{mmol})$, tpy $(5.9 \mathrm{mg}, 0.025 \mathrm{mmol})$, and HBpin $(96.0 \mathrm{mg}, 0.75$ $\mathrm{mmol}$ ) following the above general procedure $\mathrm{H}$. 
$\mathbf{R}_{f}=0.53$ (petroleum ether $/$ EtOAc $=5 / 1$ )

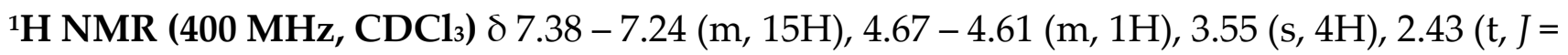
$7.1 \mathrm{~Hz}, 2 \mathrm{H}), 1.75-1.32(\mathrm{~m}, 6 \mathrm{H})$.

${ }^{13} \mathrm{C}$ NMR (100 MHz, $\left.\mathrm{CDCl}_{3}\right) \delta$ 144.9, 140.1, 128.9, 128.5, 128.3, 127.6, 126.8, 126.1, 74.71, 58.5, 53.2, 39.0, 27.0, 23.5.

HRMS (ESI-TOF) calcd for $\mathrm{C}_{25} \mathrm{H}_{30} \mathrm{NO}^{+}[\mathrm{M}+\mathrm{H}]^{+}:$360.2322, found: 320.2330 .

IR (neat, cm ${ }^{-1}$ ): 3649, 3030, 2930, 1684, 1507, 1027, 744, 697.

\section{4-(Benzyl(methyl)amino)-2-methylbutan-1-ol (21)}

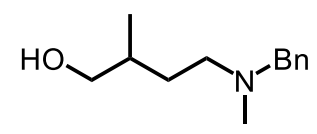

Compound 21 was prepared as a colorless oil in 49\% yield $(51.0 \mathrm{mg}$, eluent: DCM/EtOAc $=$ 10/1), from $N$-benzyl-N,3-dimethylbut-2-en-1-amine 11 (95.1 mg, 0.50 mmol), Co(acac)2 (6.5 $\mathrm{mg}, 0.025 \mathrm{mmol})$, tpy $(5.9 \mathrm{mg}, 0.025 \mathrm{mmol})$, and HBpin $(96.0 \mathrm{mg}, 0.75 \mathrm{mmol})$ following the above general procedure $\mathrm{H}$.

$\mathbf{R}_{f}=0.70$ (petroleum ether $/$ EtOAc $=10 / 1$ )

${ }^{1}$ H NMR (400 MHz, $\mathrm{CDCl}_{3}$ ) $\delta 7.37$ - 7.27 (m, 5H), 3.63 - 3.49 (m, 3H), 3.39 - 3.30 (m, 1H), 2.63 $-2.43(\mathrm{~m}, 2 \mathrm{H}), 2.19(\mathrm{~s}, 3 \mathrm{H}), 1.84-1.54(\mathrm{~m}, 3 \mathrm{H}), 0.91(\mathrm{~d}, J=6.8 \mathrm{~Hz}, 3 \mathrm{H})$.

${ }^{13} \mathrm{C}$ NMR (100 MHz, $\left.\mathrm{CDCl}_{3}\right) \delta$ 137.2, 129.6, 128.5, 127.5, 68.5, 62.6, 56.3, 41.1, 36.7, 33.8, 18.2.

The ${ }^{1} \mathrm{H}$ and ${ }^{13} \mathrm{C}$ NMR spectroscopic data match the reported characterization data. ${ }^{25}$

\section{(1-Benzylpiperidin-4-yl)methanol (2m)}<smiles>OCC1CCN(Br)CC1</smiles>

Compound $2 \mathrm{~m}$ was prepared as a colorless oil in $75 \%$ yield $(77.0 \mathrm{mg}$, eluent: $\mathrm{DCM} / \mathrm{MeOH}=$ 40/1), from 1-benzyl-4-methyl-1,2,3,6-tetrahydropyridine 1m (93.5 mg, 0.50 mmol), Co(acac)2 (6.5 mg, $0.025 \mathrm{mmol})$, tpy (5.9 mg, $0.025 \mathrm{mmol})$, and HBpin (96.0 mg, $0.75 \mathrm{mmol})$ following the above general procedure $\mathrm{H}$.

$\mathbf{R}_{f}=0.30(\mathrm{DCM} / \mathrm{MeOH}=10 / 1)$

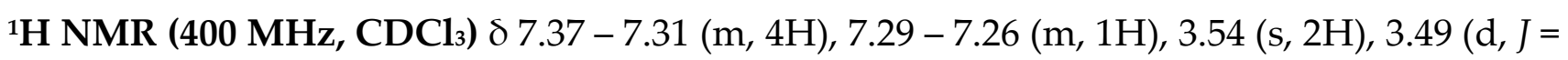


$6.4 \mathrm{~Hz}, 2 \mathrm{H}), 3.00-2.91(\mathrm{~m}, 2 \mathrm{H}), 2.55(\mathrm{~s}, 1 \mathrm{H}), 2.04-1.95(\mathrm{~m}, 2 \mathrm{H}), 1.77-1.69(\mathrm{~m}, 2 \mathrm{H}), 1.58-$ $1.47(\mathrm{~m}, 1 \mathrm{H}), 1.34-1.28(\mathrm{~m}, 2 \mathrm{H})$.

${ }^{13} \mathrm{C}$ NMR (100 MHz, $\left.\mathrm{CDCl}_{3}\right) \delta$ 138.1, 129.5, 128.3, 127.2, 67.9, 63.5, 53.5, 38.6, 28.8.

The ${ }^{1} \mathrm{H}$ and ${ }^{13} \mathrm{C}$ NMR spectroscopic data match the reported characterization data. ${ }^{26}$ 6-(Methyl(phenyl)amino)hexan-1-ol (2n)<smiles>CN(CCCCCCO)c1ccccc1</smiles>

Compound $2 \mathrm{n}$ was prepared as a colorless oil in $68 \%$ yield $(70.9 \mathrm{mg}$, eluent: petroleum ether/EtOAc = 5/1), from $(E)-N$-(hex-2-en-1-yl)- $N$-methylaniline $1 n$ (95.1 mg, $0.50 \mathrm{mmol})$, $\mathrm{Co}(\mathrm{acac})_{2}(6.5 \mathrm{mg}, 0.025 \mathrm{mmol})$, tpy $(5.9 \mathrm{mg}, 0.025 \mathrm{mmol})$, and HBpin $(96.0 \mathrm{mg}, 0.75 \mathrm{mmol})$ following the above general procedure $\mathrm{H}$.

$\mathbf{R}_{f}=0.25$ (petroleum ether $/$ EtOAc $=5 / 1$ )

${ }^{1}$ H NMR (400 MHz, $\left.\mathrm{CDCl}_{3}\right) \delta 7.28-7.22(\mathrm{~m}, 2 \mathrm{H}), 6.74-6.68(\mathrm{~m}, 3 \mathrm{H}), 3.66(\mathrm{t}, J=6.6 \mathrm{~Hz}, 2 \mathrm{H})$, $3.35-3.30(\mathrm{~m}, 2 \mathrm{H}), 2.94(\mathrm{~s}, 3 \mathrm{H}), 1.65-1.57(\mathrm{~m}, 4 \mathrm{H}), 1.43-1.34(\mathrm{~m}, 4 \mathrm{H})$.

${ }^{13} \mathrm{C}$ NMR (100 MHz, $\left.\mathrm{CDCl}_{3}\right) \delta$ 149.4, 129.3, 116.0, 112.2, 63.0, 52.8, 38.4, 32.9, 27.1, 26.7, 25.8.

The ${ }^{1} \mathrm{H}$ and ${ }^{13} \mathrm{C}$ NMR spectroscopic data match the reported characterization data. ${ }^{27}$

\section{6-(Ethyl(m-tolyl)amino)hexan-1-ol (20)}<smiles>CCN(CCCCCCO)c1cccc(C)c1</smiles>

Compound 20 was prepared as a colorless oil in 91\% yield (107.3 mg, eluent: petroleum ether/EtOAc = 10/1), from $(E)-N$-Ethyl-N-(hex-2-en-1-yl)-3-methylaniline 10 (108.6 mg, 0.50 $\mathrm{mmol}), \mathrm{Co}(\mathrm{acac}) 2(6.5 \mathrm{mg}, 0.025 \mathrm{mmol})$, tpy (5.9 mg, $0.025 \mathrm{mmol})$, and HBpin $(96.0 \mathrm{mg}, 0.75$ $\mathrm{mmol})$ following the above general procedure $\mathrm{H}$.

$\mathbf{R}_{f}=0.37$ (petroleum ether/EtOAc $=5 / 1$ )

${ }^{1} \mathrm{H}$ NMR (400 MHz, $\left.\mathrm{CDCl}_{3}\right) \delta 7.12-7.06(\mathrm{~m}, 1 \mathrm{H}), 6.50-6.45(\mathrm{~m}, 3 \mathrm{H}), 3.65(\mathrm{t}, J=6.6 \mathrm{~Hz}, 2 \mathrm{H})$, $3.33(\mathrm{t}, J=7.0 \mathrm{~Hz}, 2 \mathrm{H}), 3.26-3.21(\mathrm{~m}, 2 \mathrm{H}), 2.30(\mathrm{~s}, 3 \mathrm{H}), 1.62-1.55(\mathrm{~m}, 4 \mathrm{H}), 1.43-1.33(\mathrm{~m}, 4 \mathrm{H})$, $1.14(\mathrm{t}, J=7.0 \mathrm{~Hz}, 3 \mathrm{H})$.

${ }^{13} \mathrm{C}$ NMR (100 MHz, $\left.\mathrm{CDCl}_{3}\right) \delta$ 148.2, 139.0, 129.2, 116.4, 112.6, 109.2, 63.07, 50.5, 45.1, 32.9, 27.7, 27.2, 25.8, 22.2, 12.5 .

HRMS (ESI-TOF) calcd for $\mathrm{C}_{15} \mathrm{H}_{26} \mathrm{NO}^{+}[\mathrm{M}+\mathrm{H}]^{+}$: 236.2009, found: 236.2016.

IR (neat, cm-1): 3567, 2930, 1600, 1496, 1373, 1274, 1177, 1033, 762, 692. 


\section{6-(Butyl(phenyl)amino)hexan-1-ol (2p)}

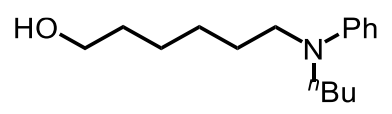

Compound $2 \mathrm{p}$ was prepared as a colorless oil in $76 \%$ yield $(94.8 \mathrm{mg}$, eluent: petroleum ether/EtOAc = 5/1), from (E)-N-butyl-N-(hex-2-en-1-yl)aniline 1p (115.6 mg, $0.50 \mathrm{mmol})$, Co(acac)2 $(6.5 \mathrm{mg}, 0.025 \mathrm{mmol})$, tpy $(5.9 \mathrm{mg}, 0.025 \mathrm{mmol})$, and HBpin $(96.0 \mathrm{mg}, 0.75 \mathrm{mmol})$ following the above general procedure $\mathrm{H}$.

$\mathbf{R}_{f}=0.30$ (petroleum ether/EtOAc $=5 / 1$ )

${ }^{1} \mathrm{H}$ NMR (400 MHz, $\left.\mathrm{CDCl}_{3}\right) \delta 7.23$ - $7.17(\mathrm{~m}, 2 \mathrm{H}), 6.67-6.61(\mathrm{~m}, 3 \mathrm{H}), 3.65(\mathrm{t}, J=6.6 \mathrm{~Hz}, 2 \mathrm{H})$, $3.28-3.23(\mathrm{~m}, 4 \mathrm{H}), 1.62-1.53(\mathrm{~m}, 6 \mathrm{H}), 1.41-1.32(\mathrm{~m}, 6 \mathrm{H}), 0.96(\mathrm{t}, J=7.3 \mathrm{~Hz}, 3 \mathrm{H})$.

${ }^{13} \mathrm{C}$ NMR (100 MHz, $\left.\mathrm{CDCl}_{3}\right) \delta$ 148.3, 129.3, 115.3, 111.8, 63.0, 51.1, 50.9, 32.9, 29.5, 27.4, 27.1, $25.8,20.5,14.2$.

HRMS (ESI-TOF) calcd for $\mathrm{C}_{16} \mathrm{H}_{28} \mathrm{NO}^{+}[\mathrm{M}+\mathrm{H}]^{+}:$250.2165, found: 250.2172 .

IR (neat, cm-1): 3398, 2930, 1600, 1496, 1373, 1274, 1033, 762, 692.

\section{6-(Isopropyl(phenyl)amino)hexan-1-ol (2q)}

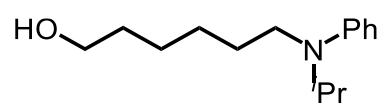

Compound $2 \mathbf{q}$ was prepared as a colorless oil in $50 \%$ yield (58.8 $\mathrm{mg}$, eluent: petroleum ether/EtOAc = 5/1), from $(E)-N$-(hex-2-en-1-yl)- $N$-isopropylaniline 1q $(108.6 \mathrm{mg}, 0.50 \mathrm{mmol})$, Co(acac)2 $(6.5 \mathrm{mg}, 0.025 \mathrm{mmol})$, tpy $(5.9 \mathrm{mg}, 0.025 \mathrm{mmol})$, and HBpin $(96.0 \mathrm{mg}, 0.75 \mathrm{mmol})$ following the above general procedure $\mathrm{H}$.

$\mathbf{R}_{f}=0.37$ (petroleum ether/EtOAc $=5 / 1$ )

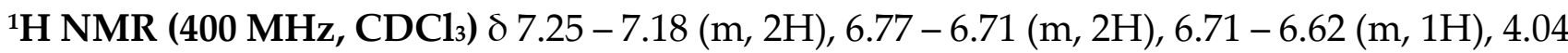
$(\mathrm{p}, J=6.6 \mathrm{~Hz}, 1 \mathrm{H}), 3.66(\mathrm{t}, J=6.6 \mathrm{~Hz}, 2 \mathrm{H}), 3.15-3.08(\mathrm{~m}, 2 \mathrm{H}), 1.63-1.54(\mathrm{~m}, 4 \mathrm{H}), 1.44-1.37$ $(\mathrm{m}, 4 \mathrm{H}), 1.18(\mathrm{~d}, J=6.6 \mathrm{~Hz}, 6 \mathrm{H})$.

${ }^{13} \mathrm{C}$ NMR (100 MHz, $\left.\mathrm{CDCl}_{3}\right) \delta$ 148.9, 129.3, 116.1, 113.4, 63.0, 48.7, 44.0, 32.9, 29.4, 27.2, 25.8, 20.2.

HRMS (ESI-TOF) calcd for $\mathrm{C}_{15} \mathrm{H}_{26} \mathrm{NO}^{+}[\mathrm{M}+\mathrm{H}]^{+}$: 236.2009, found: 236.2016.

IR (neat, cm-1): 3377, 1596, 1505, 1056, 745, 691.

\section{6-(Benzyl(phenyl)amino)hexan-1-ol (2r)}

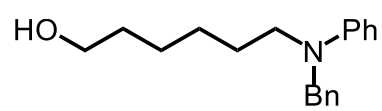


Compound $2 \mathrm{r}$ was prepared as a colorless oil in 77\% yield (108.6 mg, eluent: petroleum ether/EtOAc = 10/1), from $(E)-N$-benzyl-N-(hex-2-en-1-yl)aniline 1r (132.6 mg, $0.50 \mathrm{mmol})$, Co(acac)2 $(6.5 \mathrm{mg}, 0.025 \mathrm{mmol})$, tpy $(5.9 \mathrm{mg}, 0.025 \mathrm{mmol})$, and HBpin $(96.0 \mathrm{mg}, 0.75 \mathrm{mmol})$ following the above general procedure $\mathrm{H}$.

$\mathbf{R}_{f}=0.40$ (petroleum ether/EtOAc $=5 / 1$ )

${ }^{1}$ H NMR (400 MHz, $\mathrm{CDCl}_{3}$ ) $\delta 7.37$ - 7.30 (m, 2H), $7.28-7.18(\mathrm{~m}, 5 \mathrm{H}), 6.74-6.67$ (m, 3H), 4.57 $(\mathrm{s}, 2 \mathrm{H}), 3.67(\mathrm{t}, J=6.5 \mathrm{~Hz}, 2 \mathrm{H}), 3.46-3.39(\mathrm{~m}, 2 \mathrm{H}), 1.70(\mathrm{q}, J=7.3 \mathrm{~Hz}, 2 \mathrm{H}), 1.59$ (q, J = 6.9 Hz, 2H), $1.45-1.37(\mathrm{~m}, 4 \mathrm{H})$.

${ }^{13} \mathrm{C}$ NMR (100 MHz, $\left.\mathrm{CDCl}_{3}\right) \delta$ 148.7, 139.2, 129.3, 128.7, 126.8, 126.6, 116.1, 112.2, 63.0, 54.6, $51.3,32.7,27.2,27.1,25.8$.

HRMS (ESI-TOF) calcd for $\mathrm{C}_{19} \mathrm{H}_{26} \mathrm{NO}^{+}[\mathrm{M}+\mathrm{H}]^{+}: 284.2009$, found: 284.2018.

IR (neat, cm$^{-1}$ ): 3377, 2932, 1596, 1505, 745, 691.

\section{6-(Methyl( $p$-tolyl)amino)hexan-1-ol (2s)}

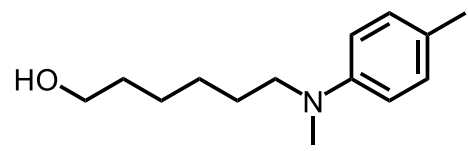

Compound 2s was prepared as a colorless oil in $79 \%$ yield $(87.4 \mathrm{mg}$, eluent: petroleum ether/EtOAc = 5/1), from $(E)-N$-(hex-2-en-1-yl)-N,4-dimethylaniline 1s (101.6 mg, $0.50 \mathrm{mmol})$, $\mathrm{Co}($ acac) $2(6.5 \mathrm{mg}, 0.025 \mathrm{mmol})$, tpy $(5.9 \mathrm{mg}, 0.025 \mathrm{mmol})$, and HBpin $(96.0 \mathrm{mg}, 0.75 \mathrm{mmol})$ following the above general procedure $\mathrm{H}$.

$\mathbf{R}_{f}=0.25$ (petroleum ether $/ \mathrm{EtOAc}=5 / 1$ )

${ }^{1}$ H NMR (400 MHz, $\left.\mathrm{CDCl}_{3}\right) \delta 7.03(\mathrm{~d}, J=8.5 \mathrm{~Hz}, 2 \mathrm{H}), 6.63(\mathrm{~d}, J=8.5 \mathrm{~Hz}, 2 \mathrm{H}), 3.64(\mathrm{t}, J=6.6$ $\mathrm{Hz}, 2 \mathrm{H}), 3.26(\mathrm{~m}, 2 \mathrm{H}), 2.88(\mathrm{~s}, 3 \mathrm{H}), 2.25(\mathrm{~s}, 3 \mathrm{H}), 1.60-1.54(\mathrm{~m}, 4 \mathrm{H}), 1.42-1.33(\mathrm{~m}, 4 \mathrm{H})$.

${ }^{13} \mathrm{C}$ NMR (100 MHz, $\left.\mathrm{CDCl}_{3}\right) \delta$ 147.6, 129.8, 125.4, 112.8, 63.1, 53.2, 38.6, 32.9, 27.2, 26.7, 25.82, 20.3 .

HRMS (ESI-TOF) calcd for $\mathrm{C}_{14} \mathrm{H}_{24} \mathrm{NO}^{+}[\mathrm{M}+\mathrm{H}]^{+}:$222.1852, found: 222.1862.

IR (neat, cm$\left.^{-1}\right)$ : 3377, 2932, 1596, 1505, 1056, 745, 691.

\section{6-(Methyl(m-tolyl)amino)hexan-1-ol (2t)}

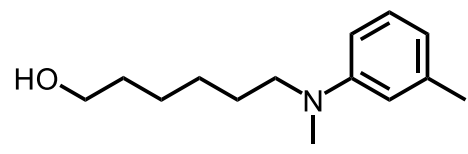

Compound $\mathbf{2 t}$ was prepared as a colorless oil in $84 \%$ yield $(92.9 \mathrm{mg}$, eluent: petroleum ether/EtOAc = 5/1), from $(E)-N$-(hex-2-en-1-yl)-N,3-dimethylaniline 1t (101.6 mg, 0.50 mmol), 
Co(acac)2 $(6.5 \mathrm{mg}, 0.025 \mathrm{mmol})$, tpy $(5.9 \mathrm{mg}, 0.025 \mathrm{mmol})$, and HBpin $(96.0 \mathrm{mg}, 0.75 \mathrm{mmol})$ following the above general procedure $\mathrm{H}$.

$\mathbf{R}_{f}=0.47$ (petroleum ether/EtOAc $=3 / 1$ )

${ }^{1}$ H NMR (400 MHz, $\left.\mathrm{CDCl}_{3}\right) \delta 7.13-7.08(\mathrm{~m}, 1 \mathrm{H}), 6.53-6.49(\mathrm{~m}, 3 \mathrm{H}), 3.64(\mathrm{t}, J=6.6 \mathrm{~Hz}, 2 \mathrm{H})$, $3.32-3.27(\mathrm{~m}, 2 \mathrm{H}), 2.91(\mathrm{~s}, 3 \mathrm{H}), 2.31(\mathrm{~s}, 3 \mathrm{H}), 1.61-1.55(\mathrm{~m}, 4 \mathrm{H}), 1.41-1.34(\mathrm{~m}, 4 \mathrm{H})$.

${ }^{13} \mathrm{C}$ NMR (100 MHz, $\left.\mathrm{CDCl}_{3}\right) \delta$ 149.6, 138.9, 129.1, 117.0, 113.0, 109.6, 63.0, 52.9, 38.5, 32.9, 27.1, $26.9,25.8,22.1$.

HRMS (ESI-TOF) calcd for $\mathrm{C}_{14} \mathrm{H}_{24} \mathrm{NO}^{+}[\mathrm{M}+\mathrm{H}]^{+}:$222.1852, found: 222.1862 .

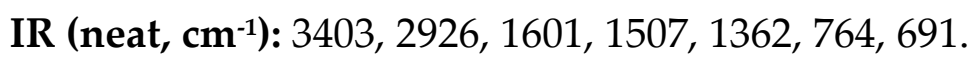

6-((4-Methoxyphenyl)(methyl)amino)hexan-1-ol (2u)<smiles>COc1ccc(N(C)CCCCCCO)cc1</smiles>

Compound $2 \mathbf{u}$ was prepared as a colorless oil in $62 \%$ yield $(73.9 \mathrm{mg}$, eluent: petroleum ether/EtOAc = 4/1), from $(E)-N$-(hex-2-en-1-yl)-4-methoxy- $N$-methylaniline 1u (109.6 mg, 0.50 $\mathrm{mmol}), \mathrm{Co}(\mathrm{acac})_{2}(6.5 \mathrm{mg}, 0.025 \mathrm{mmol})$, tpy (5.9 mg, $\left.0.025 \mathrm{mmol}\right)$, and HBpin (96.0 mg, 0.75 $\mathrm{mmol}$ ) following the above general procedure $\mathrm{H}$.

$\mathbf{R}_{f}=0.38$ (petroleum ether $/$ EtOAc $=1 / 1$ )

${ }^{1}$ H NMR (400 MHz, $\left.\mathrm{CDCl}_{3}\right) \delta 6.84-6.81(\mathrm{~m}, 2 \mathrm{H}), 6.71(\mathrm{~d}, J=9.0 \mathrm{~Hz}, 2 \mathrm{H}), 3.76(\mathrm{~s}, 3 \mathrm{H}), 3.63(\mathrm{t}$, $J=6.6 \mathrm{~Hz}, 2 \mathrm{H}), 3.23-3.19(\mathrm{~m}, 2 \mathrm{H}), 2.85(\mathrm{~s}, 3 \mathrm{H}), 1.59-1.52(\mathrm{~m}, 4 \mathrm{H}), 1.40-1.32(\mathrm{~m}, 4 \mathrm{H})$.

${ }^{13} \mathrm{C}$ NMR (100 MHz, $\left.\mathrm{CDCl}_{3}\right) \delta 151.6,144.6,114.9,114.7,62.9,55.9,54.1,39.1,32.8,27.1,26.6$, 25.8 .

HRMS (ESI-TOF) calcd for $\mathrm{C}_{14} \mathrm{H}_{24} \mathrm{NO}_{2}{ }^{+}[\mathrm{M}+\mathrm{H}]^{+}:$238.1802, found: 238.1808.

IR (neat, cm$^{-1}$ ): 3403, 2926, 2857, 1601, 1507, 1362, 764, 691.

6-((4-Fluorophenyl)(methyl)amino)hexan-1-ol (2v)

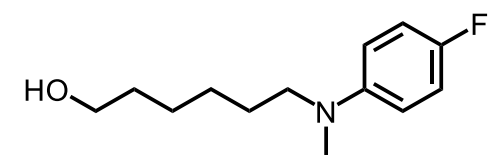

Compound $2 \mathbf{v}$ was prepared as a colorless oil in $79 \%$ yield $(88.4 \mathrm{mg}$, eluent: petroleum ether/EtOAc = 5/1), from $(E)$-4-fluoro- $N$-(hex-2-en-1-yl)- $N$-methylaniline 1v (103.6 mg, 0.50 $\mathrm{mmol}), \mathrm{Co}(\mathrm{acac}) 2(6.5 \mathrm{mg}, 0.025 \mathrm{mmol})$, tpy (5.9 mg, $0.025 \mathrm{mmol})$, and HBpin $(96.0 \mathrm{mg}, 0.75$ $\mathrm{mmol})$ following the above general procedure $\mathrm{H}$.

$\mathbf{R}_{f}=0.30$ (petroleum ether/EtOAc $=5 / 1$ ) 
${ }^{1} \mathrm{H}$ NMR (400 MHz, $\left.\mathrm{CDCl}_{3}\right) \delta 6.96-6.89(\mathrm{~m}, 2 \mathrm{H}), 6.64-6.60(\mathrm{~m}, 2 \mathrm{H}), 3.64(\mathrm{t}, J=6.6 \mathrm{~Hz}, 2 \mathrm{H})$, $3.27-3.22(\mathrm{~m}, 2 \mathrm{H}), 2.87(\mathrm{~s}, 3 \mathrm{H}), 1.60-1.53(\mathrm{~m}, 4 \mathrm{H}), 1.42-1.34(\mathrm{~m}, 4 \mathrm{H})$.

${ }^{13} \mathrm{C}$ NMR (100 MHz, $\left.\mathrm{CDCl}_{3}\right) \delta 155.3$ (d, J = $\left.233 \mathrm{~Hz}\right), 146.4,115.6$ (d, J = $\left.22 \mathrm{~Hz}\right), 113.6$ (d, J = 8 $\mathrm{Hz}), 63.0,53.7,38.9,32.9,27.1,26.6,25.8$.

${ }^{19} \mathrm{~F}$ NMR (377 MHz, $\left.\mathrm{CDCl}_{3}\right) \delta-129.8(\mathrm{~s})$.

HRMS (ESI-TOF) calcd for $\mathrm{C}_{13} \mathrm{H}_{21} \mathrm{FNO}^{+}[\mathrm{M}+\mathrm{H}]^{+}: 226.1602$, found: 226.1611 .

IR (neat, cm$\left.^{-1}\right)$ : 3567, 2930, 2860, 1507, 1225, 810.

\section{6-((4-Chlorophenyl)(methyl)amino)hexan-1-ol (2w)}

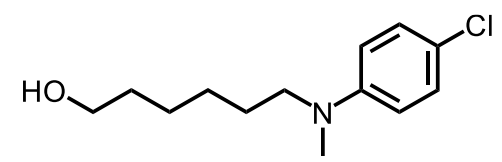

Compound $2 \mathbf{w}$ was prepared as a colorless oil in $35 \%$ yield $(42.4 \mathrm{mg}$, eluent: petroleum ether/EtOAc = 5/1), from $(E)$-4-chloro- $N$-(hex-2-en-1-yl)-N-methylaniline 1w (115.6 mg, 0.50 $\mathrm{mmol}), \mathrm{Co}(\mathrm{acac}) 2(6.5 \mathrm{mg}, 0.025 \mathrm{mmol})$, tpy (5.9 mg, $0.025 \mathrm{mmol})$, and HBpin $(96.0 \mathrm{mg}, 0.75$ mmol) following the above general procedure $\mathrm{H}$.

$\mathbf{R}_{f}=0.20$ (petroleum ether $/ \mathrm{EtOAc}=5 / 1$ )

${ }^{1}$ H NMR (400 MHz, $\left.\mathrm{CDCl}_{3}\right) \delta 7.14(\mathrm{~d}, J=9.0 \mathrm{~Hz}, 2 \mathrm{H}), 6.59(\mathrm{~d}, J=8.9 \mathrm{~Hz}, 2 \mathrm{H}), 3.64(\mathrm{t}, J=6.5$ $\mathrm{Hz}, 2 \mathrm{H}), 3.29-3.25(\mathrm{~m}, 2 \mathrm{H}), 2.89(\mathrm{~s}, 3 \mathrm{H}), 1.59-1.53(\mathrm{~m}, 4 \mathrm{H}), 1.42-1.33(\mathrm{~m}, 4 \mathrm{H})$.

${ }^{13} \mathrm{C}$ NMR (100 MHz, $\left.\mathrm{CDCl}_{3}\right) \delta$ 148.1, 129.0, 120.8, 113.3, 63.0, 53.0, 38.6, 32.9, 27.1, 26.7, 25.8.

HRMS (ESI-TOF) calcd for $\mathrm{C}_{13} \mathrm{H}_{21} \mathrm{ClNO}^{+}[\mathrm{M}+\mathrm{H}]^{+}: 242.1306$, found: 242.1319.

IR (neat, cm ${ }^{-1}$ ): 3378, 2930, 2860, 1507, 1457, 1225, 810.

Methyl 4-((6-hydroxyhexyl)(methyl)amino)benzoate (2x)<smiles>CC(=O)c1ccc(N(C)CCCCCCO)cc1</smiles>

Compound $2 \mathrm{x}$ was prepared as a colorless oil in $64 \%$ yield $(85.8 \mathrm{mg}$, eluent: petroleum ether/EtOAc = 5/1), from methyl $(E)$-4-(hex-2-en-1-yl(methyl)amino)benzoate 1x (123.6 mg, $0.50 \mathrm{mmol}), \mathrm{Co}(\mathrm{acac}) 2(6.5 \mathrm{mg}, 0.025 \mathrm{mmol})$, tpy $(5.9 \mathrm{mg}, 0.025 \mathrm{mmol})$, and HBpin $(96.0 \mathrm{mg}$, $0.75 \mathrm{mmol}$ ) following the above general procedure J.

$\mathbf{R}_{f}=0.32$ (petroleum ether $/$ EtOAc $=5 / 1$ )

${ }^{1}$ H NMR (400 MHz, $\left.\mathrm{CDCl}_{3}\right) \delta 7.88(\mathrm{~d}, J=9.0 \mathrm{~Hz}, 2 \mathrm{H}), 6.61(\mathrm{~d}, J=9.0 \mathrm{~Hz}, 2 \mathrm{H}), 3.84(\mathrm{~s}, 3 \mathrm{H}), 3.64$ $(\mathrm{t}, J=6.5 \mathrm{~Hz}, 2 \mathrm{H}), 3.39-3.34(\mathrm{~m}, 2 \mathrm{H}), 2.99(\mathrm{~s}, 3 \mathrm{H}), 1.63-1.55(\mathrm{~m}, 4 \mathrm{H}), 1.42-1.34(\mathrm{~m}, 4 \mathrm{H})$.

${ }^{13} \mathrm{C}$ NMR (100 MHz, $\left.\mathrm{CDCl}_{3}\right) \delta$ 167.6, 152.4, 131.5, 116.6, 110.6, 62.9, 52.4, 51.6, 38.5, 32.8, 27.00, 
$26.95,25.8$.

HRMS (ESI-TOF) calcd for $\mathrm{C}_{15} \mathrm{H}_{24} \mathrm{NO}_{3}{ }^{+}[\mathrm{M}+\mathrm{H}]^{+}:$266.1751, found: 266.1760 .

IR (neat, cm-1): 3412, 2930, 1700, 1602, 1525, 1278, 1180, 1109, 769.

6-(3,4-Dihydroquinolin-1(2H)-yl)hexan-1-ol (2y)

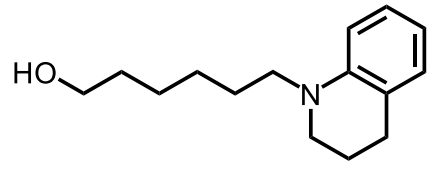

Compound $2 \mathbf{y}$ was prepared as a colorless oil in $32 \%$ yield $(37.1 \mathrm{mg}$, eluent: petroleum ether/EtOAc = 5/1), from $(E)$-1-(hex-2-en-1-yl)-1,2,3,4-tetrahydroquinoline 1y (116.6 mg, 0.50 $\mathrm{mmol}), \mathrm{Co}(\mathrm{acac})_{2}(6.5 \mathrm{mg}, 0.025 \mathrm{mmol})$, tpy (5.9 mg, $\left.0.025 \mathrm{mmol}\right)$, and HBpin (96.0 mg, 0.75 $\mathrm{mmol}$ ) following the above general procedure $\mathrm{H}$.

$\mathbf{R}_{f}=0.37$ (petroleum ether/EtOAc $=5 / 1$ )

${ }^{1}$ H NMR (400 MHz, $\left.\mathrm{CDCl}_{3}\right) \delta 7.06-7.01(\mathrm{~m}, 1 \mathrm{H}), 6.96-6.91(\mathrm{~m}, 1 \mathrm{H}), 6.54(\mathrm{t}, J=7.7 \mathrm{~Hz}, 2 \mathrm{H})$, $3.65(\mathrm{t}, J=6.5 \mathrm{~Hz}, 2 \mathrm{H}), 3.28-3.20(\mathrm{~m}, 4 \mathrm{H}), 2.75(\mathrm{t}, J=6.4 \mathrm{~Hz}, 2 \mathrm{H}), 1.98-1.91(\mathrm{~m}, 2 \mathrm{H}), 1.63-$ $1.57(\mathrm{~m}, 4 \mathrm{H}), 1.42-1.32(\mathrm{~m}, 4 \mathrm{H})$.

${ }^{13} \mathrm{C}$ NMR (100 MHz, $\left.\mathrm{CDCl}_{3}\right) \delta$ 145.4, 129.3, 127.2, 122.3, 115.3, 110.5, 63.1, 51.6, 49.6, 32.9, 28.3, $27.2,26.3,25.8,22.4$.

The ${ }^{1} \mathrm{H}$ and ${ }^{13} \mathrm{C}$ NMR spectroscopic data match the reported characterization data. ${ }^{28}$

\section{6-(Diphenylamino)hexan-1-ol (2z)}<smiles>OCCCCCCN(c1ccccc1)c1ccccc1</smiles>

Compound $\mathbf{2 z}$ was prepared as a colorless oil in $64 \%$ yield $(86.1 \mathrm{mg}$, eluent: petroleum ether/EtOAc = 5/1), from $(E)-N$-(hex-2-en-1-yl)- $N$-phenylaniline $1 z$ (134.6 mg, $0.50 \mathrm{mmol})$, Co(acac)2 $(6.5 \mathrm{mg}, 0.025 \mathrm{mmol})$, tpy $(5.9 \mathrm{mg}, 0.025 \mathrm{mmol})$, and HBpin $(96.0 \mathrm{mg}, 0.75 \mathrm{mmol})$ following the above general procedure $\mathrm{H}$.

$\mathbf{R}_{f}=0.38$ (petroleum ether $/$ EtOAc $=5 / 1$ )

${ }^{1}$ H NMR (400 MHz, $\left.\mathrm{CDCl}_{3}\right) \delta 7.33$ - $7.28(\mathrm{~m}, 4 \mathrm{H}), 7.05-6.96(\mathrm{~m}, 6 \mathrm{H}), 3.75-3.71(\mathrm{~m}, 2 \mathrm{H}), 3.65$ $(\mathrm{t}, J=6.6 \mathrm{~Hz}, 2 \mathrm{H}), 1.76-1.67(\mathrm{~m}, 2 \mathrm{H}), 1.62-1.56(\mathrm{~m}, 2 \mathrm{H}), 1.44-1.37(\mathrm{~m}, 4 \mathrm{H})$.

${ }^{13} \mathrm{C}$ NMR (100 MHz, $\left.\mathrm{CDCl}_{3}\right) \delta$ 148.2, 129.4, 121.2, 121.00, 62.9, 52.3, 32.8, 27.6, 27.0, 25.7.

The ${ }^{1} \mathrm{H}$ and ${ }^{13} \mathrm{C}$ NMR spectroscopic data match the reported characterization data. ${ }^{29}$

6-(9H-Carbazol-9-yl)hexan-1-ol (2aa) 


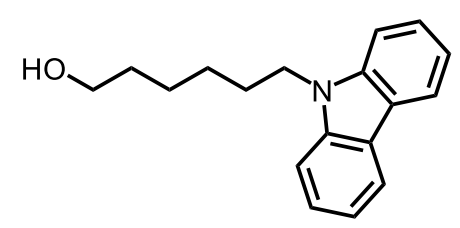

Compound 2aa was prepared as a white solid in $26 \%$ yield (33.5 mg, eluent: petroleum ether/EtOAc = 5/1), from (E)-9-(hex-2-en-1-yl)-9H-carbazole 1aa (124.6 mg, $0.50 \mathrm{mmol})$, Co(acac)2 $(6.5 \mathrm{mg}, 0.025 \mathrm{mmol})$, tpy $(5.9 \mathrm{mg}, 0.025 \mathrm{mmol})$, and HBpin $(96.0 \mathrm{mg}, 0.75 \mathrm{mmol})$ following the above general procedure $\mathrm{H}$.

$\mathbf{R}_{f}=0.25$ (petroleum ether/EtOAc $=5 / 1$ )

${ }^{1} \mathrm{H}$ NMR (400 MHz, $\left.\mathrm{CDCl}_{3}\right) \delta 8.14(\mathrm{~d}, J=7.8 \mathrm{~Hz}, 2 \mathrm{H}), 7.51-7.42(\mathrm{~m}, 4 \mathrm{H}), 7.26$ (t, J = 7.3 Hz, $2 \mathrm{H}), 4.34(\mathrm{t}, J=7.2 \mathrm{~Hz}, 2 \mathrm{H}), 3.62(\mathrm{t}, J=6.5 \mathrm{~Hz}, 2 \mathrm{H}), 1.96-1.89(\mathrm{~m}, 2 \mathrm{H}), 1.55-1.40(\mathrm{~m}, 6 \mathrm{H})$.

${ }^{13} \mathrm{C}$ NMR (100 MHz, $\left.\mathrm{CDCl}_{3}\right) \delta$ 140.5, 125.7, 123.0, 120.5, 118.9, 108.8, 62.91, 43.1, 32.7, 29.1, 27.2, 25.7 .

The ${ }^{1} \mathrm{H}$ and ${ }^{13} \mathrm{C}$ NMR spectroscopic data match the reported characterization data. ${ }^{30}$

\section{1-(6-Hydroxyhexyl)pyrrolidin-2-one (2ab)}

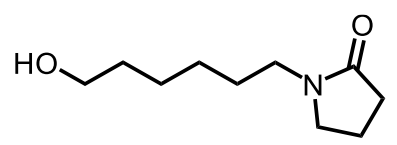

Compound $2 \mathrm{ab}$ was prepared as a colorless oil in $53 \%$ yield $(48.9 \mathrm{mg}$, eluent: $\mathrm{EtOAc} / \mathrm{MeOH}$ = 20/1), from (E)-9-(hex-2-en-1-yl)-9H-carbazole $1 \mathrm{ab}(83.6 \mathrm{mg}, 0.50 \mathrm{mmol}), \mathrm{Co}(\mathrm{acac}) 2$ (6.5 mg, $0.025 \mathrm{mmol})$, tpy (5.9 mg, $0.025 \mathrm{mmol})$, and HBpin $(96.0 \mathrm{mg}, 0.75 \mathrm{mmol})$ following the above general procedure $\mathrm{H}$.

$\mathbf{R}_{f}=0.39(\mathrm{EtOAc} / \mathrm{MeOH}=10 / 1)$

${ }^{1} \mathrm{H}$ NMR (400 MHz, $\left.\mathrm{CDCl}_{3}\right) \delta 3.62(\mathrm{t}, J=6.4 \mathrm{~Hz}, 2 \mathrm{H}), 3.36(\mathrm{t}, J=7.1 \mathrm{~Hz}, 2 \mathrm{H}), 3.27(\mathrm{t}, J=7.3 \mathrm{~Hz}$, 2H), $2.37(\mathrm{t}, J=8.1 \mathrm{~Hz}, 2 \mathrm{H}), 2.04-1.97(\mathrm{~m}, 2 \mathrm{H}), 1.58-1.49(\mathrm{~m}, 4 \mathrm{H}), 1.43-1.36(\mathrm{~m}, 2 \mathrm{H}), 1.33-$ $1.27(\mathrm{~m}, 2 \mathrm{H})$.

${ }^{13} \mathrm{C}$ NMR (100 MHz, $\left.\mathrm{CDCl}_{3}\right) \delta$ 175.2, 62.7, 47.2, 42.4, 32.7, 31.3, 27.3, 26.4, 25.3, 18.1.

HRMS (ESI-TOF) calcd for $\mathrm{C}_{10} \mathrm{H}_{20} \mathrm{NO}_{2}{ }^{+}[\mathrm{M}+\mathrm{H}]^{+}:$186.1489, found: 186.1498.

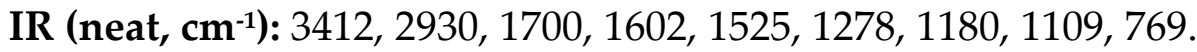

\section{tert-Butyl benzyl(6-hydroxyhexyl)carbamate (2ac)}

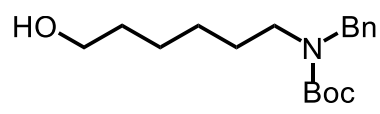

Compound 2ac was prepared as a colorless oil in $80 \%$ yield $(122.9 \mathrm{mg}$, eluent: petroleum ether/EtOAc $=5 / 1)$, from tert-butyl $(E)$-benzyl(hex-2-en-1-yl)carbamate 1ac $(144.6 \mathrm{mg}, 0.50$ 
$\mathrm{mmol}), \mathrm{Co}($ acac)2 $(6.5 \mathrm{mg}, 0.025 \mathrm{mmol})$, tpy (5.9 mg, $0.025 \mathrm{mmol})$, and HBpin $(96.0 \mathrm{mg}, 0.75$ $\mathrm{mmol}$ ) following the above general procedure $\mathrm{H}$.

$\mathbf{R}_{f}=0.21$ (petroleum ether $/ \mathrm{EtOAc}=5 / 1$ )

${ }^{1} \mathrm{H}$ NMR (400 MHz, $\left.\mathrm{CDCl}_{3}\right) \delta 7.34-7.27(\mathrm{~m}, 2 \mathrm{H}), 7.25-7.19(\mathrm{~m}, 3 \mathrm{H}), 4.41(\mathrm{~s}, 2 \mathrm{H}), 3.60(\mathrm{t}, J=$ $6.5 \mathrm{~Hz}, 2 \mathrm{H}), 3.17(\mathrm{~s}, 2 \mathrm{H}), 1.56-1.32(\mathrm{~m}, 17 \mathrm{H})$.

${ }^{13} \mathrm{C}$ NMR (100 MHz, $\left.\mathrm{CDCl}_{3}\right) \delta$ 156.2, 138.9, 128.6, 127.6, 127.2, 79.7, 62.9, 50.5, 46.7, 32.9, 28.6, $28.2,26.7,25.5$.

The ${ }^{1} \mathrm{H}$ and ${ }^{13} \mathrm{C}$ NMR spectroscopic data match the reported characterization data. ${ }^{31}$

\section{$N$-Benzyl-N-(6-hydroxyhexyl)pivalamide (2ad)}

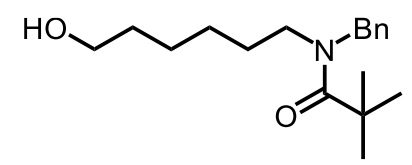

Compound 2ad was prepared as a colorless oil in $88 \%$ yield (128.1 mg, eluent: petroleum ether/EtOAc = 5/1), from tert-butyl $(E)-N$-benzyl- $N$-(hex-2-en-1-yl)pivalamide 1ad (136.6 mg, $0.50 \mathrm{mmol}), \mathrm{Co}($ acac $) 2(6.5 \mathrm{mg}, 0.025 \mathrm{mmol})$, tpy (5.9 mg, $0.025 \mathrm{mmol})$, and HBpin $(96.0 \mathrm{mg}$, $0.75 \mathrm{mmol}$ ) following the above general procedure $\mathrm{H}$.

$\mathbf{R}_{f}=0.15$ (petroleum ether $/$ EtOAc $=5 / 1$ )

${ }^{1} \mathrm{H}$ NMR (400 MHz, $\left.\mathrm{CDCl}_{3}\right) \delta 7.34-7.29(\mathrm{~m}, 2 \mathrm{H}), 7.26-7.24(\mathrm{~m}, 1 \mathrm{H}), 7.19-7.14(\mathrm{~m}, 2 \mathrm{H}), 4.67$ $(\mathrm{s}, 2 \mathrm{H}), 3.60(\mathrm{t}, J=6.4 \mathrm{~Hz}, 2 \mathrm{H}), 3.30-3.24(\mathrm{~m}, 2 \mathrm{H}), 1.58-1.51(\mathrm{~m}, 4 \mathrm{H}), 1.35-1.25(\mathrm{~m}, 13 \mathrm{H})$.

${ }^{13} \mathrm{C}$ NMR (100 MHz, $\left.\mathrm{CDCl}_{3}\right) \delta$ 177.9, 138.0, 128.8, 127.3, 127.2, 62.8, 50.6, 47.2, 39.4, 32.8, 28.9, 27.7, 26.8, 25.6.

HRMS (ESI-TOF) calcd for $\mathrm{C}_{18} \mathrm{H}_{30} \mathrm{NO}_{2}{ }^{+}[\mathrm{M}+\mathrm{H}]^{+}:$292.2271, found: 292.2279.

IR (neat, cm-1): 3385, 2934, 1608, 1418, 1181, 732, 697.

\section{Compound 2ae}

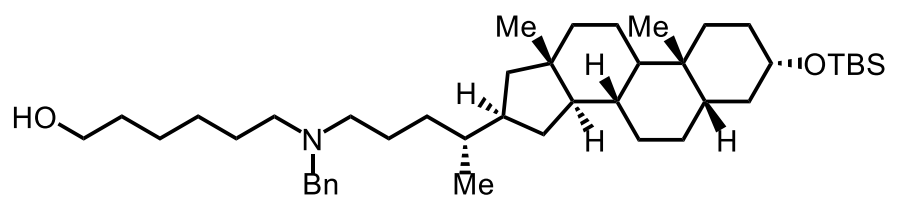

Compound 2ae was prepared as a colorless oil in 59\% yield (118.0 mg, eluent: petroleum ether/EtOAc $=5 / 1)$, from compound 1ae $(194.1 \mathrm{mg}, 0.30 \mathrm{mmol}), \mathrm{Co}(\mathrm{acac})_{2}(3.9 \mathrm{mg}, 0.015$ $\mathrm{mmol})$, tpy (3.5 mg, $0.015 \mathrm{mmol})$, and HBpin $(57.6 \mathrm{mg}, 0.45 \mathrm{mmol})$ following the above general procedure $\mathrm{H}$. 
$\mathbf{R}_{f}=0.25$ (petroleum ether $/$ EtOAc $=5 / 1$ )

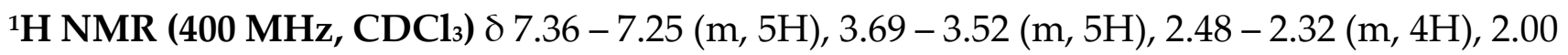
- $1.92(\mathrm{~m}, 1 \mathrm{H}), 1.86-1.78(\mathrm{~m}, 3 \mathrm{H}), 1.57-1.16(\mathrm{~m}, 32 \mathrm{H}), 0.93-0.90(\mathrm{~m}, 15 \mathrm{H}), 0.64(\mathrm{~s}, 3 \mathrm{H}), 0.08$ (s, 6H).

${ }^{13} \mathrm{C}$ NMR (100 MHz, $\left.\mathrm{CDCl}_{3}\right) \delta$ 140.1, 129.1, 128.2, 126.8, 73.0, 63.1, 58.8, 56.6, 56.4, 54.5, 53.8, $42.8,42.5,40.4,40.3,37.1,36.0,35.9,35.7,34.7,33.8,32.9,31.2,29.9,28.5,27.5,27.3,27.1,26.6$, $26.1,25.8,24.4,23.6,21.0,18.8,18.5,12.2,-4.4$.

HRMS (ESI-TOF) calcd for $\mathrm{C}_{43} \mathrm{H}_{76} \mathrm{NO}_{2} \mathrm{Si}^{+}[\mathrm{M}+\mathrm{H}]^{+}:$666.5640, found: 666.5648 .

IR (neat, cm-1): 3649, 2926, 1684, 1653, 1558, 1507, 1457, 1078, 835, 773, 738.

Compound 2af

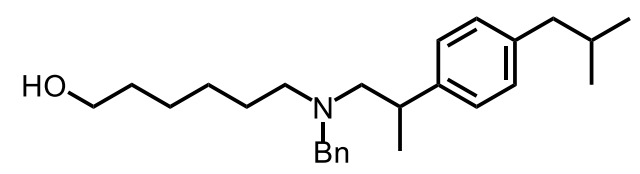

Compound 2af was prepared as a colorless oil in 63\% yield (119.8 mg, eluent: petroleum ether/EtOAc = 5/1), from compound 1af (181.5 mg, $0.50 \mathrm{mmol}), \mathrm{Co}(\mathrm{acac})_{2}(6.5 \mathrm{mg}, 0.025$ mmol), tpy (5.9 mg, $0.025 \mathrm{mmol})$, and HBpin $(96.0 \mathrm{mg}, 0.75 \mathrm{mmol}$ ) following the above general procedure $\mathrm{H}$.

$\mathbf{R}_{f}=0.20$ (petroleum ether/EtOAc $=5 / 1$ )

${ }^{1}$ H NMR (400 MHz, $\left.\mathbf{C D C l}_{3}\right) \delta 7.30-7.23(\mathrm{~m}, 5 \mathrm{H}), 7.08(\mathrm{~d}, J=0.9 \mathrm{~Hz}, 4 \mathrm{H}), 3.68-3.59(\mathrm{~m}, 3 \mathrm{H})$, $3.53-3.48(\mathrm{~m}, 1 \mathrm{H}), 2.99-2.87(\mathrm{~m}, 1 \mathrm{H}), 2.58-2.36(\mathrm{~m}, 6 \mathrm{H}), 1.92-1.84(\mathrm{~m}, 1 \mathrm{H}), 1.57-1.50(\mathrm{~m}$, $2 \mathrm{H}), 1.48-1.41(\mathrm{~m}, 2 \mathrm{H}), 1.31-1.23(\mathrm{~m}, 7 \mathrm{H}), 0.94(\mathrm{~d}, J=6.1 \mathrm{~Hz}, 6 \mathrm{H})$.

${ }^{13} \mathrm{C}$ NMR (100 MHz, $\left.\mathrm{CDCl}_{3}\right) \delta$ 143.6, 140.4, 139.3, 129.0, 128.9, 128.1, 127.1, 126.7, 63.1, 62.3, 59.3, 54.1, 45.2, 38.1, 32.9, 30.4, 27.2, 27.0, 25.7, 22.5, 20.1.

HRMS (ESI-TOF) calcd for $\mathrm{C}_{26} \mathrm{H}_{40} \mathrm{NO}^{+}[\mathrm{M}+\mathrm{H}]^{+}: 382.3140$, found: 382.3136 .

IR (neat, cm-1): 3649, 2926, 2857, 1558, 1507, 1457, 1374, 1078, 835.

\section{Compound 2ag}

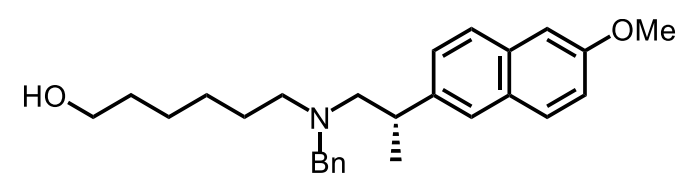

Compound 2ag was prepared as a colorless oil in 58\% yield (114.5 mg, eluent: petroleum ether/EtOAc = 5/1), from compound $1 \mathrm{ag}(193.5 \mathrm{mg}, 0.50 \mathrm{mmol})$, Co(acac)2 (6.5 mg, 0.025 $\mathrm{mmol})$, tpy $(5.9 \mathrm{mg}, 0.025 \mathrm{mmol})$, and HBpin $(96.0 \mathrm{mg}, 0.75 \mathrm{mmol})$ following the above 
general procedure $\mathrm{H}$.

$\mathbf{R}_{f}=0.15$ (petroleum ether $/ \mathrm{EtOAc}=5 / 1$ )

${ }^{1} \mathrm{H}$ NMR $\left(400 \mathrm{MHz}, \mathrm{CDCl}_{3}\right) \delta 7.70(\mathrm{~d}, J=8.8 \mathrm{~Hz}, 2 \mathrm{H}), 7.54(\mathrm{~s}, 1 \mathrm{H}), 7.32-7.23(\mathrm{~m}, 6 \mathrm{H}), 7.18-$ $7.10(\mathrm{~m}, 2 \mathrm{H}), 3.95(\mathrm{~s}, 3 \mathrm{H}), 3.70-3.52(\mathrm{~m}, 4 \mathrm{H}), 3.16-3.05(\mathrm{~m}, 1 \mathrm{H}), 2.74-2.65(\mathrm{~m}, 1 \mathrm{H}), 2.65-$ $2.54(\mathrm{~m}, 1 \mathrm{H}), 2.49-2.36(\mathrm{~m}, 2 \mathrm{H}), 1.52-1.39(\mathrm{~m}, 4 \mathrm{H}), 1.35(\mathrm{~d}, J=7.1,1.6 \mathrm{~Hz}, 3 \mathrm{H}), 1.28-1.14$ $(\mathrm{m}, 4 \mathrm{H})$.

${ }^{13} \mathrm{C}$ NMR (100 MHz, $\left.\mathrm{CDCl}_{3}\right) \delta$ 157.2, 141.7, 140.3, 133.3, 129.2, 129.01, 128.97, 128.1, 126.9, 126.7, 126.6, 125.5, 118.6, 105.7, 63.1, 62.2, 59.4, 55.4, 54.2, 38.4, 32.9, 27.2, 27.1, 25.7, 20.2.

HRMS (ESI-TOF) calcd for $\mathrm{C}_{27} \mathrm{H}_{36} \mathrm{NO}_{2}{ }^{+}[\mathrm{M}+\mathrm{H}]^{+}:$: 406.2741, found: 406.2743 .

IR (neat, cm ${ }^{-1}$ ): 3649, 2930, 1558, 1507, 1457, 737, 698.

\section{Gram-scale Reaction and Applications}

\section{Gram-scale Reaction}
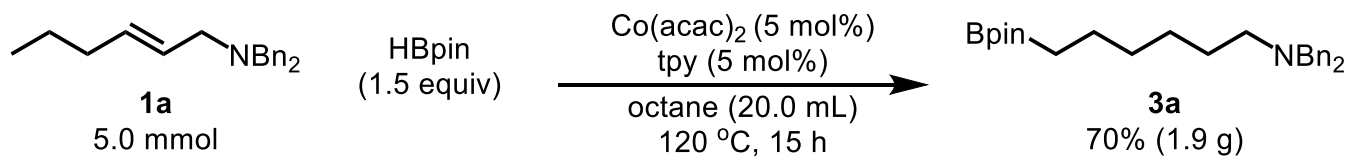

In a $\mathrm{N}_{2}$-filled glove box, to an oven-dried 100-mL sealed tube $\mathrm{Co}(\mathrm{acac}) 2(64.3 \mathrm{mg}, 0.25$ $\mathrm{mmol})$, tpy (58.4 mg, $0.25 \mathrm{mmol})$ and anhydrous octane $(20.0 \mathrm{~mL})$ were added. The resulting solution was stirred for $20 \mathrm{~min}$ at room temperature, at which time HBpin (1.0 g, $7.50 \mathrm{mmol})$ was added. After the resulting mixture was stirred for an additional $5 \mathrm{~min},(E)-\mathrm{N}, \mathrm{N}$ dibenzylhex-2-en-1-amine $1 \mathrm{a}(1.4 \mathrm{~g}, 5.00 \mathrm{mmol})$ was added and stirred for $15 \mathrm{~h}$ at $120{ }^{\circ} \mathrm{C}$ in an oil bath. The resulting suspension was cooled to room temperature, filtered through a celite pad with EtOAc and concentrated under vacuo. The residue was purified by silica gel chromatography to afford compound $3 \mathbf{a}$ as a colorless oil in $70 \%$ yield (1.9 $\mathrm{g}$, petroleum ether/EtOAc $=40 / 1)$.

$\mathbf{R}_{f}=0.36$ (petroleum ether $/$ EtOAc $=10 / 1$ )

${ }^{1} \mathrm{H}$ NMR (400 MHz, $\left.\mathrm{CDCl}_{3}\right) \delta 7.40-7.35(\mathrm{~m}, 4 \mathrm{H}), 7.34-7.28(\mathrm{~m}, 4 \mathrm{H}), 7.25$ - $7.18(\mathrm{~m}, 2 \mathrm{H}), 3.55$ $(\mathrm{s}, 4 \mathrm{H}), 2.41(\mathrm{t}, J=7.3 \mathrm{~Hz}, 2 \mathrm{H}), 1.56-1.47(\mathrm{~m}, 2 \mathrm{H}), 1.42-1.35(\mathrm{~m}, 2 \mathrm{H}), 1.29-1.22(\mathrm{~m}, 16 \mathrm{H})$, $0.76(\mathrm{t}, J=7.8 \mathrm{~Hz}, 2 \mathrm{H})$.

${ }^{13} \mathrm{C}$ NMR (100 MHz, $\mathrm{CDCl}_{3}$ ) $\delta$ 140.2, 128.8, 128.2, 126.8, 83.0, 58.4, 53.6, 32.5, 27.2, 27.1, 25.0, 24.1.

${ }^{11 B}$ NMR (128 MHz, $\left.\mathrm{CDCl}_{3}\right) \delta 34.1$.

The carbon signal attached to $\mathrm{B}$ was not observed due to low intensity.

HRMS (ESI-TOF) calcd for $\mathrm{C}_{26} \mathrm{H}_{39} \mathrm{BNO}_{2}{ }^{+}[\mathrm{M}+\mathrm{H}]^{+}: 408.3068$, found: 408.3072 . 
IR (neat, cm$\left.^{-1}\right)$ : 2926, 2857, 1684, 1558, 1507, 1457, 1078, 835, 773, 698.

\section{Synthesis of 4}

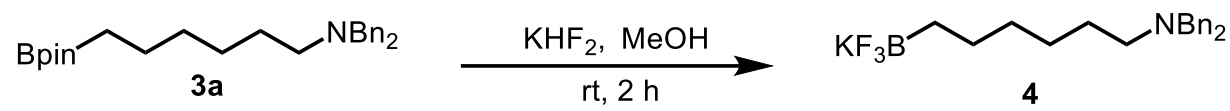

Following a modified procedure ${ }^{32}$, to a solution of $3 \mathbf{a}(84.1 \mathrm{mg}, 0.20 \mathrm{mmol})$ in $\mathrm{MeOH}$ $(0.50 \mathrm{~mL})$ was added $\mathrm{KHF}_{2}(0.25 \mathrm{~mL}$ of saturated aqueous solution $(4.0-4.5 \mathrm{M}), 2.00 \mathrm{mmol})$ dropwise at room temperature. The solution was stirred at room temperature for $2 \mathrm{~h}$, and then concentrated in vacuo. To the residue was added $60 \%$ aqueous $\mathrm{MeOH}$, which was then concentrated in vacuo. This procedure was repeated twice. The resulting white solid was dissolved in hot acetone, and ether was then added. The precipitate was washed with ether, and the resultant precipitate was dissolved in acetone, and concentrated in vacuo overnight to afford potassium trifluoroborate $4(59.0 \mathrm{mg}, 76 \%$ yield) as a white solid.

${ }^{1}$ H NMR (400 MHz, Acetone) $\delta 7.50$ - $7.40(\mathrm{~m}, 4 \mathrm{H}), 7.37-7.30(\mathrm{~m}, 4 \mathrm{H}), 7.29-7.21$ (m, 2H), $3.65(\mathrm{~s}, 4 \mathrm{H}), 2.48(\mathrm{t}, J=7.5 \mathrm{~Hz}, 2 \mathrm{H}), 1.62-1.50(\mathrm{~m}, 2 \mathrm{H}), 1.28-1.19(\mathrm{~m}, 6 \mathrm{H}), 0.24--0.04(\mathrm{~m}$, $2 \mathrm{H})$.

${ }^{13} \mathrm{C}$ NMR (100 MHz, Acetone) $\delta$ 140.3, 128.8, 128.3, 126.8, 58.2, 53.7, 33.8, 27.3, 25.7, 25.6.

${ }^{11} \mathrm{~B}$ NMR (128 MHz, $\left.\mathrm{CDCl}_{3}\right) \delta 5.8$.

The carbon signal attached to B was not observed due to low intensity.

HRMS (ESI-Orbitrap) calcd for $\mathrm{C}_{20} \mathrm{H}_{26} \mathrm{~N}^{10} \mathrm{BF}_{3}$ [M]: 347.2152, found: 347.2154.

IR (neat, cm-1): 2921, 1558, 1507, 1457, 1073, 907, 742, 696.

\section{Synthesis of 5}
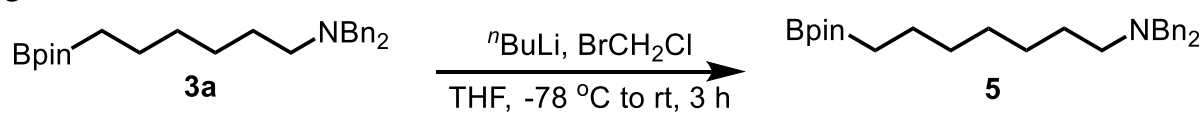

Following a modified procedure ${ }^{33}$, a Schlenk tube equipped with a magnetic stirring bar was evacuated and backfilled with nitrogen. THF $(0.6 \mathrm{~mL}), \mathrm{BrCH}_{2} \mathrm{Cl}(51.6 \mathrm{mg}, 0.40 \mathrm{mmol})$, and $3 \mathrm{a}(81.4 \mathrm{mg}, 0.20 \mathrm{mmol})$ were added to the tube, and the tube was cooled to $-78^{\circ} \mathrm{C}$. A hexane solution of $n$-BuLi $(2.5 \mathrm{M}, 0.12 \mathrm{ml}, 0.30 \mathrm{mmol})$ was added slowly to the mixture via syringe and the resulting solution was stirred at $-78{ }^{\circ} \mathrm{C}$. The mixture was stirred at room temperature for $3 \mathrm{~h}$. $\mathrm{NH}_{4} \mathrm{Cl}(1.0 \mathrm{~mL})$ was added to the tube. The organic materials were extracted with $\mathrm{Et}_{2} \mathrm{O}$, washed with aq. $\mathrm{NaCl}$ and dried over anhydrous $\mathrm{MgSO}_{4}$ and concentrated under vacuo. The residue was purified by silica gel chromatography to afford compound 5 as a colorless oil in $64 \%$ yield $(53.2 \mathrm{mg}$, petroleum ether/EtOAc $=40 / 1)$. $\mathbf{R}_{f}=0.35$ (petroleum ether/EtOAc $=20 / 1$ ) 
${ }^{1} \mathrm{H}$ NMR (400 MHz, $\left.\mathbf{C D C l}_{3}\right) \delta 7.40$ - $7.36(\mathrm{~m}, 4 \mathrm{H}), 7.33-7.28(\mathrm{~m}, 4 \mathrm{H}), 7.25-7.20(\mathrm{~m}, 2 \mathrm{H})$, $3.55(\mathrm{~s}, 4 \mathrm{H}), 2.43-2.38(\mathrm{~m}, 2 \mathrm{H}), 1.54-1.46(\mathrm{~m}, 2 \mathrm{H}), 1.44-1.30(\mathrm{~m}, 4 \mathrm{H}), 1.27-1.22(\mathrm{~m}, 16 \mathrm{H})$, $0.80-0.71(\mathrm{~m}, 2 \mathrm{H})$.

${ }^{13} \mathrm{C}$ NMR (100 MHz, $\left.\mathrm{CDCl}_{3}\right) \delta$ 140.2, 128.9, 128.2, 126.8, 83.0, 58.4, 53.6, 32.6, 29.4, 27.3, 27.2, $27.1,25.0,24.1$.

${ }^{11 B}$ NMR (128 MHz, $\left.\mathrm{CDCl}_{3}\right) \delta 34.1$.

The carbon signal attached to $B$ was not observed due to low intensity.

HRMS (ESI-TOF) calcd for $\mathrm{C}_{27} \mathrm{H}_{41} \mathrm{BNO}_{2}{ }^{+}[\mathrm{M}+\mathrm{H}]^{+}$: 422.3225 , found: 422.3232 .

IR (neat, cm-1): 2926, 1558, 1507, 1457, 1374, 1145, 744, 698.

\section{Synthesis of 6}

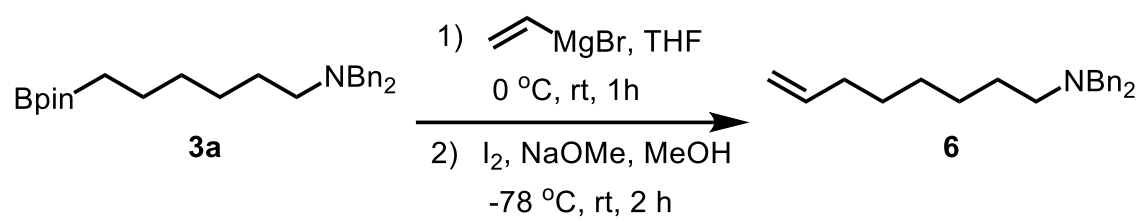

Following the literature procedures ${ }^{34}$, to a $25-\mathrm{mL}$ flame-dried Schlenk tube charged with 3a $(81.4 \mathrm{mg}, 0.20 \mathrm{mmol})$ andTHF $(1.5 \mathrm{~mL})$ was added vinylmagnesium bromide $(1.2 \mathrm{~mL}, 1.0$ $\mathrm{M}$ in THF, 6.0 equiv) dropwisely. The resulted mixture was allowed to stir at room tempereture for one hour. To the above solution at $-78{ }^{\circ} \mathrm{C}$, iodine $(304.8 \mathrm{mg}, 1.2 \mathrm{mmol}, 6.0$ equiv) was added dropwisely as a solution in methanol $(2.0 \mathrm{~mL})$. The reaction mixture was allowed to stir $30 \mathrm{~min}$. at the same temperature followed by dropwise addition of a solution of $\mathrm{NaOMe}(64.8 \mathrm{mg}, 1.2 \mathrm{mmol}, 6.0$ equiv) in methanol $(2.5 \mathrm{~mL})$. After warming to room temperature, the resultant mixture was allowed to stir for $1.5 \mathrm{~h}$. Saturated aq. $\mathrm{Na}_{2} \mathrm{~S}_{2} \mathrm{O}_{3}(2 \mathrm{~mL})$ was then added. The reaction mixture was diluted with $\mathrm{Et}_{2} \mathrm{O}(15 \mathrm{~mL})$ and water $(15 \mathrm{~mL})$. The combined ethereal solution was dried over anhydrous $\mathrm{MgSO}_{4}$. After removal of the solvent, the residue was purified by column chromatography on silica using PE/EtOAc (20/1) as the eluent to afford compound 6 as colorless oil (45.3 $\mathrm{mg}$, 74\% yield)

$\mathbf{R}_{f}=0.45$ (petroleum ether $/$ EtOAc $=20 / 1$ )

${ }^{1}$ H NMR (400 MHz, $\left.\mathbf{C D C l}_{3}\right) \delta 7.44-7.40(\mathrm{~m}, 4 \mathrm{H}), 7.38-7.33(\mathrm{~m}, 4 \mathrm{H}), 7.29-7.25(\mathrm{~m}, 2 \mathrm{H})$, $5.91-5.63(\mathrm{~m}, 1 \mathrm{H}), 5.09-4.93(\mathrm{~m}, 2 \mathrm{H}), 3.60(\mathrm{~s}, 4 \mathrm{H}), 2.50-2.41(\mathrm{~m}, 2 \mathrm{H}), 2.18-1.95(\mathrm{~m}, 2 \mathrm{H})$, $1.59-1.52(\mathrm{~m}, 2 \mathrm{H}), 1.39-1.25(\mathrm{~m}, 6 \mathrm{H})$.

${ }^{13} \mathrm{C}$ NMR (100 MHz, $\left.\mathrm{CDCl}_{3}\right) \delta$ 140.2, 139.3, 128.9, 128.2, 126.8, 114.3, 58.4, 53.5, 33.9, 29.11, 29.06, 27.2, 27.1 .

HRMS (ESI-TOF) calcd for $\mathrm{C}_{22} \mathrm{H}_{30} \mathrm{~N}^{+}[\mathrm{M}+\mathrm{H}]^{+}: 308.2373$, found: 308.2376.

IR (neat, cm-1): 2926, 1684, 1653, 1507, 1374, 1145, 744, 698. 


\section{Synthesis of 7}

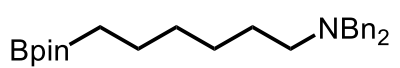

$3 a$

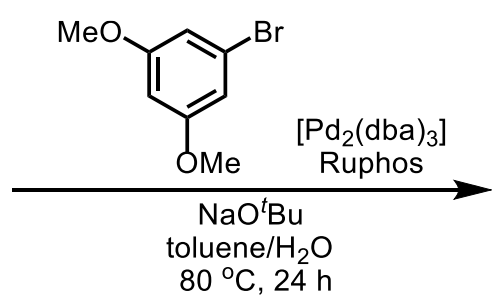

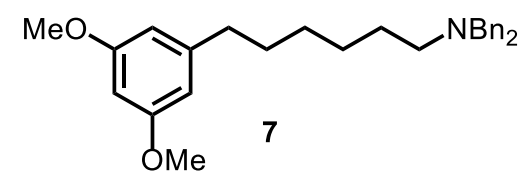

Following a modified procedure ${ }^{35}, \mathrm{Pd}_{2}(\mathrm{dba})_{3}(3.7 \mathrm{mg}, 2 \mathrm{~mol} \%), \mathrm{NaO}{ }^{t} \mathrm{Bu}(57.6 \mathrm{mg}, 0.6$ $\mathrm{mmol})$, Ruphos (3.7 mg, $4 \mathrm{~mol} \%$ ) and compound 3a (97.7 mg, $0.24 \mathrm{mmol})$ were added to a 10 mL Schlenk tube equipped with a stir bar. The vessel was evacuated and filled with $\mathrm{N}_{2}$ (three cycles). THF (1.0 mL), $\mathrm{H}_{2} \mathrm{O}(0.1 \mathrm{~mL})$ and aryl bromide $(43.4 \mathrm{mg}, 0.20 \mathrm{mmol})$ were added in turn by syringe under an $\mathrm{N}_{2}$ atmosphere. The resulting reaction mixture was stirred vigorously at $80^{\circ} \mathrm{C}$ in an oil bath for $24 \mathrm{~h}$. The reaction mixture was then diluted with EtOAc $(5 \mathrm{~mL})$, and the reaction mixture was sequentially washed with water $(5 \mathrm{~mL})$ and saturated aqueous $\mathrm{NaCl}(5 \mathrm{~mL})$, dried over anhydrous $\mathrm{Na}_{2} \mathrm{SO}_{4}$, and concentrated under vacuum. The residue was purified by silica gel chromatography to afford compound 7 as a colorless oil in $62 \%$ yield $(51.1 \mathrm{mg}$, petroleum ether $/ \mathrm{EtOAc}=40 / 1)$.

$\mathbf{R}_{f}=0.50$ (petroleum ether/EtOAc $=10 / 1$ )

${ }^{1}$ H NMR (400 MHz, $\left.\mathbf{C D C l}_{3}\right) \delta 7.39$ - $7.36(\mathrm{~m}, 4 \mathrm{H}), 7.33-7.29(\mathrm{~m}, 4 \mathrm{H}), 7.26-7.21(\mathrm{~m}, 2 \mathrm{H})$, $6.36-6.33(\mathrm{~m}, 2 \mathrm{H}), 6.34-6.30(\mathrm{~m}, 1 \mathrm{H}), 3.79(\mathrm{~s}, 6 \mathrm{H}), 3.56(\mathrm{~s}, 4 \mathrm{H}), 2.54-2.47(\mathrm{~m}, 2 \mathrm{H}), 2.43-$ $2.39(\mathrm{~m}, 2 \mathrm{H}), 1.60-1.50(\mathrm{~m}, 4 \mathrm{H}), 1.33-1.28(\mathrm{~m}, 4 \mathrm{H})$.

${ }^{13} \mathrm{C}$ NMR (100 MHz, $\left.\mathrm{CDCl}_{3}\right) \delta 160.80,145.45,128.99,128.37,128.30,126.98,106.61,97.69,58.37$, $55.37,53.35,36.41,31.41,29.28,27.22,24.96$.

HRMS (ESI-TOF) calcd for $\mathrm{C}_{28} \mathrm{H}_{36} \mathrm{NO}_{2}{ }^{+}[\mathrm{M}+\mathrm{H}]^{+}$: 418.2741, found: 418.2748.

IR (neat, cm-1): 2926, 1653, 1521, 1457, 1205, 1149, 743, 698. 


\section{Control Experiments}
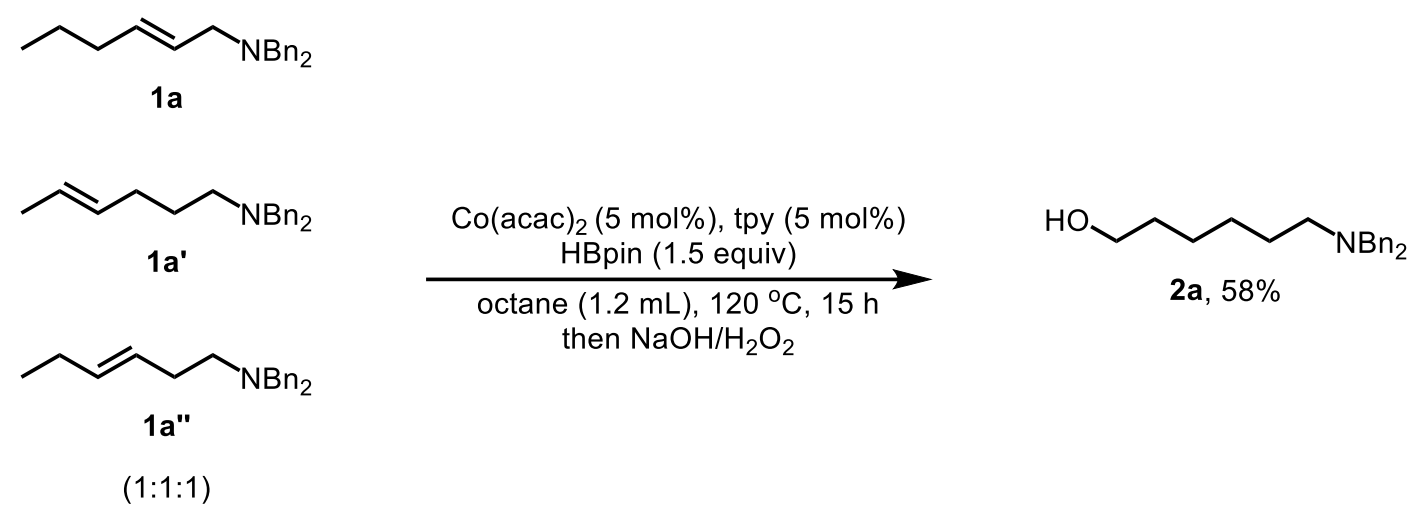

$2 a, 58 \%$

In a glove box, to an oven-dried 10-mL vial were added Co(acac)2 (3.9 mg, $0.015 \mathrm{mmol})$, tpy $(3.5 \mathrm{mg}, 0.015 \mathrm{mmol})$ and anhydrous octane $(1.2 \mathrm{~mL})$. The resulting solution was stirred for $20 \mathrm{~min}$ at room temperature, at which time HBpin $(57.6 \mathrm{mg}, 0.45 \mathrm{mmol})$ was added. After the resulting mixture was stirred for an additional $5 \mathrm{~min}$, the mixture of $(E)-N, N$-dibenzylhex2-en-1-amine 1a (27.9 mg, $0.10 \mathrm{mmol}),(E)-N, N$-dibenzylhex-4-en-1-amine 1a' (27.9 mg, 0.10 mmol) and (E)-N,N-dibenzylhex-3-en-1-amine 1a" (27.9 mg, $0.10 \mathrm{mmol})$ was added and stirred for $15 \mathrm{~h}$ at $120{ }^{\circ} \mathrm{C}$ in an oil bath. The resulting suspension was cooled to room temperature, filtered through a celite pad with EtOAc and concentrated under vacuo. The residue was dissolved in THF $(1.0 \mathrm{~mL})$ and cooled to $0{ }^{\circ} \mathrm{C}$. $\mathrm{NaOH}(1.5$ equiv, $3.0 \mathrm{M})$ and $\mathrm{H}_{2} \mathrm{O}_{2}$ (1.5 equiv, 30\%) were then added. The resulting mixture was stirred for $0.5 \mathrm{~h}$, and extracted with EtOAc, dried with $\mathrm{Na}_{2} \mathrm{SO}_{4}$, filtered and concentrated. The residue was purified by silica gel chromatography to afford compound 3a as a colorless oil in 58\% yield (51.3 mg, petroleum ether $/ \mathrm{EtOAc}=5 / 1)$.

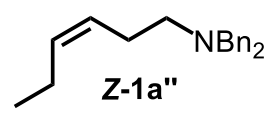

$$
\begin{gathered}
\underset{\mathrm{Co}(\mathrm{acac})_{2}(5 \mathrm{~mol} \%), \text { tpy }(5 \mathrm{~mol} \%)}{\mathrm{HBpin}(1.5 \text { equiv })} \\
\underset{\text { octane }(1.2 \mathrm{~mL}), 120^{\circ} \mathrm{C}, 15 \mathrm{~h}}{\text { then } \mathrm{NaOH} / \mathrm{H}_{2} \mathrm{O}_{2}}
\end{gathered}
$$

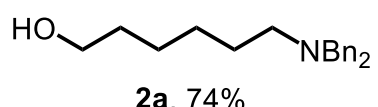

2a, $74 \%$

In a glove box, to an oven-dried 10-mL vial were added Co(acac)2 $(6.5 \mathrm{mg}, 0.025 \mathrm{mmol})$, tpy $(5.9 \mathrm{mg}, 0.025 \mathrm{mmol})$ and anhydrous octane $(2.0 \mathrm{~mL})$. The resulting solution was stirred for $20 \mathrm{~min}$ at room temperature, at which time HBpin $(96.0 \mathrm{mg}, 0.75 \mathrm{mmol})$ was added. After the resulting mixture was stirred for an additional $5 \mathrm{~min}$, the mixture of (Z)-N,Ndibenzylhex-3-en-1-amine 1a" (139.5 mg, $0.50 \mathrm{mmol}$ ) was added and stirred for $15 \mathrm{~h}$ at 120 ${ }^{\circ} \mathrm{C}$ in an oil bath. The resulting suspension was cooled to room temperature, filtered through a celite pad with EtOAc and concentrated under vacuo. The residue was dissolved in THF 
(2.0 mL) and cooled to $0{ }^{\circ} \mathrm{C}$. $\mathrm{NaOH}(1.5$ equiv, $3.0 \mathrm{M})$ and $\mathrm{H}_{2} \mathrm{O}_{2}(1.5$ equiv, $30 \%)$ were then added. The resulting mixture was stirred for $0.5 \mathrm{~h}$, and extracted with EtOAc, dried with $\mathrm{Na}_{2} \mathrm{SO}_{4}$, filtered and concentrated. The residue was purified by silica gel chromatography to afford compound $3 \mathbf{a}$ as a colorless oil in $74 \%$ yield $(111.0 \mathrm{mg}$, petroleum ether/EtOAc $=5 / 1)$.

\section{Deuterium-labeling Experiment}
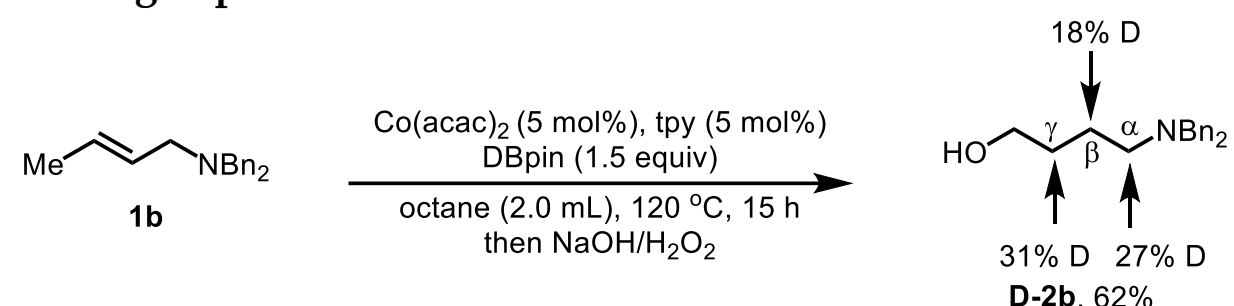

In a glove box, to an oven-dried 10-mL vial were added Co(acac) $2(6.5 \mathrm{mg}, 0.025 \mathrm{mmol})$, tpy $(5.9 \mathrm{mg}, 0.025 \mathrm{mmol})$ and anhydrous octane $(2.0 \mathrm{~mL})$. The resulting solution was stirred for $20 \mathrm{~min}$ at room temperature, at which time DBpin $(96.8 \mathrm{mg}, 0.75 \mathrm{mmol})$ was added. After the resulting mixture was stirred for an additional $5 \mathrm{~min}$, the mixture of $(E)-\mathrm{N}, \mathrm{N}$-dibenzylbut2-en-1-amine $1 \mathbf{b}$ (125.7 mg, $0.50 \mathrm{mmol}$ ) was added and stirred for $15 \mathrm{~h}$ at $120{ }^{\circ} \mathrm{C}$ in an oil bath. The resulting suspension was cooled to room temperature, filtered through a celite pad with EtOAc and concentrated under vacuo. The residue was dissolved in THF $(2.0 \mathrm{~mL})$ and cooled to $0{ }^{\circ} \mathrm{C}$. $\mathrm{NaOH}$ (1.5 equiv, $3.0 \mathrm{M}$ ) and $\mathrm{H}_{2} \mathrm{O}_{2}(1.5$ equiv, 30\%) were then added. The resulting mixture was stirred for $0.5 \mathrm{~h}$, and extracted with EtOAc, dried with $\mathrm{Na}_{2} \mathrm{SO}_{4}$, filtered and concentrated. The residue was purified by silica gel chromatography to afford compound $\mathbf{D}$ $\mathbf{2 b}$ as a colorless oil in $62 \%$ yield $(83.5 \mathrm{mg}$, petroleum ether $/ \mathrm{EtOAc}=5 / 1)$.

${ }^{1} \mathrm{H}$ NMR (400 MHz, Acetone) $\delta 7.43(\mathrm{~d}, J=7.1 \mathrm{~Hz}, 4 \mathrm{H}), 7.35(\mathrm{t}, J=7.5 \mathrm{~Hz}, 4 \mathrm{H}), 7.26(\mathrm{t}, J=7.3$ $\mathrm{Hz}, 2 \mathrm{H}), 3.59(\mathrm{~s}, 4 \mathrm{H}), 3.51(\mathrm{t}, J=6.2 \mathrm{~Hz}, 2 \mathrm{H}), 2.51-2.40(\mathrm{~m}, 1.51 \mathrm{H}), 1.68-1.60(\mathrm{~m}, 1.52 \mathrm{H})$, $1.59-1.47(\mathrm{~m}, 1.71 \mathrm{H})$. 


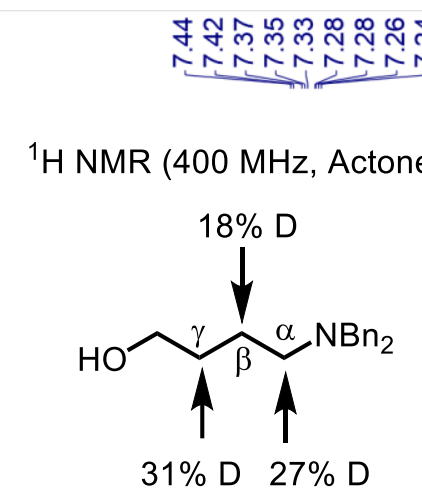

NMR (400 MHz, Actone)

D-2b

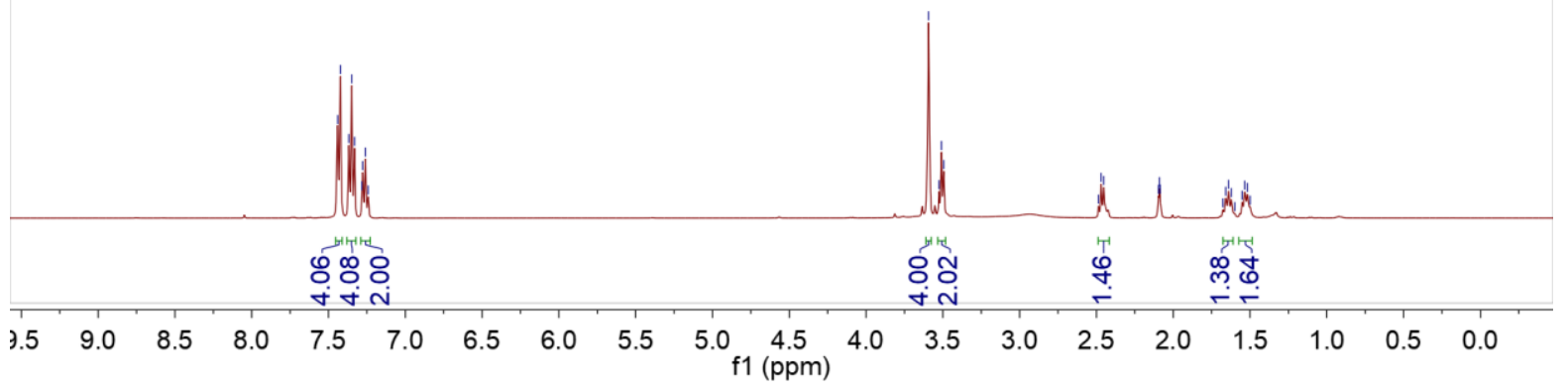

Remote hydroboration of enamine 1ah

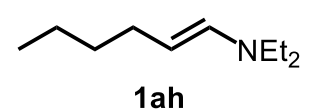

$$
\begin{gathered}
\text { Co(acac })_{2}(5 \mathrm{~mol} \%) \text {, tpy }(5 \mathrm{~mol} \%) \\
\mathrm{HBpin}(1.5 \text { equiv })
\end{gathered}
$$

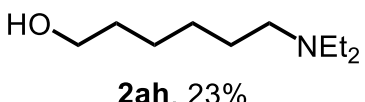

2ah, $23 \%$

In a glove box, to an oven-dried 10-mL vial were added Co(acac)2 $(6.5 \mathrm{mg}, 0.025 \mathrm{mmol})$, tpy $(5.9 \mathrm{mg}, 0.025 \mathrm{mmol})$ and anhydrous octane $(2.0 \mathrm{~mL})$. The resulting solution was stirred for $20 \mathrm{~min}$ at room temperature, at which time HBpin $(96.0 \mathrm{mg}, 0.75 \mathrm{mmol})$ was added. After the resulting mixture was stirred for an additional $5 \mathrm{~min}$, the mixture of $(E)-N, N$-diethylhex1-en-1-amine $1 \mathrm{ah}(77.6 \mathrm{mg}, 0.50 \mathrm{mmol})$ was added and stirred for $15 \mathrm{~h}$ at $120{ }^{\circ} \mathrm{C}$ in an oil bath. The resulting suspension was cooled to room temperature, filtered through a celite pad with EtOAc and concentrated under vacuo. The residue was dissolved in THF $(2.0 \mathrm{~mL})$ and cooled to $0{ }^{\circ} \mathrm{C}$. $\mathrm{NaOH}(1.5$ equiv, $3.0 \mathrm{M})$ and $\mathrm{H}_{2} \mathrm{O}_{2}(1.5$ equiv, 30\%) were then added. The resulting mixture was stirred for $0.5 \mathrm{~h}$, and extracted with EtOAc, dried with $\mathrm{Na}_{2} \mathrm{SO}_{4}$, filtered and concentrated. The residue was purified by base-acid extraction to afford compound $2 \mathrm{ah}$ as a colorless oil in 23\% yield (19.9 mg).

${ }^{1} \mathrm{H}$ NMR (400 MHz, $\left.\mathrm{CDCl}_{3}\right) \delta 3.64(\mathrm{t}, J=6.5 \mathrm{~Hz}, 2 \mathrm{H}), 2.56(\mathrm{q}, J=7.2 \mathrm{~Hz}, 4 \mathrm{H}), 2.47$ - $2.42(\mathrm{~m}$, 2H), $1.61-1.54(\mathrm{~m}, 2 \mathrm{H}), 1.51-1.45(\mathrm{~m}, 2 \mathrm{H}), 1.39-1.30(\mathrm{~m}, 4 \mathrm{H}), 1.04(\mathrm{t}, J=7.2 \mathrm{~Hz}, 6 \mathrm{H})$.

${ }^{13} \mathrm{C}$ NMR (100 MHz, $\left.\mathrm{CDCl}_{3}\right) \delta$ 63.0, 52.7, 46.8, 32.8, 27.4, 25.7, 11.5, 1.2. 
The ${ }^{1} \mathrm{H}$ and ${ }^{13} \mathrm{C}$ NMR spectroscopic data match the reported characterization data. ${ }^{8}$

\section{References}

1. Tessore, F.; Roberto, D.; Ugo R.; Pizzotti M. Inorg. Chem. 2005, 44, 8967-8978.

2. Hierso, J.-C.; Fihri, A.; Amardeil, R.; Meunier, P.; Doucet, H.; Santelli, M. Tetrahedron 2005, 61, 9759-9766.

3. Bao, H.; Qi, X.; Tambar, U. K. J. Am. Chem. Soc. 2011, 133, 1206-1208.

4. Ide, T.; Barham, J. P.; Fujita, M.; Kawato, Y.; Egami, H.; Hamashima, Y. Chem. Sci. 2018, 9, 8453-8460.

5. Ondachi, P. W.; Kormos, C. M.; Runyon, S. P.; Thomas, J. B.; Mascarella, S. W.; Decker, A. M.; Navarro, H. A.; Fennell, T. R.; Snyder, R. W.; Carroll, F. I. J. Med. Chem. 2018, 61, 75257545.

6. Murahashi, S. I.; Imada, Y.; Nishimura, K. Tetrahedron 1994, 50, 453-464.

7. Beholz, L. G.; Stille, J. R. J. Org. Chem. 1993, 58, 5095-5100.

8. Li, J.; Qu, S.; Zhao, W. Angew. Chem. Int. Ed. 2020, 59, 2360-2364.

9. Yu, X.; Wannenmacher, N.; Peters, R. Angew. Chem. Int. Ed. 2020, 59, 10944-10948.

10. Qi, J.; Zhang, F. L.; Huang, Y. S.; Xu, A. Q.; Ren, S. C.; Yi, Z. Y.; Wang, Y. F. Org. Lett. 2018, 20, 2360-2364.

11. Sun, M. R.; Lu, H. T.; Wang, Y. Z.; Yang, H.; Liu, H. M. J. Org. Chem. 2009, 74, 2213-2216.

12. Aggarwal, V. K.; Fang, G. Y.; Charmant, J. P. H.; Meek, G. Org. Lett. 2003, 5, 1757-1760.

13. Tian, J.; Luo, F.; Zhang, Q.; Liang, Y.; Li, D.; Zhan, Y.; Kong, L.; Wang, Z. X.; Peng, B. J. Am. Chem. Soc. 2020, 142, 6884-6890.

14. Ravinder B.; Rajeswar Reddy S.; Panasa Reddy A.; Bandichhor, R. Tetrahedron. Lett. 2013, 54, 4908-4913.

15. Cazorla, C.; Billamboz, M.; Bricout, H.; Monflier, E.; Len, C. Eur. J. Org. Chem. 2017, 2017, 1078-1085.

16. Clay, J. M.; Vedejs, E. J. Am. Chem. Soc. 2005, 127, 5766-5767.

17. Nazari, S. H.; Tiempos-Flores, N.; Forson, K. G.; Bourdeau, J. E.; Michaelis, D. J. J. Org. Chem. 2018, 83, 10646-10654.

18. Nishikata, T.; Lipshutz, B. H. Org. Lett. 2009, 11, 2377-2379.

19. Takamatsu, M.; Terao, Y.; Sekiya, M. Chem. Pharm. Bull. 1982, 30, 2682-2687.

20. Wipf, P.; Maciejewski, J. P. Org. Lett. 2008, 10, 4383-4386.

21. Schwarz, J. L.; Schafers, F.; Tlahuext-Aca, A.; Luckemeier, L.; Glorius, F. J. Am. Chem. Soc. 
2018, 140, 12705-12709.

22. De Vita, D.; Pandolfi, F.; Ornano, L.; Feroci, M.; Chiarotto, I.; Sileno, I.; Pepi, F.; Costi, R.; Di Santo, R.; Scipione, L. J. Enzyme. Inhib. Med. Chem. 2016, 31, 106-113.

23. Luo, L.; Song, Q.; Li, Y.; Cao, Z.; Qiang, X.; Tan, Z.; Deng, Y. Bioorg. Med. Chem. 2020, 28, 115400.

24. Goujon, A.; Colom, A.; Strakova, K.; Mercier, V.; Mahecic, D.; Manley, S.; Sakai, N.; Roux, A.; Matile, S. J. Am. Chem. Soc. 2019, 141, 3380-3384.

25. Cunillera, A.; de los Bernardos, M. D.; Urrutigoïty, M.; Claver, C.; Ruiz, A.; Godard, C. Catal. Sci. Technol. 2020, 10, 630-634.

26. Mamidala, R.; Samser, S.; Sharma, N.; Lourderaj, U.; Venkatasubbaiah, K. Organometallics. 2017, 36, 3343-3351.

27. Ye, G.; Wang, D.; He, Y.; Wang, X. J. Mater. Chem. 2010, 20, 10680.

28. Okamoto, H.; Taniguchi, A.; Usami, S.; Taguchi, A.; Takayama, K.; Hayashi, Y. Chem. Commun. 2019, 55, 9108-9111.

29. Jiao, Z.; Chee, K. W.; Zhou, J. S. J. Am. Chem. Soc. 2016, 138, 16240-16243.

30. Pei, H.; Liu, Y.; Wang, D.; Wang, J.; Wang, C.; Wu, Y.; Pan, W.; Su, C.; Song, M.; Cao, S. ePolymers. 2020, 20, 353-360.

31. Yoritate, M.; Meguro, T.; Matsuo, N.; Shirokane, K.; Sato, T.; Chida, N. Chem. Eur. J. 2014, 20, 8210-8216.

32. Wang, Y. D.; Bai, J. Y.; Yang, Y. Q.; Zhao, W. X.; Liang, Y.; Wang, D.; Zhao, Y.; Shi, Z. Z. Chem. Sci. 2021, 12, 3599-3607.

33. Ohmura, T.; Torigoe, T.; Suginome, M. Chem. Commun. 2014, 50, 6333-6336.

34. Zhao, H.; Gao, Q.; Zhang, Y.; Zhang, P.; Xu, S. Org. Lett. 2020, 22, 2861-2866.

35. Wang, X.; Zhang, S.; Cui, P.; Li, S. Org. Lett. 2020, 22, 8702-8707. 


\section{NMR Spectra}

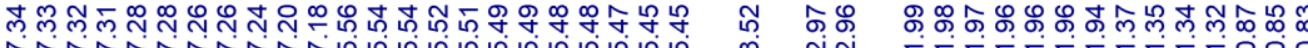

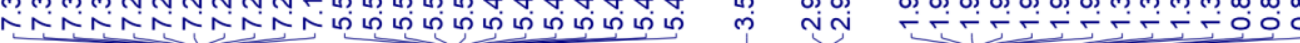

${ }^{1} \mathrm{H}$ NMR $\left(400 \mathrm{MHz}, \mathrm{CDCl}_{3}\right)$

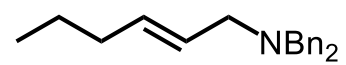

$1 \mathrm{a}$

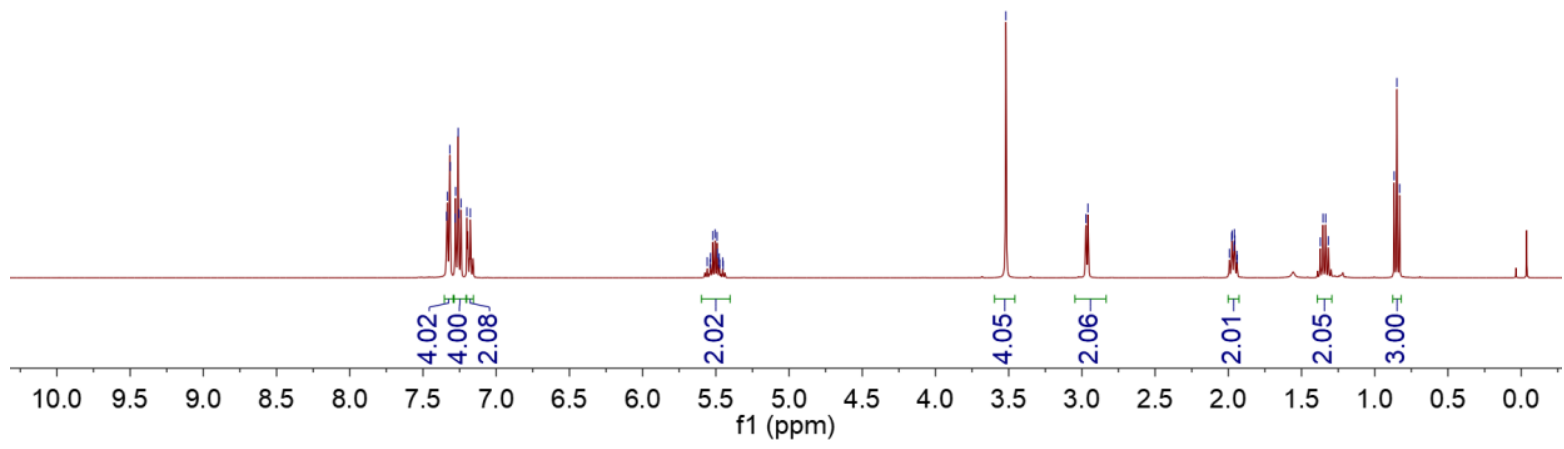

\section{mำ}

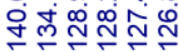

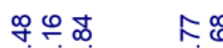

NNe की

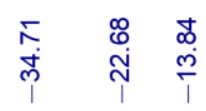

${ }^{13} \mathrm{C}$ NMR $\left(100 \mathrm{MHz}, \mathrm{CDCl}_{3}\right)$

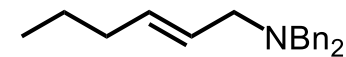

$1 \mathrm{a}$

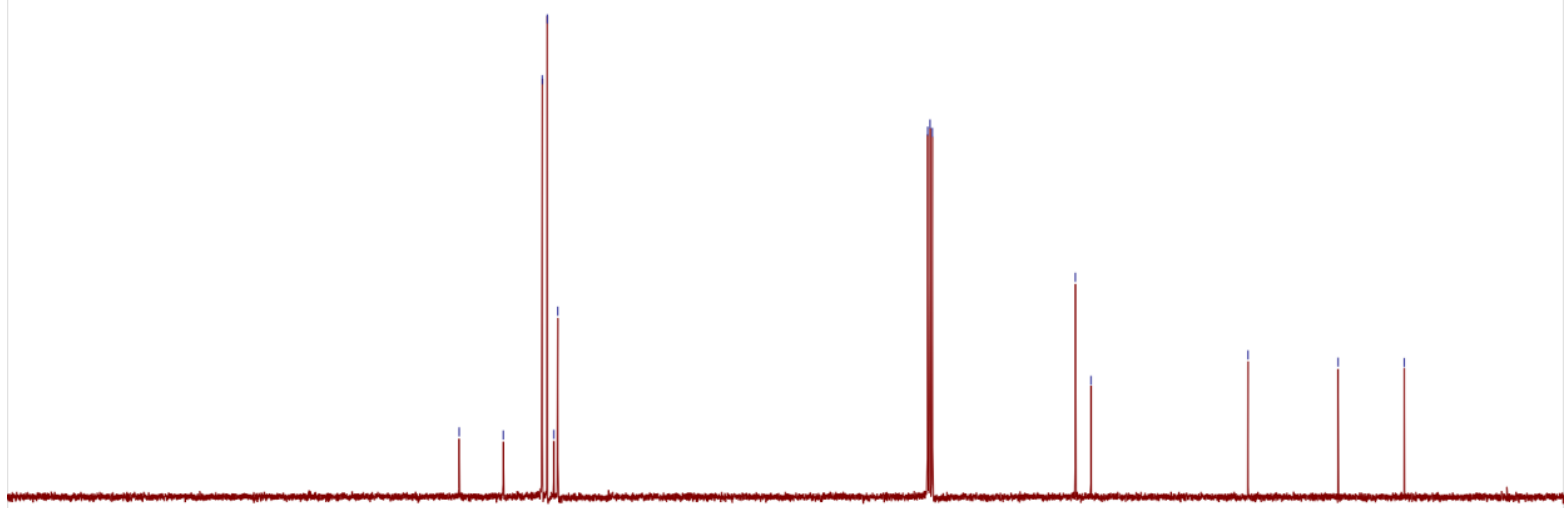

$\begin{array}{llllllllllll}\text { J0 } & 190 & 180 & 170 & 160 & 150 & 140 & 130 & 120 & 110 & 100 & 90\end{array}$

$80 \quad 70 \quad 60$ 
${ }^{1} \mathrm{H}$ NMR $\left(400 \mathrm{MHz}, \mathrm{CDCl}_{3}\right)$

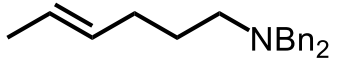

$1 a^{\prime}$

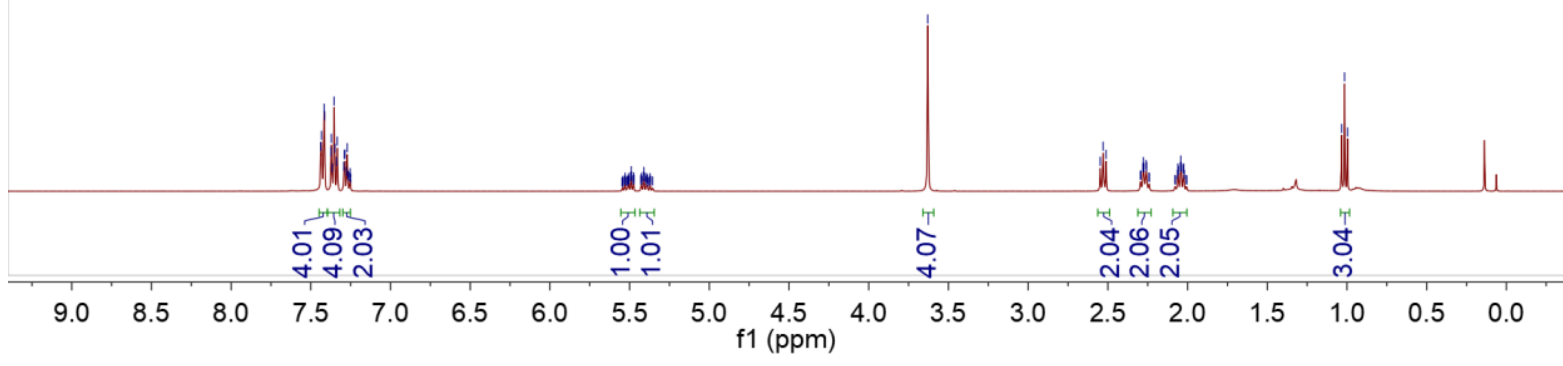

d:

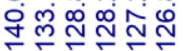

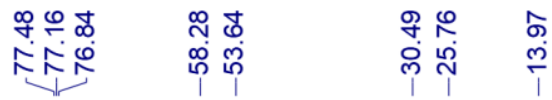

${ }^{13} \mathrm{C}$ NMR $\left(100 \mathrm{MHz}, \mathrm{CDCl}_{3}\right)$
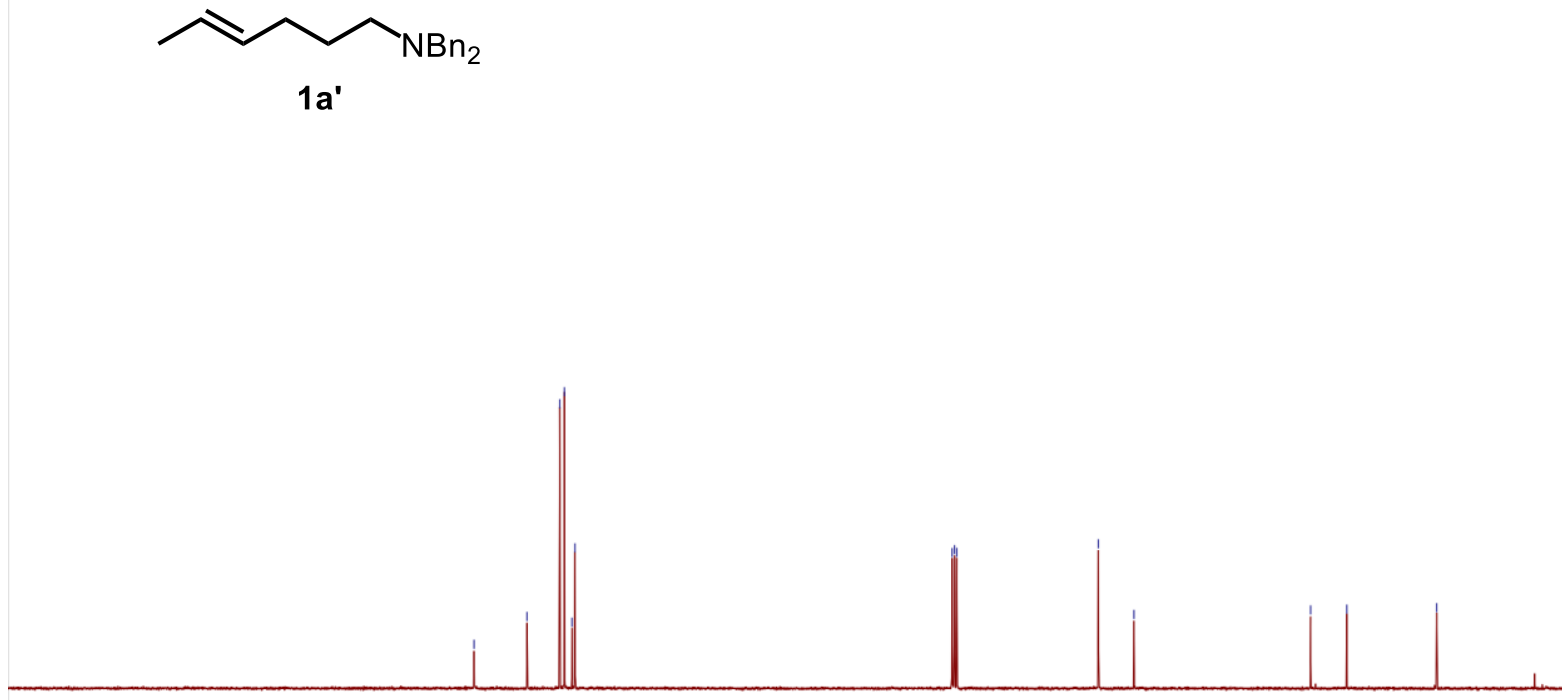

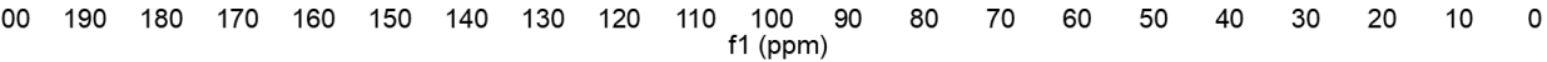


${ }^{1} \mathrm{H}$ NMR $\left(400 \mathrm{MHz}, \mathrm{CDCl}_{3}\right)$

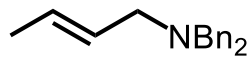

1b

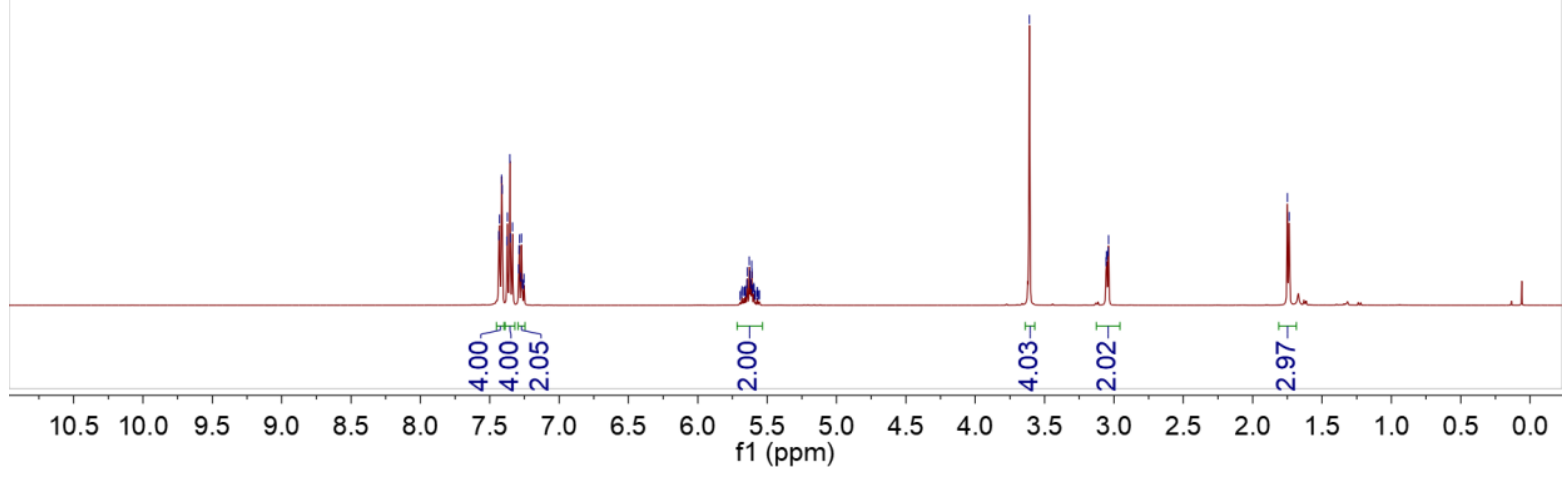

ㅇำำ ๓

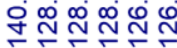

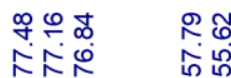

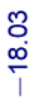

${ }^{13} \mathrm{C}$ NMR $\left(100 \mathrm{MHz}, \mathrm{CDCl}_{3}\right)$<smiles>C/C=C/CNCCCCCCCCCC</smiles>

1b

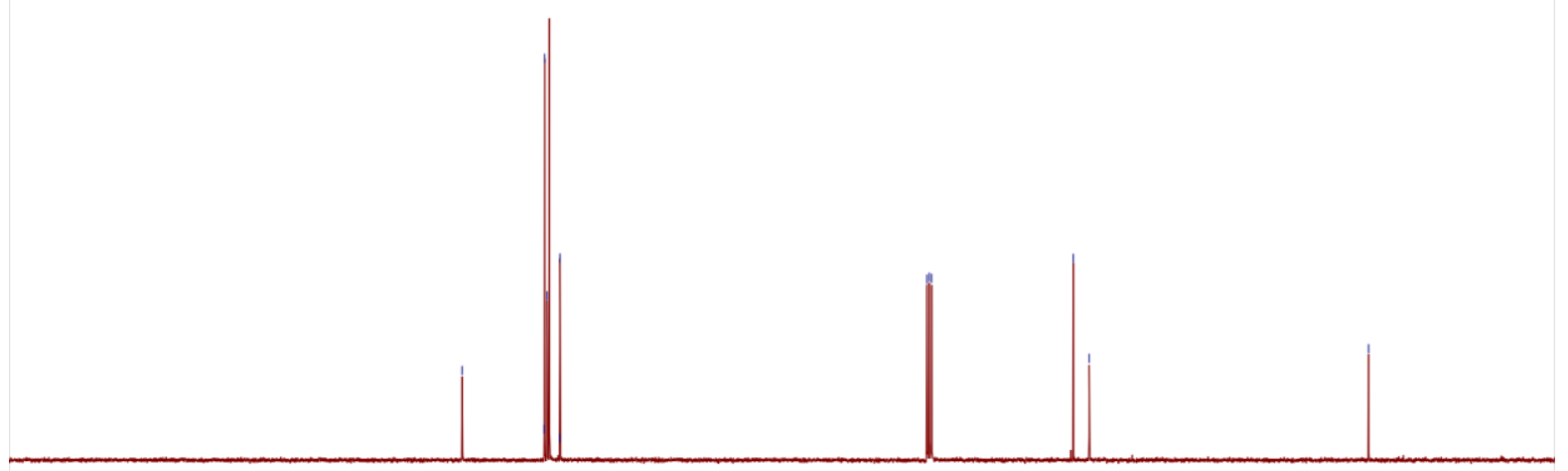

$\begin{array}{lllllllllllllllllllll}00 & 190 & 180 & 170 & 160 & 150 & 140 & 130 & 120 & 110 & 100 & 90 & 80 & 70 & 60 & 50 & 40 & 30 & 20 & 10 & 0\end{array}$ f1 (ppm) 


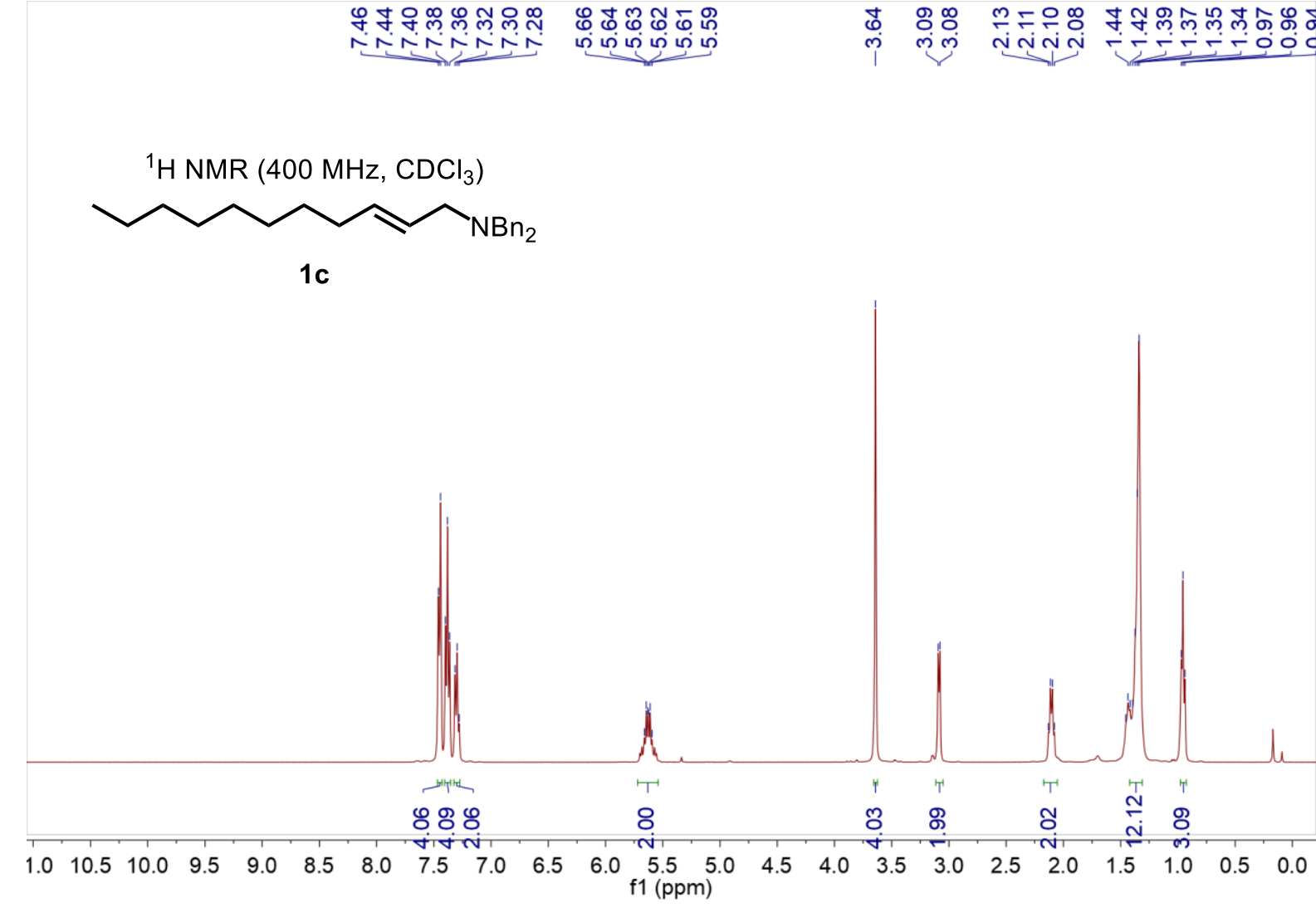

ธำㅇำ

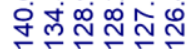

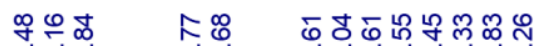

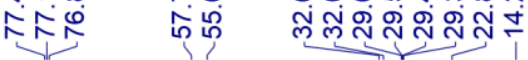

${ }^{13} \mathrm{C}$ NMR (100 MHz, $\left.\mathrm{CDCl}_{3}\right)$

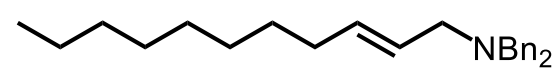

1c

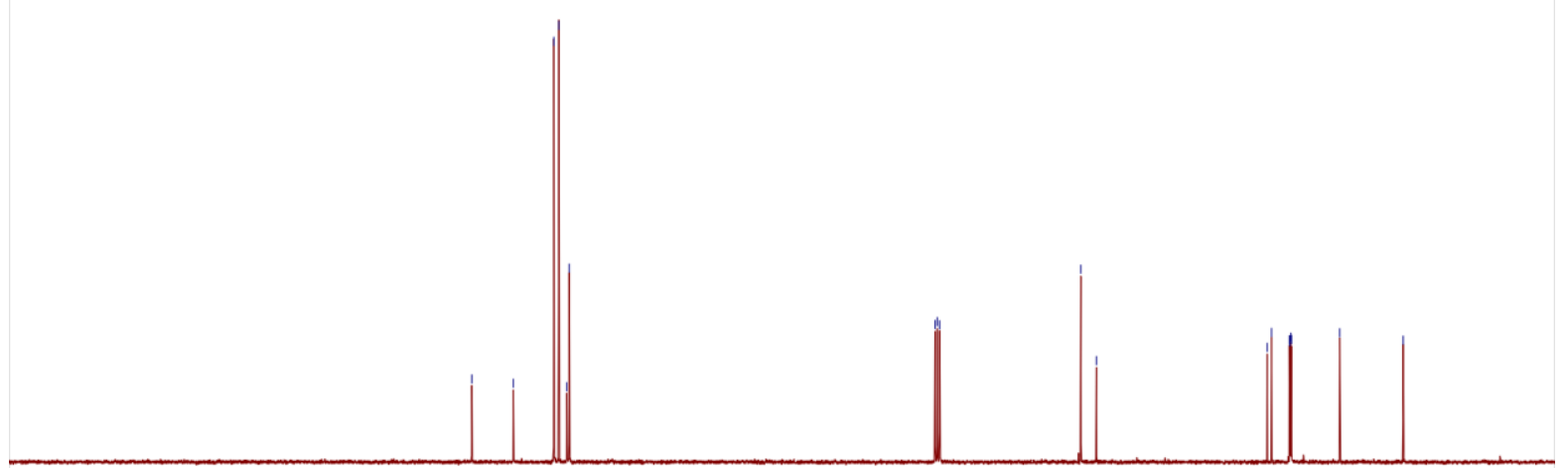


${ }^{1} \mathrm{H} \mathrm{NMR}\left(400 \mathrm{MHz}, \mathrm{CDCl}_{3}\right)$

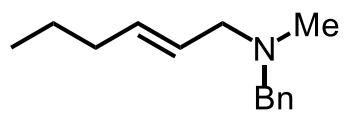

1d

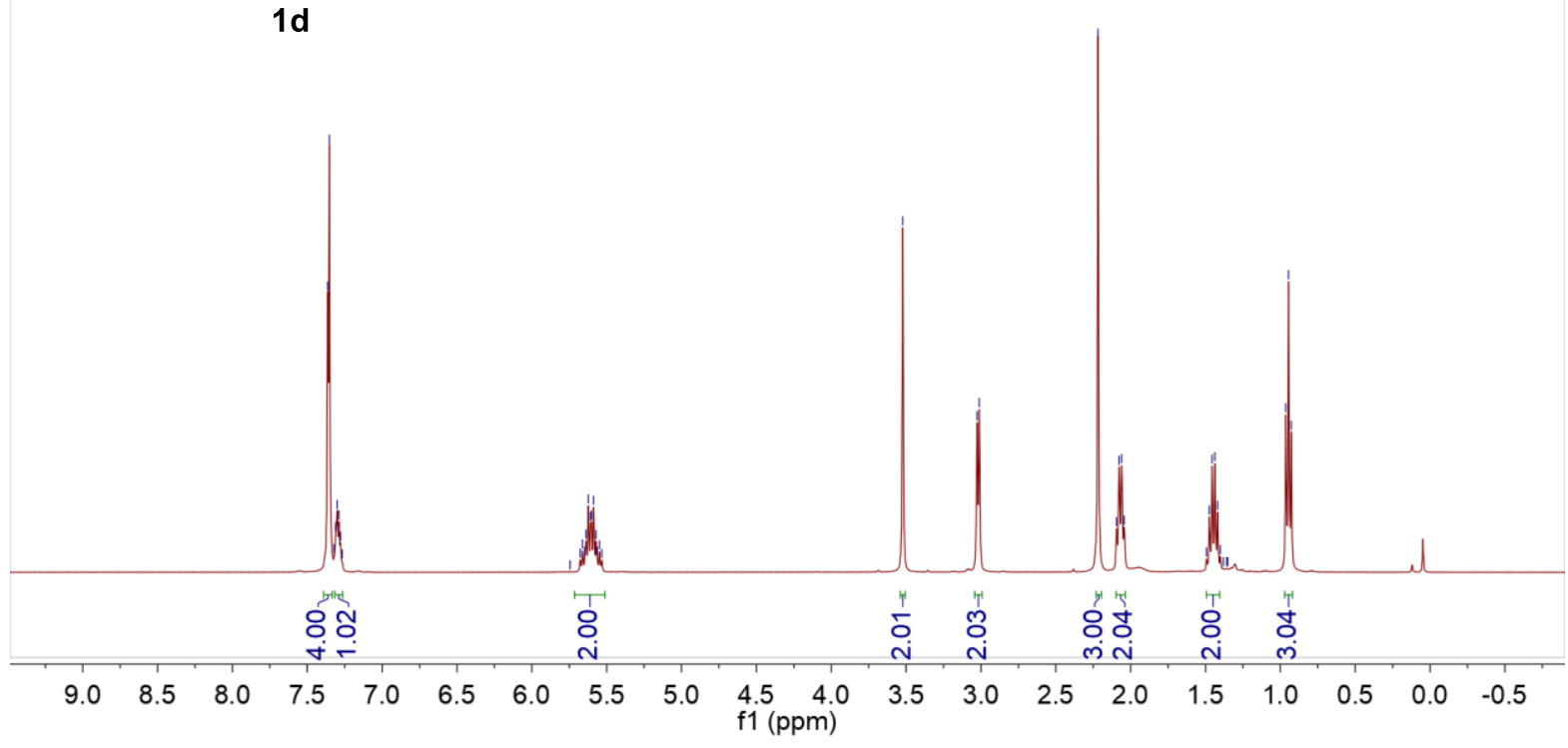

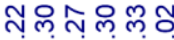

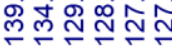

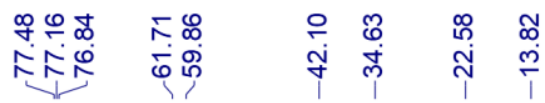

${ }^{13} \mathrm{C} \mathrm{NMR}\left(100 \mathrm{MHz}, \mathrm{CDCl}_{3}\right)$

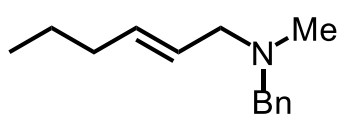

1d

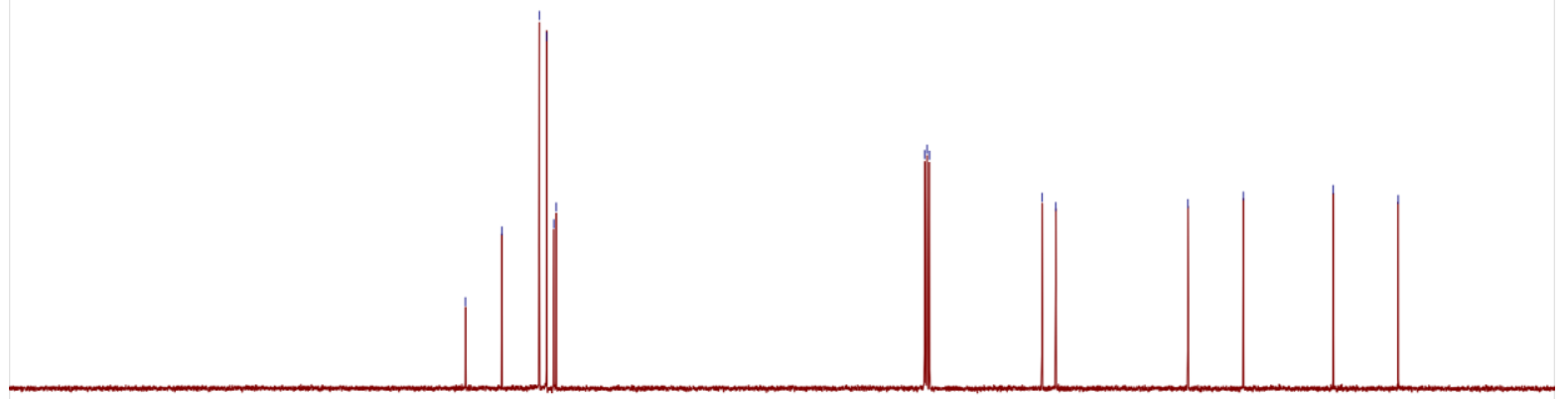

\begin{tabular}{llllllllllllllllllllll}
\hline & 190 & 180 & 170 & 160 & 150 & 140 & 130 & 120 & 110 & 100 & 90 & 80 & 70 & 60 & 50 & 40 & 30 & 20 & 10 & 0 & 0
\end{tabular} f1 (ppm) 
${ }^{1} \mathrm{H}$ NMR $\left(400 \mathrm{MHz}, \mathrm{CDCl}_{3}\right)$

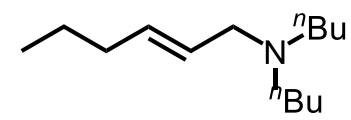

1e

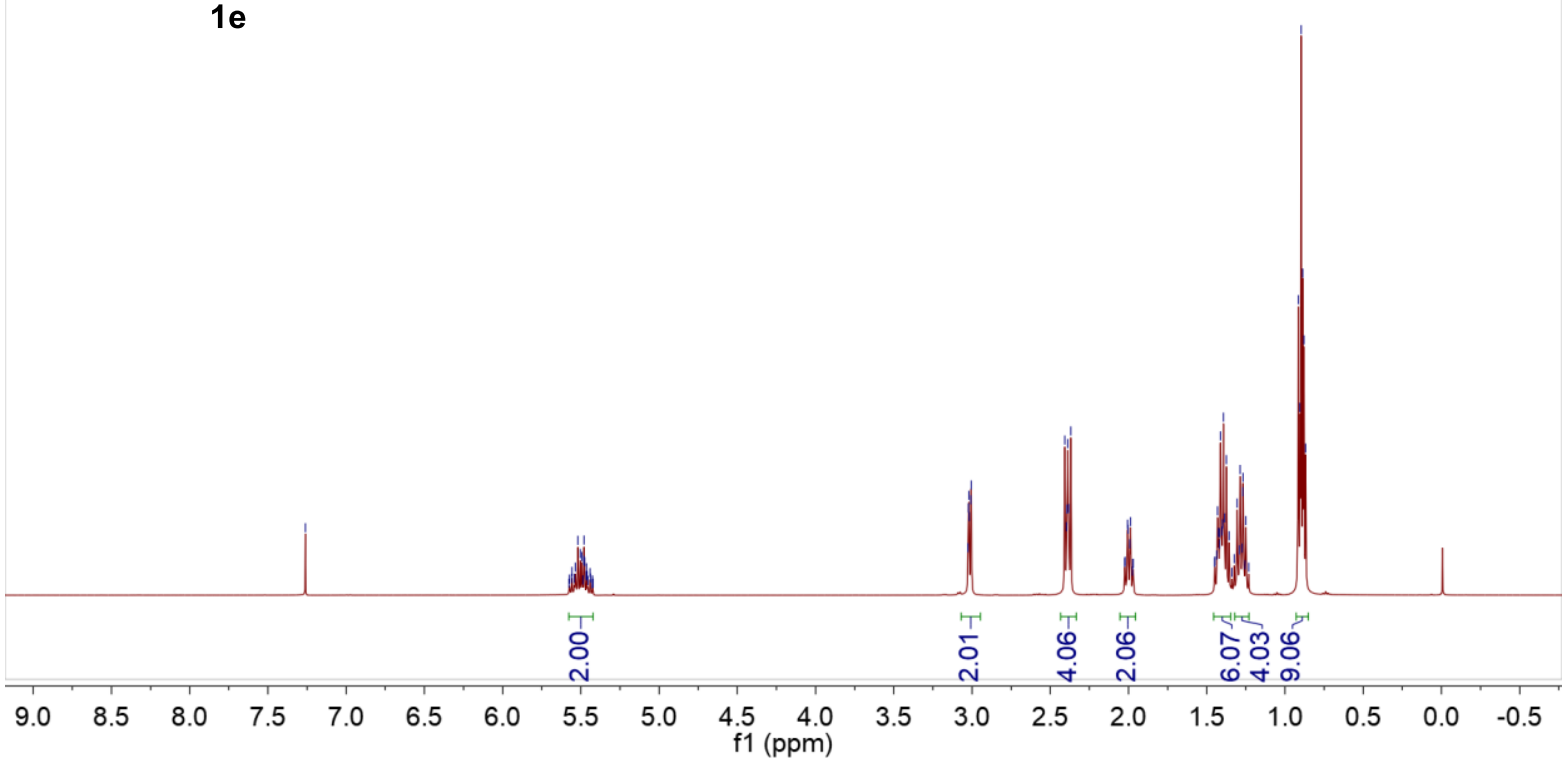

\begin{tabular}{|c|c|c|}
\hline 怘 & 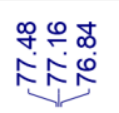 & 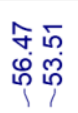 \\
\hline
\end{tabular}

${ }^{13} \mathrm{C}$ NMR $\left(100 \mathrm{MHz}, \mathrm{CDCl}_{3}\right)$<smiles>CCC/C=C/CN(C(=O)CCC)C(=O)c1ccccc1</smiles>

$1 e$

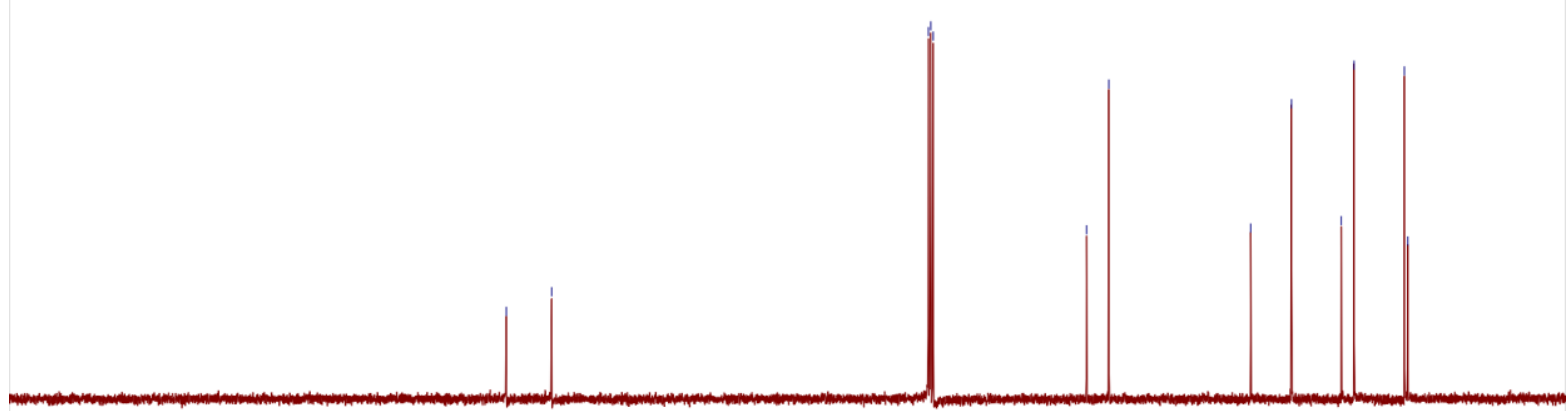

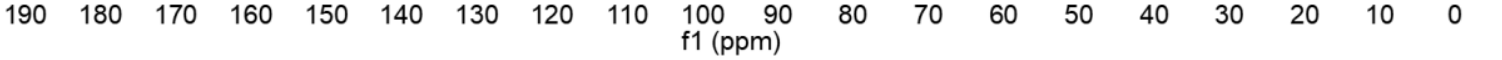




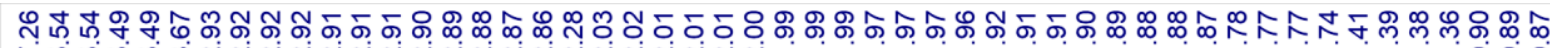

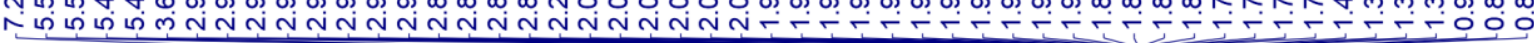

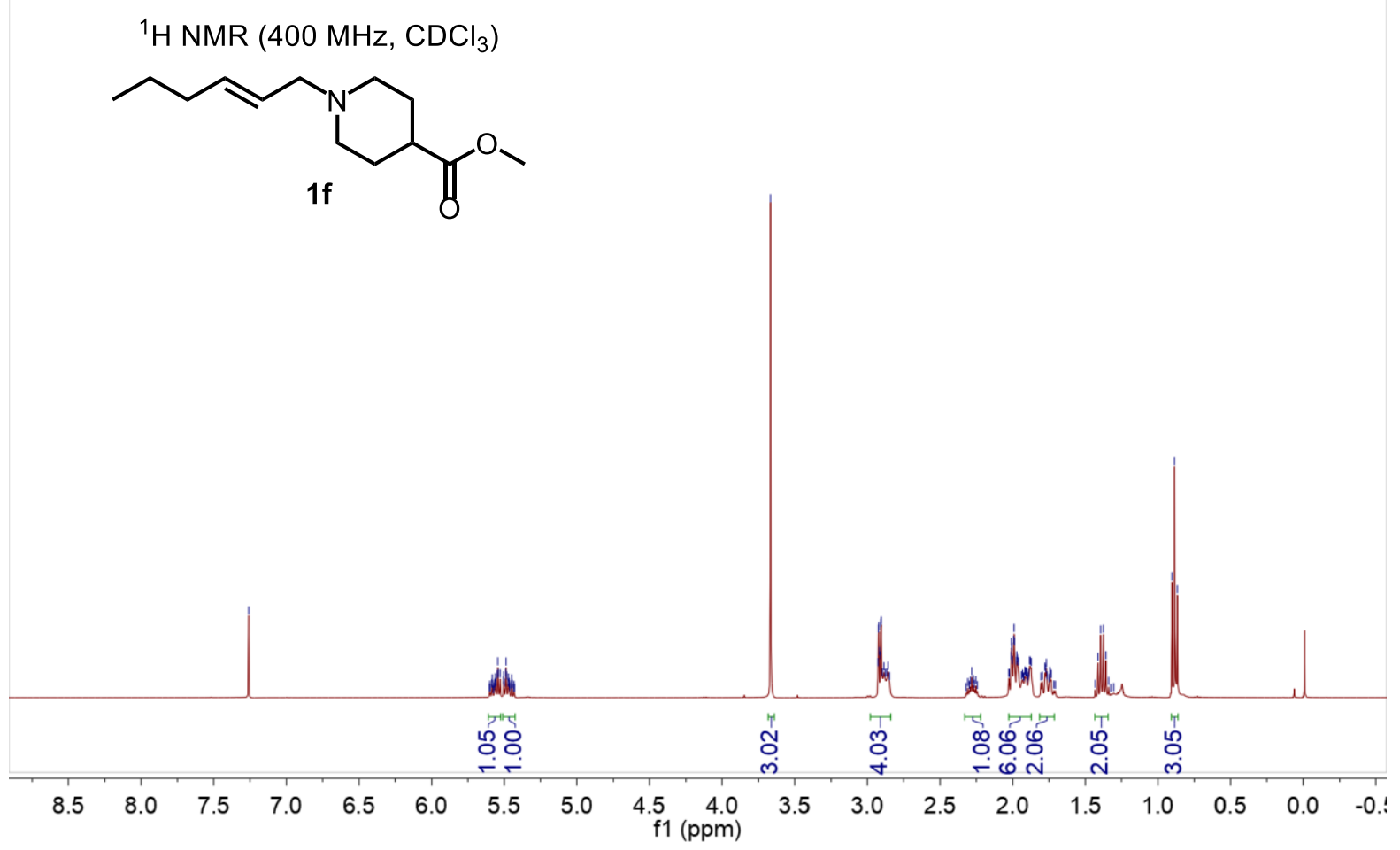

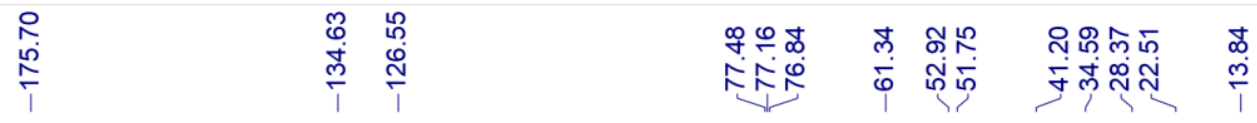

${ }^{13} \mathrm{C} \mathrm{NMR}\left(100 \mathrm{MHz}, \mathrm{CDCl}_{3}\right)$<smiles>CCC/C=C/CN1CCC(C(=O)OC)CC1</smiles>

$\begin{array}{lllllllllllllllllllll}200 & 190 & 180 & 170 & 160 & 150 & 140 & 130 & 120 & 110 & 100 & 90 & 80 & 70 & 60 & 50 & 40 & 30 & 20 & 10 & 0\end{array}$ 


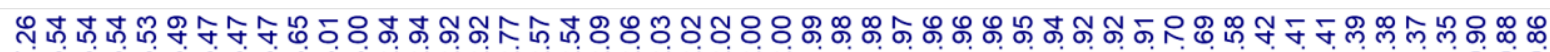

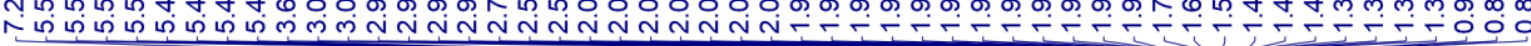

${ }^{1} \mathrm{H}$ NMR $\left(400 \mathrm{MHz}, \mathrm{CDCl}_{3}\right)$

$\overbrace{}^{N^{\prime}}$

$1 \mathrm{~g}$

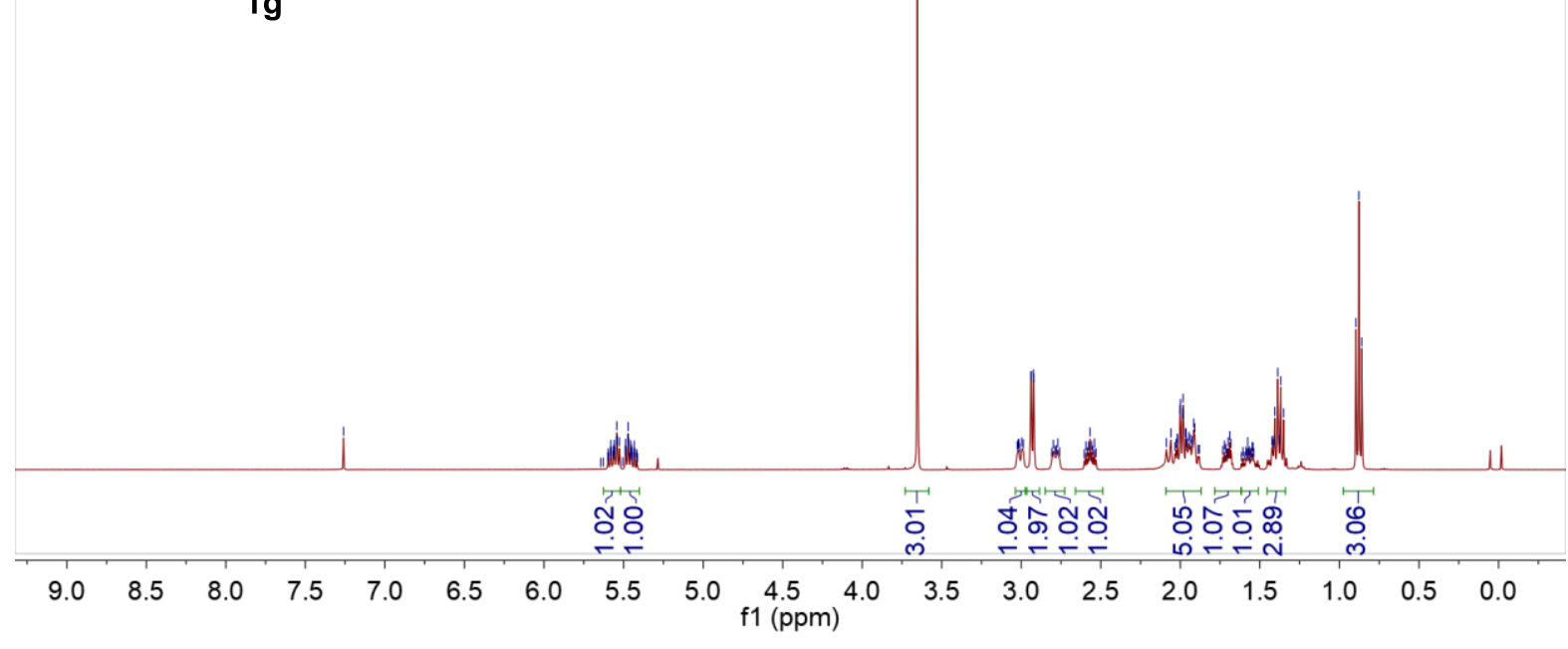

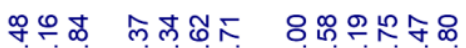

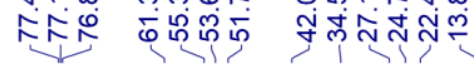

${ }^{13} \mathrm{C}$ NMR $\left(100 \mathrm{MHz}, \mathrm{CDCl}_{3}\right)$<smiles>CCC/C=C/CN1CCC[C@H](C(=O)OC)C1</smiles>

$1 \mathrm{~g}$

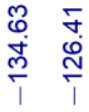


${ }^{1} \mathrm{H}$ NMR $\left(400 \mathrm{MHz}, \mathrm{CDCl}_{3}\right)$<smiles>CCC/C=C/CN1CCC[C@H]1C(=O)OC</smiles>

$1 \mathrm{~h}$

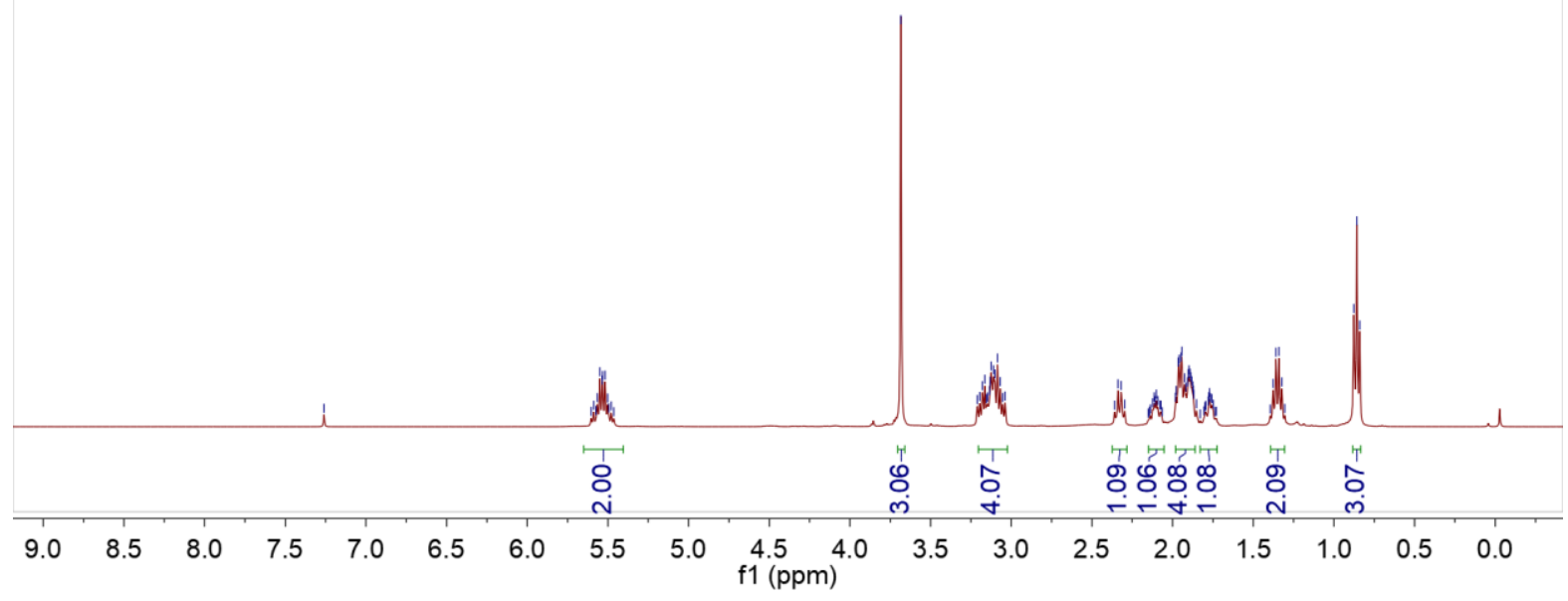

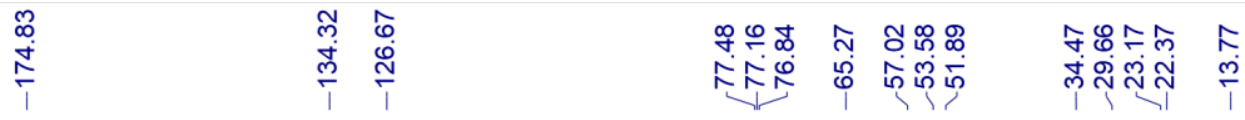

${ }^{13} \mathrm{C} \mathrm{NMR}\left(100 \mathrm{MHz}, \mathrm{CDCl}_{3}\right)$<smiles>CCC/C=C/CN1CCC[C@H]1C(=O)OC</smiles>

$1 \mathrm{~h}$

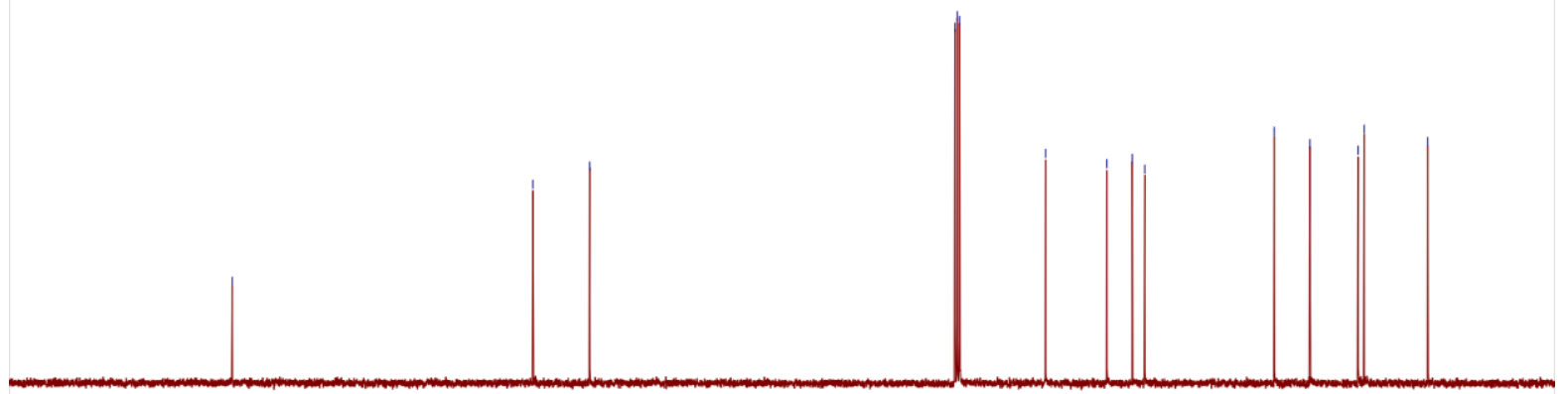

$\begin{array}{lllllllllllllllllllll}200 & 190 & 180 & 170 & 160 & 150 & 140 & 130 & 120 & 110 & 100 & 90 & 80 & 70 & 60 & 50 & 40 & 30 & 20 & 10 & 0\end{array}$ 
${ }^{1} \mathrm{H}$ NMR $\left(400 \mathrm{MHz}, \mathrm{CDCl}_{3}\right)$<smiles>CCC/C=C/CN1CCC2(CC1)OCCO2</smiles>

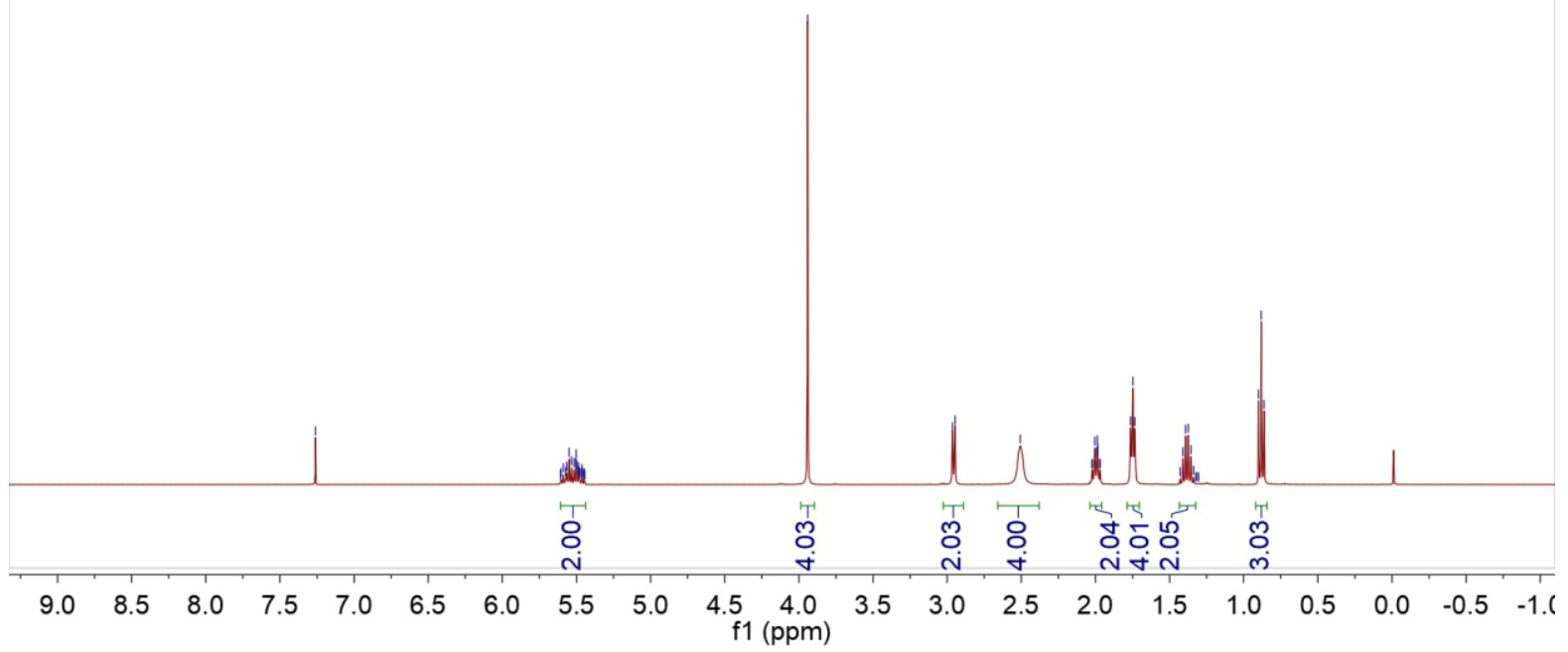

\begin{tabular}{|c|c|c|c|c|c|c|}
\hline 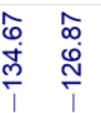 & $\begin{array}{l}\stackrel{0}{\text { م⿱⺈ }} \\
\stackrel{0}{\Gamma}\end{array}$ & 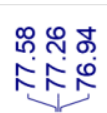 & $\begin{array}{l}\forall \& \\
000 \\
10\end{array}$ & 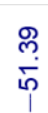 & $\begin{array}{l}9 R \\
\text { pिं } \\
\text { pr }\end{array}$ & $\underset{\mathbb{N}}{\bar{ஸ}}$ \\
\hline
\end{tabular}

${ }^{13} \mathrm{C}$ NMR $\left(100 \mathrm{MHz}, \mathrm{CDCl}_{3}\right)$<smiles>CCC/C=C/CN1CCC2(CC1)OCCO2</smiles>

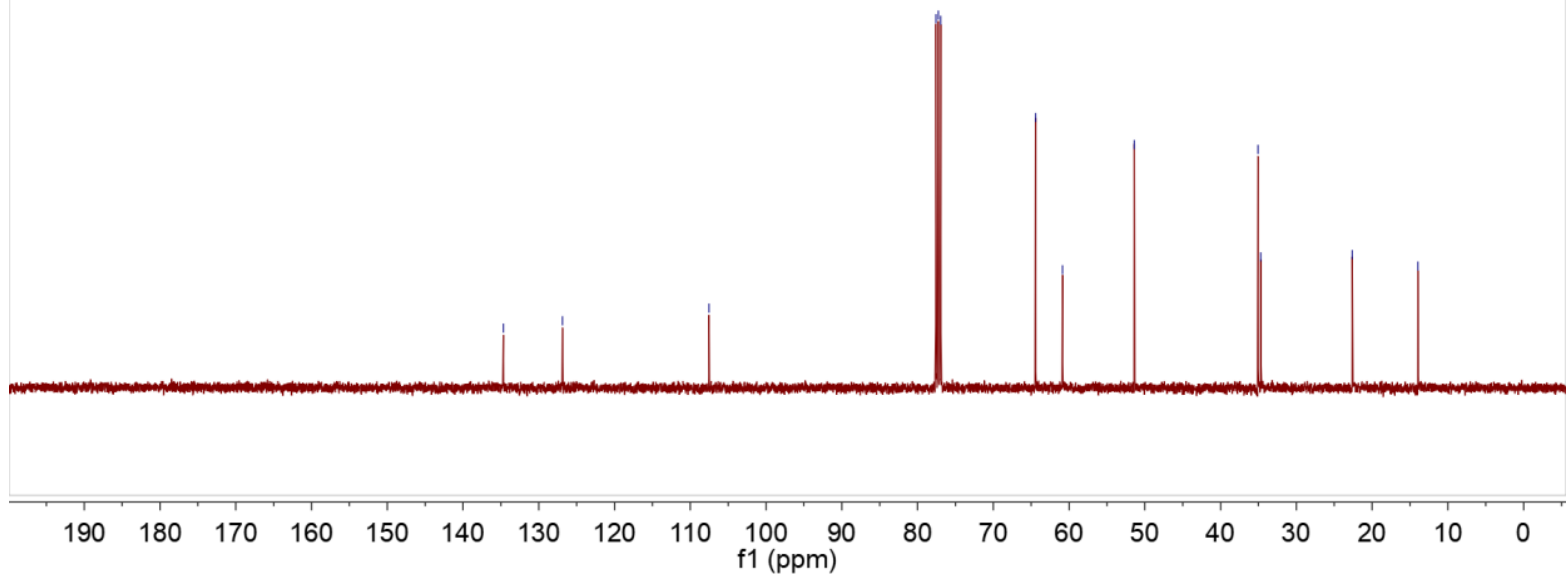




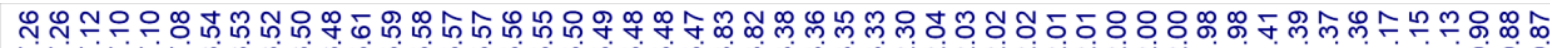

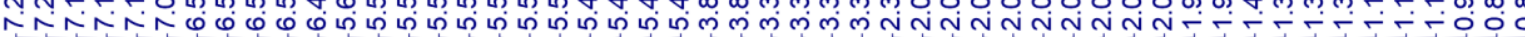
$\longrightarrow$

${ }^{1} \mathrm{H}$ NMR $\left(400 \mathrm{MHz}, \mathrm{CDCl}_{3}\right)$<smiles>CCC/C=C/CN(CC)c1cccc(C)c1</smiles>

10

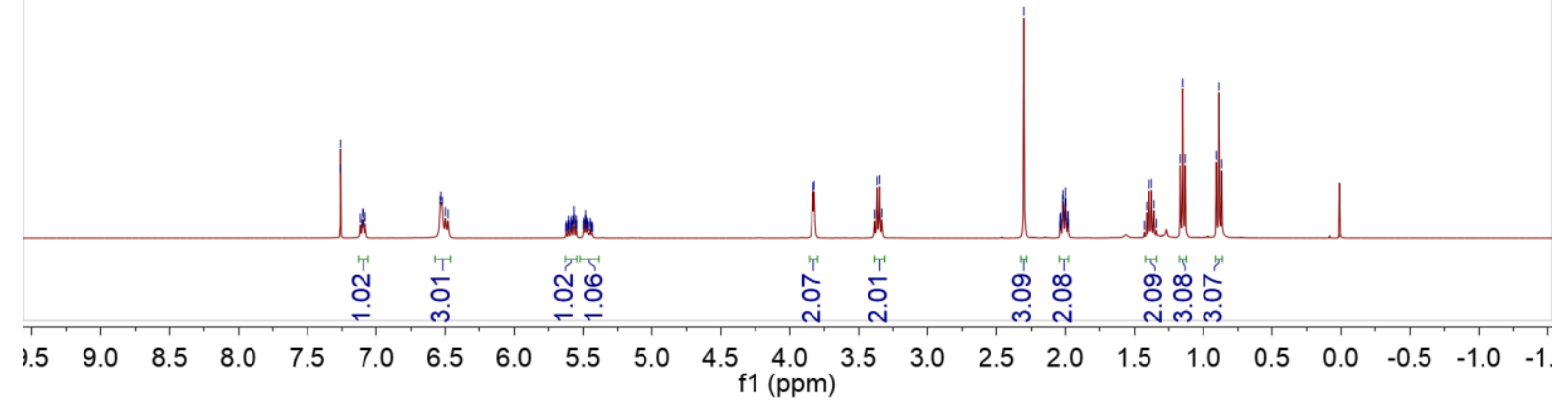

L

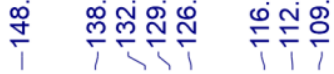

${ }^{13} \mathrm{C}$ NMR $\left(100 \mathrm{MHz}, \mathrm{CDCl}_{3}\right)$<smiles>CCC/C=C/CN(CC)c1cccc(C)c1</smiles>

10

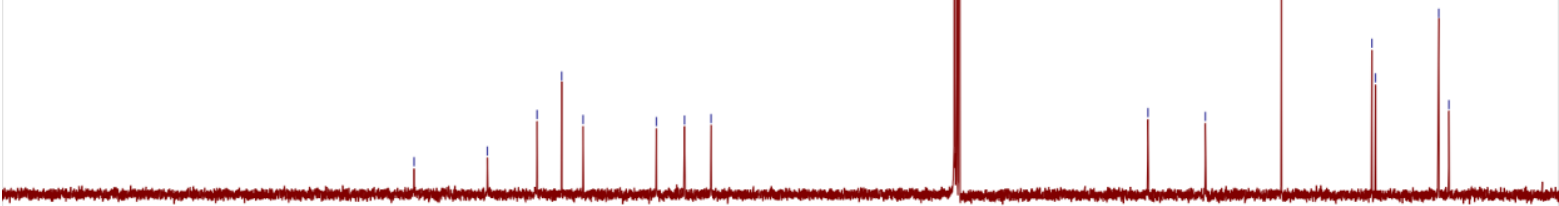




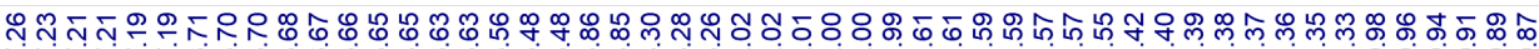

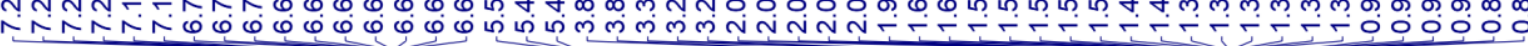

${ }^{1} \mathrm{H}$ NMR $\left(400 \mathrm{MHz}, \mathrm{CDCl}_{3}\right)$<smiles>CCC/C=C/CN(CCCC)c1ccccc1</smiles>

$1 p$

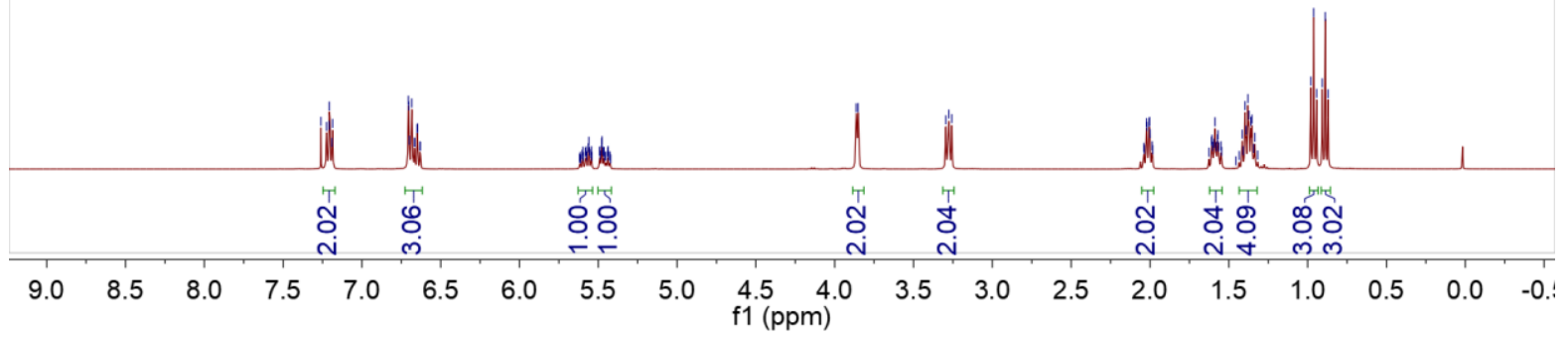

\begin{tabular}{|c|c|c|c|}
\hline 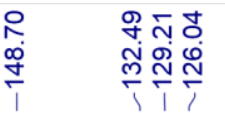 & 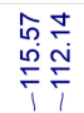 & 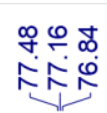 & 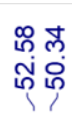 \\
\hline
\end{tabular}

${ }^{13} \mathrm{C}$ NMR $\left(100 \mathrm{MHz}, \mathrm{CDCl}_{3}\right)$<smiles>CCC/C=C/CN(CCCC)c1ccccc1</smiles>

$1 p$

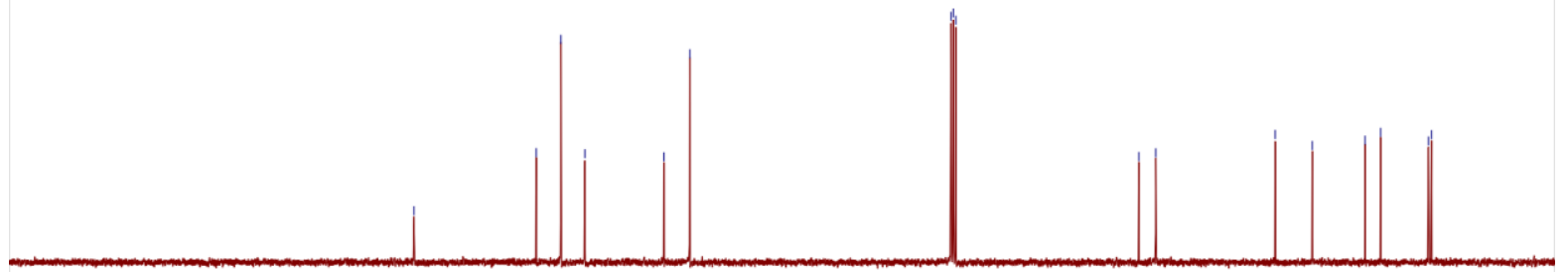

190180

160

$130 \quad 120$

110100

$\begin{array}{lllllllll}80 & 70 & 60 & 50 & 40 & 30 & 20 & 10 & 0\end{array}$ 


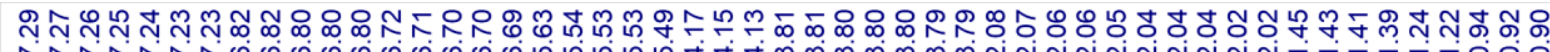

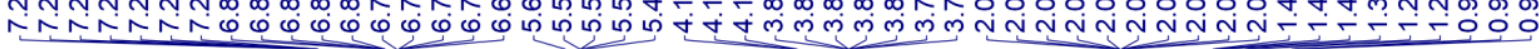

${ }^{1} \mathrm{H} \mathrm{NMR}\left(400 \mathrm{MHz}, \mathrm{CDCl}_{3}\right)$<smiles>CCC/C=C/CN(CCC)c1ccccc1</smiles>

19

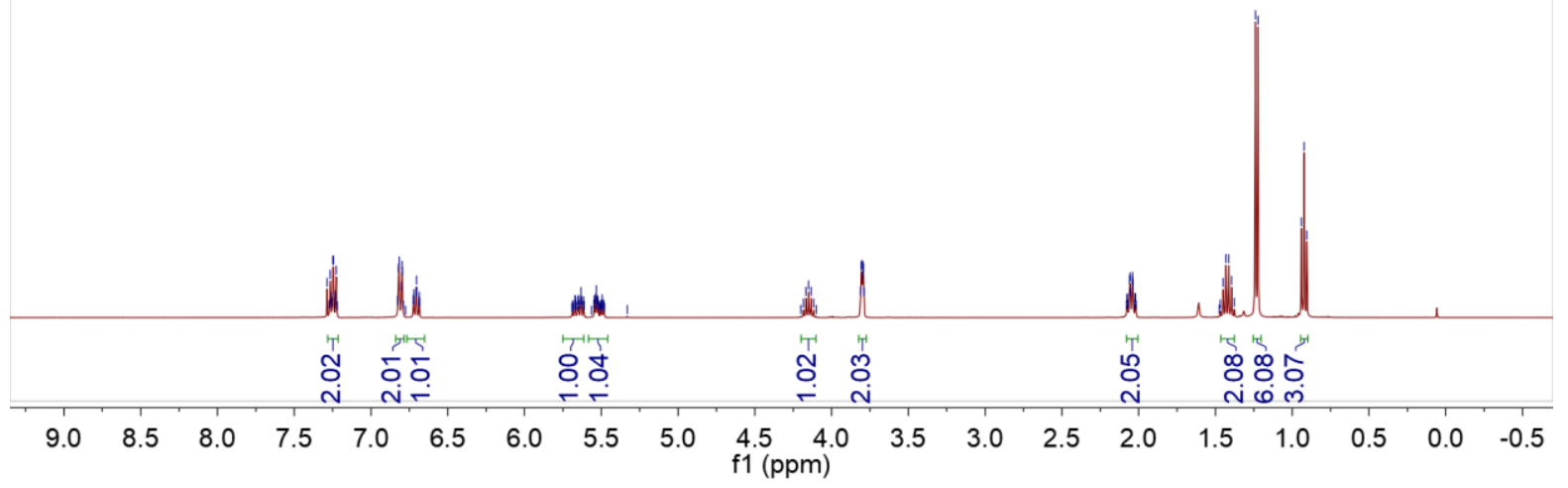

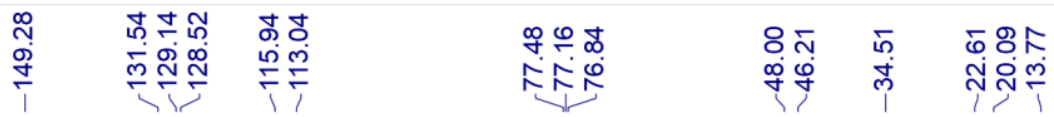<smiles>CCC/C=C\CN(CCC)c1ccccc1</smiles>

$1 q$

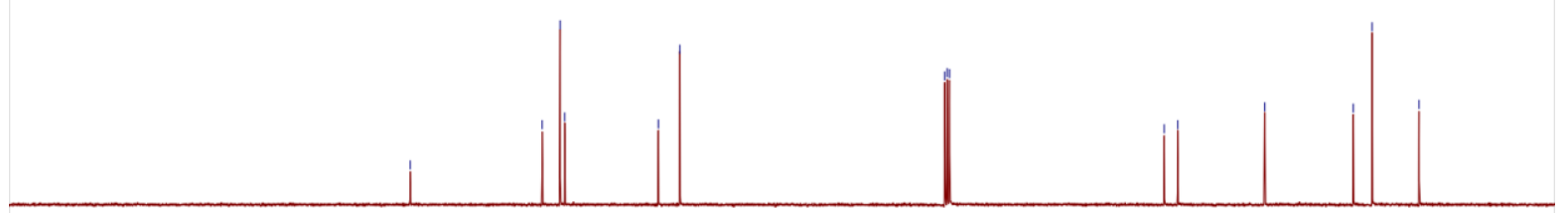


${ }^{1} \mathrm{H}$ NMR $\left(400 \mathrm{MHz}, \mathrm{CDCl}_{3}\right)$<smiles>CCC/C=C/CN(Cc1ccccc1)c1ccccc1</smiles>

$1 \mathrm{r}$

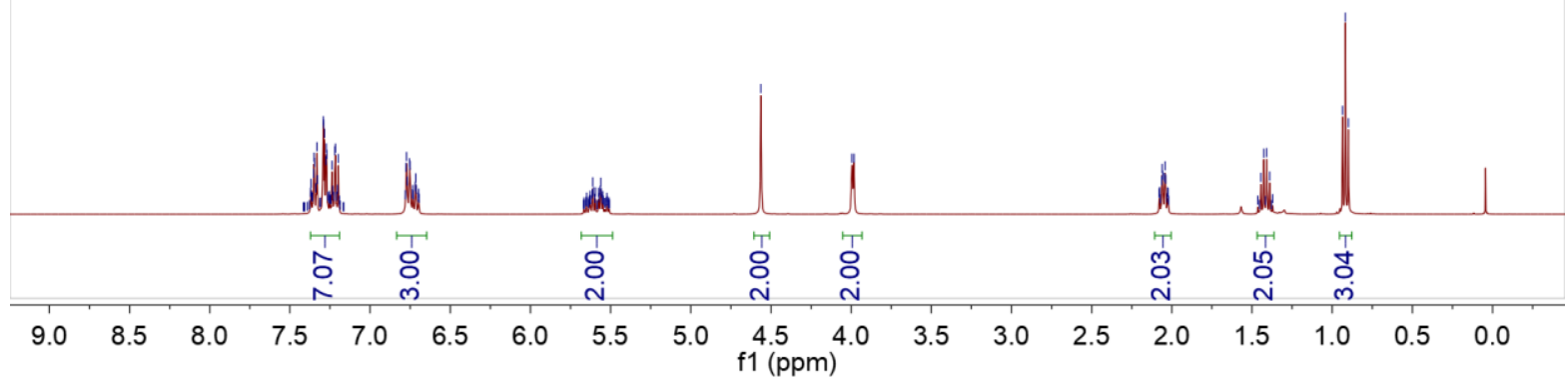

กุ จฺุ

守

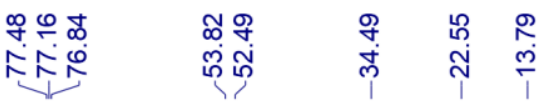

${ }^{13} \mathrm{C}$ NMR $\left(100 \mathrm{MHz}, \mathrm{CDCl}_{3}\right)$

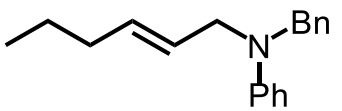

$1 \mathrm{r}$

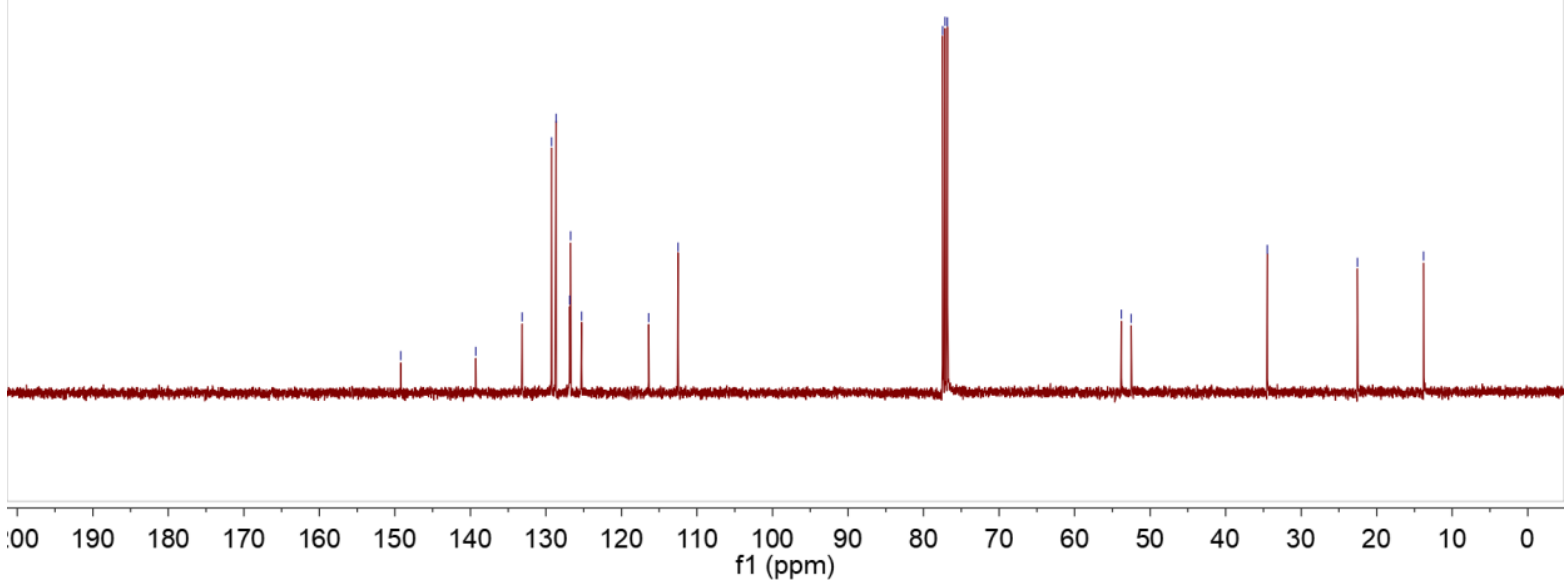


${ }^{1} \mathrm{H}$ NMR $\left(400 \mathrm{MHz}, \mathrm{CDCl}_{3}\right)$<smiles>CCC/C=C/CN(C)c1ccc(C)cc1</smiles>

$1 \mathrm{~s}$

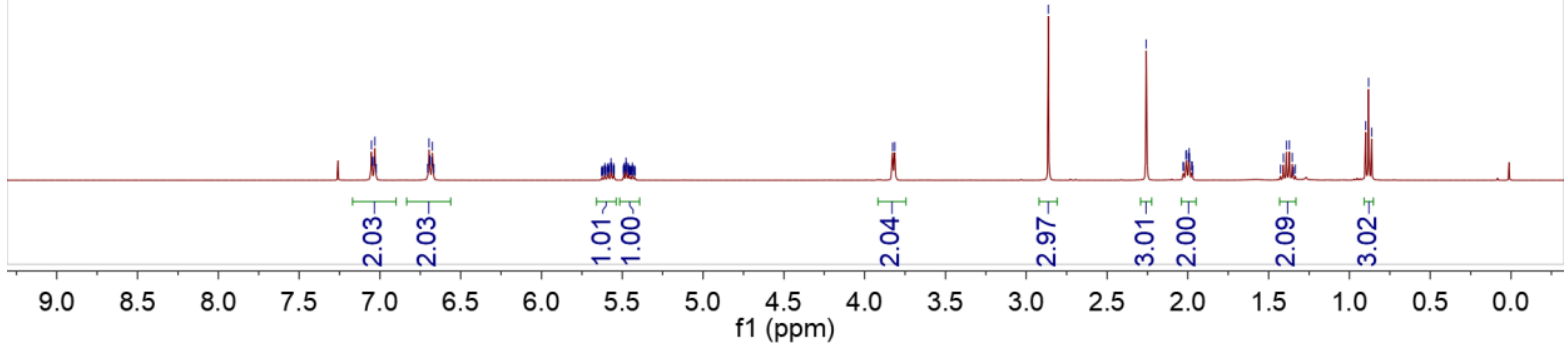

立

${ }^{13} \mathrm{C}$ NMR $\left(100 \mathrm{MHz}, \mathrm{CDCl}_{3}\right)$<smiles>CCC/C=C/CN(C)c1ccc(C)cc1</smiles>

$1 \mathrm{~s}$

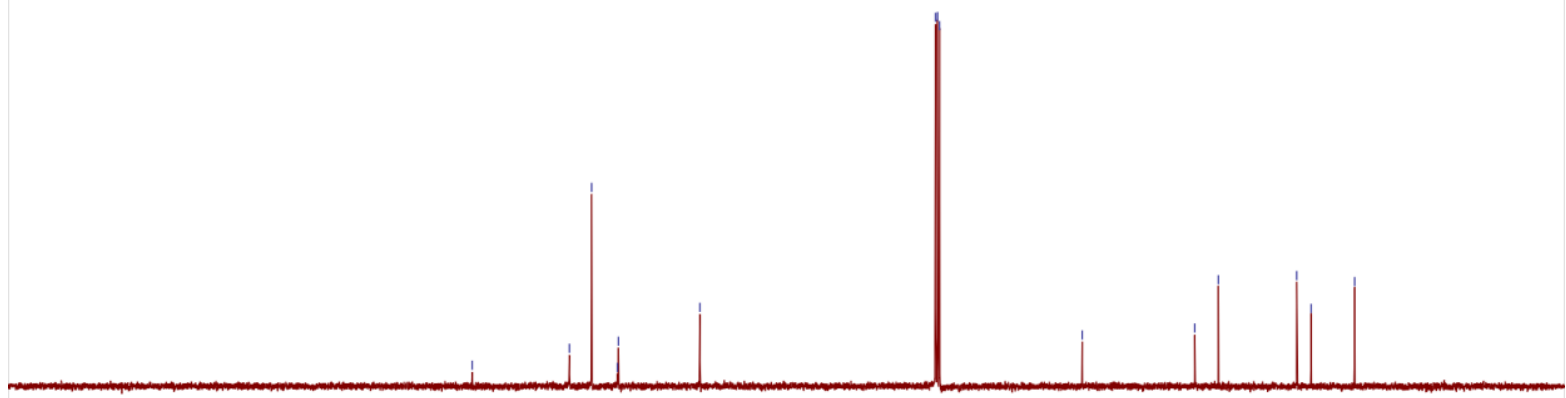

$\begin{array}{lllllllllllllllllllllll}210 & 200 & 190 & 180 & 170 & 160 & 150 & 140 & 130 & 120 & \begin{array}{c}110 \\ \mathrm{f} 1(\mathrm{ppm})\end{array} & 100 & 80 & 80 & 70 & 60 & 50 & 40 & 30 & 20 & 10 & 0 & -10\end{array}$ 
${ }^{1} \mathrm{H} \mathrm{NMR}\left(400 \mathrm{MHz}, \mathrm{CDCl}_{3}\right)$<smiles>CCC/C=C/CN(C)c1cccc(C)c1</smiles>

$1 \mathrm{t}$

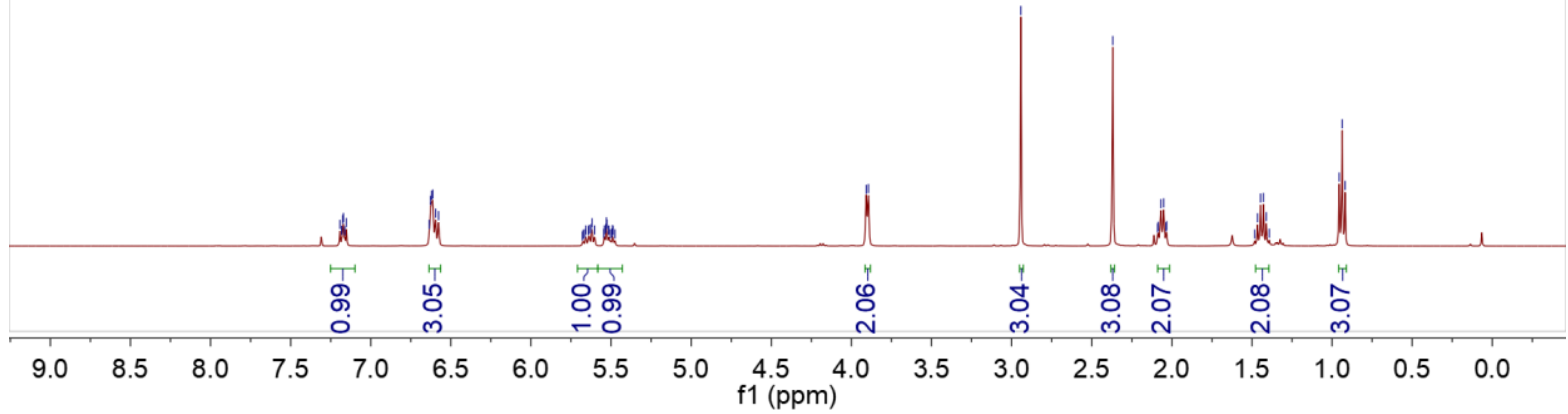

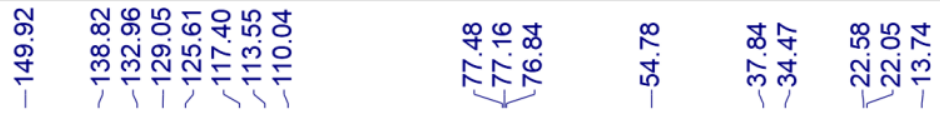

${ }^{13} \mathrm{C}$ NMR $\left(100 \mathrm{MHz}, \mathrm{CDCl}_{3}\right)$<smiles>CCC/C=C/CN(C)c1cccc(C)c1</smiles>

$1 \mathrm{t}$

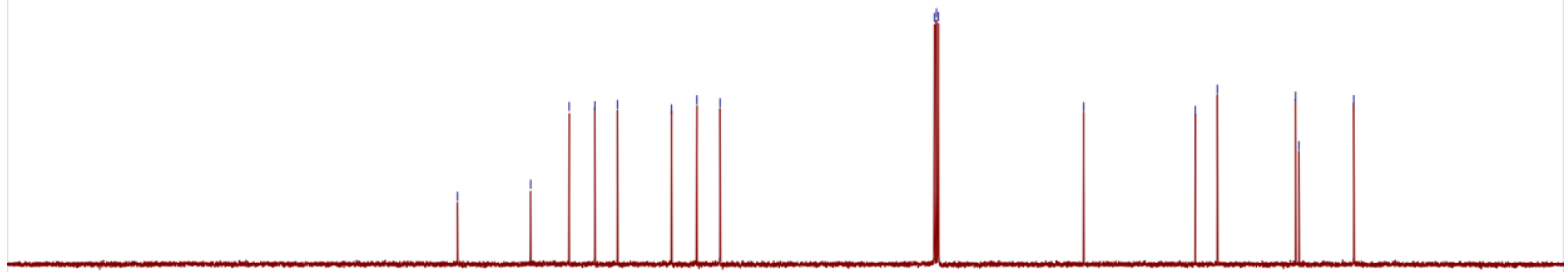

$\begin{array}{lllllllllllllllllllllll}210 & 200 & 190 & 180 & 170 & 160 & 150 & 140 & 130 & 120 & 110 \quad \begin{array}{l}100 \\ \mathrm{f} 1(\mathrm{ppm})\end{array} & 90 & 80 & 70 & 60 & 50 & 40 & 30 & 20 & 10 & 0 & -10\end{array}$ 


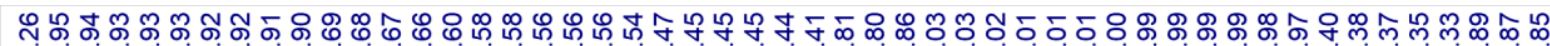

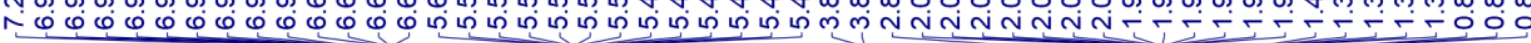

${ }^{1} \mathrm{H}$ NMR $\left(400 \mathrm{MHz}, \mathrm{CDCl}_{3}\right)$<smiles>CCC/C=C/CN(C)c1ccc(F)cc1</smiles>

1v

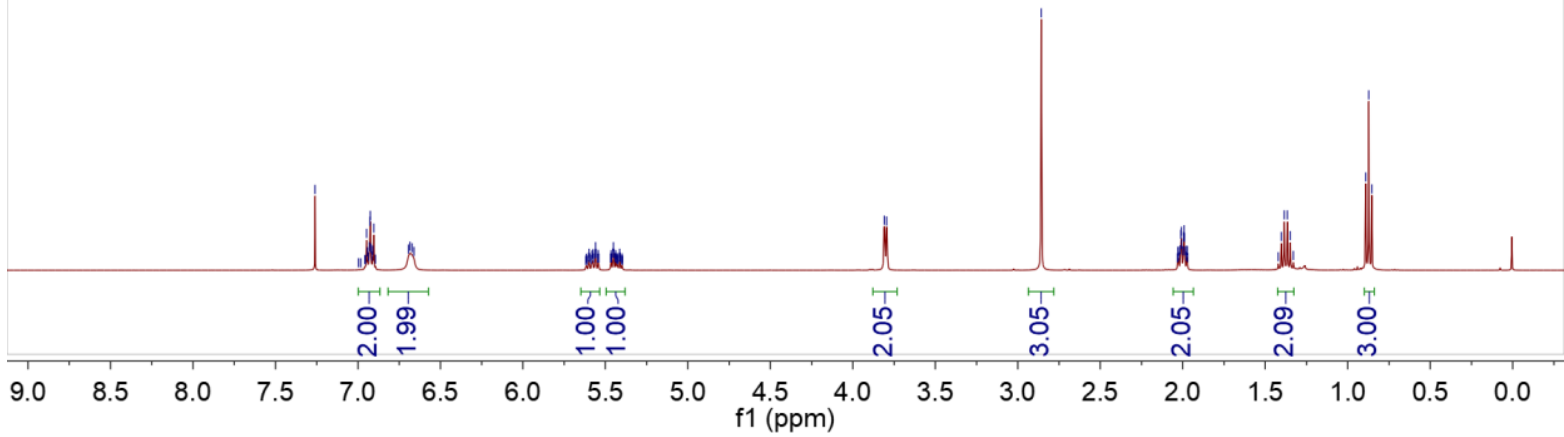

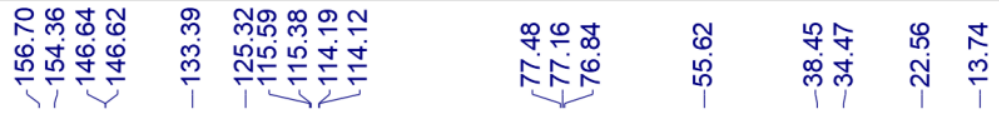

${ }^{13} \mathrm{C}$ NMR $\left(100 \mathrm{MHz}, \mathrm{CDCl}_{3}\right)$<smiles>CCC/C=C/CN(C)c1ccc(F)cc1</smiles>

$1 v$

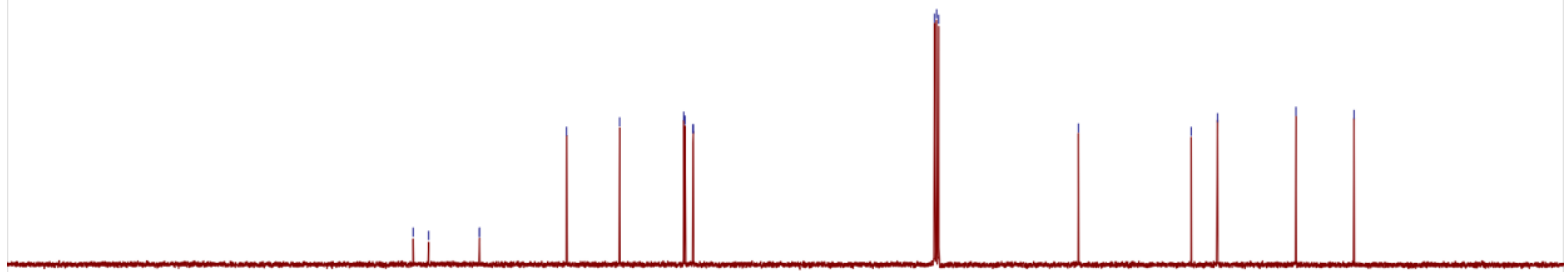

$\begin{array}{lllllllllllllllllllllll}210 & 200 & 190 & 180 & 170 & 160 & 150 & 140 & 130 & 120 & 110 \quad \begin{array}{l}100 \\ \mathrm{f} 1(\mathrm{ppm})\end{array} & 90 & 80 & 70 & 60 & 50 & 40 & 30 & 20 & 10 & 0 & -10\end{array}$ 
${ }^{19} \mathrm{~F} \mathrm{NMR}\left(377 \mathrm{MHz}, \mathrm{CDCl}_{3}\right)$<smiles>CCC/C=C/CN(C)c1ccc(F)cc1</smiles>

1v

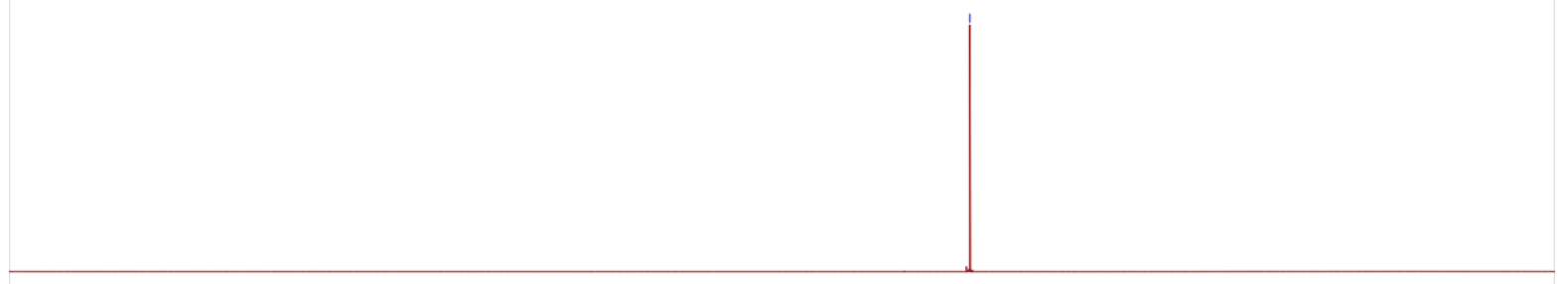

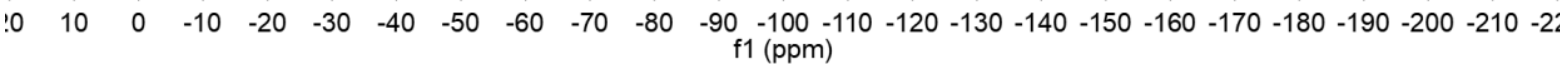

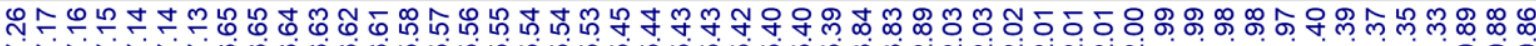

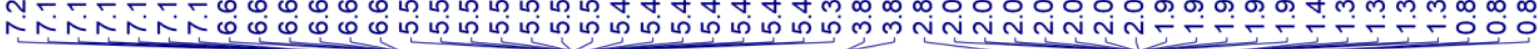

${ }^{1} \mathrm{H}$ NMR $\left(400 \mathrm{MHz}, \mathrm{CDCl}_{3}\right)$<smiles>CCC/C=C/CN(C)c1ccc(Cl)cc1</smiles>

$1 w$

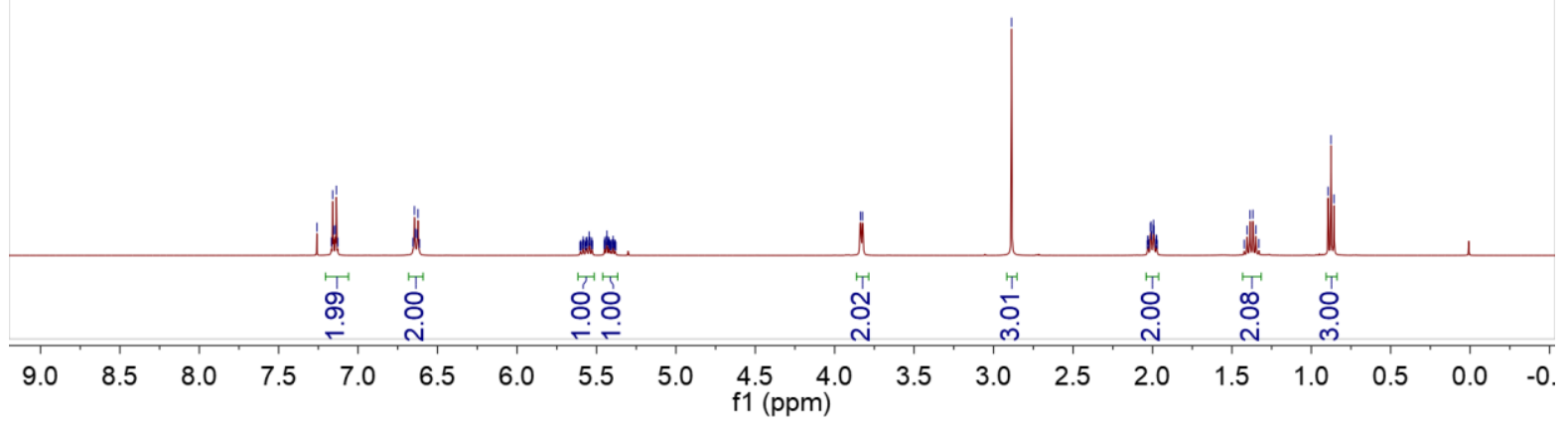




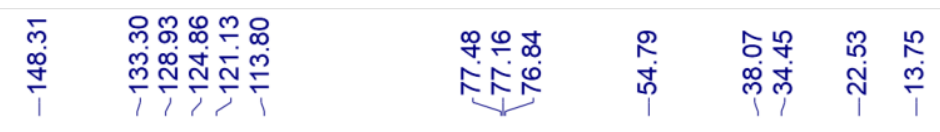

${ }^{13} \mathrm{C}$ NMR $\left(100 \mathrm{MHz}, \mathrm{CDCl}_{3}\right)$

$\overbrace{1}^{C l}$

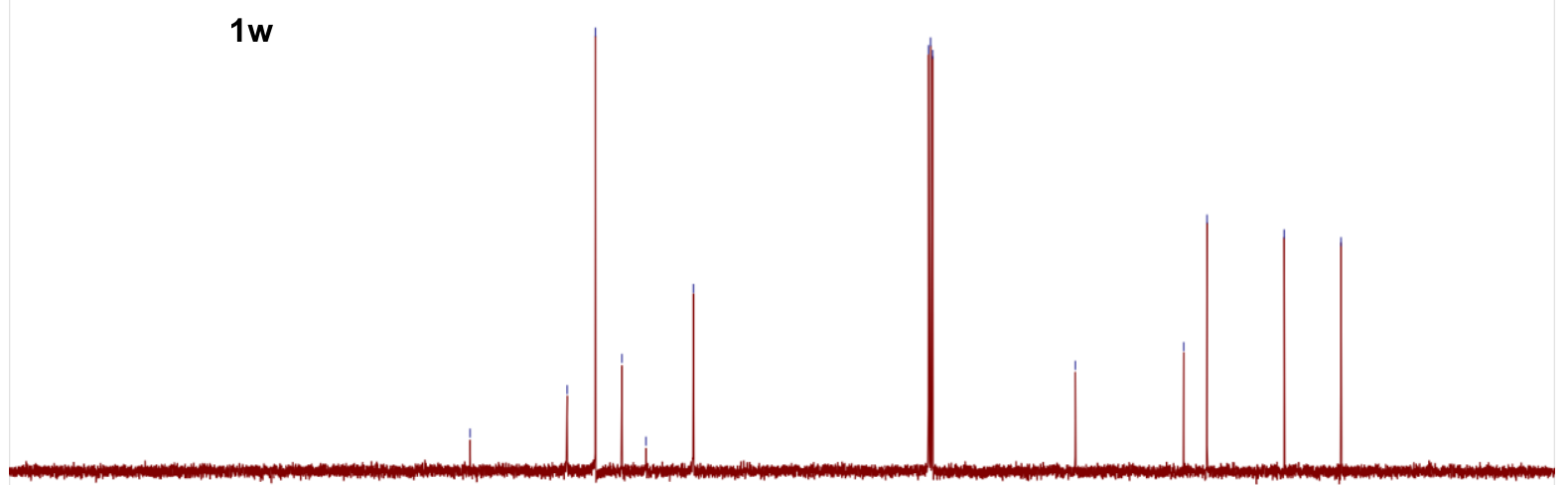

$\begin{array}{lllllllllllllllllllllll}210 & 200 & 190 & 180 & 170 & 160 & 150 & 140 & 130 & 120 & \begin{array}{c}110 \\ \mathrm{f} 1\end{array} \begin{array}{l}100 \\ (\mathrm{ppm})\end{array} & 90 & 80 & 70 & 60 & 50 & 40 & 30 & 20 & 10 & 0 & -10\end{array}$

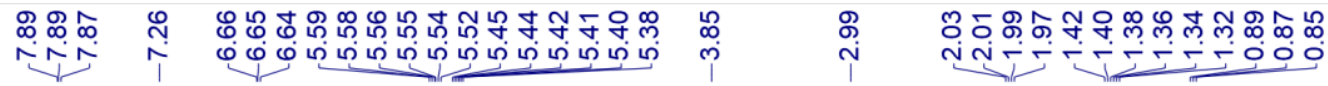

${ }^{1} \mathrm{H} \mathrm{NMR}\left(400 \mathrm{MHz}, \mathrm{CDCl}_{3}\right)$<smiles>[Y]C(=O)c1ccc(N(C)C/C=C/CCC)cc1</smiles>

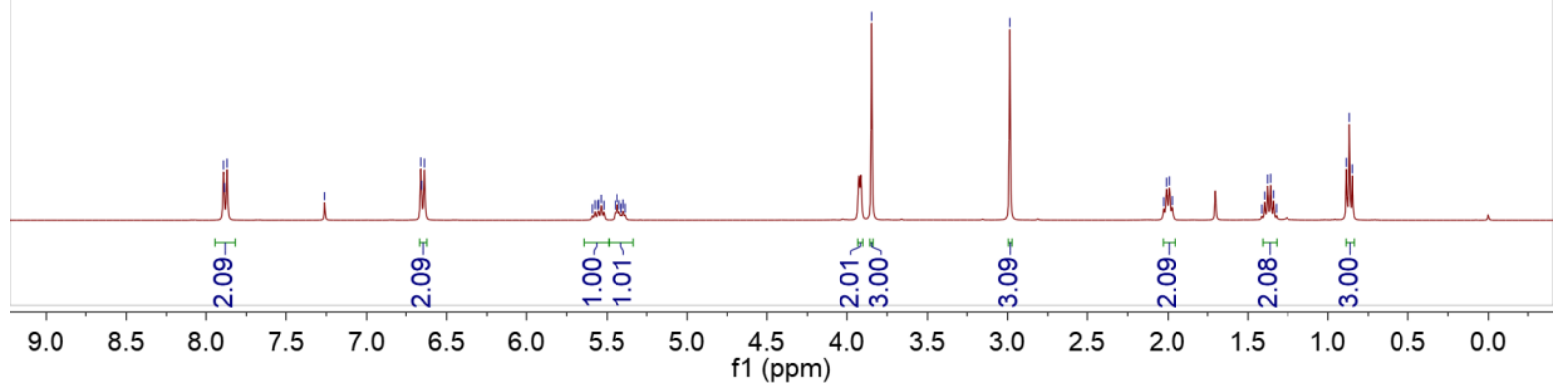




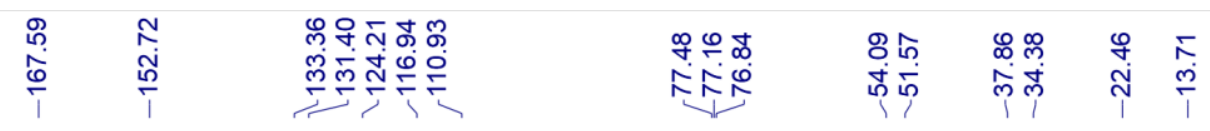

${ }^{13} \mathrm{C}$ NMR $\left(100 \mathrm{MHz}, \mathrm{CDCl}_{3}\right)$<smiles>CCC/C=C/CN(C)c1ccc(C(=O)OC)cc1</smiles>

$1 x$

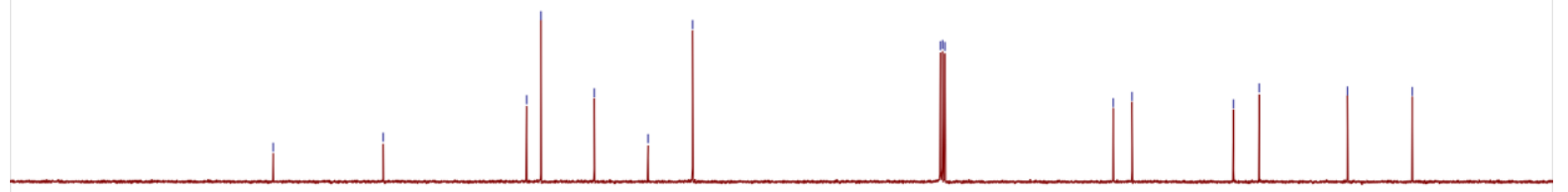

$\begin{array}{llllllllllllllllllll}200 & 190 & 180 & 170 & 160 & 150 & 140 & 130 & 120 & 110 \begin{array}{c}100 \\ \mathrm{f} 1(\mathrm{ppm})\end{array} & 90 & 80 & 70 & 60 & 50 & 40 & 30 & 20 & 10 & 0\end{array}$

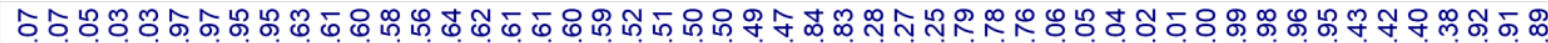

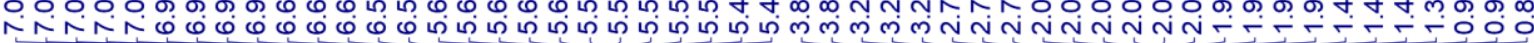

${ }^{1} \mathrm{H}$ NMR $\left(400 \mathrm{MHz}, \mathrm{CDCl}_{3}\right)$<smiles>CCC/C=C/CN1CCCc2ccccc21</smiles>

$1 y$

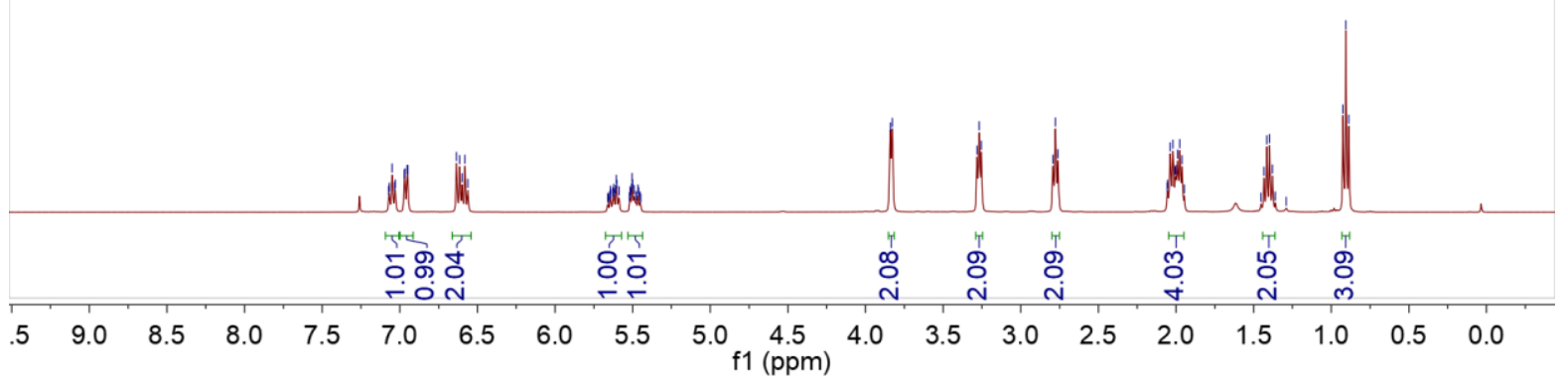




\begin{tabular}{|c|c|c|}
\hline 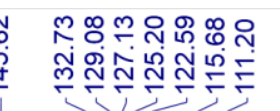 & 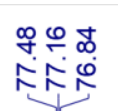 & 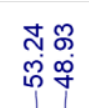 \\
\hline
\end{tabular}

${ }^{13} \mathrm{C}$ NMR $\left(100 \mathrm{MHz}, \mathrm{CDCl}_{3}\right)$<smiles>CCC/C=C/CN1CCCc2ccccc21</smiles>

1y

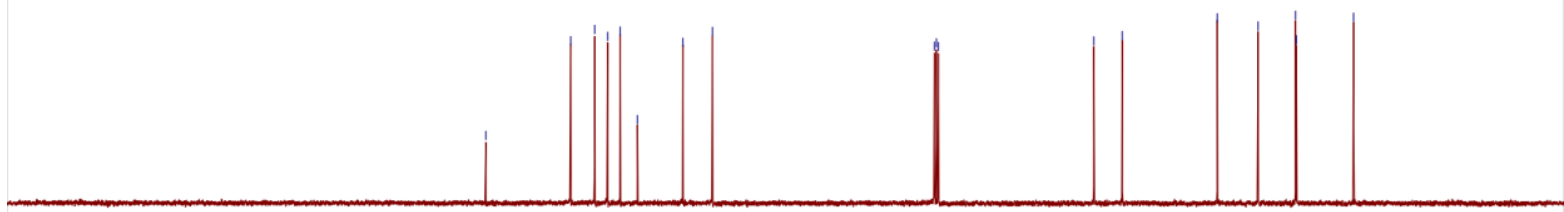

$\begin{array}{lllllllllllllllllllllll}210 & 200 & 190 & 180 & 170 & 160 & 150 & 140 & 130 & 120 & \begin{array}{c}110 \\ \mathrm{f} 1\end{array}\left(\begin{array}{ll}100 \\ (\mathrm{ppm})\end{array}\right. & 90 & 80 & 70 & 60 & 50 & 40 & 30 & 20 & 10 & 0 & -10\end{array}$

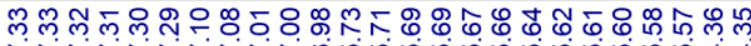

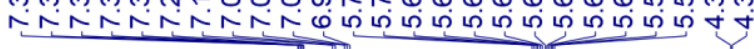

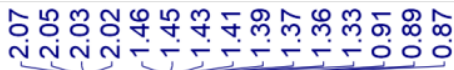<smiles>CCC/C=C/CN(CC(C)=O)c1ccccc1</smiles>

$1 z$

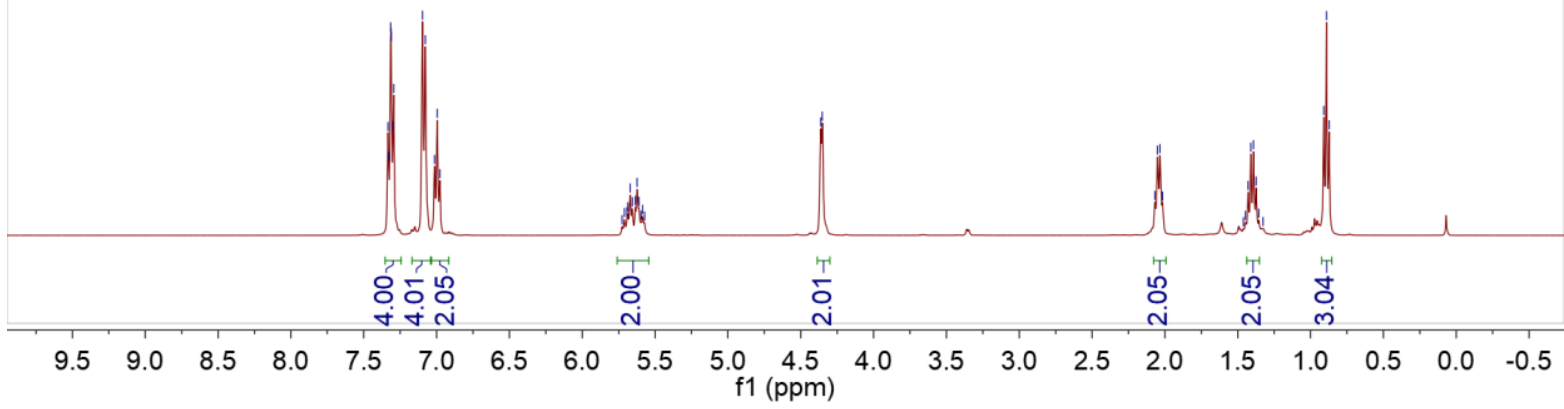




\begin{tabular}{|c|c|c|c|c|}
\hline 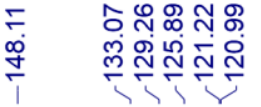 & 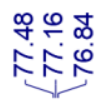 & $\begin{array}{l}\text { ○्ల } \\
\text { फं }\end{array}$ & $\stackrel{\substack{+\dot{m}}}{\dot{j}}$ & $\stackrel{\bar{n}}{\mathbb{N}}$ \\
\hline
\end{tabular}

${ }^{13} \mathrm{C}$ NMR $\left(100 \mathrm{MHz}, \mathrm{CDCl}_{3}\right)$<smiles>CCC/C=C/CN(c1ccccc1)c1ccccc1</smiles>

$1 z$

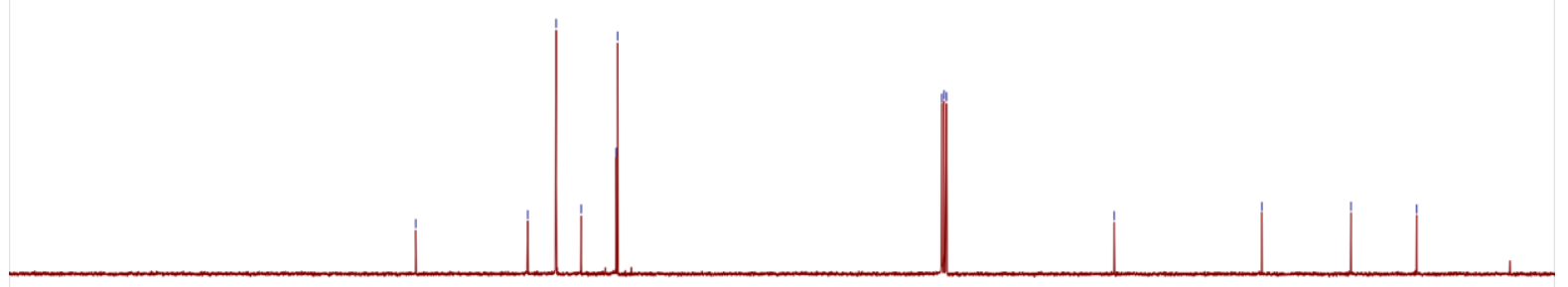

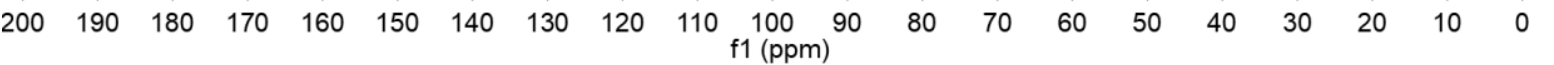

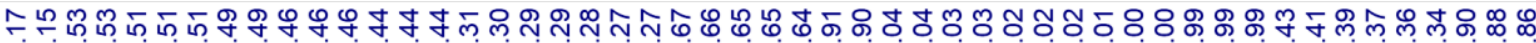

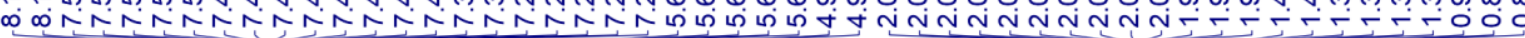

${ }^{1} \mathrm{H}$ NMR (100 MHz, $\mathrm{CDCl}_{3}$ )<smiles>CCC/C=C/Cn1c2ccccc2c2ccccc21</smiles>

1 aa

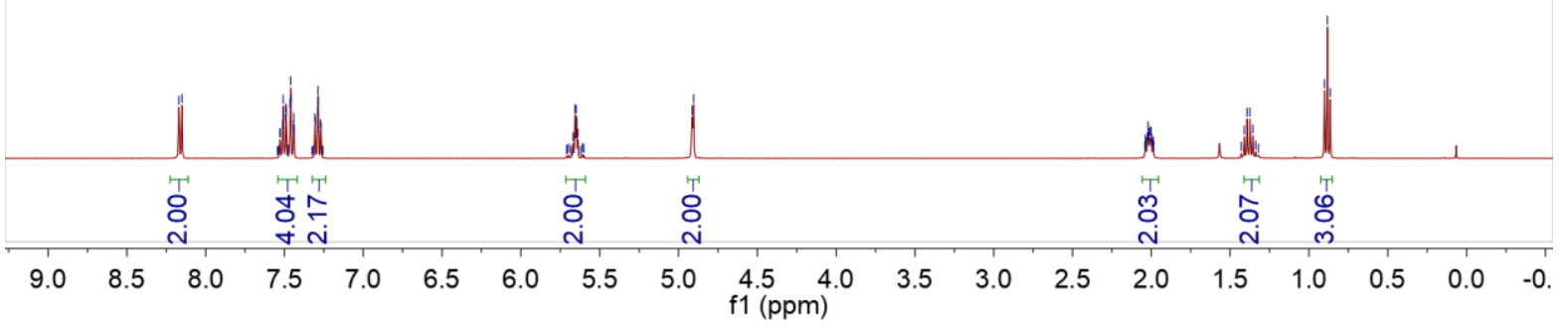




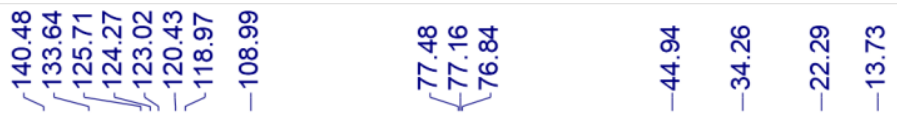

${ }^{13} \mathrm{C}$ NMR $\left(100 \mathrm{MHz}, \mathrm{CDCl}_{3}\right)$

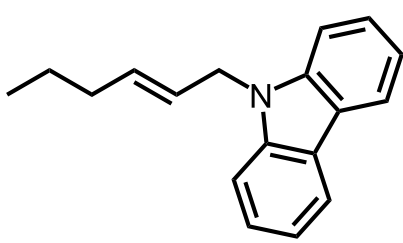

1 aa

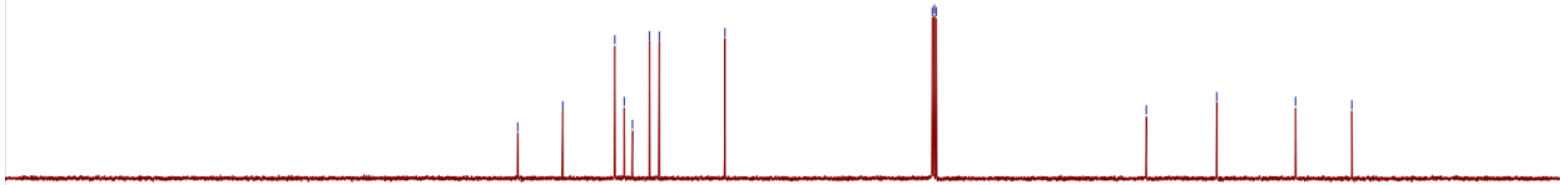

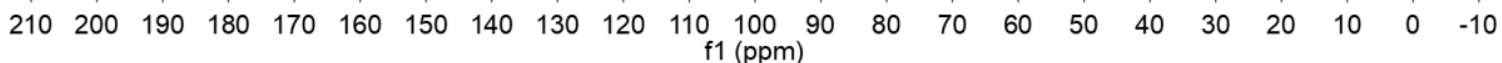

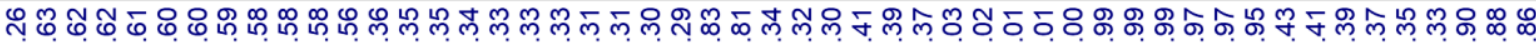

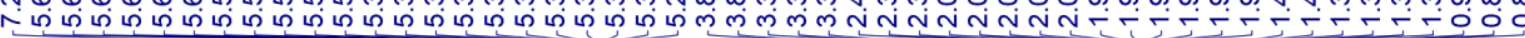

${ }^{1} \mathrm{H}$ NMR $\left(400 \mathrm{MHz}, \mathrm{CDCl}_{3}\right)$<smiles>CCC/C=C/CN1CCCC1=O</smiles>

$1 \mathrm{ab}$

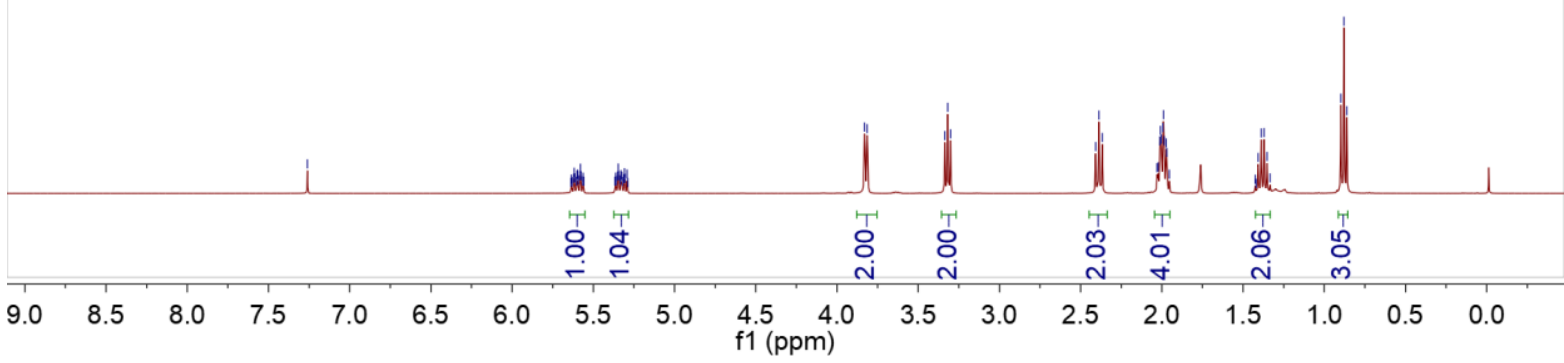




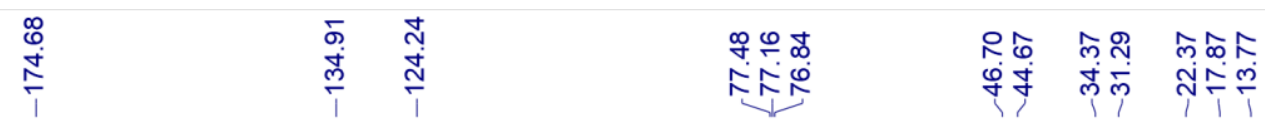

${ }^{13} \mathrm{C}$ NMR $\left(100 \mathrm{MHz}, \mathrm{CDCl}_{3}\right)$

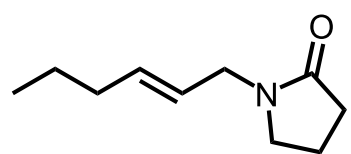

$1 a b$

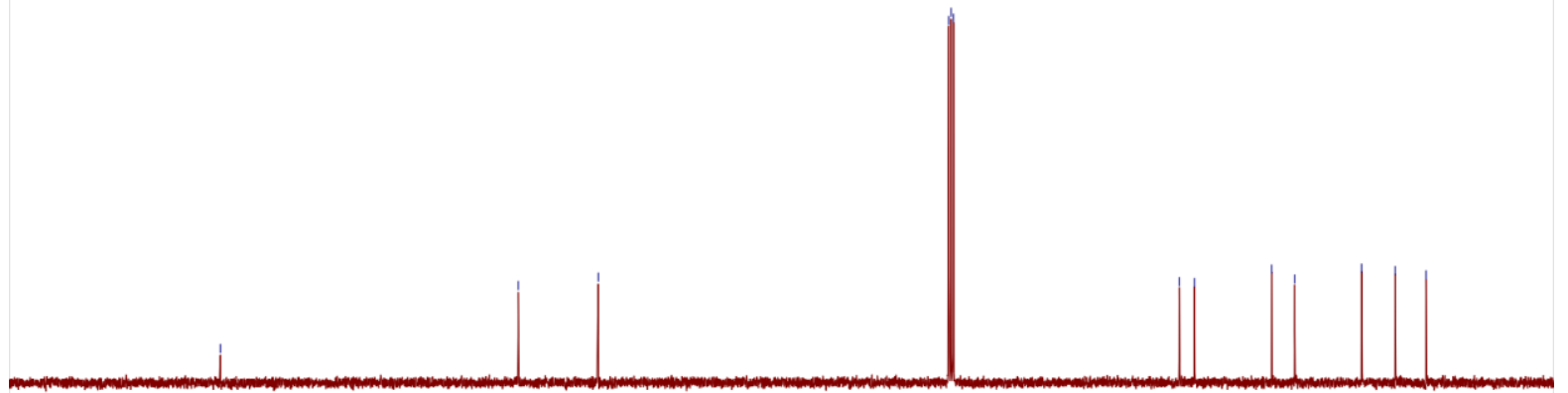

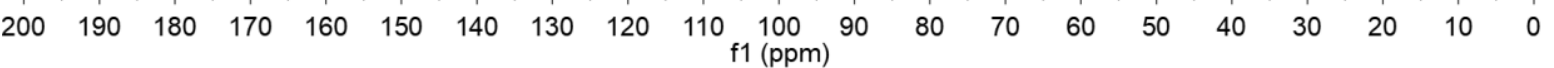

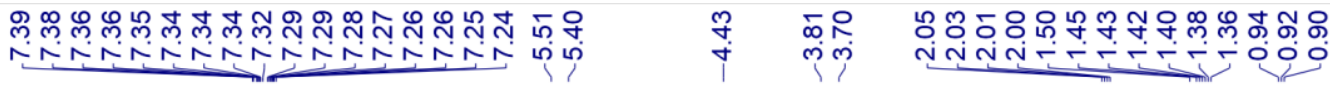

${ }^{1} \mathrm{H}$ NMR $\left(400 \mathrm{MHz}, \mathrm{CDCl}_{3}\right)$

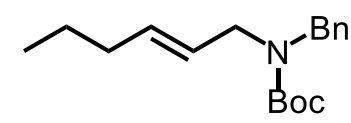

$1 \mathrm{ac}$

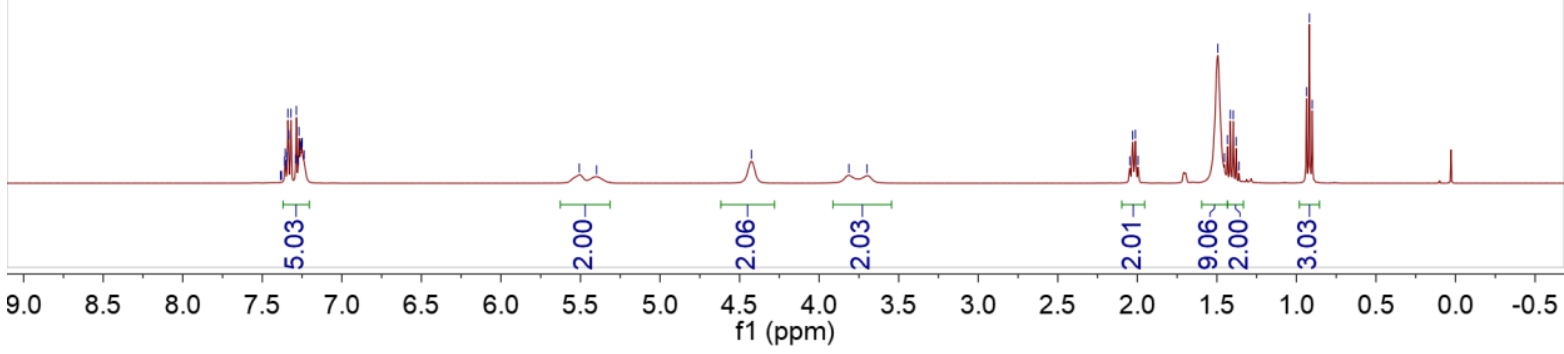




\begin{tabular}{|c|c|c|c|}
\hline 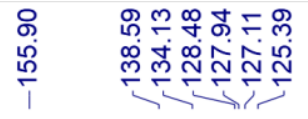 & 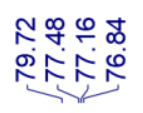 & 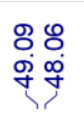 & 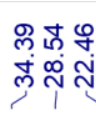 \\
\hline
\end{tabular}

${ }^{13} \mathrm{C}$ NMR $\left(100 \mathrm{MHz}, \mathrm{CDCl}_{3}\right)$

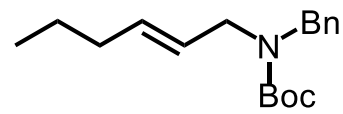

$1 \mathrm{ac}$

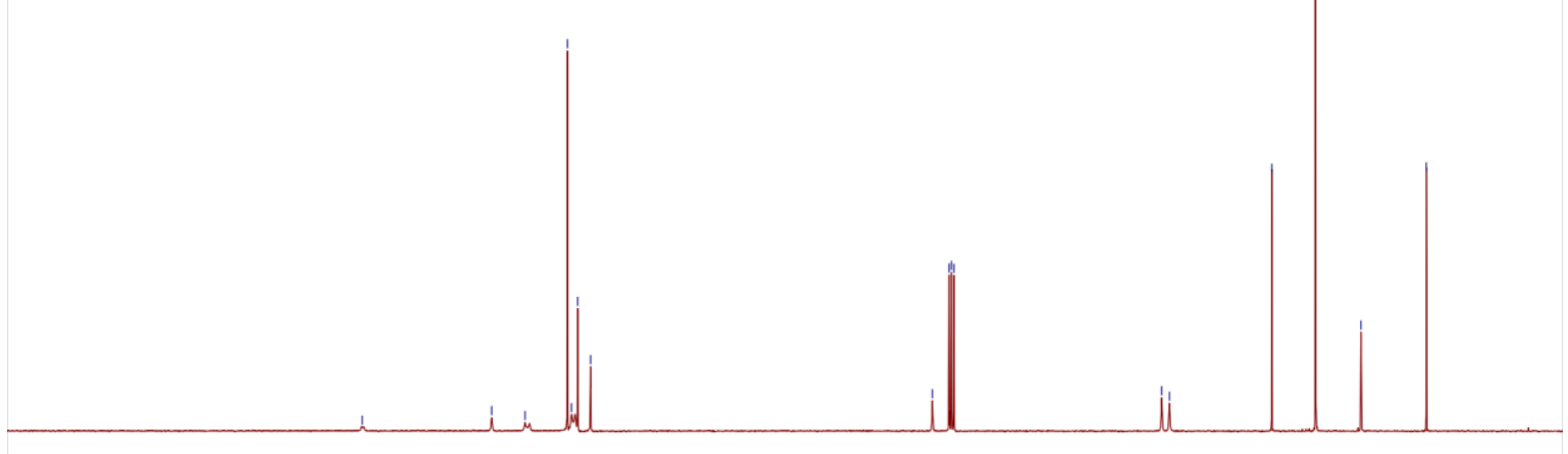

$\begin{array}{lllllllllllllllllllll}200 & 190 & 180 & 170 & 160 & 150 & 140 & 130 & 120 & 110 & \begin{array}{c}100 \\ \mathrm{f} 1(\mathrm{ppm})\end{array} & 90 & 80 & 70 & 60 & 50 & 40 & 30 & 20 & 10 & 0\end{array}$

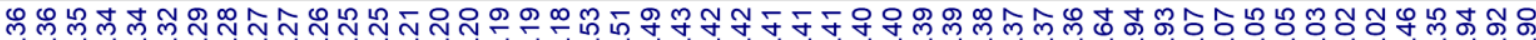

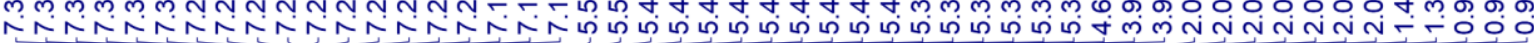

${ }^{1} \mathrm{H}$ NMR $\left(400 \mathrm{MHz}, \mathrm{CDCl}_{3}\right)$<smiles>CCC/C=C/CN(Br)Br</smiles>

$1 \mathrm{ad}$

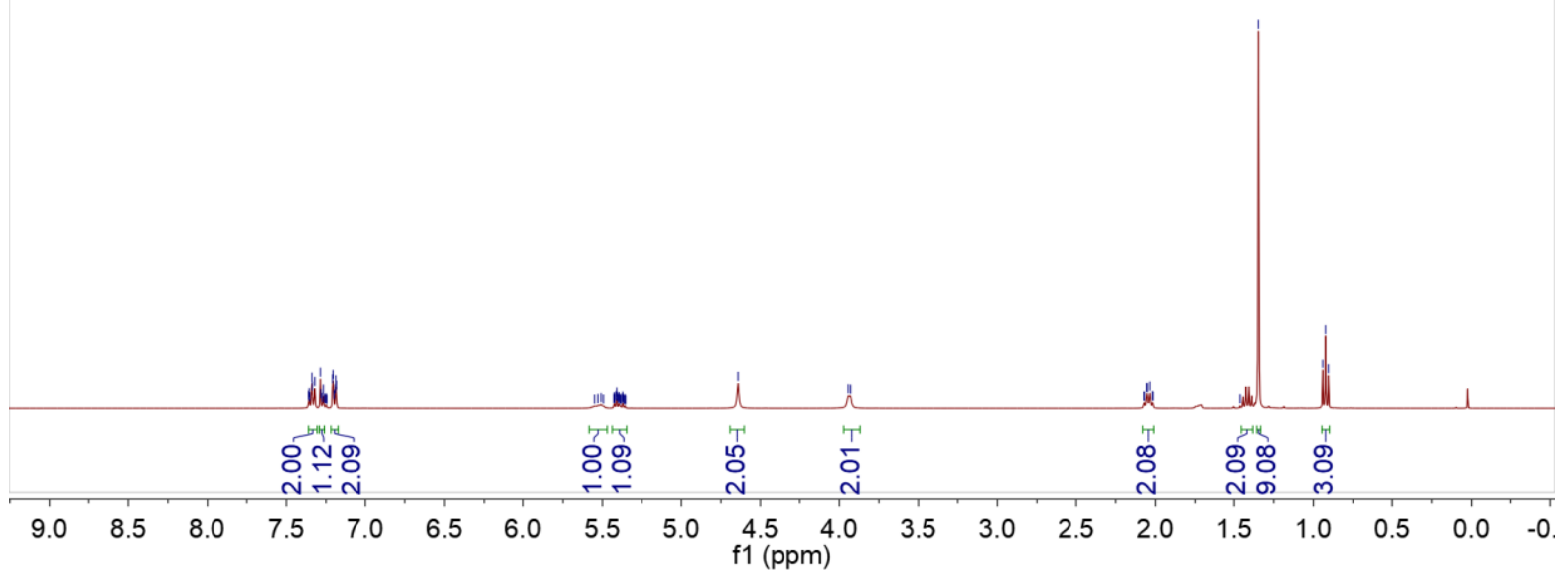




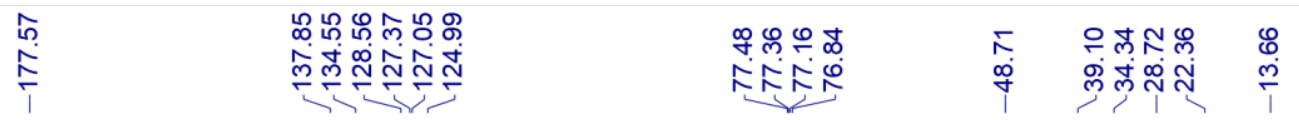

${ }^{13} \mathrm{C}$ NMR $\left(100 \mathrm{MHz}, \mathrm{CDCl}_{3}\right)$<smiles>CCC/C=C/CN(Cc1ccccc1)[PH](=O)(=O)c1ccccc1</smiles>

1 ad

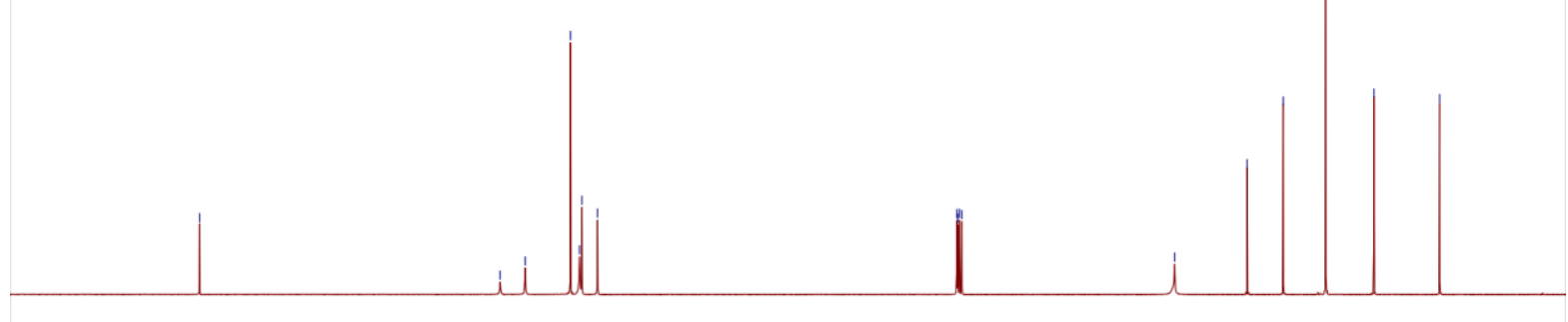

$\begin{array}{lllllllllllllllllllll}200 & 190 & 180 & 170 & 160 & 150 & 140 & 130 & 120 & 110 \underset{\mathrm{f} 1(\mathrm{ppm})}{100} & 90 & 80 & 70 & 60 & 50 & 40 & 30 & 20 & 10 & 0\end{array}$

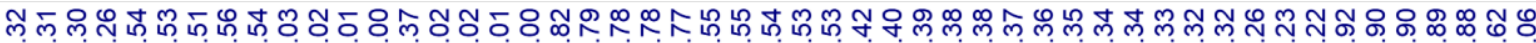

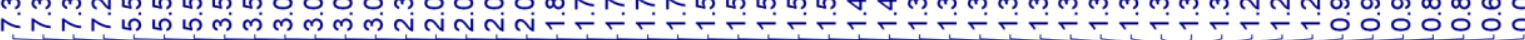

${ }^{1} \mathrm{H}$ NMR $\left(400 \mathrm{MHz}, \mathrm{CDCl}_{3}\right)$

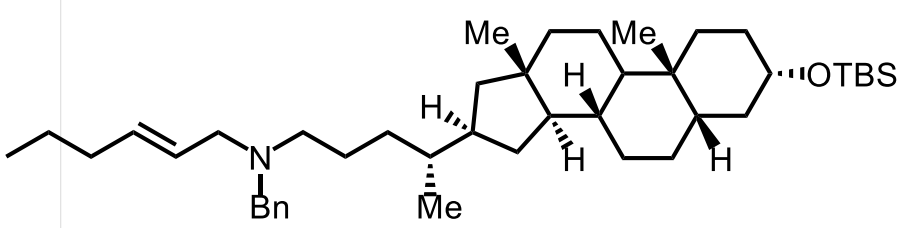

1 ae

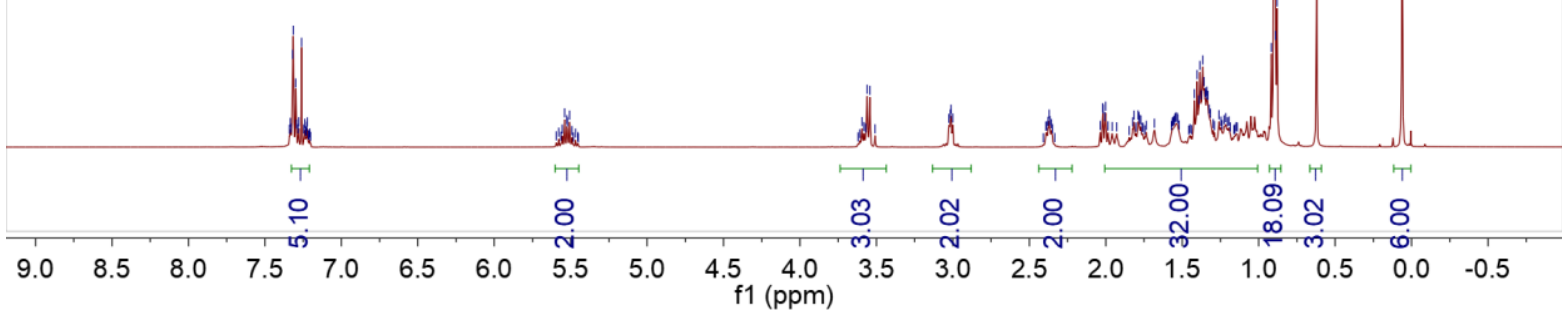


${ }^{13} \mathrm{C}$ NMR $\left(100 \mathrm{MHz}, \mathrm{CDCl}_{3}\right)$

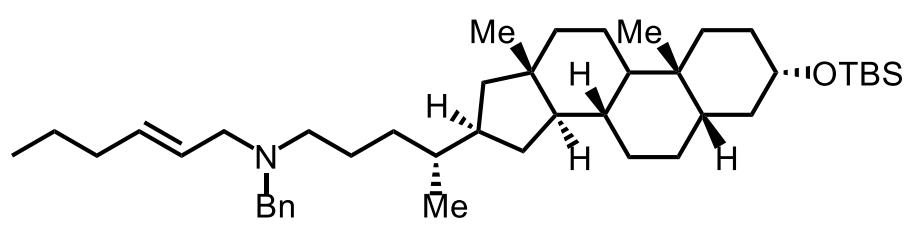

1 ae

${ }^{1} \mathrm{H}$ NMR $\left(400 \mathrm{MHz}, \mathrm{CDCl}_{3}\right)$<smiles>CCC/C=C/CN(Cc1ccccc1)CC(C)c1ccc(CC(C)C)cc1</smiles>

1 af

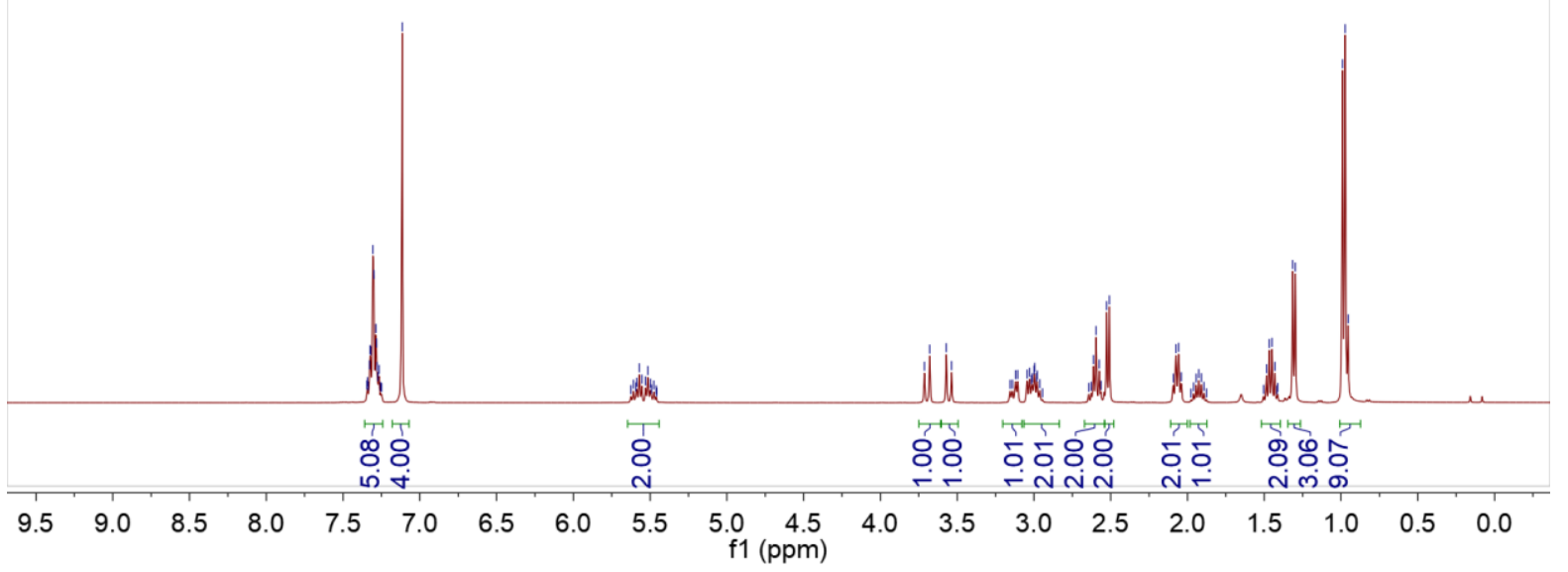


${ }^{13} \mathrm{C}$ NMR $\left(100 \mathrm{MHz}, \mathrm{CDCl}_{3}\right)$<smiles>CCC/C=C/CN(Cc1ccccc1)CC(C)c1ccc(CC(C)C)cc1</smiles>

1 af

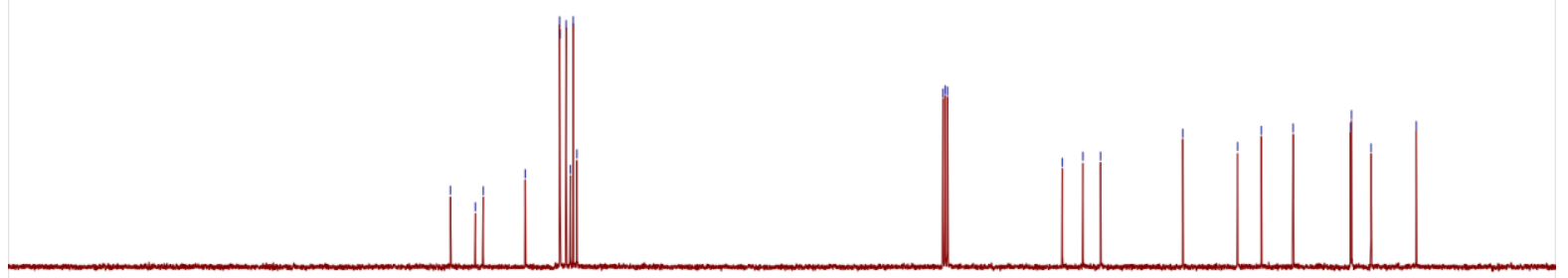

$\begin{array}{lllllllllllllllllllll}200 & 190 & 180 & 170 & 160 & 150 & 140 & 130 & 120 & 110 \underset{\mathrm{f} 1(\mathrm{ppm})}{100} 90 & 80 & 70 & 60 & 50 & 40 & 30 & 20 & 10 & 0\end{array}$

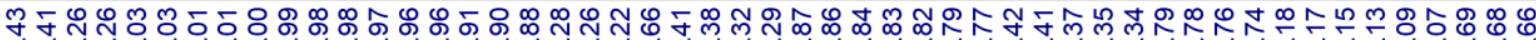

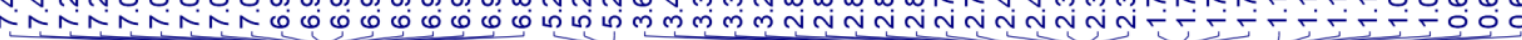

${ }^{1} \mathrm{H}$ NMR $\left(400 \mathrm{MHz}, \mathrm{CDCl}_{3}\right)$<smiles>CCC/C=C/CN(Cc1ccccc1)C[C@H](C)c1ccc2cc(OC)ccc2c1</smiles>

$1 \mathrm{ag}$

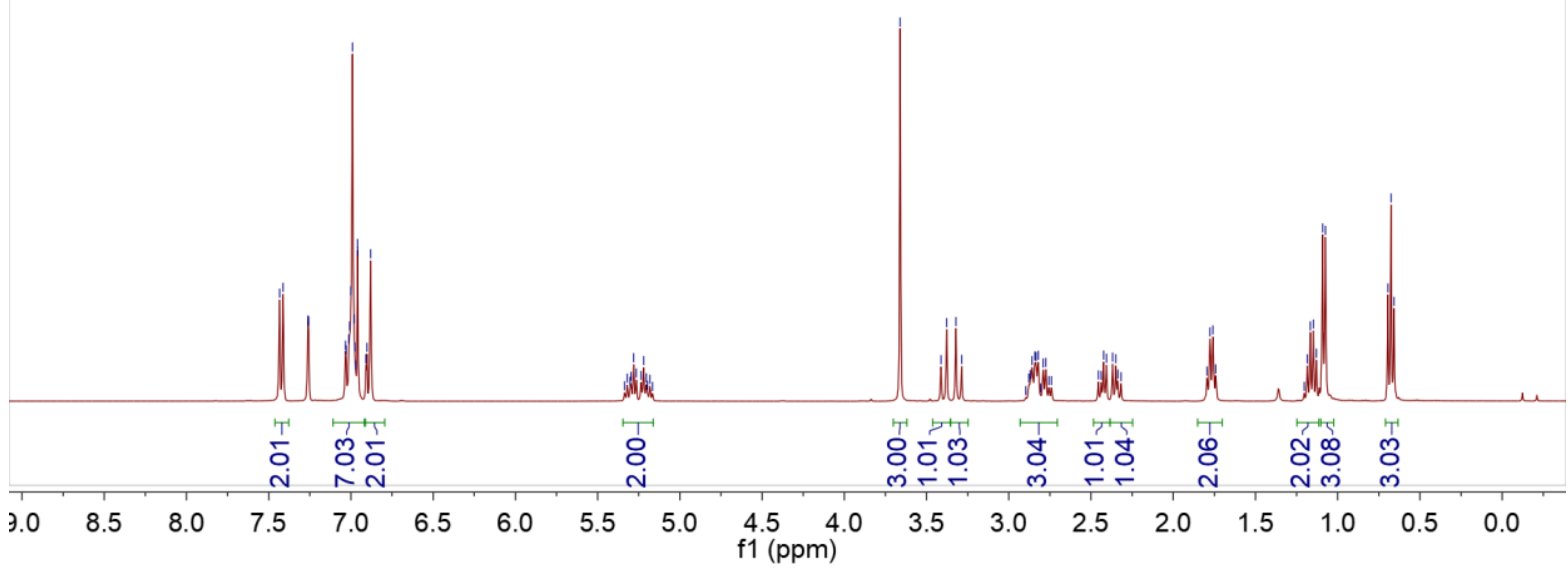


${ }^{13} \mathrm{C}$ NMR $\left(100 \mathrm{MHz}, \mathrm{CDCl}_{3}\right)$<smiles>CCC/C=C/CN(Cc1ccccc1)C[C@H](C)c1ccc2cc(OC)ccc2c1</smiles>

1 ag
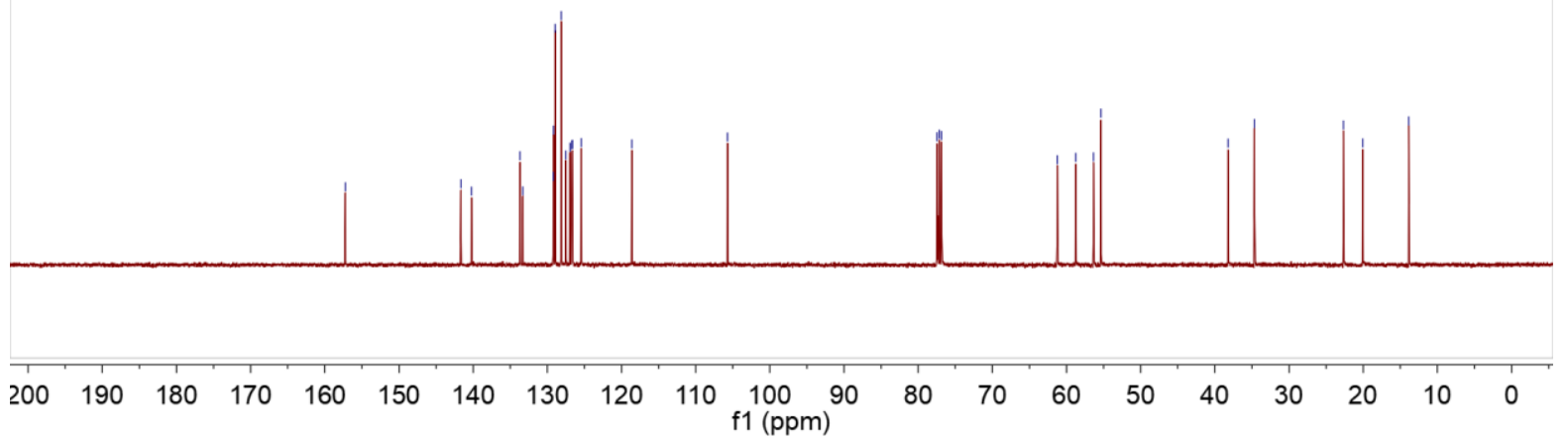

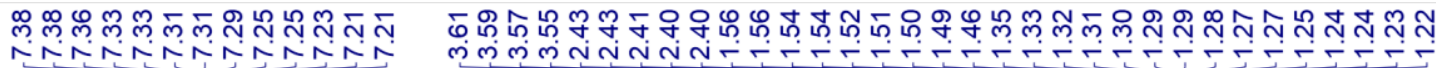

${ }^{1} \mathrm{H}$ NMR $\left(400 \mathrm{MHz}, \mathrm{CDCl}_{3}\right)$<smiles>OCCCCCCN(Cc1ccccc1)Cc1ccccc1</smiles>

$2 a$

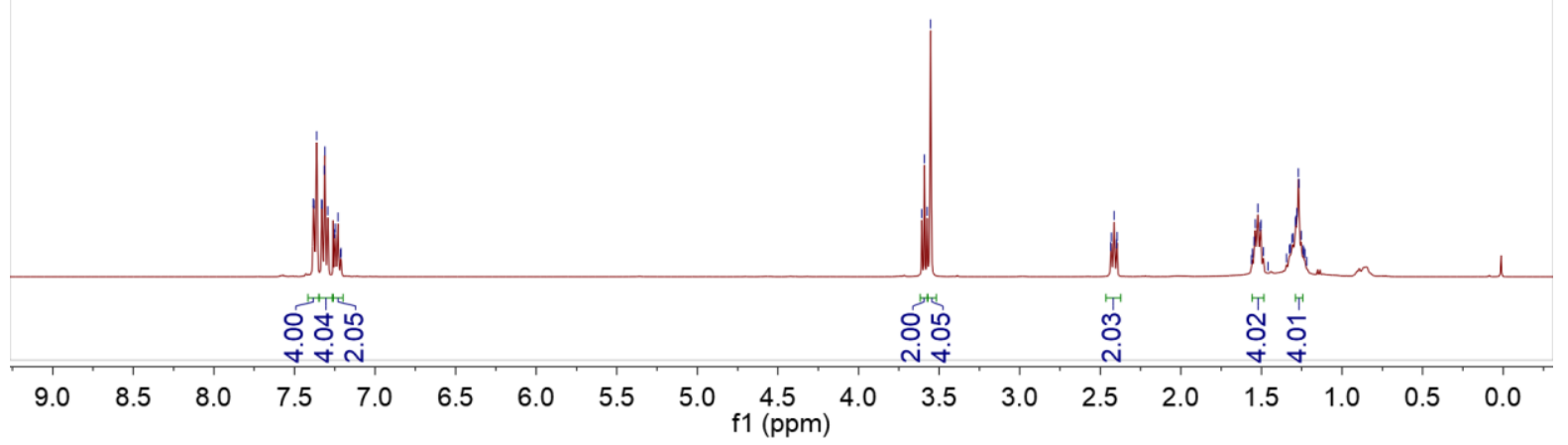




\section{অ

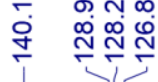

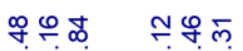

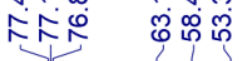

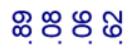 \\ กู่งิกั่}

${ }^{13} \mathrm{C}$ NMR $\left(100 \mathrm{MHz}, \mathrm{CDCl}_{3}\right)$

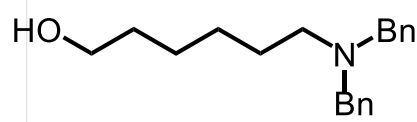

$2 a$

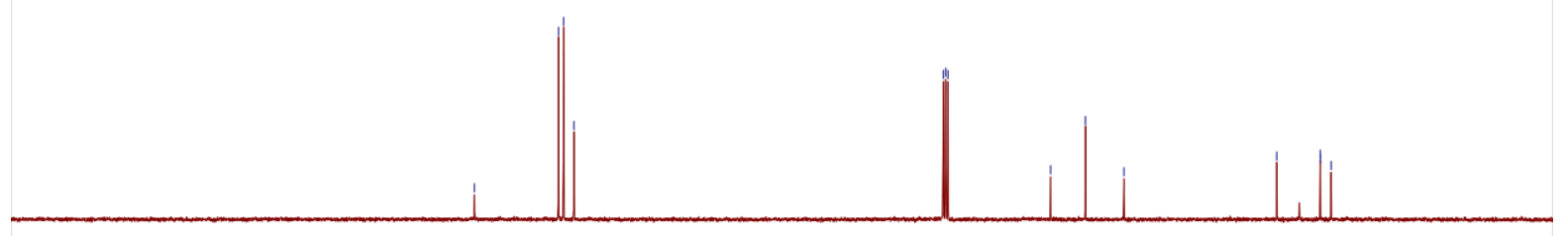

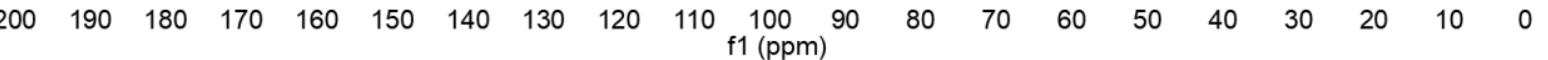

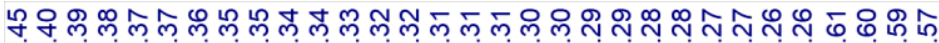

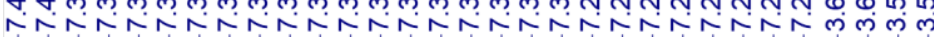

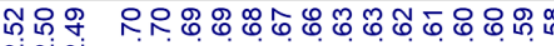

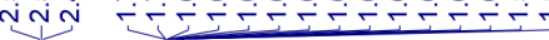

${ }^{1} \mathrm{H} \mathrm{NMR}\left(400 \mathrm{MHz}, \mathrm{CDCl}_{3}\right)$<smiles>OCCCCN(Cc1ccccc1)Cc1ccccc1</smiles>

2b

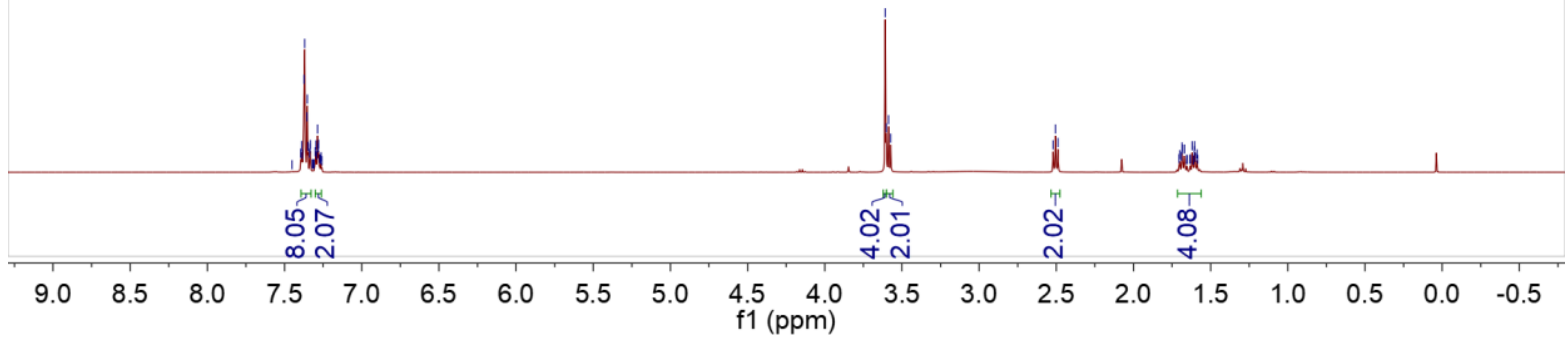


${ }^{13} \mathrm{C}$ NMR $\left(100 \mathrm{MHz}, \mathrm{CDCl}_{3}\right)$<smiles>OCCCCN(Cc1ccccc1)Cc1ccccc1</smiles>

2b

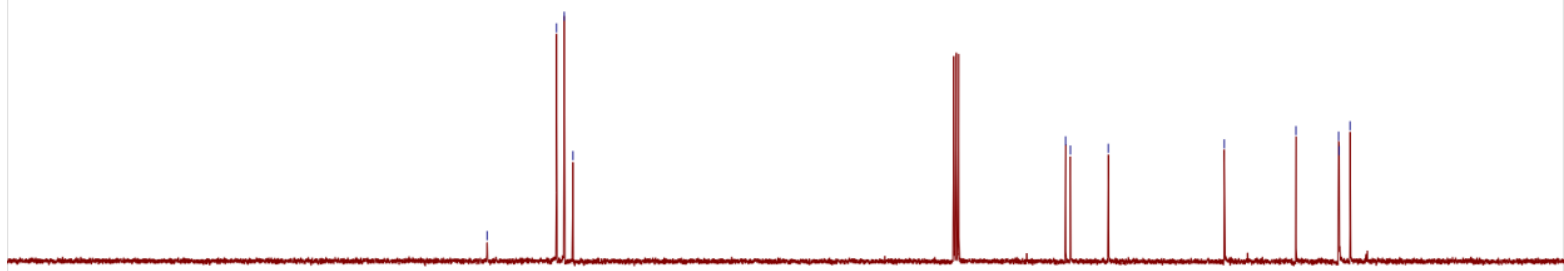

$\begin{array}{lllllllllllllllllllll}00 & 190 & 180 & 170 & 160 & 150 & 140 & 130 & 120 & 110 \underset{\mathrm{f} 1(\mathrm{ppm})}{100} & 90 & 80 & 70 & 60 & 50 & 40 & 30 & 20 & 10 & 0\end{array}$

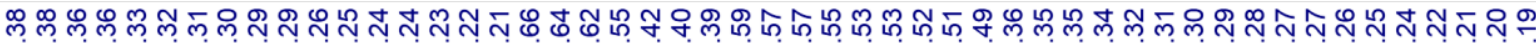

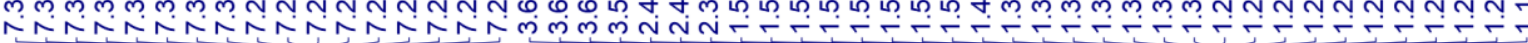

${ }^{1} \mathrm{H} \mathrm{NMR}\left(400 \mathrm{MHz}, \mathrm{CDCl}_{3}\right)$<smiles>OCCCCCCCCCCCN(Cc1ccccc1)Cc1ccccc1</smiles>

2c

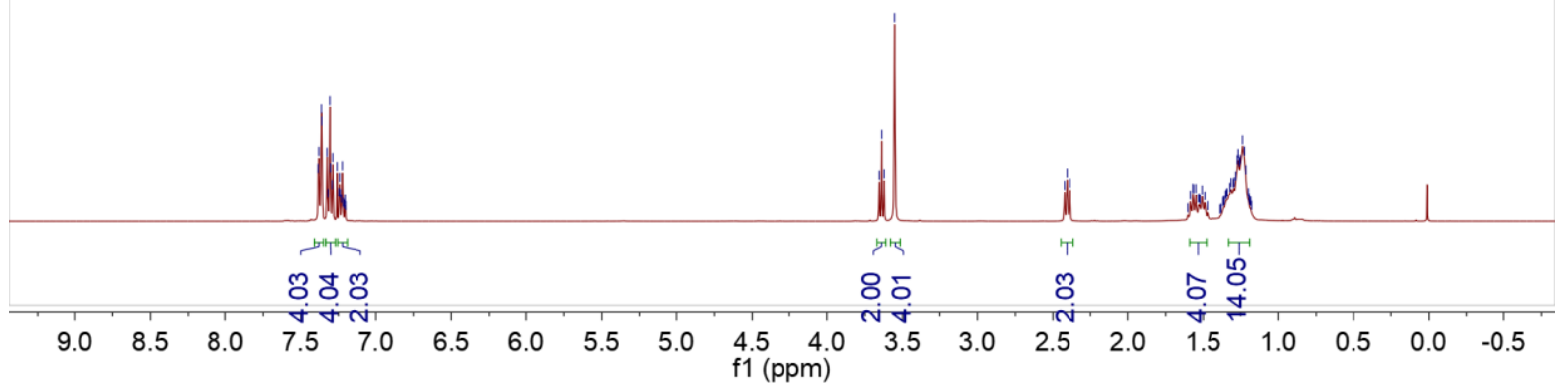




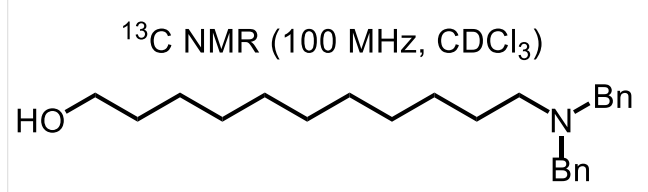

2c

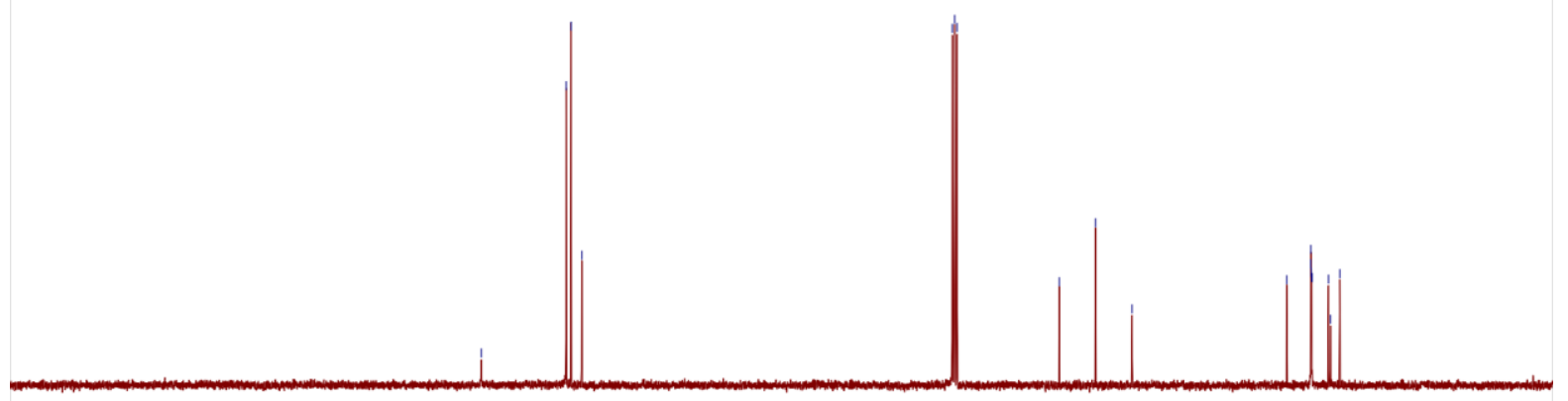

$\begin{array}{lllllllllllllllllllll}200 & 190 & 180 & 170 & 160 & 150 & 140 & 130 & 120 & 110 \begin{array}{c}100 \\ \mathrm{f} 1(\mathrm{ppm})\end{array} & 90 & 80 & 70 & 60 & 50 & 40 & 30 & 20 & 10 & 0\end{array}$

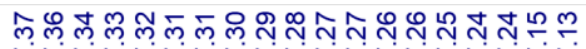

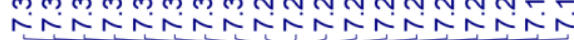

${ }^{1} \mathrm{H}$ NMR (400 MHz, $\mathrm{CDCl}_{3}$ )<smiles>CN(CCCCCCO)Cc1ccccc1</smiles>

2d

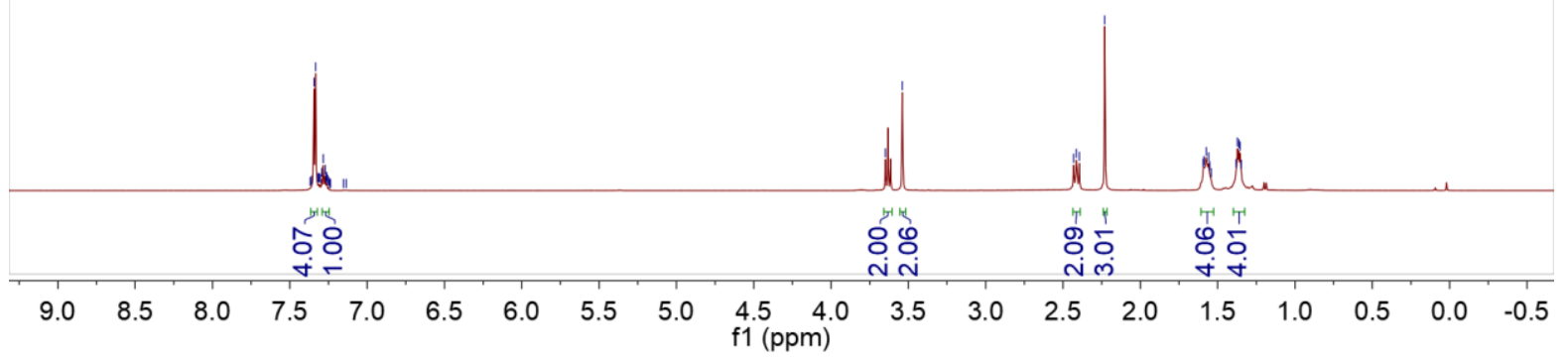




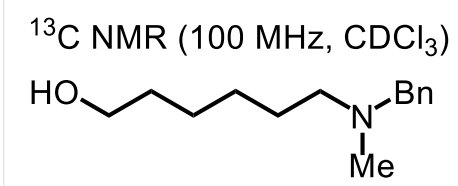

2d

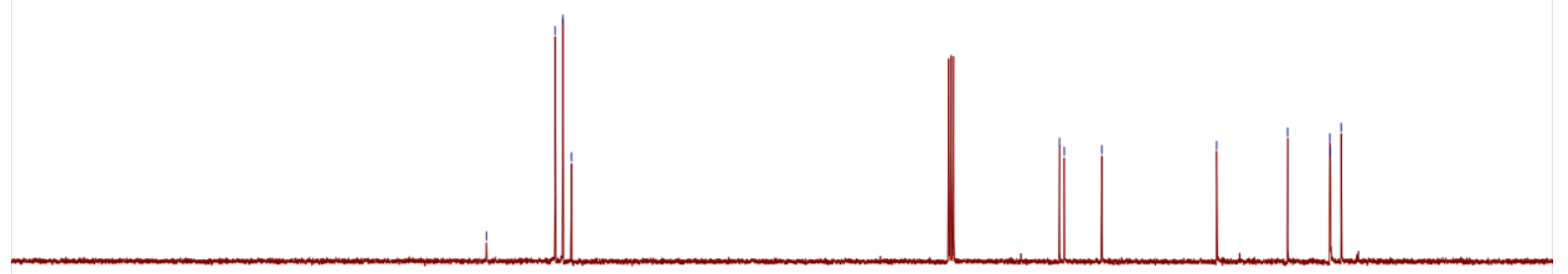

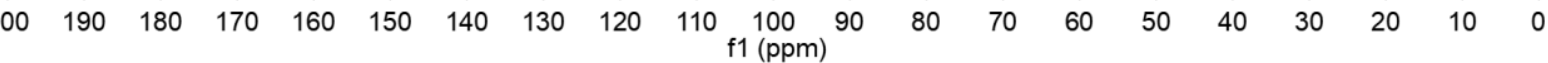

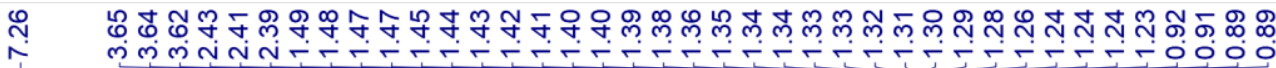

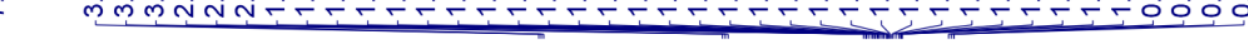

${ }^{1} \mathrm{H}$ NMR $\left(400 \mathrm{MHz}, \mathrm{CDCl}_{3}\right)$<smiles>CCCCCN(CCCCCCO)C(C)(C)CC</smiles>

$2 e$

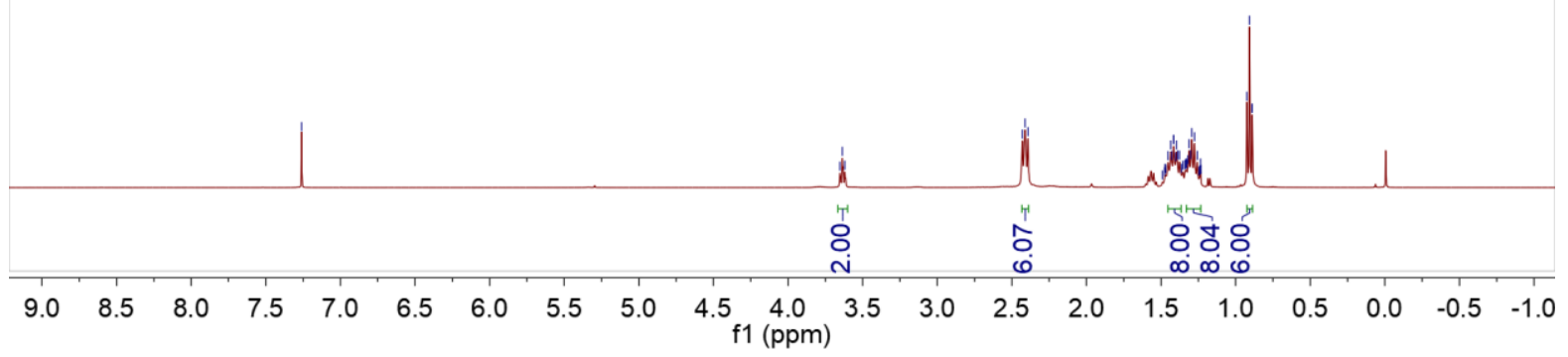


${ }^{13} \mathrm{C} \mathrm{NMR}\left(100 \mathrm{MHz}, \mathrm{CDCl}_{3}\right)$<smiles>CC(C)(C)CCCCCN(CCCCCCO)C(C)(C)C</smiles>

$2 e$

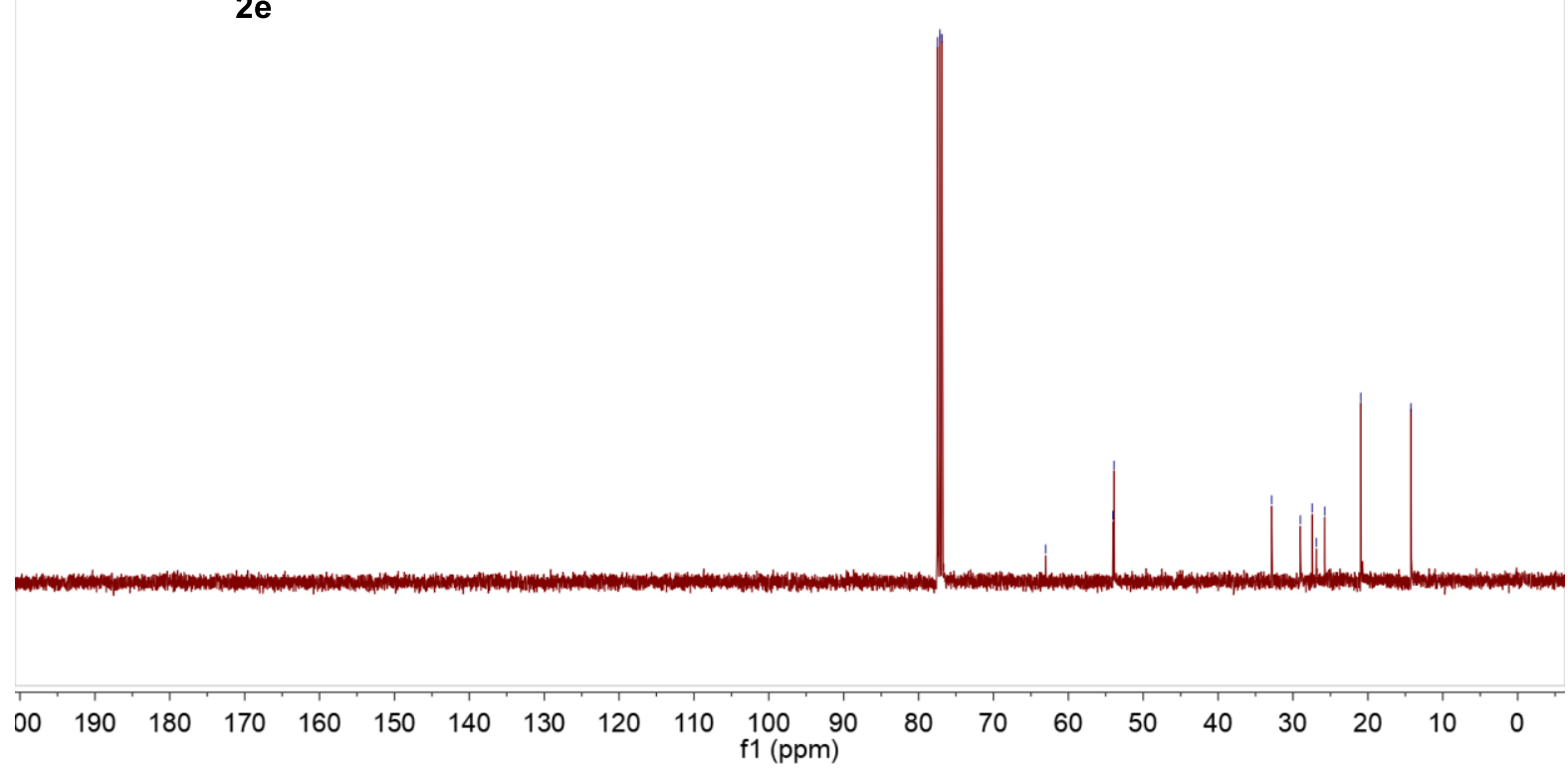

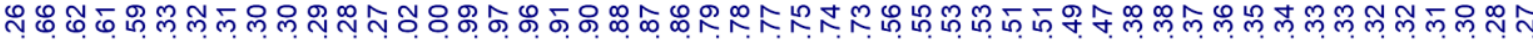

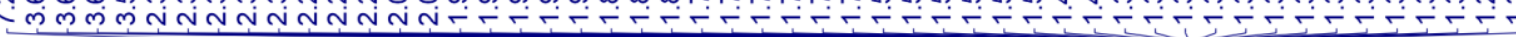

${ }^{1} \mathrm{H}$ NMR $\left(400 \mathrm{MHz}, \mathrm{CDCl}_{3}\right)$<smiles>CC(=O)C1CCN(CCCCCCO)CC1</smiles>

$2 f$

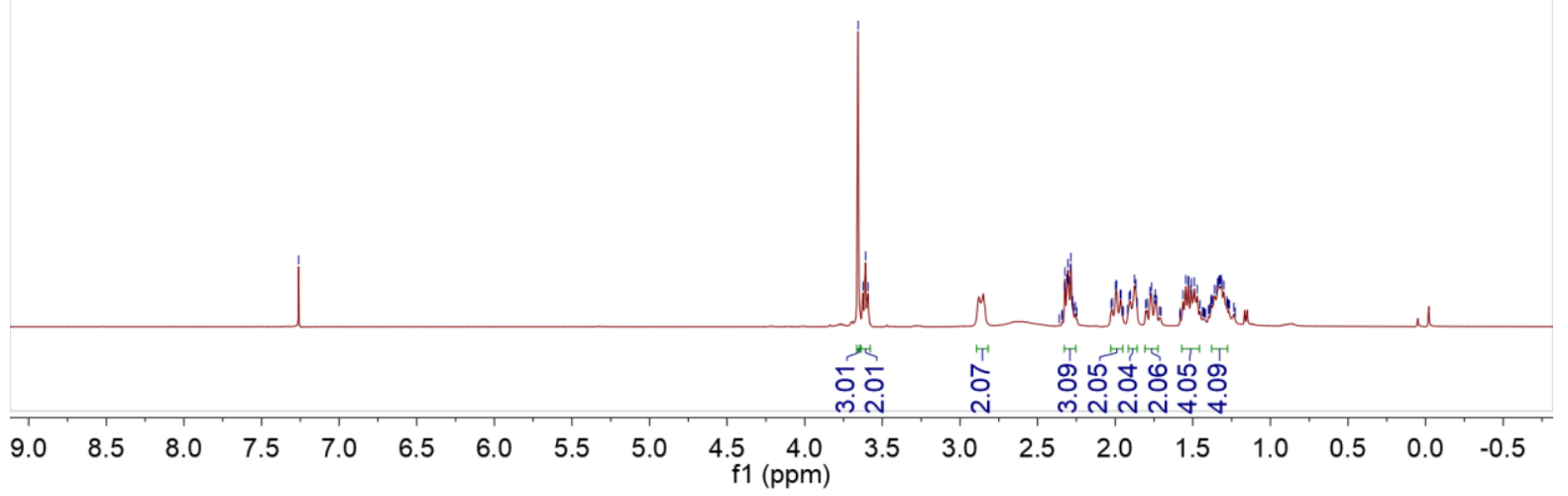


${ }^{13} \mathrm{C}$ NMR $\left(100 \mathrm{MHz}, \mathrm{CDCl}_{3}\right)$

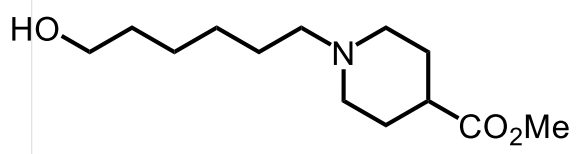

$2 f$

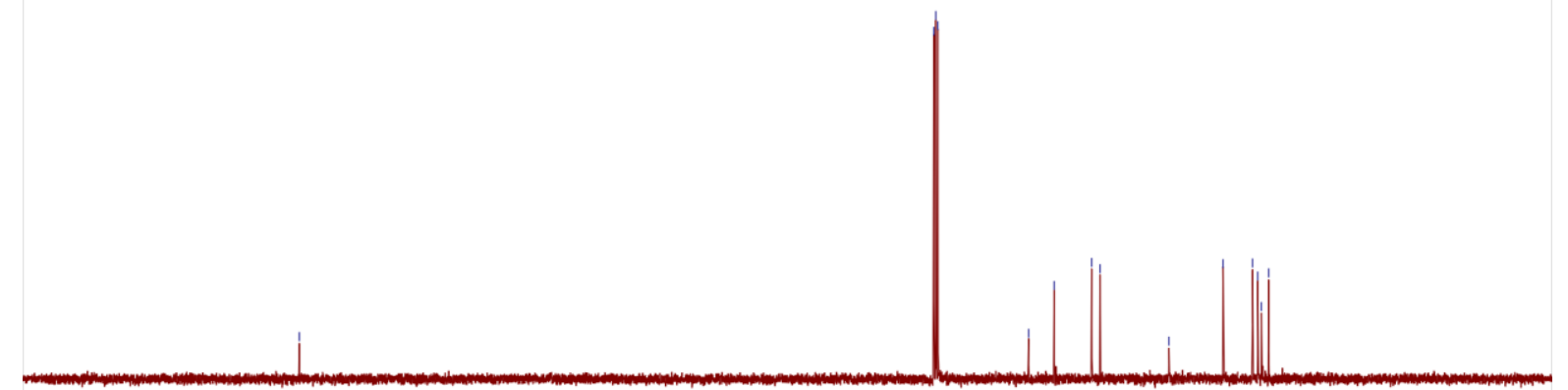

$\begin{array}{lllllllllllllllllllllll}210 & 200 & 190 & 180 & 170 & 160 & 150 & 140 & 130 & 120 & 110 & 100 & 90 & 80 & 70 & 60 & 50 & 40 & 30 & 20 & 10 & 0 & -10\end{array}$

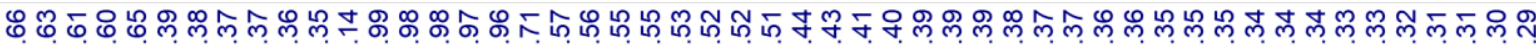

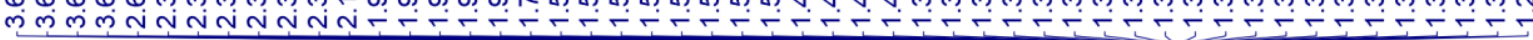

${ }^{1} \mathrm{H} \mathrm{NMR}\left(400 \mathrm{MHz}, \mathrm{CDCl}_{3}\right)$<smiles>CO[C@H]1CCCN(CCCCCCO)C1</smiles>

$2 \mathrm{~g}$

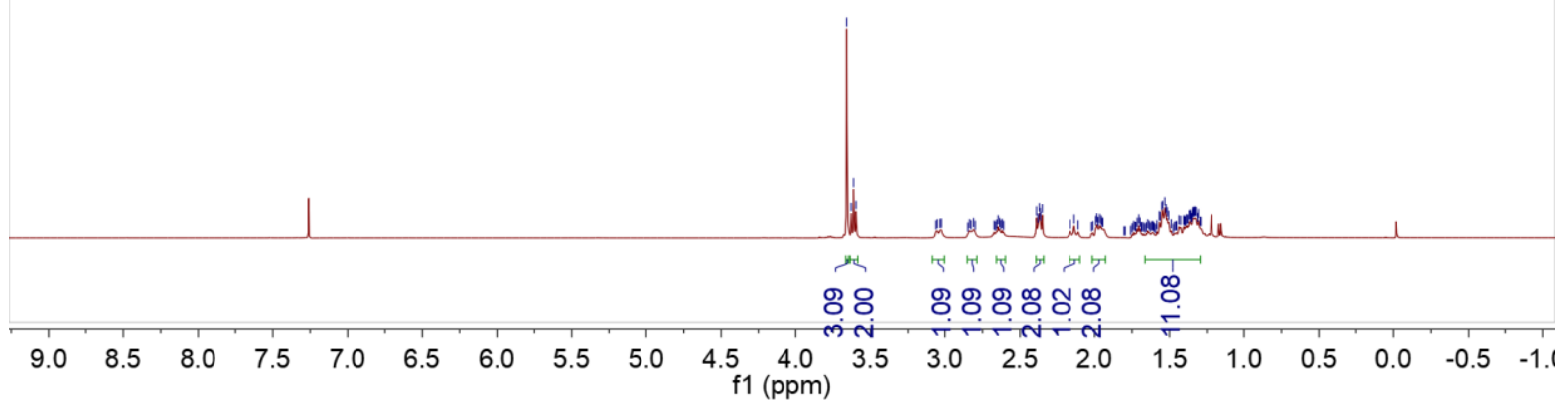



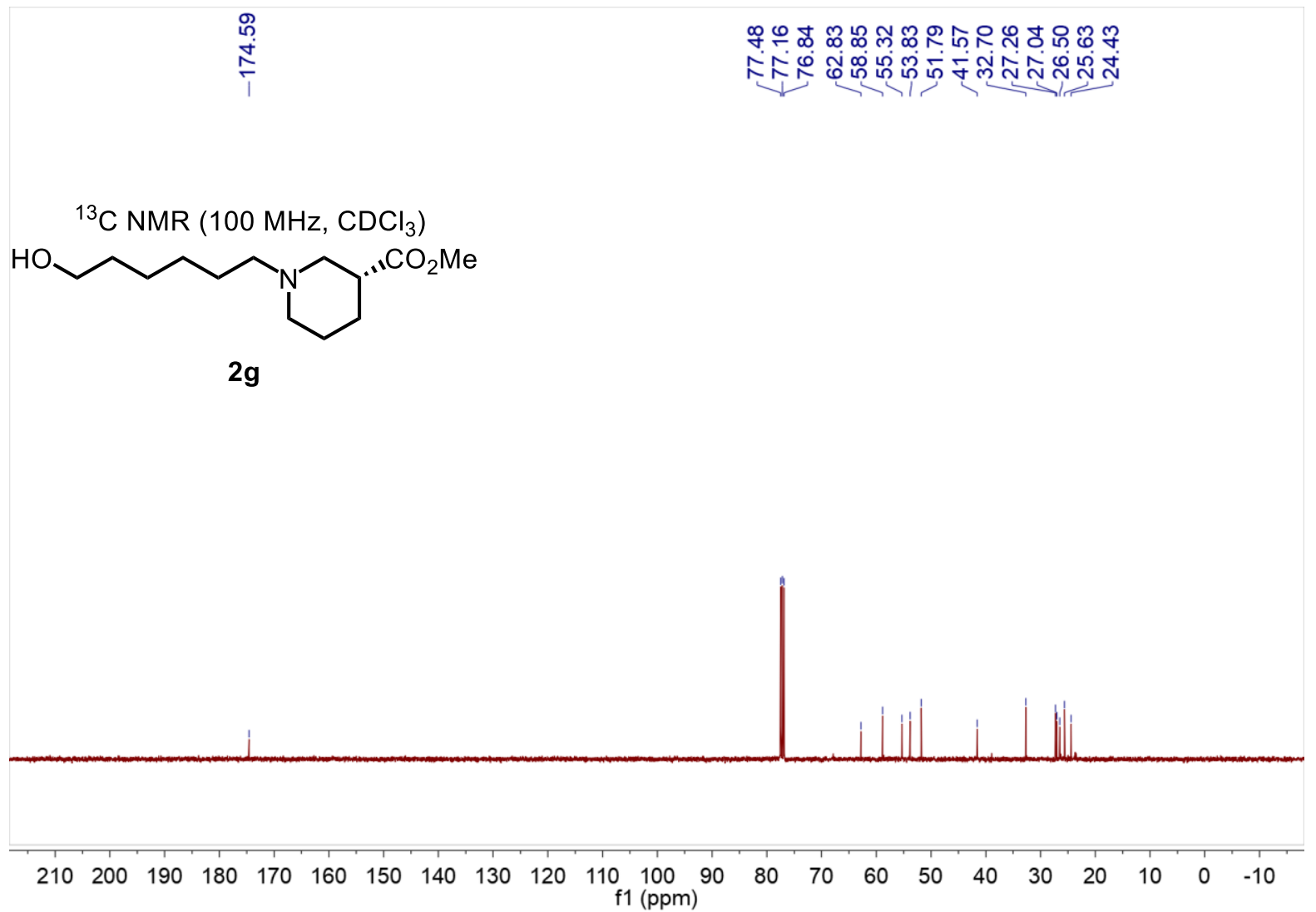

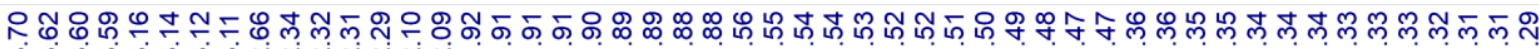

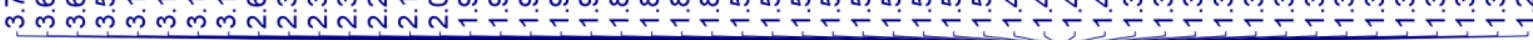<smiles>CO[C@H]1CCCN1CCCCCCO</smiles>

$2 \mathrm{~h}$

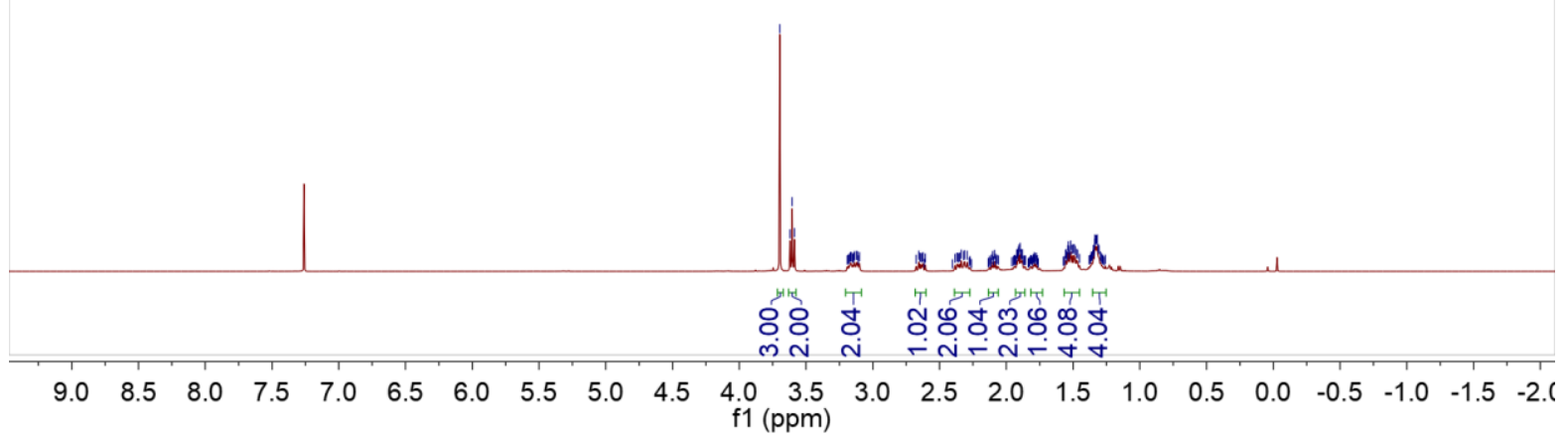




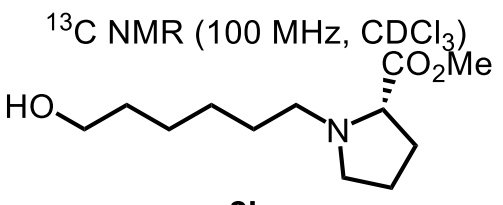

$2 h$

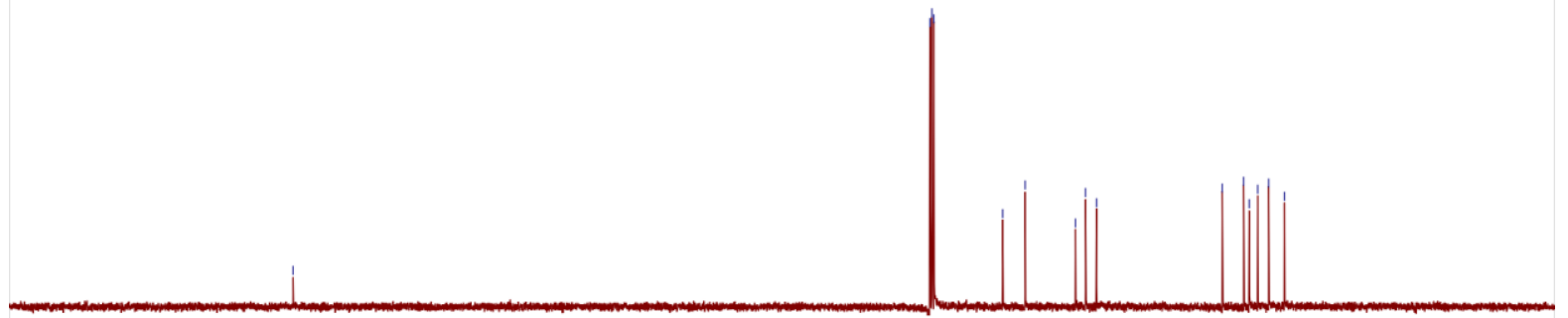

$\begin{array}{lllllllllllllllllllllll}210 & 200 & 190 & 180 & 170 & 160 & 150 & 140 & 130 & 120 & 110 & 100 & 90 & 80 & 70 & 60 & 50 & 40 & 30 & 20 & 10 & 0 & -10\end{array}$

i

${ }^{1} \mathrm{H}$ NMR $\left(400 \mathrm{MHz} \mathrm{CDCl}_{3}\right)$

$\mathrm{HO}$<smiles>CN1CCOCC1</smiles>

$2 \mathrm{i}$

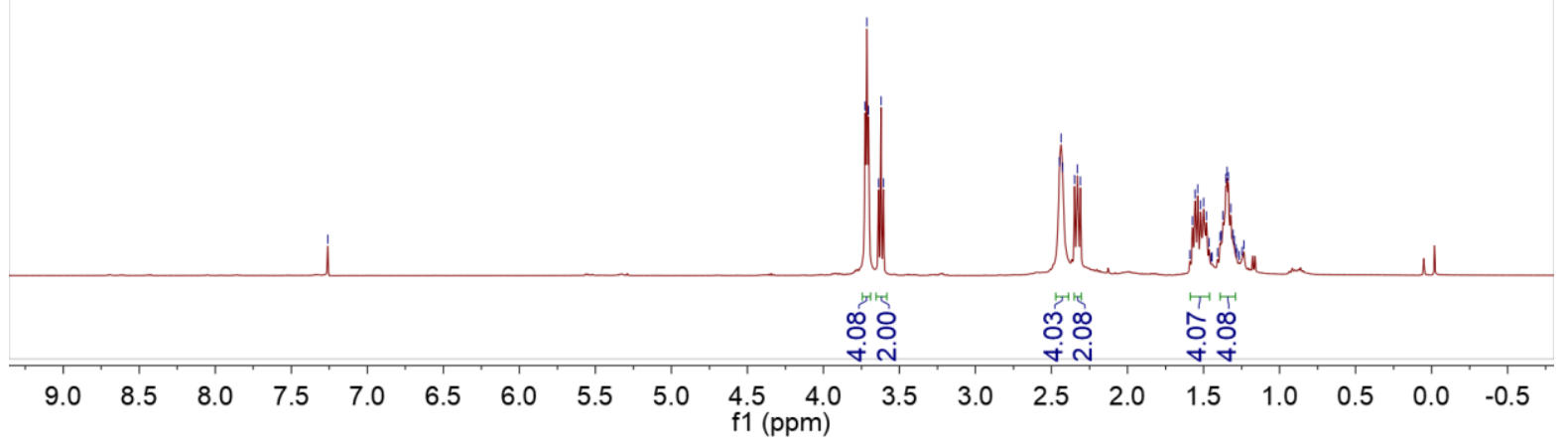




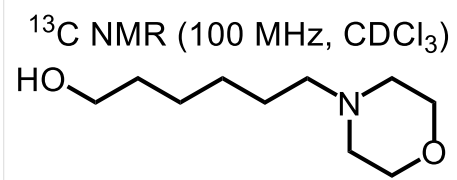

$\mathbf{2 i}$

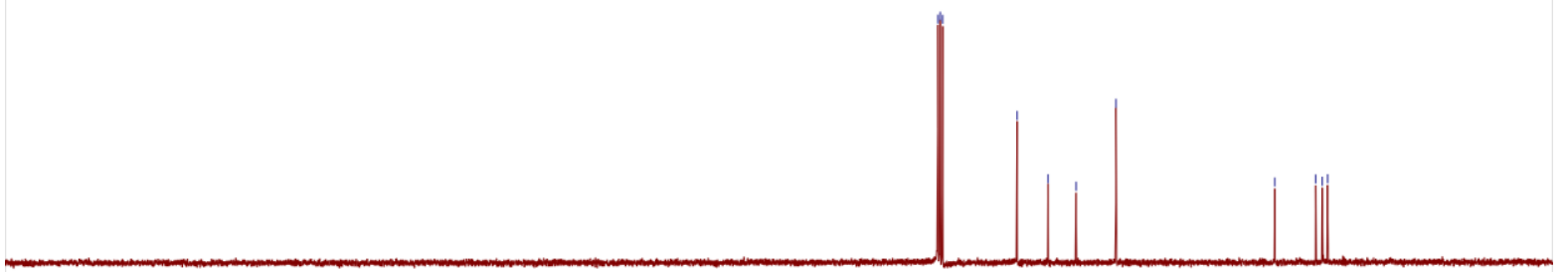

$\begin{array}{lllllllllllllllllllll}00 & 190 & 180 & 170 & 160 & 150 & 140 & 130 & 120 & 110 & \begin{array}{c}100 \\ \mathrm{f} 1(\mathrm{ppm})\end{array} & 90 & 80 & 70 & 60 & 50 & 40 & 30 & 20 & 10 & 0\end{array}$

ஸ̣ ำm m m

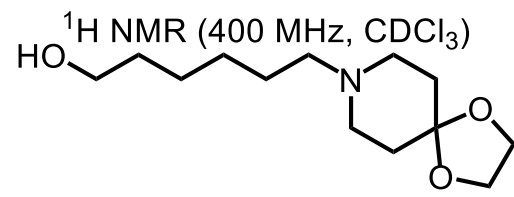

$2 \mathbf{j}$

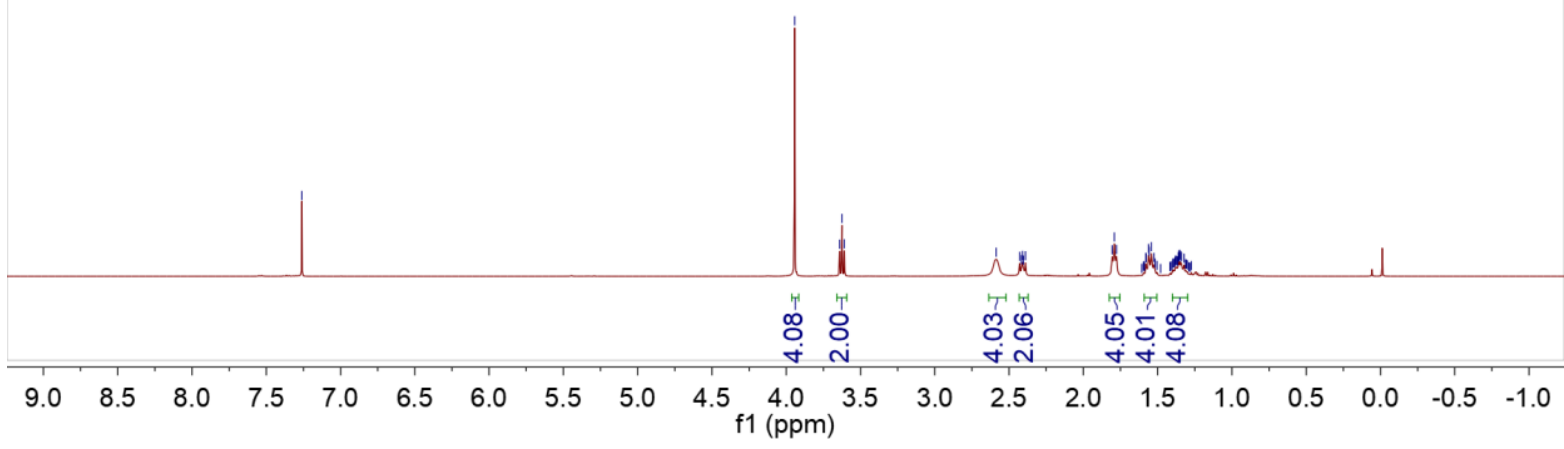




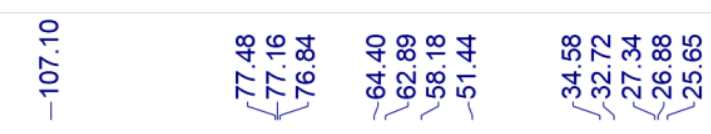

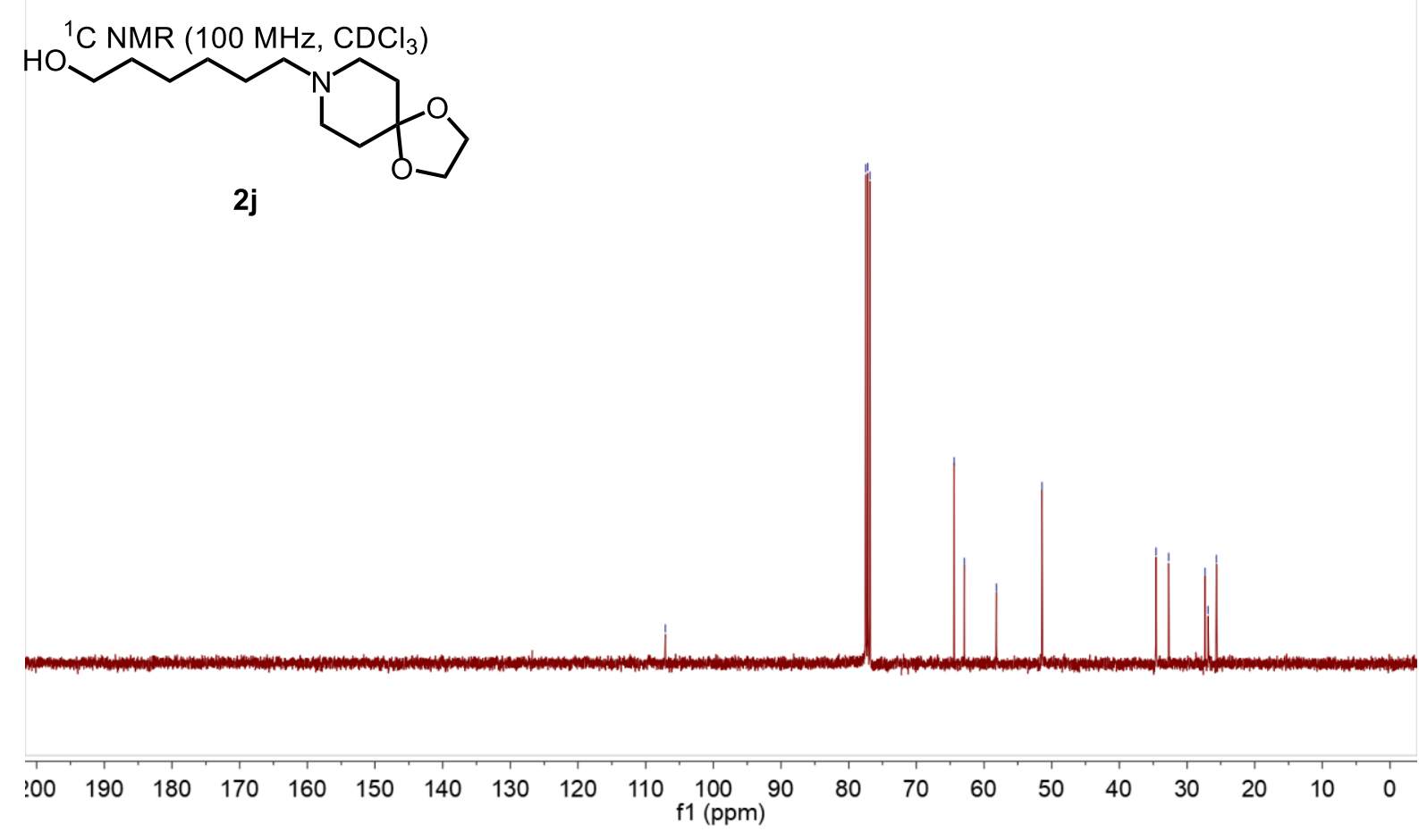

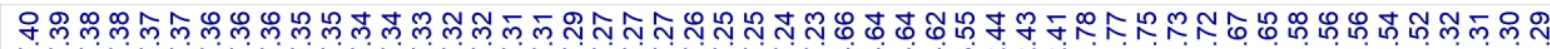

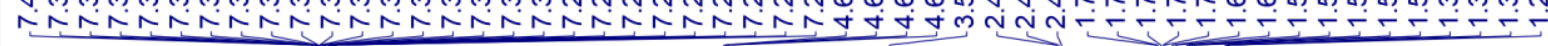

${ }^{1} \mathrm{H}$ NMR $\left(400 \mathrm{MHz}, \mathrm{CDCl}_{3}\right)$<smiles>OC(CCCCN(Cc1ccccc1)Cc1ccccc1)c1ccccc1</smiles>

2k

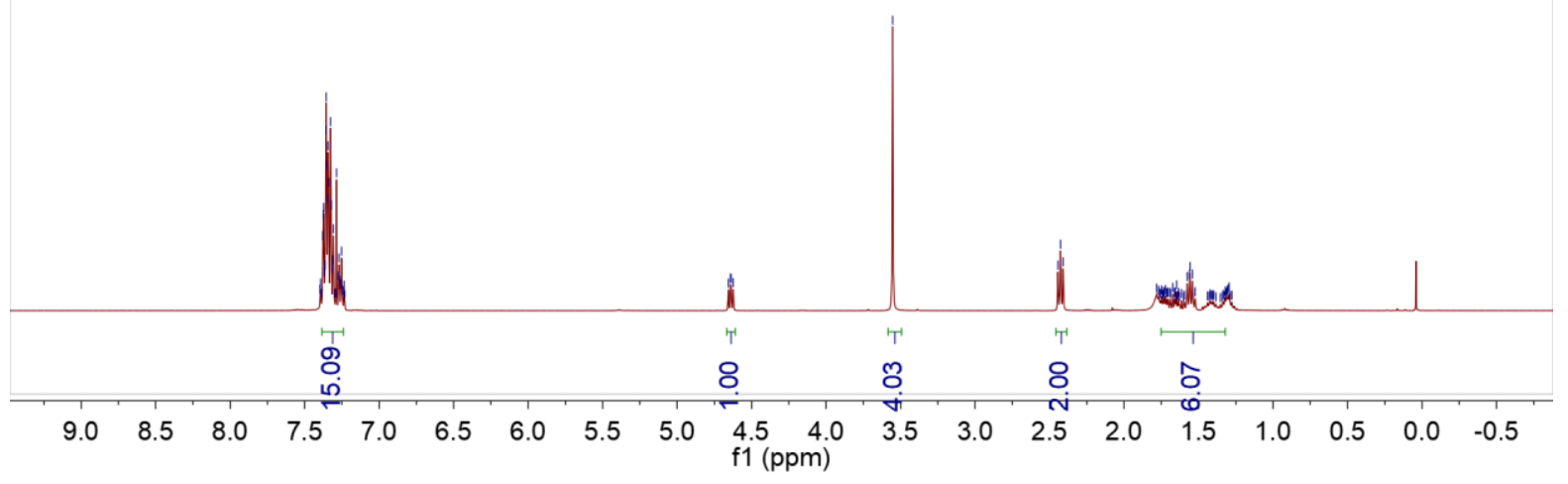




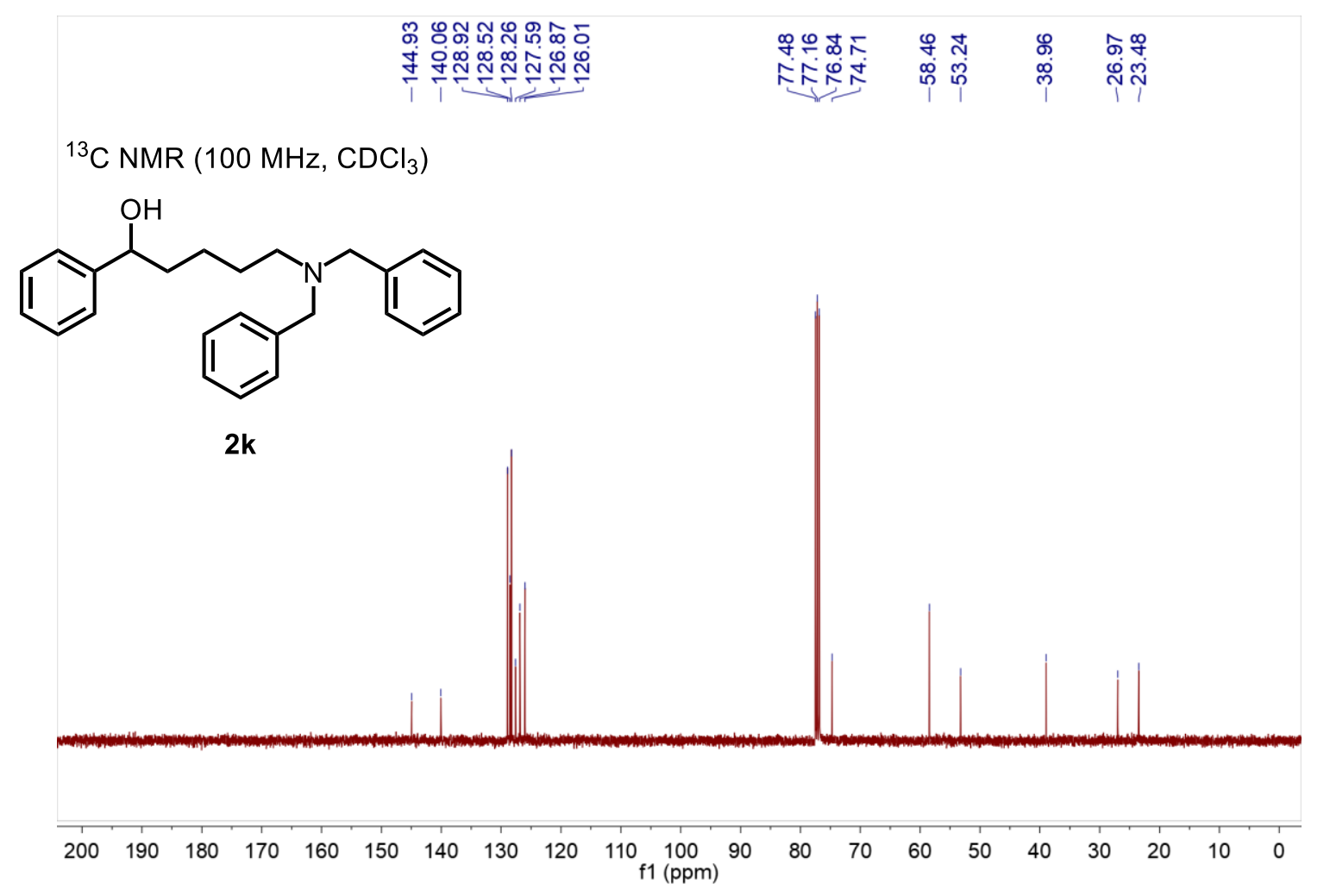

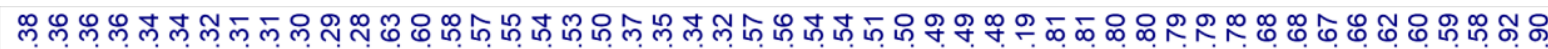

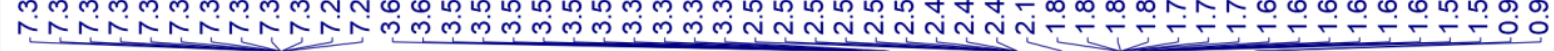

${ }^{1} \mathrm{H}$ NMR $\left(400 \mathrm{MHz}, \mathrm{CDCl}_{3}\right)$

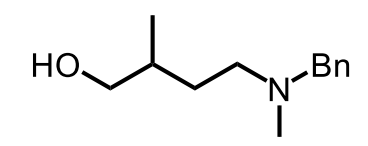

2I

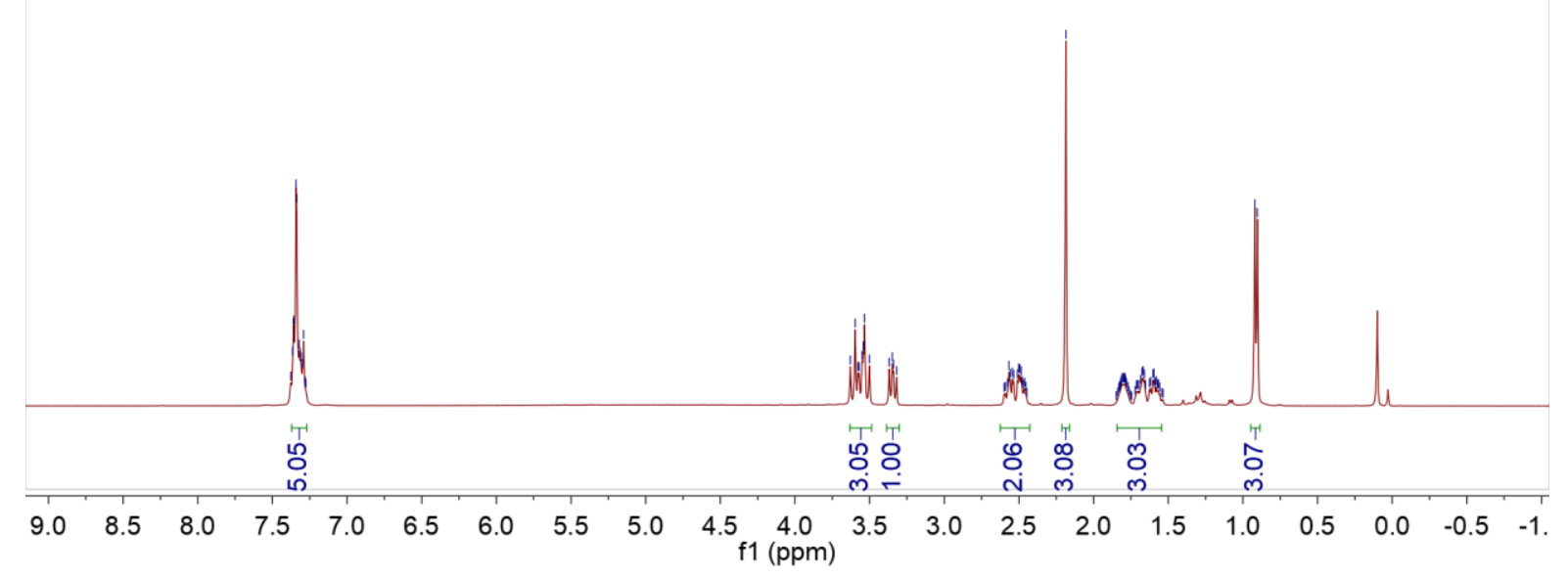


${ }^{13} \mathrm{C}$ NMR (100 MHz, $\mathrm{CDCl}_{3}$ )<smiles>CC(CO)CCN(C)Cc1ccccc1</smiles>

2I

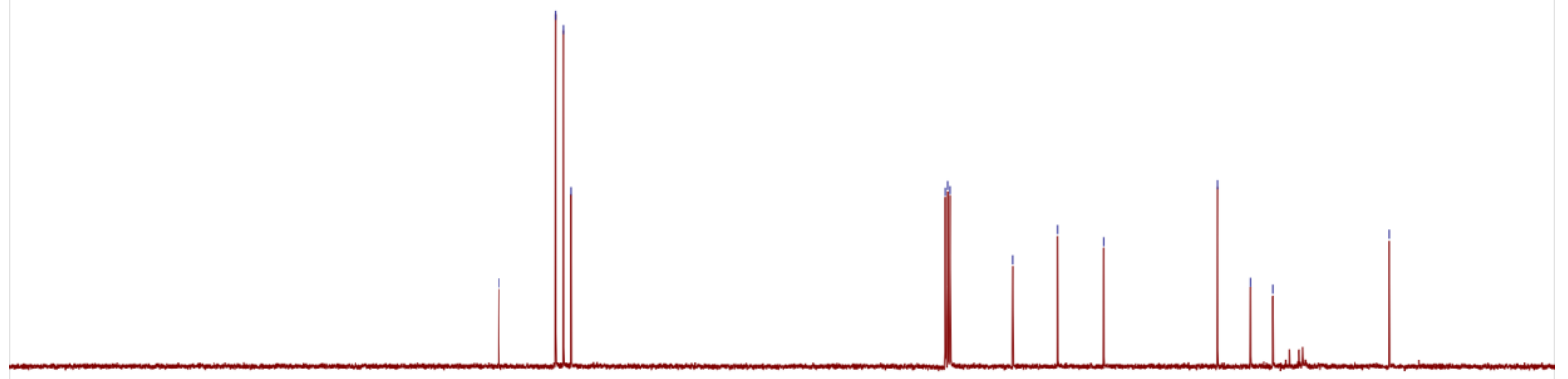

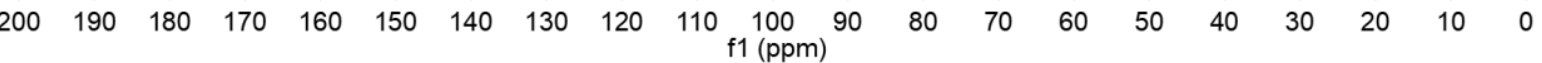

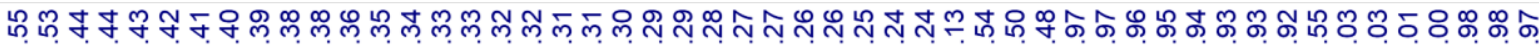

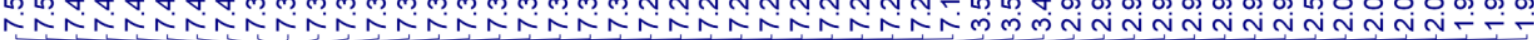

${ }^{1} \mathrm{H}$ NMR $\left(400 \mathrm{MHz}, \mathrm{CDCl}_{3}\right)$<smiles>OCC1CCN(Cc2ccccc2)CC1</smiles>

$2 m$

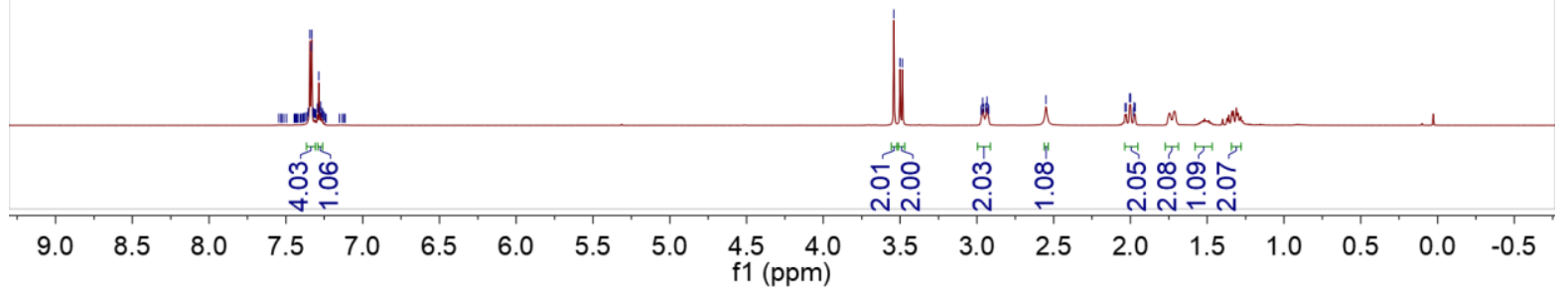




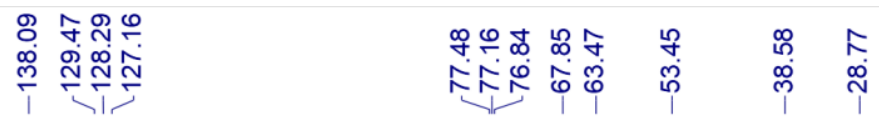

${ }^{13} \mathrm{C}$ NMR $\left(100 \mathrm{MHz}, \mathrm{CDCl}_{3}\right)$<smiles>OCC1CCN(Cc2ccccc2)CC1</smiles>

$2 m$

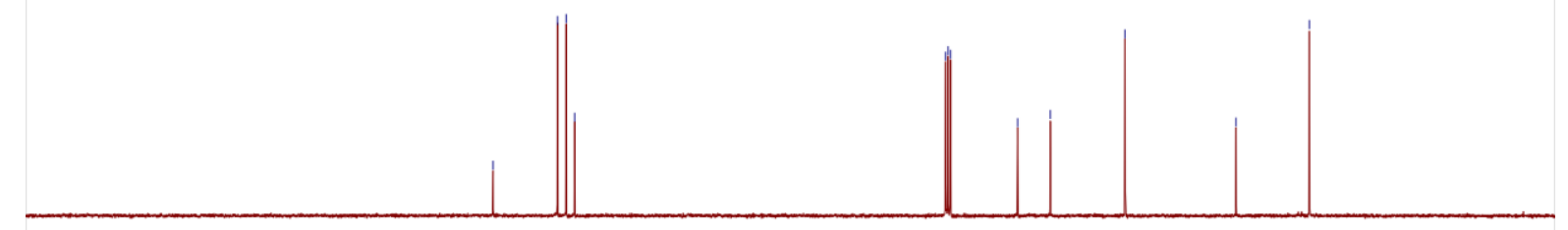

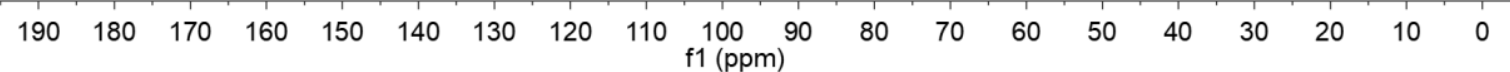

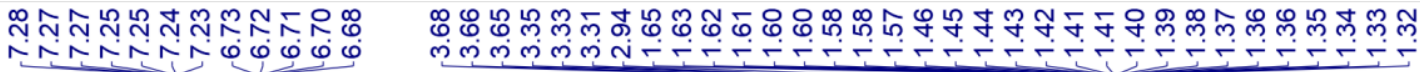

${ }^{1} \mathrm{H} \mathrm{NMR}\left(400 \mathrm{MHz}, \mathrm{CDCl}_{3}\right)$

$\overbrace{\substack{N^{-} \\ M e}}^{\text {POPh }}$

2n

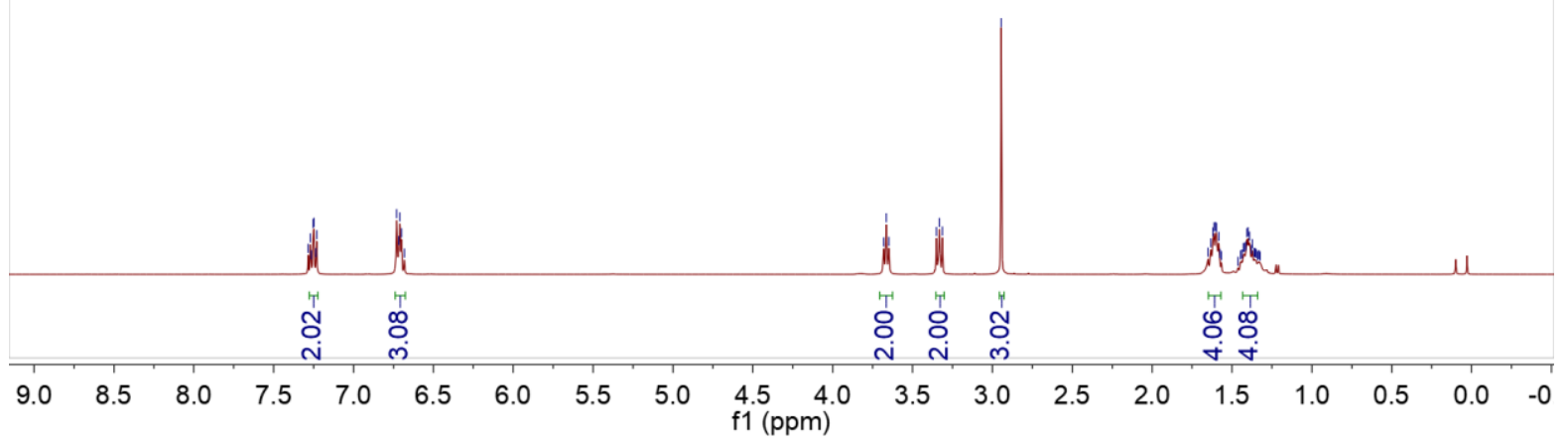




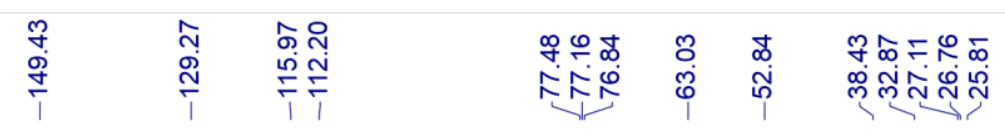

${ }^{13} \mathrm{C} N M R\left(100 \mathrm{MHz}, \mathrm{CDCl}_{3}\right)$

2n

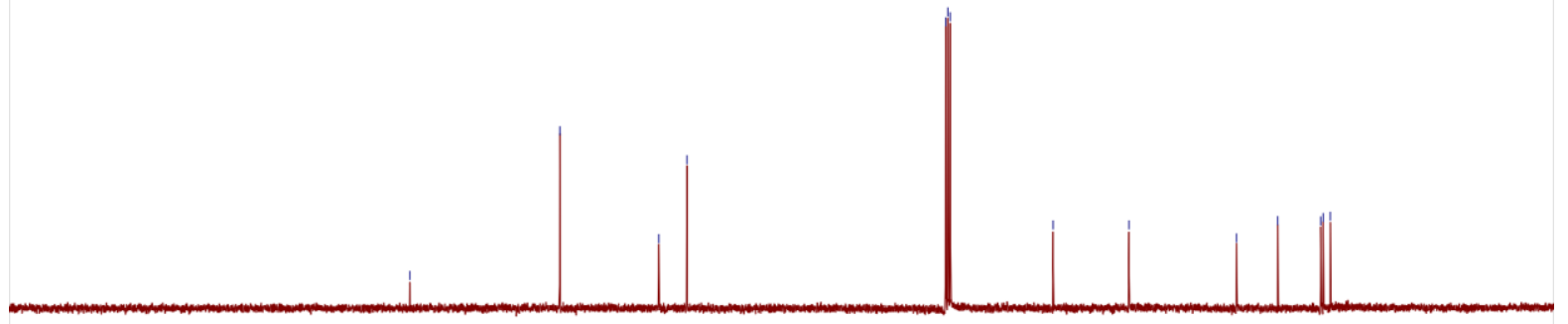

$\begin{array}{lllllllllllllllllllll}200 & 190 & 180 & 170 & 160 & 150 & 140 & 130 & 120 & 110 \underset{\mathrm{f} 1(\mathrm{ppm})}{100} \mathbf{9 0} & 80 & 70 & 60 & 50 & 40 & 30 & 20 & 10 & 0\end{array}$

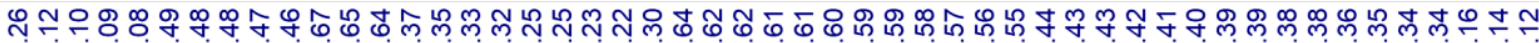

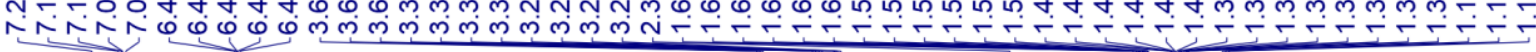

${ }^{1} \mathrm{H}$ NMR $\left(400 \mathrm{MHz}, \mathrm{CDCl}_{3}\right)$<smiles>CCN(CCCCCCO)c1cccc(C)c1</smiles>

20

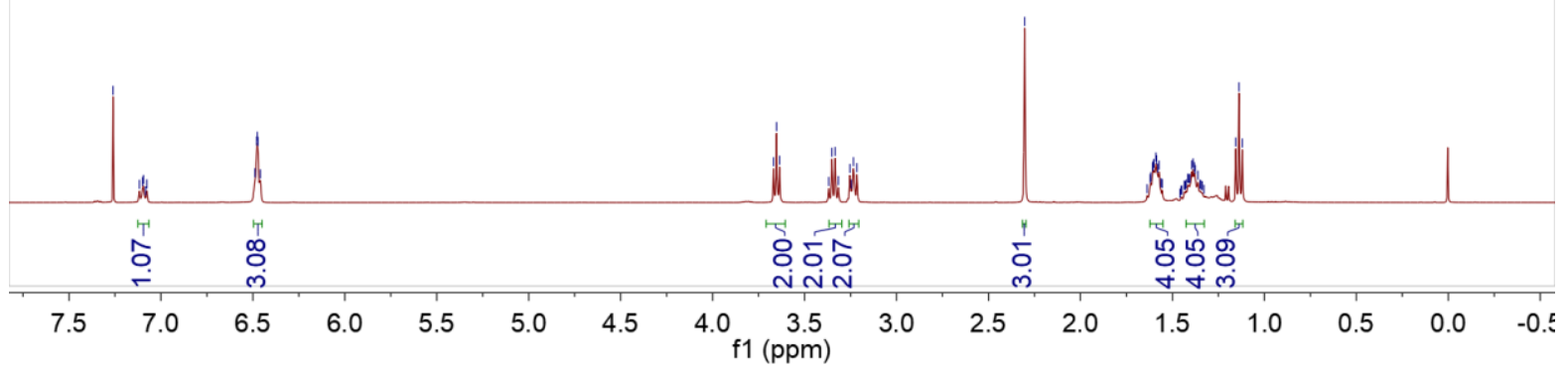




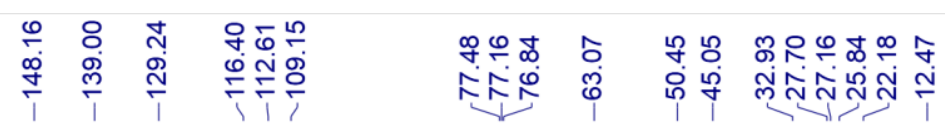

${ }^{13} \mathrm{C} \mathrm{NMR}\left(100 \mathrm{MHz}, \mathrm{CDCl}_{3}\right)$<smiles>CCN(CCCCCCO)c1cccc(C)c1</smiles>

20

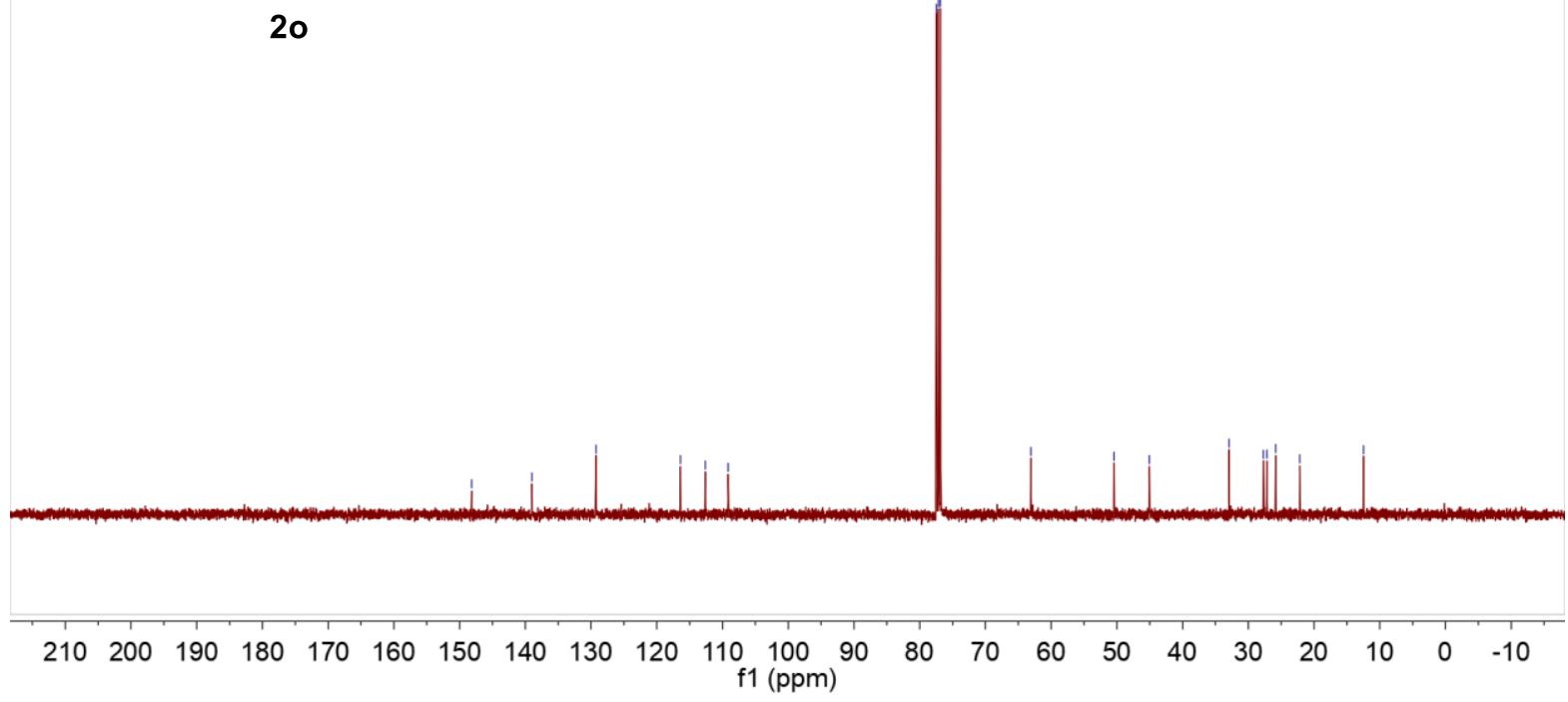

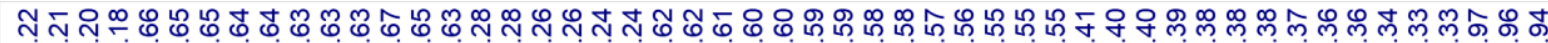

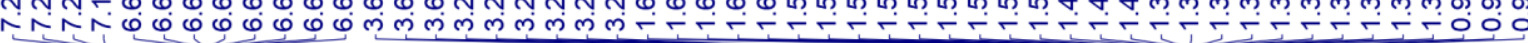<smiles>CC(C)(C)ON(CCCCN)CCCCCCO</smiles>

$2 p$

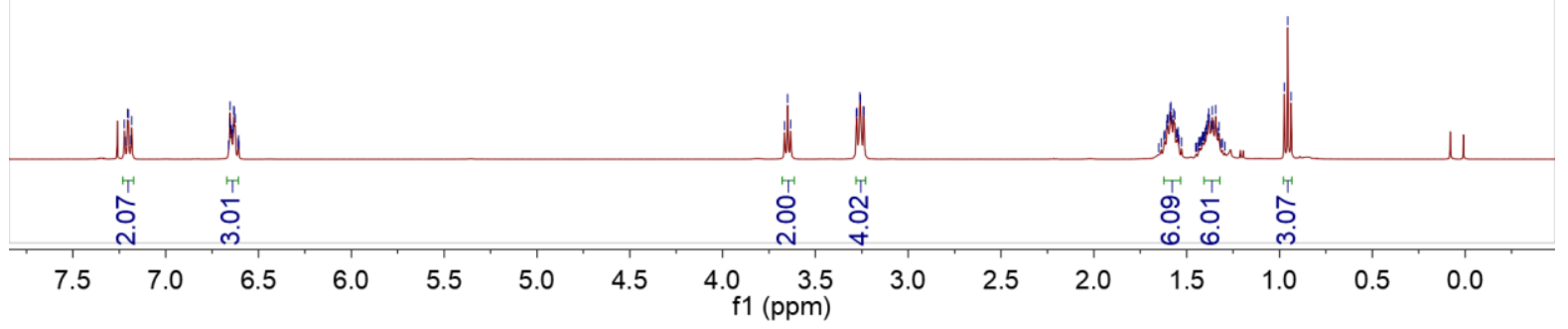




\begin{tabular}{|c|c|c|c|c|c|}
\hline 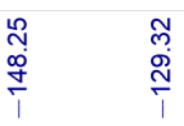 & 鱼 & 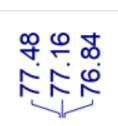 & $\begin{array}{l}\text { पे } \\
0 \\
0 \\
0\end{array}$ & 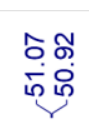 & 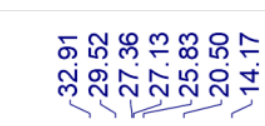 \\
\hline
\end{tabular}

${ }^{13} \mathrm{C}$ NMR $\left(100 \mathrm{MHz}, \mathrm{CDCl}_{3}\right)$

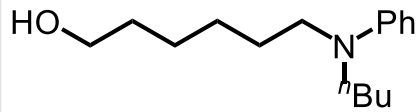

$2 p$

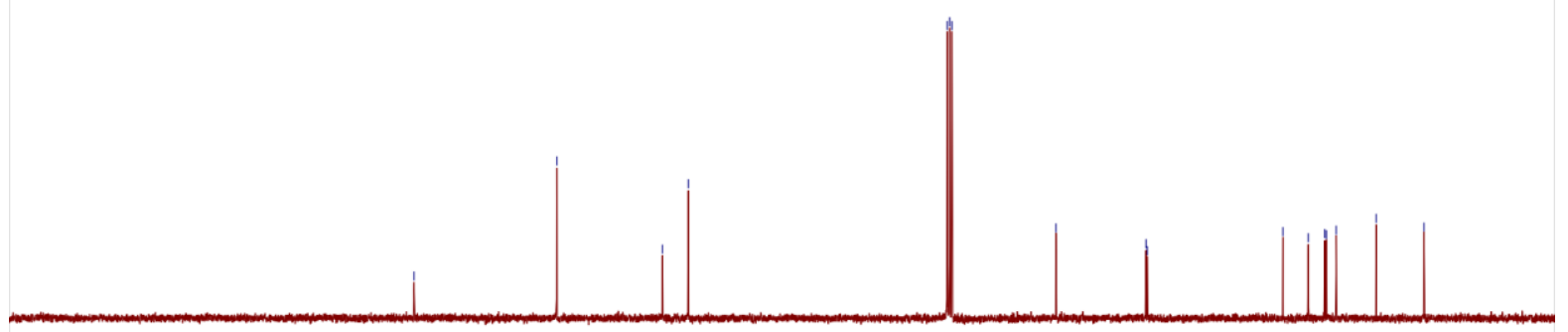

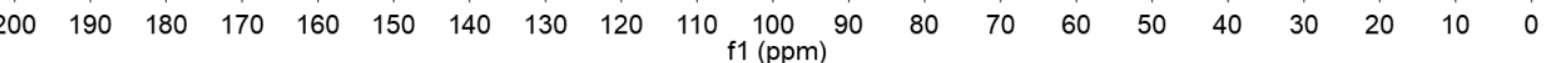

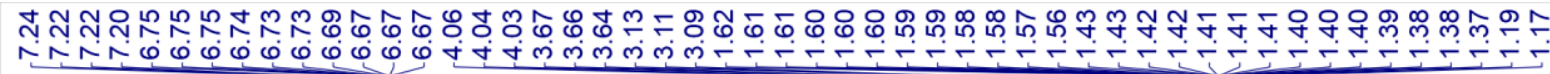

${ }^{1} \mathrm{H}$ NMR $\left(400 \mathrm{MHz}, \mathrm{CDCl}_{3}\right)$

$\mathrm{HO}$<smiles>CCCCCCN(CC)Pc1ccccc1</smiles>

$2 q$

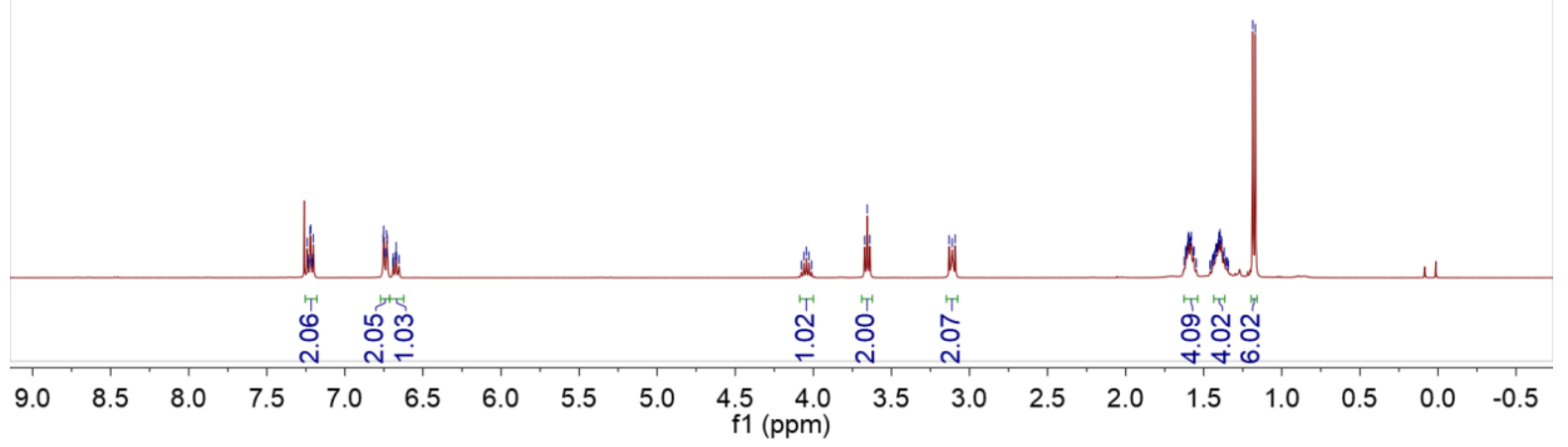




\begin{tabular}{|c|c|c|c|c|c|}
\hline \begin{tabular}{l} 
S. \\
o \\
\multirow{+}{+}{}
\end{tabular} & $\begin{array}{l}\stackrel{0}{1} \\
\stackrel{N}{N}\end{array}$ & 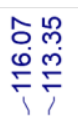 & 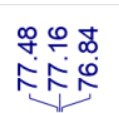 & 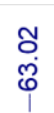 & 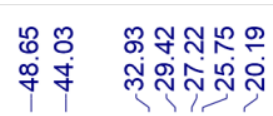 \\
\hline
\end{tabular}

${ }^{13} \mathrm{C} \mathrm{NMR}\left(100 \mathrm{MHz}, \mathrm{CDCl}_{3}\right)$

$\mathrm{HO}$<smiles>CCCCCCCN(P)Pc1ccccc1</smiles>

2q

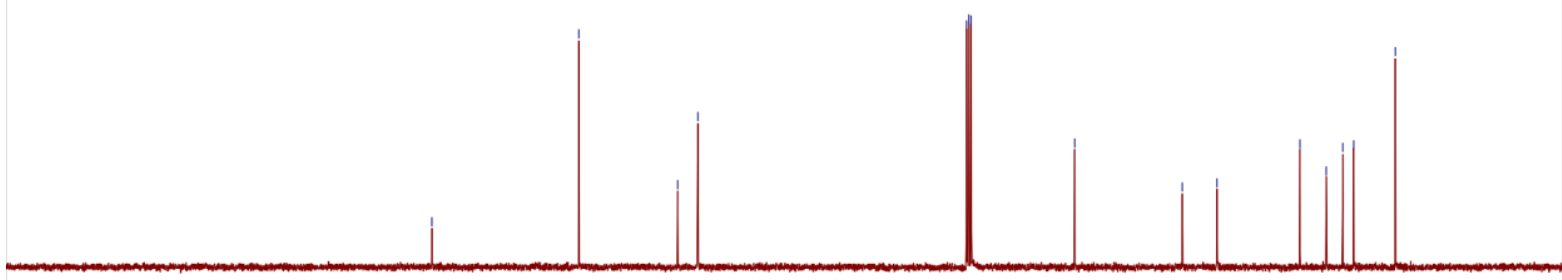

$\begin{array}{lllllllllllllllllllll}200 & 190 & 180 & 170 & 160 & 150 & 140 & 130 & 120 & \begin{array}{c}110 \\ \mathrm{f} 1(\mathrm{ppm})\end{array} & 90 & 80 & 70 & 60 & 50 & 40 & 30 & 20 & 10 & 0\end{array}$

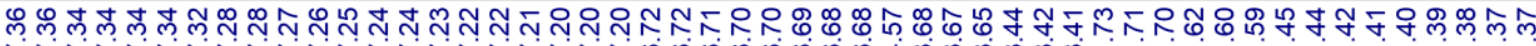

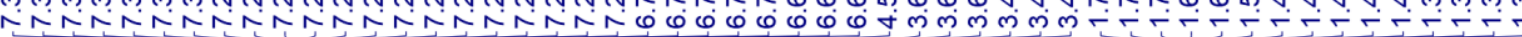

${ }^{1} \mathrm{H} \mathrm{NMR}\left(400 \mathrm{MHz}, \mathrm{CDCl}_{3}\right)$<smiles>OCCCCCCN(Cc1ccccc1)c1ccccc1</smiles>

$2 \mathbf{r}$

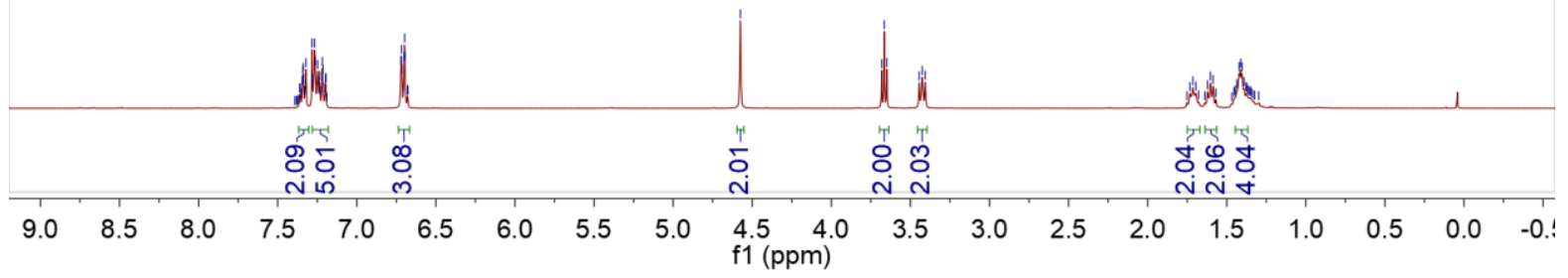


${ }^{13} \mathrm{C} N M R\left(100 \mathrm{MHz}, \mathrm{CDCl}_{3}\right)$

$2 r$

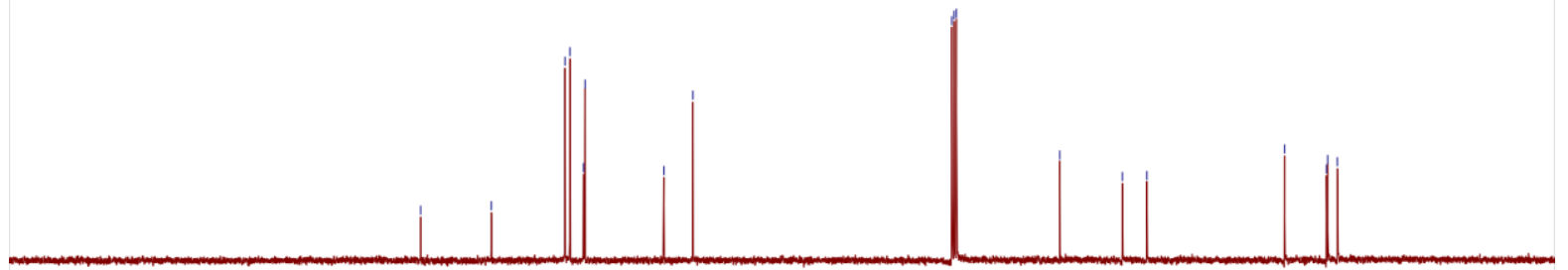

$\begin{array}{lllllllllllllllllllll}200 & 190 & 180 & 170 & 160 & 150 & 140 & 130 & 120 & 110 \underset{\mathrm{f} 1(\mathrm{ppm})}{100} & 90 & 80 & 70 & 60 & 50 & 40 & 30 & 20 & 10 & 0\end{array}$

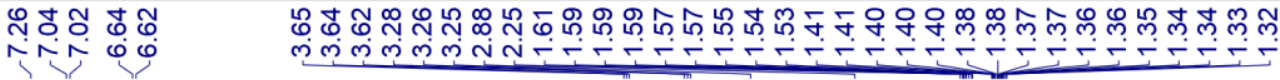

${ }^{1} \mathrm{H}$ NMR $\left(400 \mathrm{MHz}, \mathrm{CDCl}_{3}\right)$<smiles>Cc1ccc(N(C)CCCCCCO)cc1</smiles>

2s

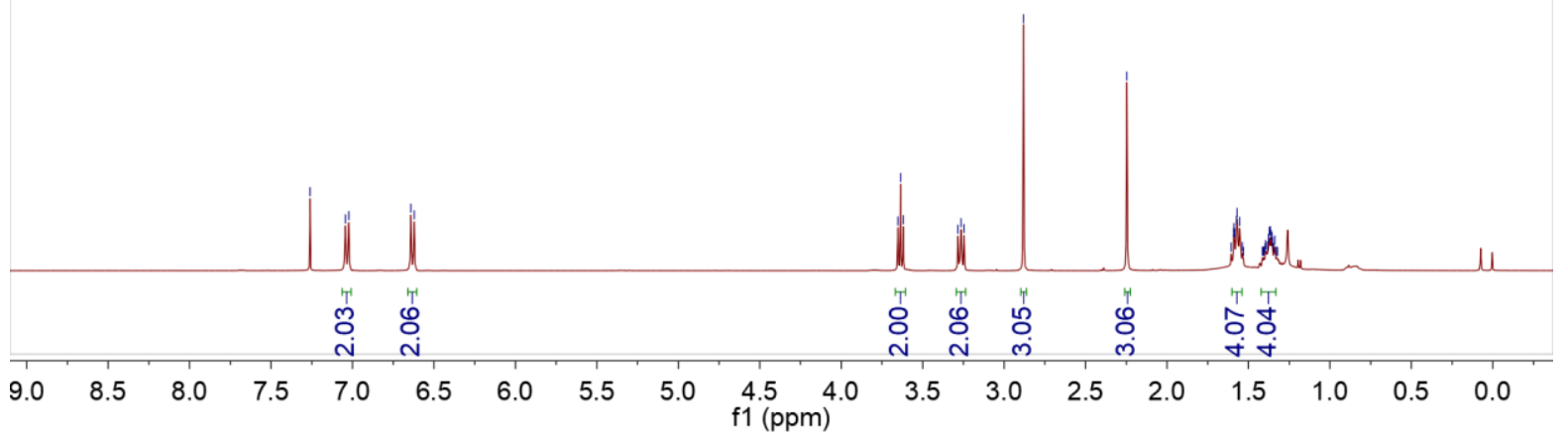




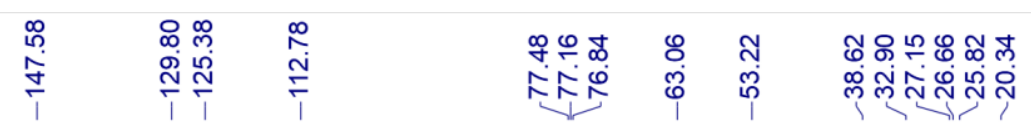<smiles>Cc1ccc(N(C)CCCCCCO)cc1</smiles>

2s

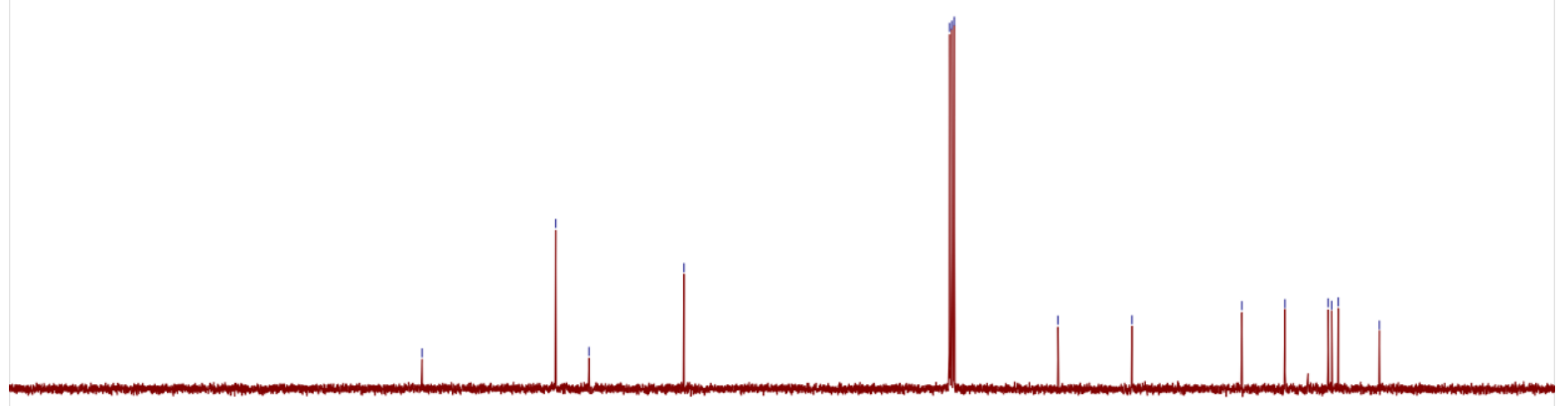

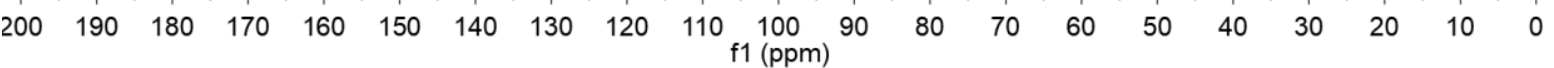

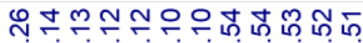

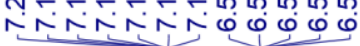

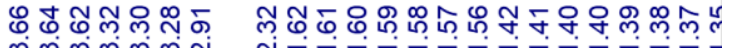
घंलुलिखल

${ }^{1} \mathrm{H}$ NMR $\left(400 \mathrm{MHz}, \mathrm{CDCl}_{3}\right)$<smiles>Cc1cccc(N(C)CCCCCCO)c1</smiles>

$2 t$

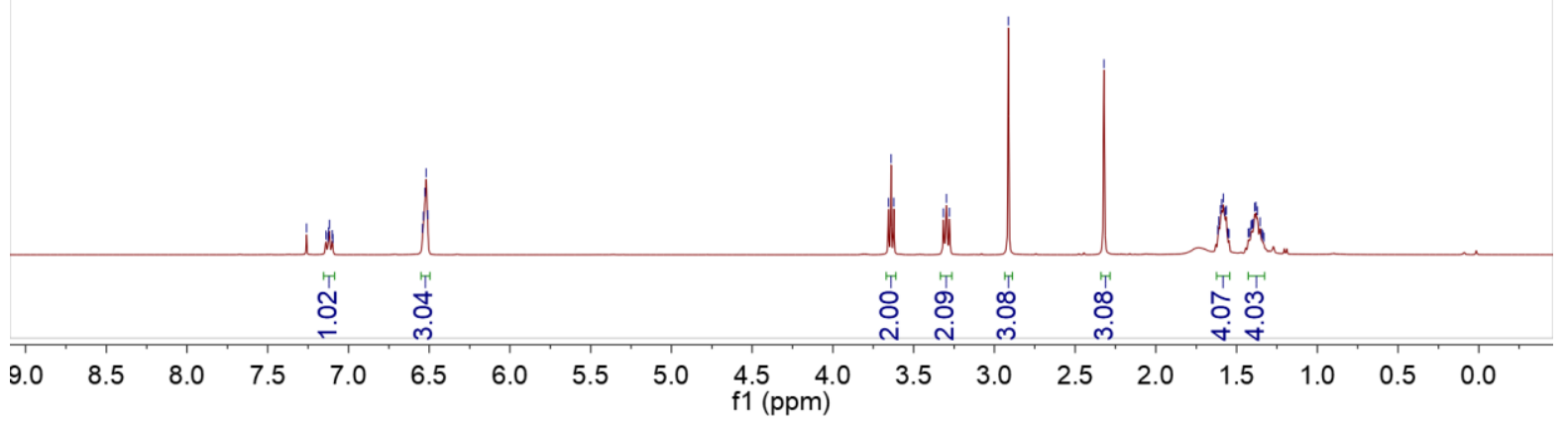




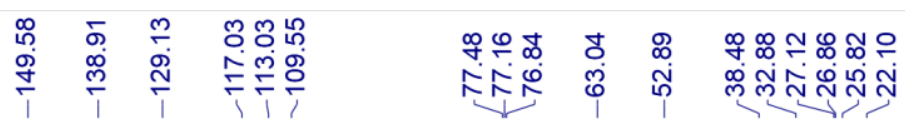

${ }^{13} \mathrm{C}$ NMR $\left(100 \mathrm{MHz}, \mathrm{CDCl}_{3}\right)$<smiles>Cc1cccc(N(C)CCCCCCO)c1</smiles>

$2 t$

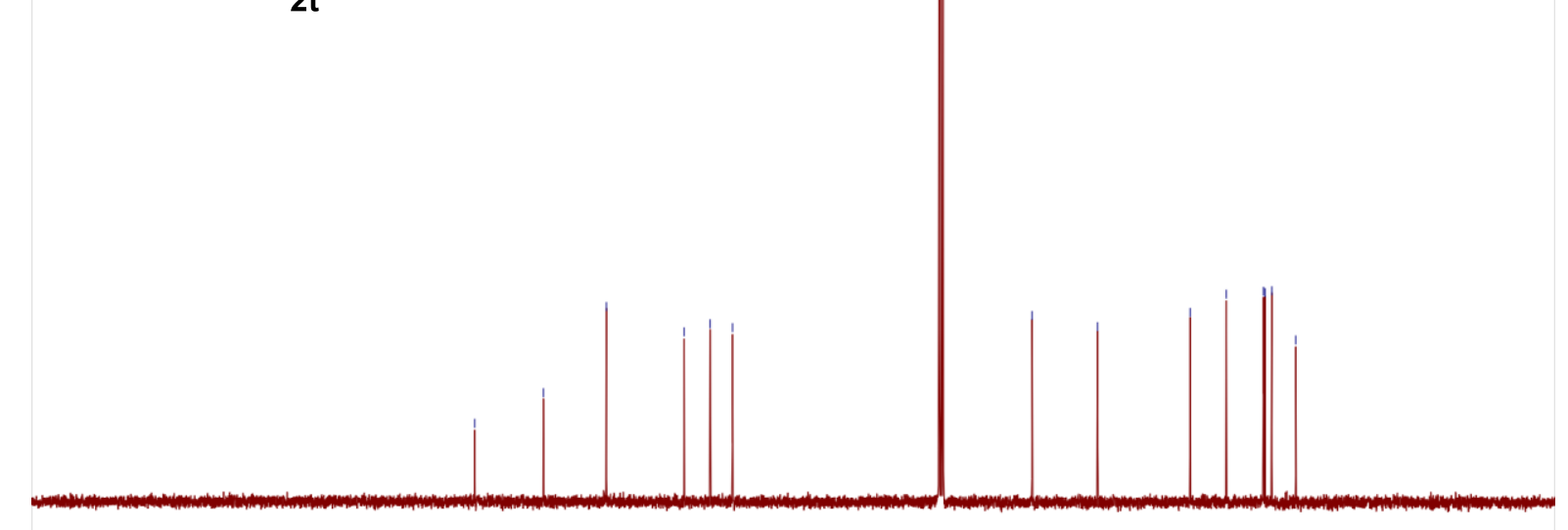

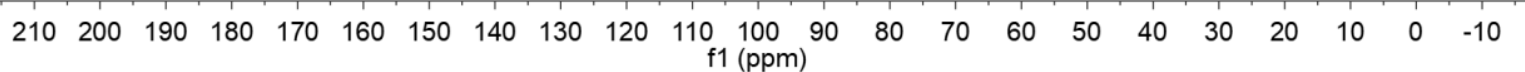

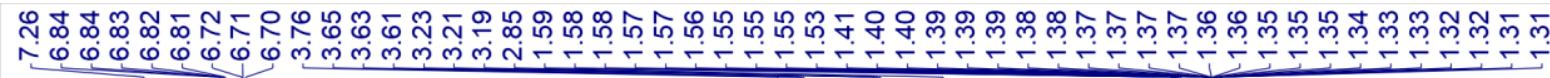

${ }^{1} \mathrm{H}$ NMR $\left(400 \mathrm{MHz}, \mathrm{CDCl}_{3}\right)$<smiles>COc1ccc(N(C)CCCCCCO)cc1</smiles>

$2 u$

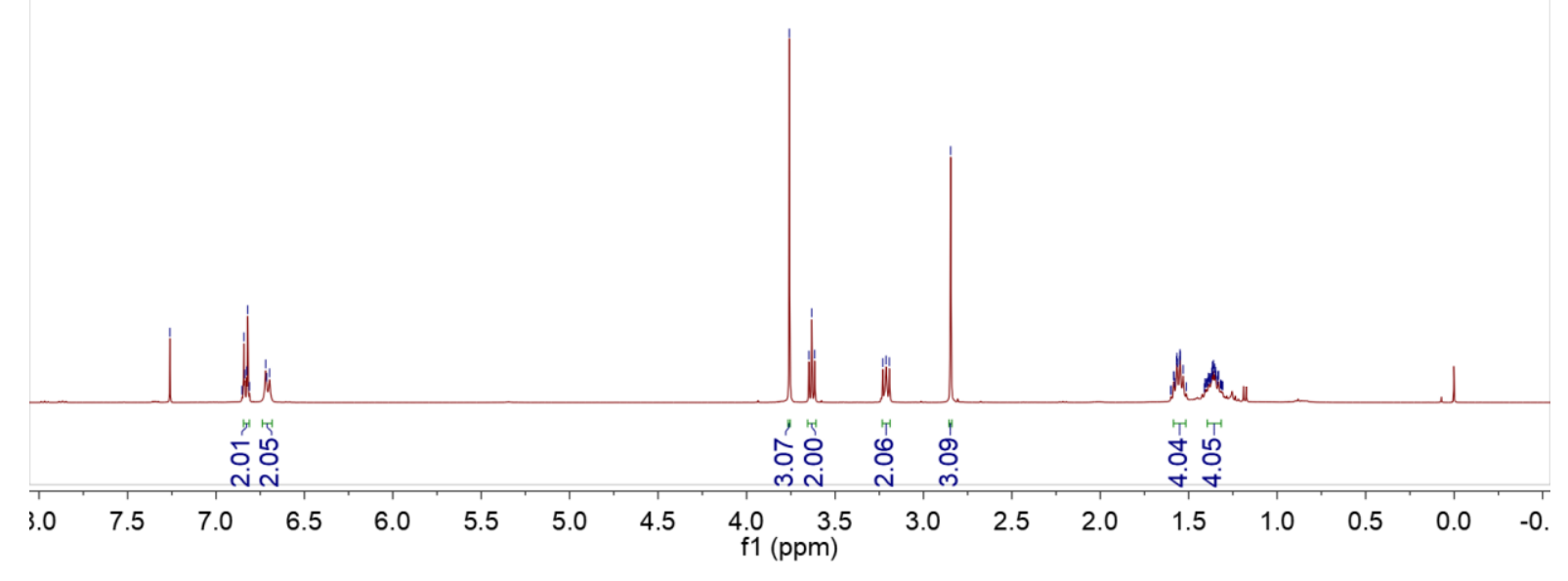




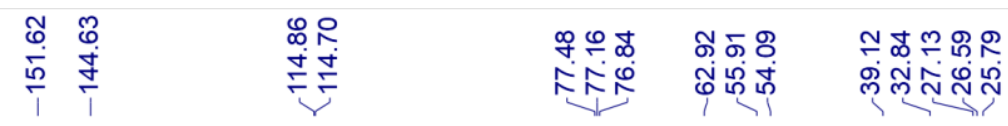

${ }^{13} \mathrm{C} \mathrm{NMR}\left(100 \mathrm{MHz}, \mathrm{CDCl}_{3}\right)$<smiles>COc1ccc(N(C)CCCCCCO)cc1</smiles>

2u

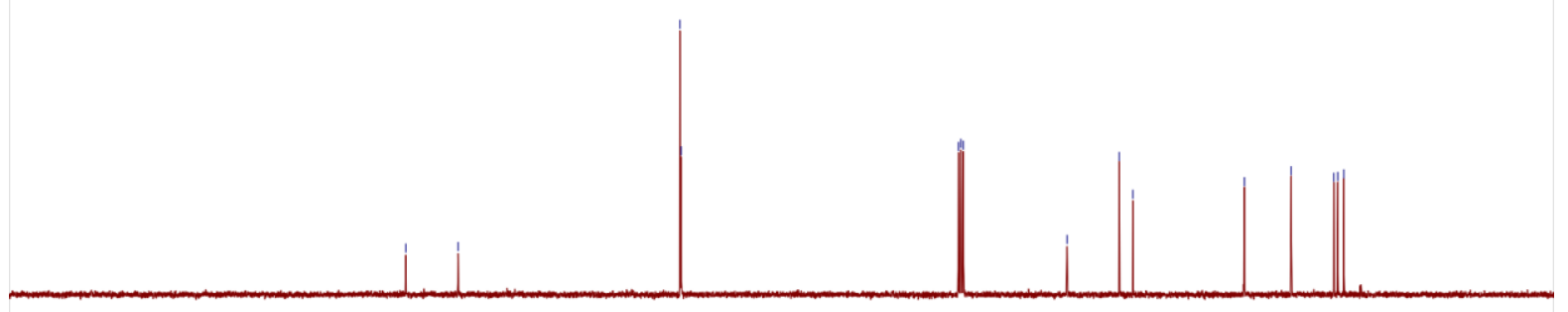

$\begin{array}{llllllllllllllllllll}200 & 190 & 180 & 170 & 160 & 150 & 140 & 130 & 120 & \begin{array}{c}110 \\ \mathrm{f} 1(\mathrm{ppm})\end{array} & \begin{array}{l}100 \\ (\mathrm{ppm}\end{array} & 80 & 70 & 60 & 50 & 40 & 30 & 20 & 10 & 0\end{array}$

๑ุ

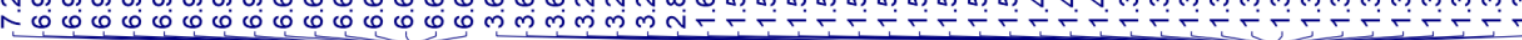

${ }^{1} \mathrm{H} \mathrm{NMR}\left(400 \mathrm{MHz}, \mathrm{CDCl}_{3}\right)$<smiles>CN(CCCCCCO)c1ccc(F)cc1</smiles>

$2 v$

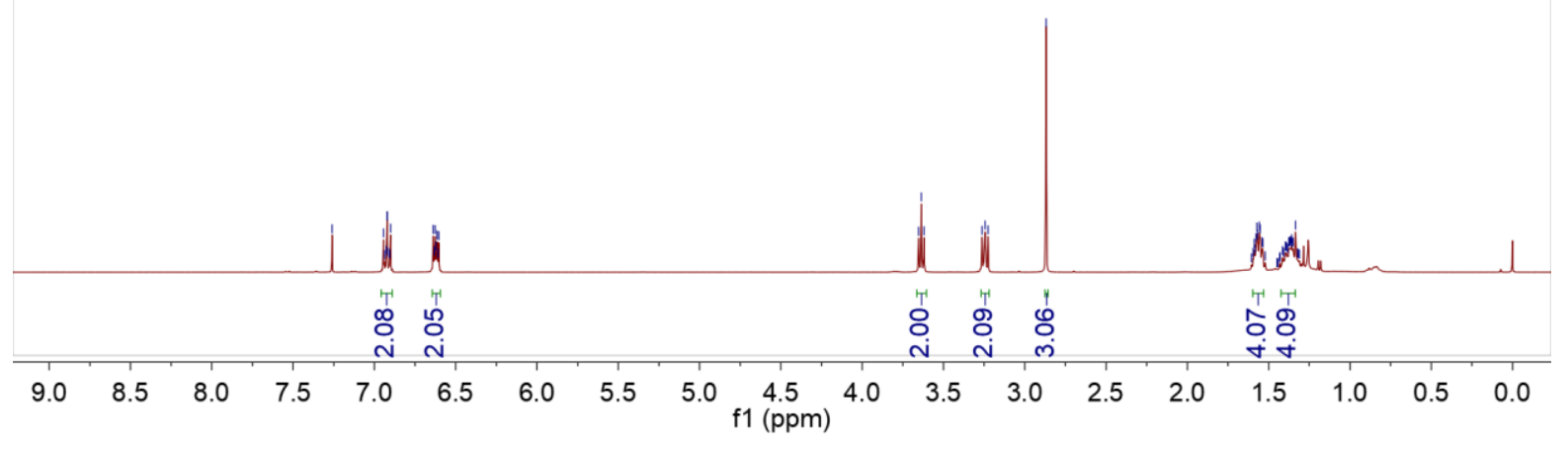




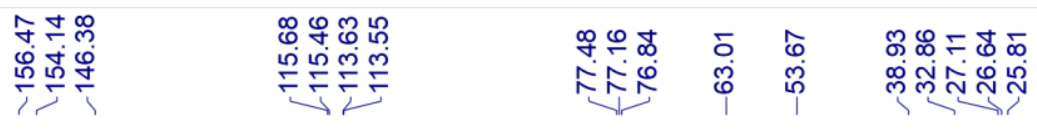

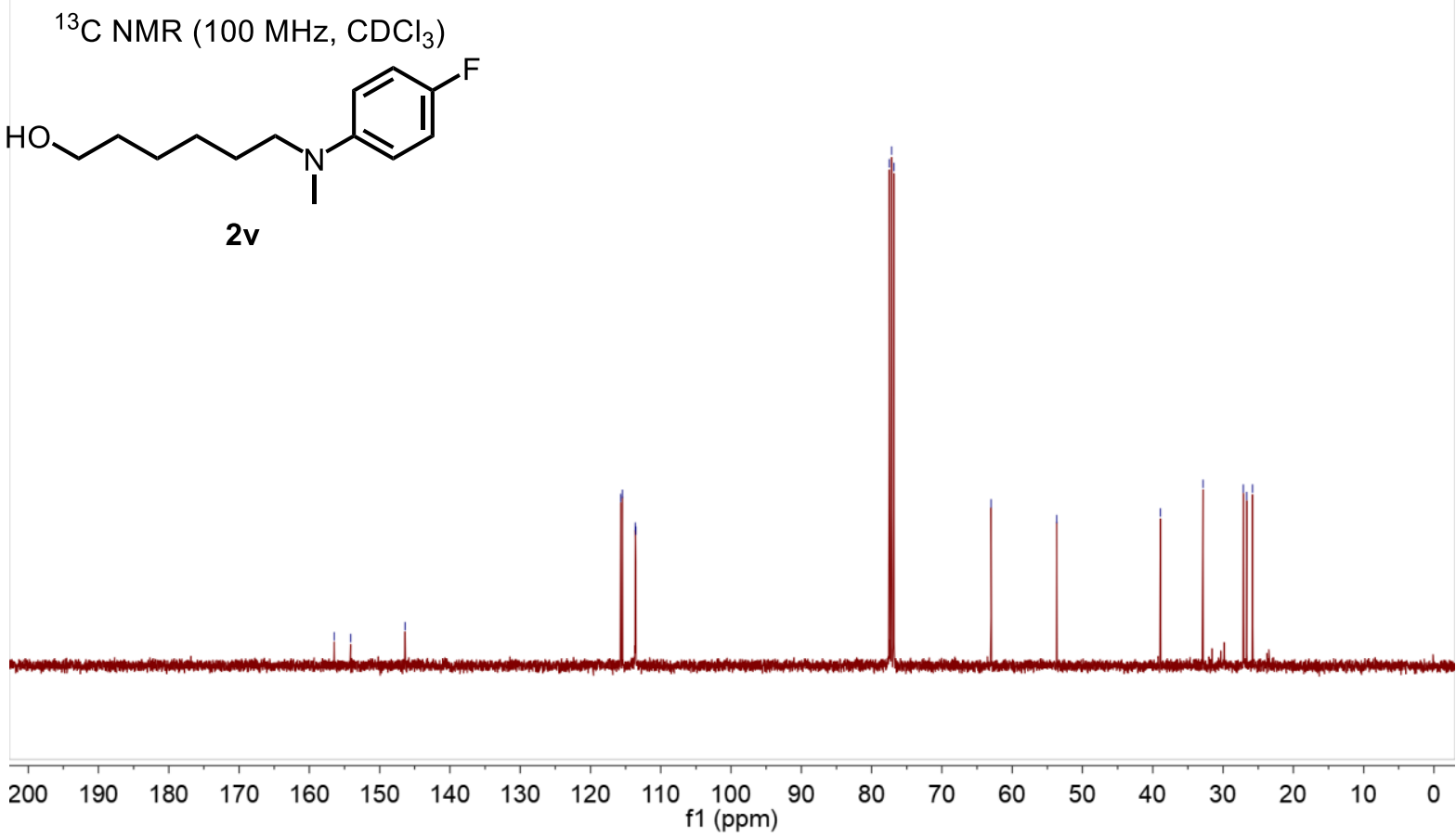

${ }^{19} \mathrm{~F}$ NMR $\left(377 \mathrm{MHz}, \mathrm{CDCl}_{3}\right)$<smiles>CN(CCCCCCO)c1ccc(F)cc1</smiles>

2v

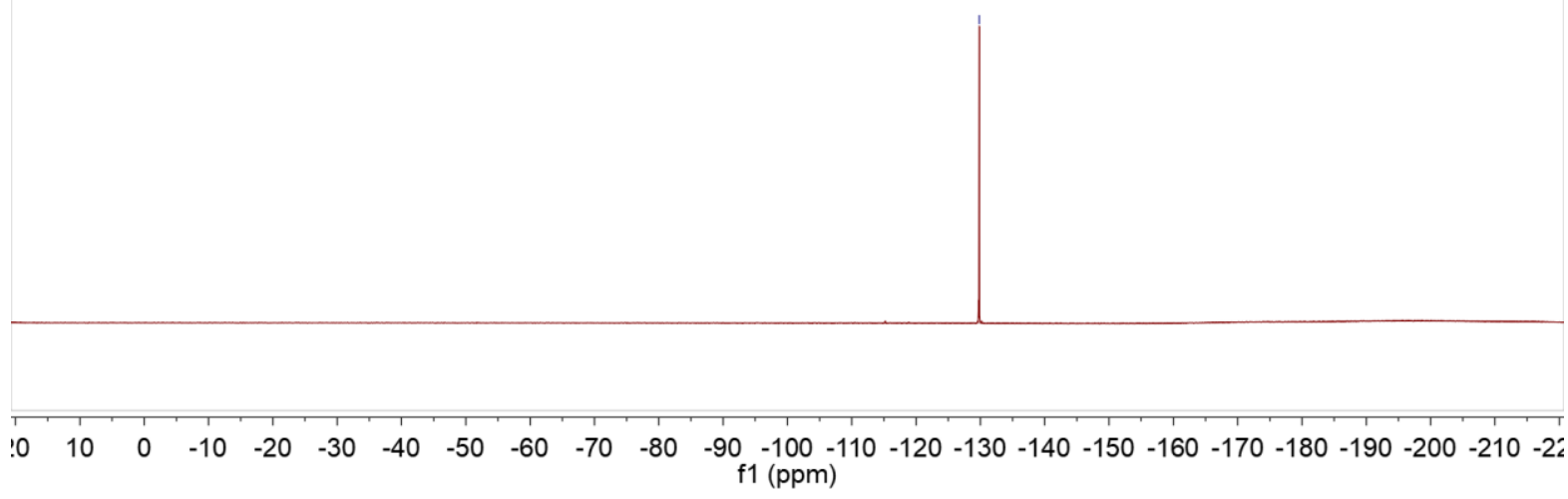


<smiles>CN(CCCCCCO)c1ccc(Cl)cc1</smiles>

$2 w$

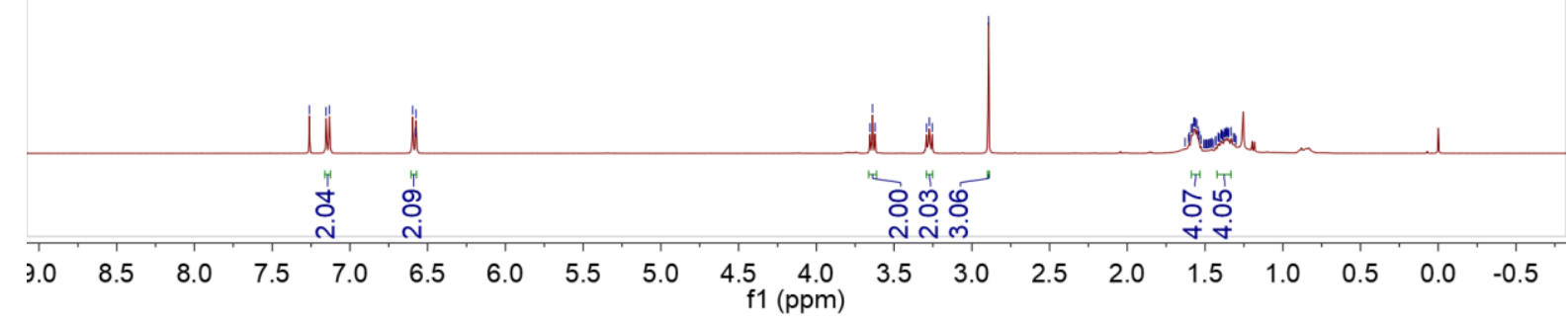

\begin{tabular}{|c|c|c|c|c|}
\hline 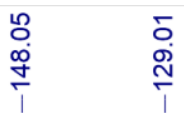 & 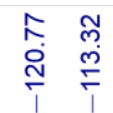 & 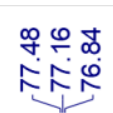 & ర్ & in \\
\hline
\end{tabular}<smiles>CN(CCCCCCO)c1ccc(Cl)cc1</smiles>

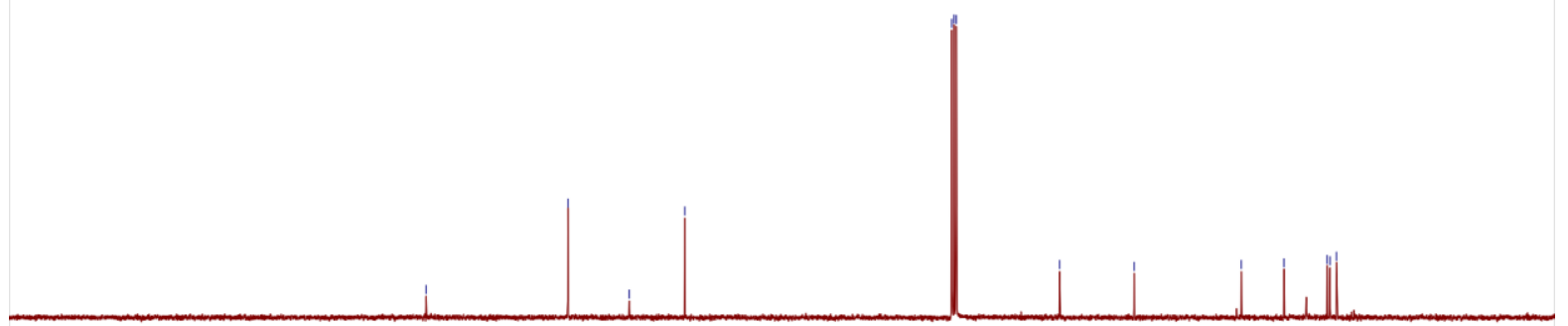

$\begin{array}{lllllllllllllllllllll}200 & 190 & 180 & 170 & 160 & 150 & 140 & 130 & 120 & 110 \underset{\mathrm{f} 1(\mathrm{ppm})}{100} & 90 & 80 & 70 & 60 & 50 & 40 & 30 & 20 & 10 & 0\end{array}$ 
${ }^{1} \mathrm{H}$ NMR $\left(400 \mathrm{MHz}, \mathrm{CDCl}_{3}\right)$<smiles>[R]C(C)(C)c1ccc(N(C)CCCCCCO)cc1</smiles>

$2 x$

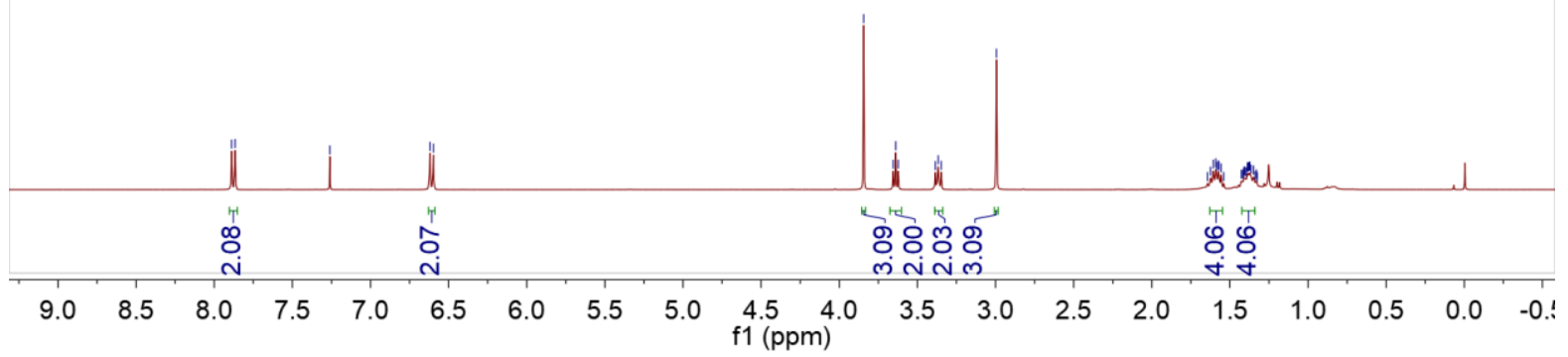

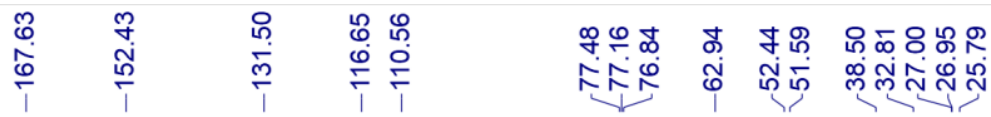
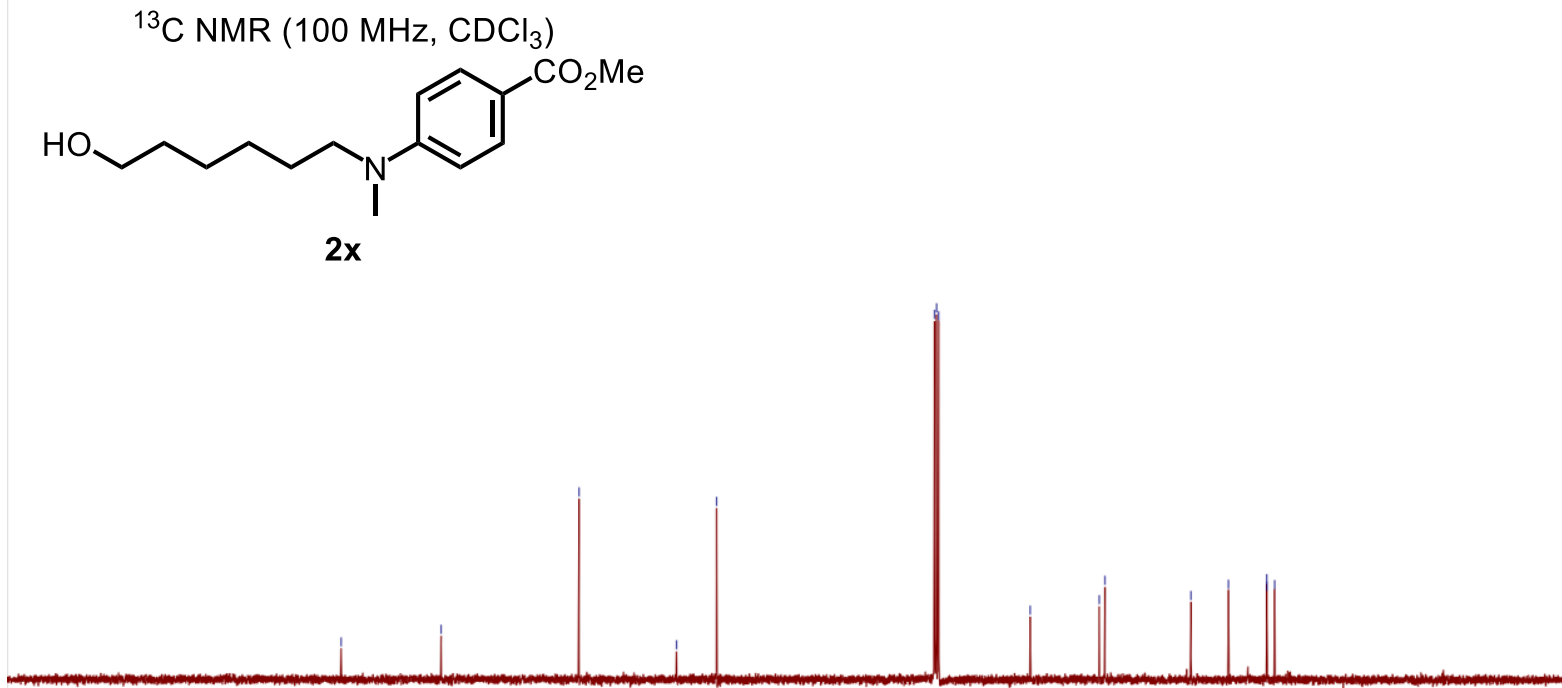

$\begin{array}{lllllllllllllllllllllll}210 & 200 & 190 & 180 & 170 & 160 & 150 & 140 & 130 & 120 & \begin{array}{c}110 \\ \mathrm{f} 1\end{array} \mathrm{fpm}_{(\mathrm{ppm})}^{100} & 90 & 80 & 70 & 60 & 50 & 40 & 30 & 20 & 10 & 0 & -10\end{array}$ 
${ }^{1} \mathrm{H}$ NMR $\left(400 \mathrm{MHz}, \mathrm{CDCl}_{3}\right)$<smiles>OCCCCCCN1CCCc2ccccc21</smiles>

2y

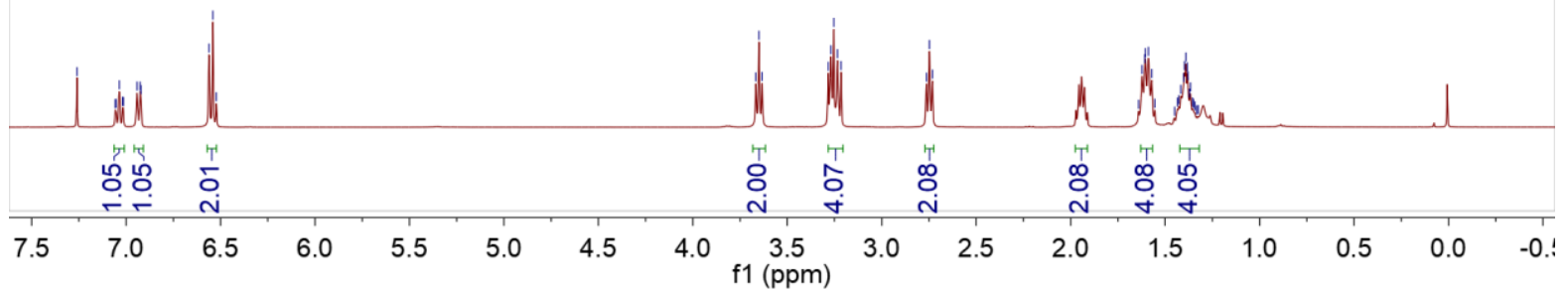

\begin{tabular}{|c|c|c|c|c|}
\hline 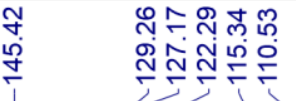 & 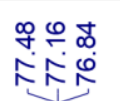 & $\begin{array}{l}\mathscr{8} \\
0 \\
0\end{array}$ & 贠 & 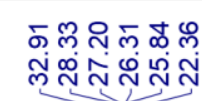 \\
\hline
\end{tabular}

${ }^{13} \mathrm{C}$ NMR $\left(100 \mathrm{MHz}, \mathrm{CDCl}_{3}\right)$<smiles>OCCCCCCN1CCCc2ccccc21</smiles>

$2 y$

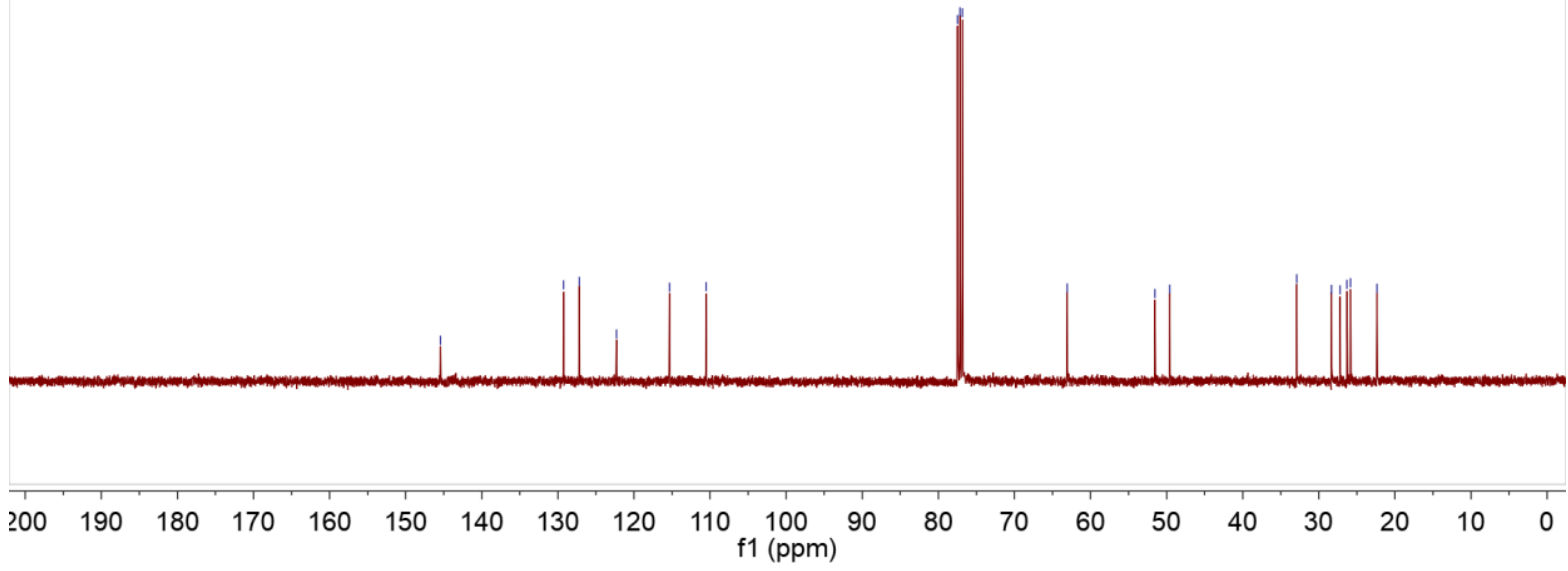


${ }^{1} \mathrm{H}$ NMR $\left(400 \mathrm{MHz}, \mathrm{CDCl}_{3}\right)$<smiles>OCCCCCCN(c1ccccc1)c1ccccc1</smiles>

$2 z$

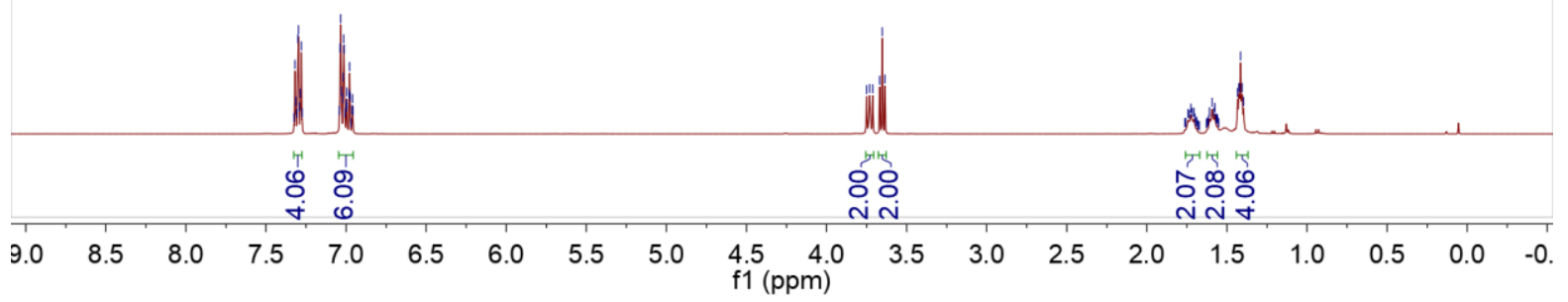

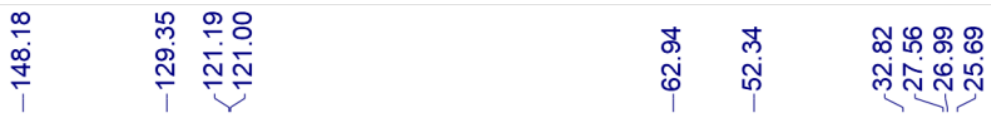

${ }^{13} \mathrm{C}$ NMR $\left(100 \mathrm{MHz}, \mathrm{CDCl}_{3}\right)$<smiles>OCCCCCCN(c1ccccc1)c1ccccc1</smiles>

$2 z$

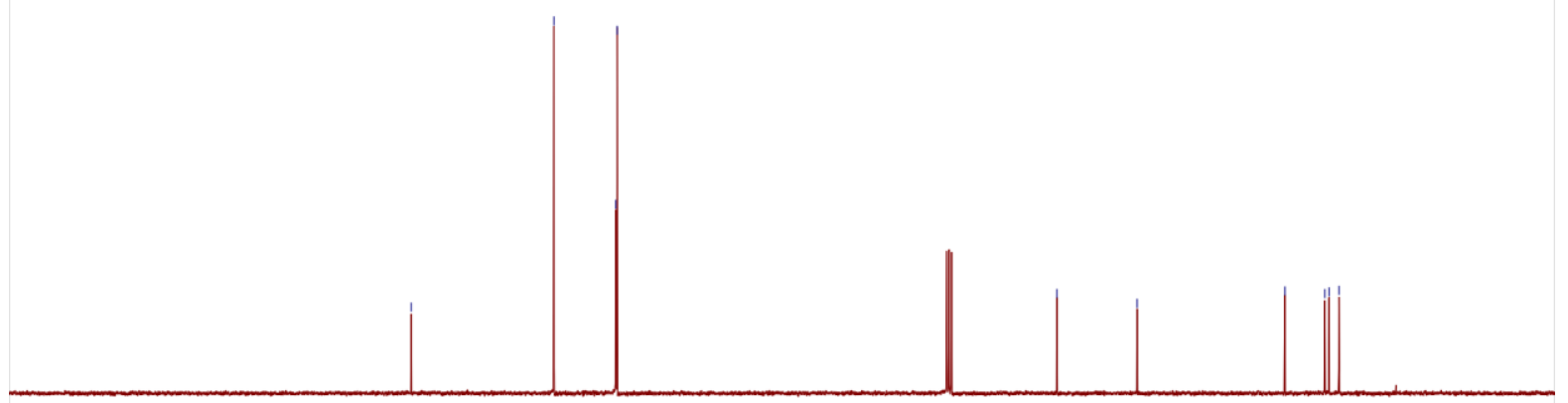

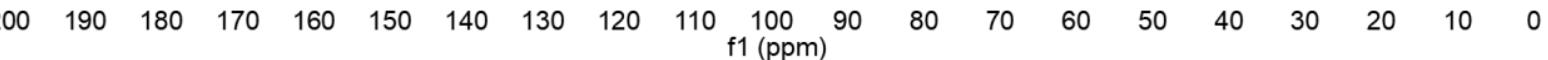


${ }^{1} \mathrm{H} \mathrm{NMR}\left(400 \mathrm{MHz}, \mathrm{CDCl}_{3}\right)$

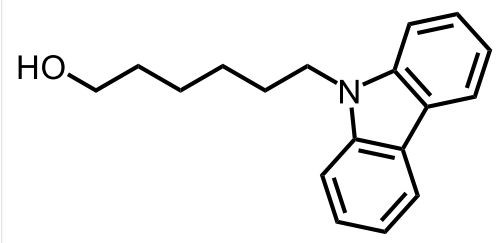

$2 \mathrm{aa}$

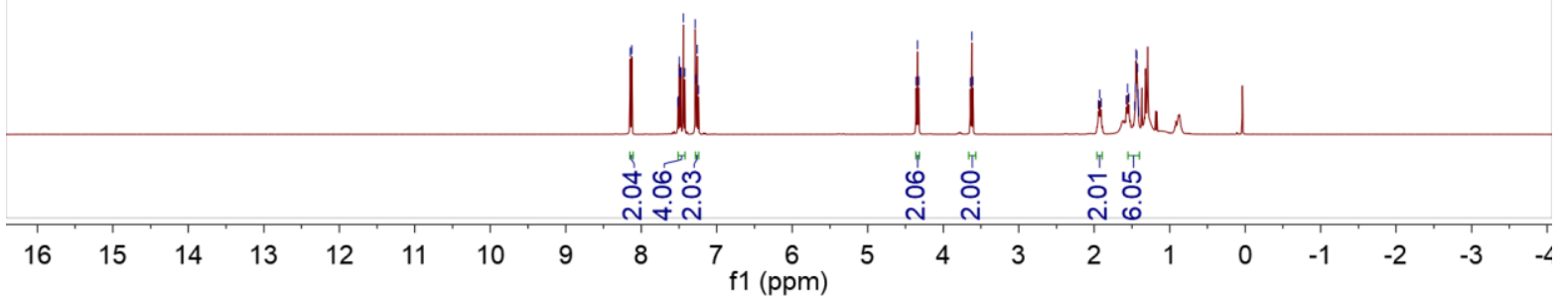

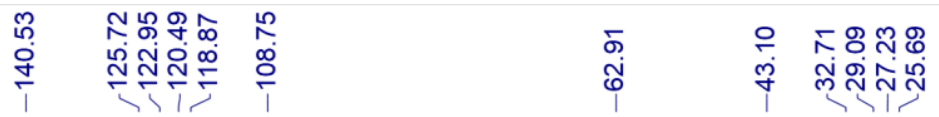

${ }^{13} \mathrm{C}$ NMR $\left(100 \mathrm{MHz}, \mathrm{CDCl}_{3}\right)$<smiles>OCCCCCCn1c2ccccc2c2ccccc21</smiles>

2aa

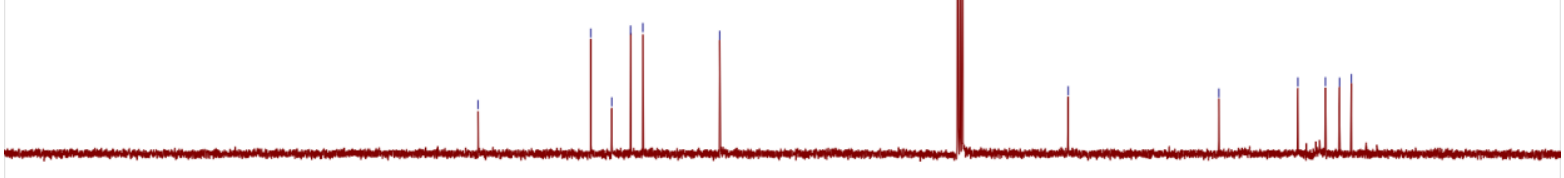

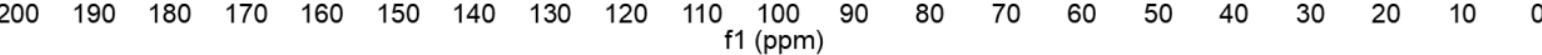


${ }^{1} \mathrm{H} \mathrm{NMR}\left(400 \mathrm{MHz}, \mathrm{CDCl}_{3}\right)$

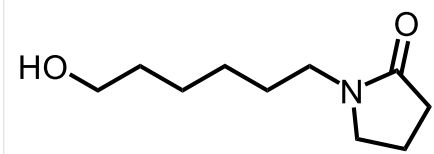

$2 a b$

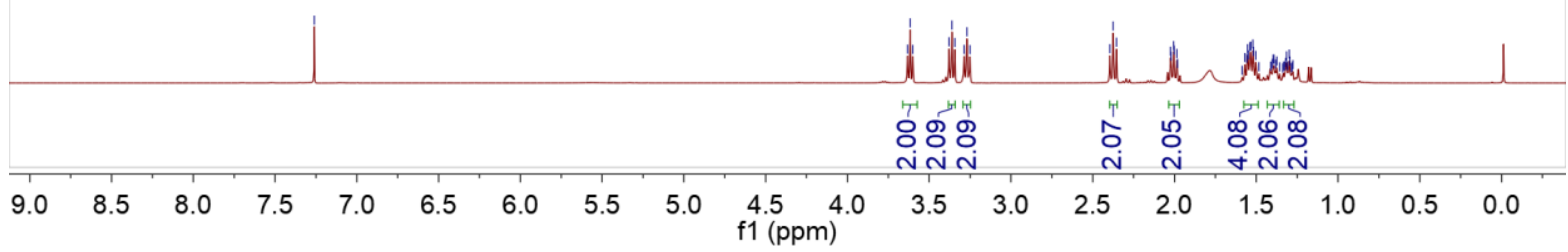

\begin{tabular}{|c|c|c|}
\hline 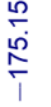 & 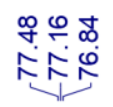 & $\begin{array}{l}\emptyset \\
\text { ஸें } \\
\text { । }\end{array}$ \\
\hline
\end{tabular}

${ }^{13} \mathrm{C}$ NMR $\left(100 \mathrm{MHz}, \mathrm{CDCl}_{3}\right)$

幽

$2 a b$

$\begin{array}{lllllllllllllllllllll}200 & 190 & 180 & 170 & 160 & 150 & 140 & 130 & 120 & 110 & 100 & 90 & 80 & 70 & 60 & 50 & 40 & 30 & 20 & 10 & 0\end{array}$ 
${ }^{1} \mathrm{H}$ NMR $\left(400 \mathrm{MHz}, \mathrm{CDCl}_{3}\right)$<smiles>CC(C)(C)OC(=O)N(CCCCCO)CCCCCCO</smiles>

$2 a c$

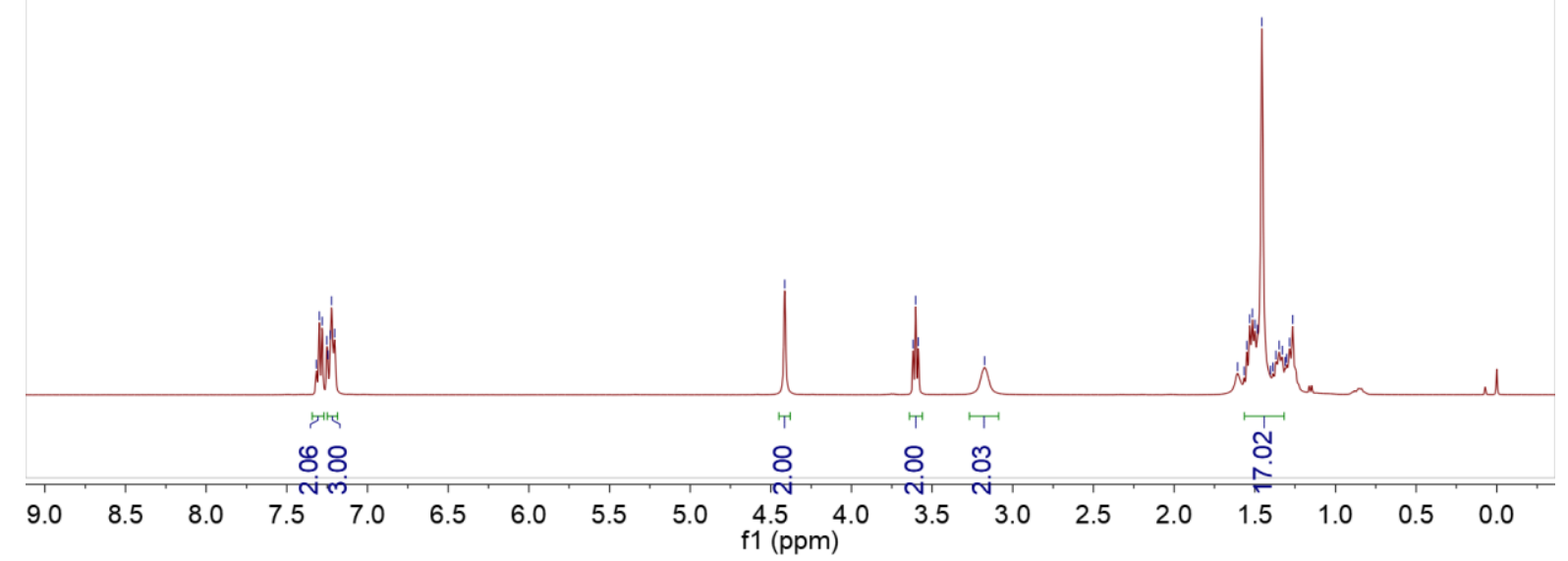

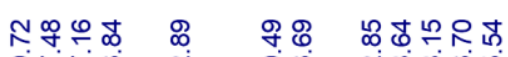

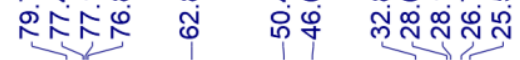

${ }^{13} \mathrm{C}$ NMR $\left(100 \mathrm{MHz}, \mathrm{CDCl}_{3}\right)$<smiles>CC(C)(C)OC(=O)N(CCCCCCO)Cc1ccccc1</smiles>

$2 a c$

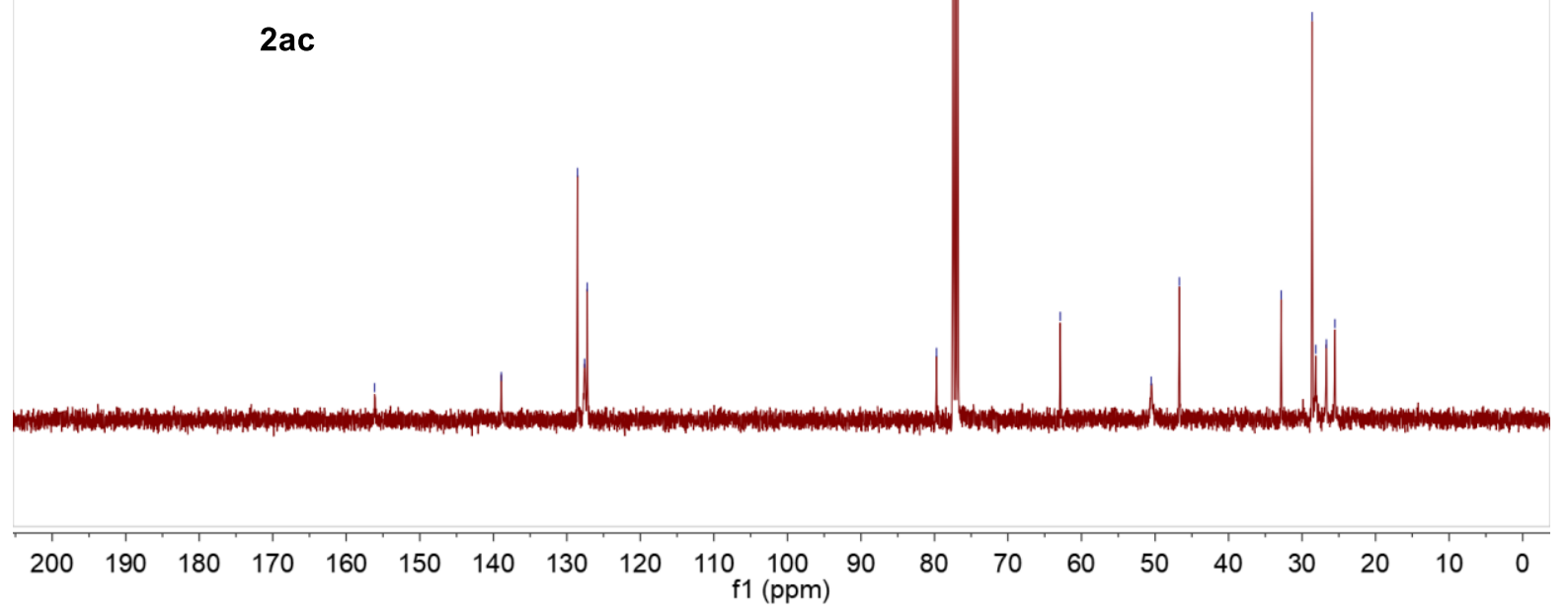


${ }^{1} \mathrm{H}$ NMR $\left(400 \mathrm{MHz}, \mathrm{CDCl}_{3}\right)$<smiles>CC(C)(C)C(=O)N(CCCCCCO)Cc1ccccc1</smiles>

2ad

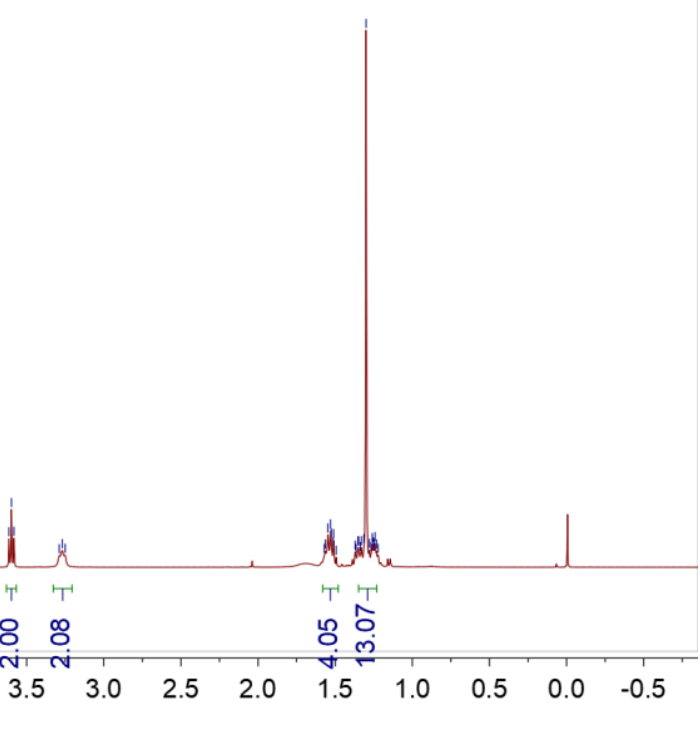

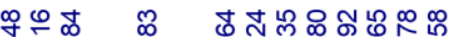

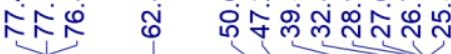

${ }^{13} \mathrm{C}$ NMR $\left(100 \mathrm{MHz}, \mathrm{CDCl}_{3}\right)$

$\mathrm{HO}$<smiles>CCN(Cc1ccccc1)C(=O)C(C)(C)C</smiles>

$2 \mathrm{ad}$

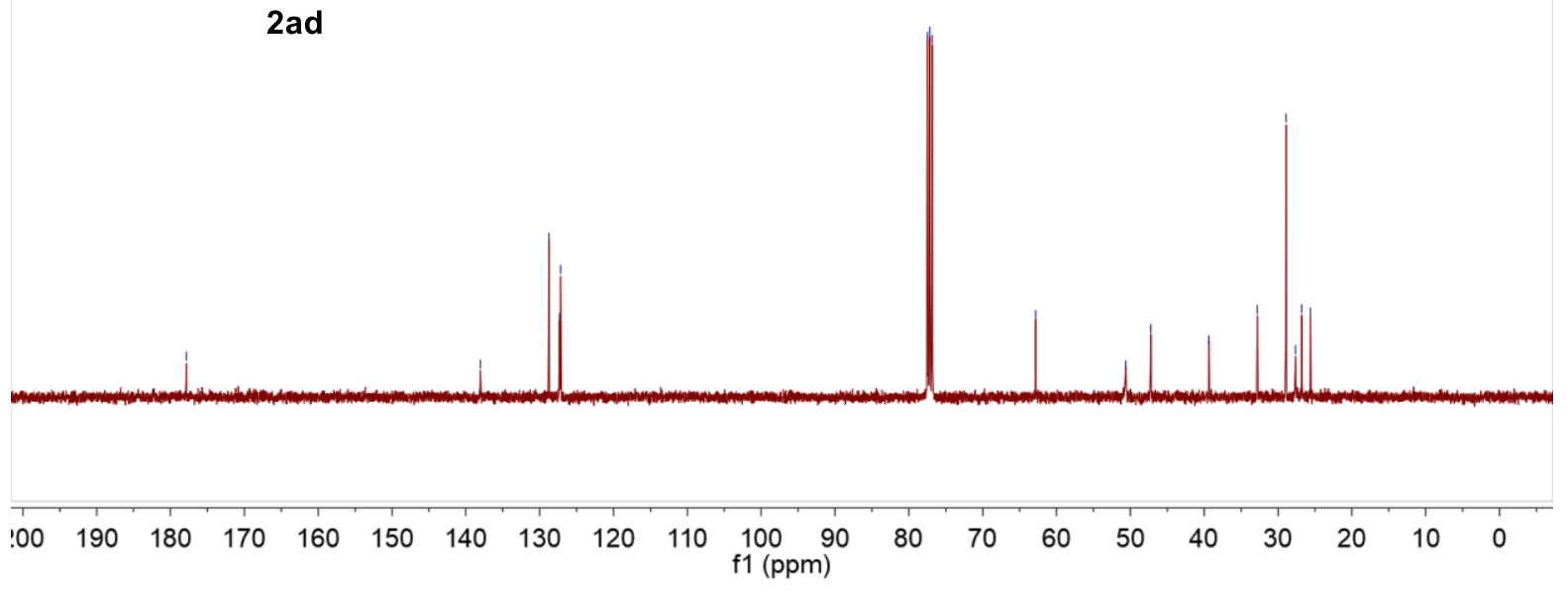


${ }^{1} \mathrm{H}$ NMR $\left(400 \mathrm{MHz}, \mathrm{CDCl}_{3}\right)$

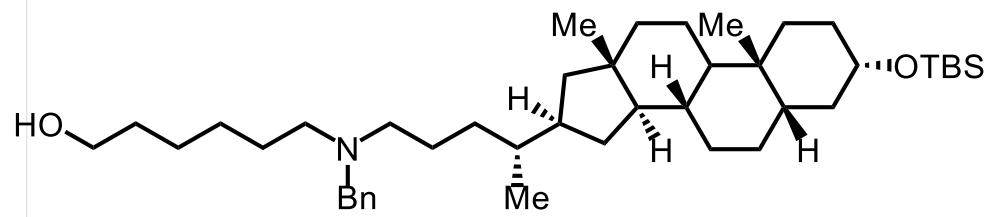

2ae

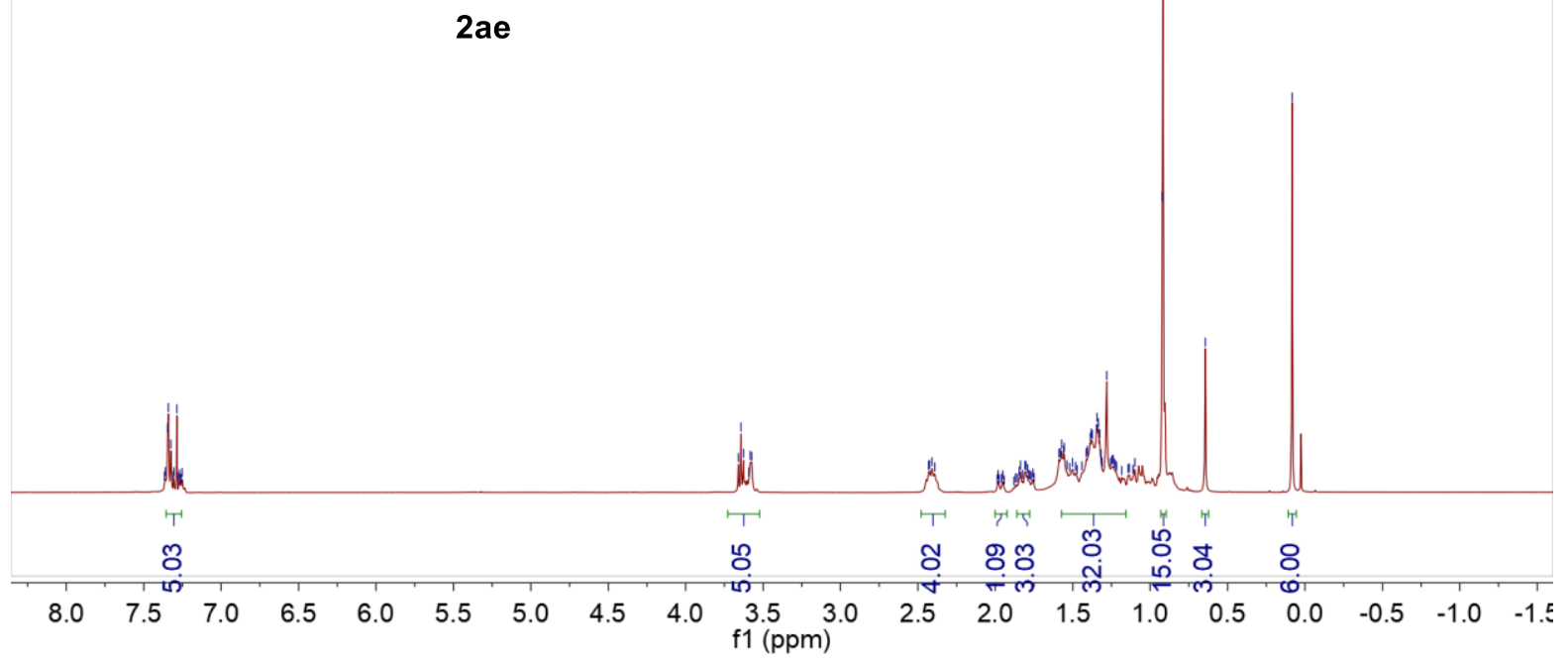

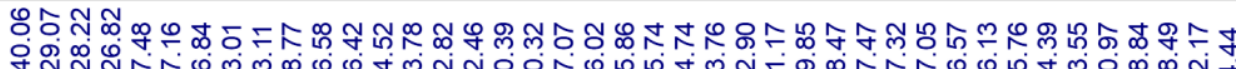

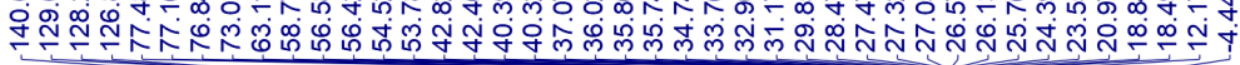

${ }^{13} \mathrm{C}$ NMR $\left(100 \mathrm{MHz}, \mathrm{CDCl}_{3}\right)$

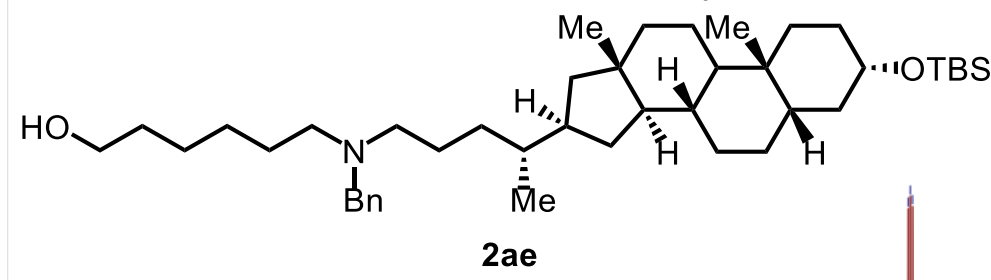

$2 \mathrm{ae}$

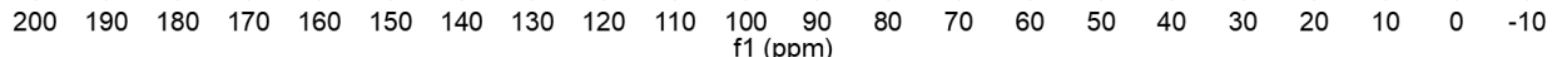




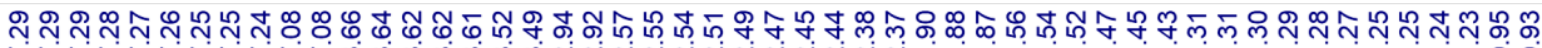

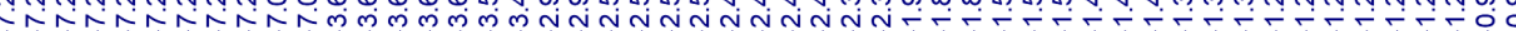

${ }^{1} \mathrm{H}$ NMR $\left(400 \mathrm{MHz}, \mathrm{CDCl}_{3}\right)$<smiles>CC(C)Cc1ccc(C(C)CN(CCCCCCO)Cc2ccccc2)cc1</smiles>

2af

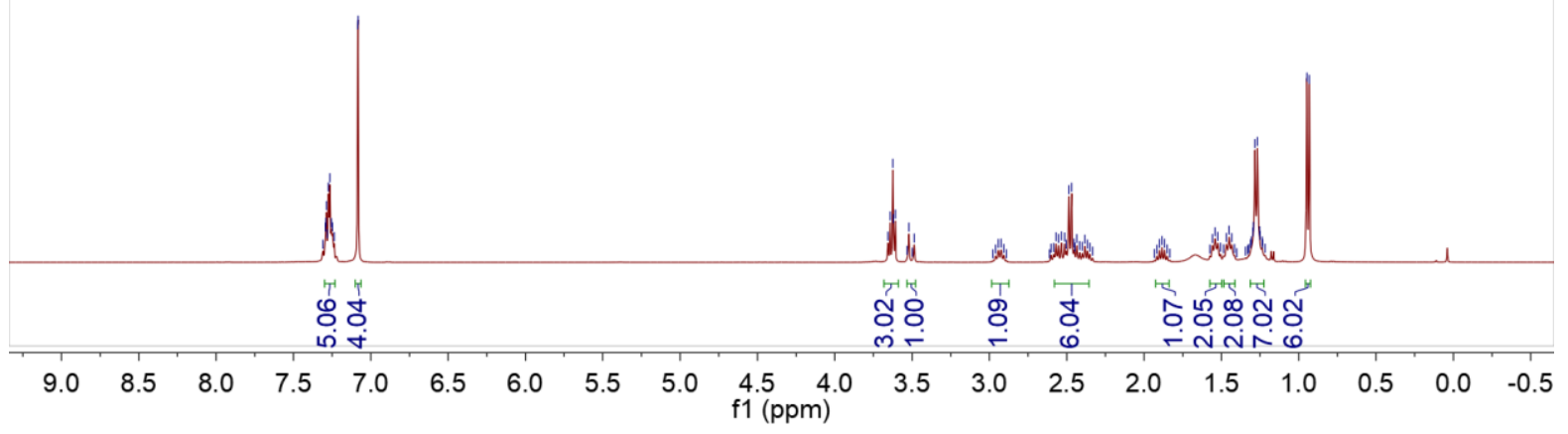

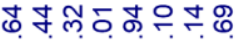

旁

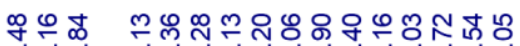

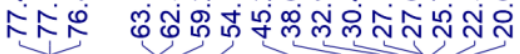

${ }^{13} \mathrm{C}$ NMR $\left(100 \mathrm{MHz}, \mathrm{CDCl}_{3}\right)$<smiles>CC(C)Cc1ccc(C(C)CN(CCCCCCO)Cc2ccccc2)cc1</smiles>

2af

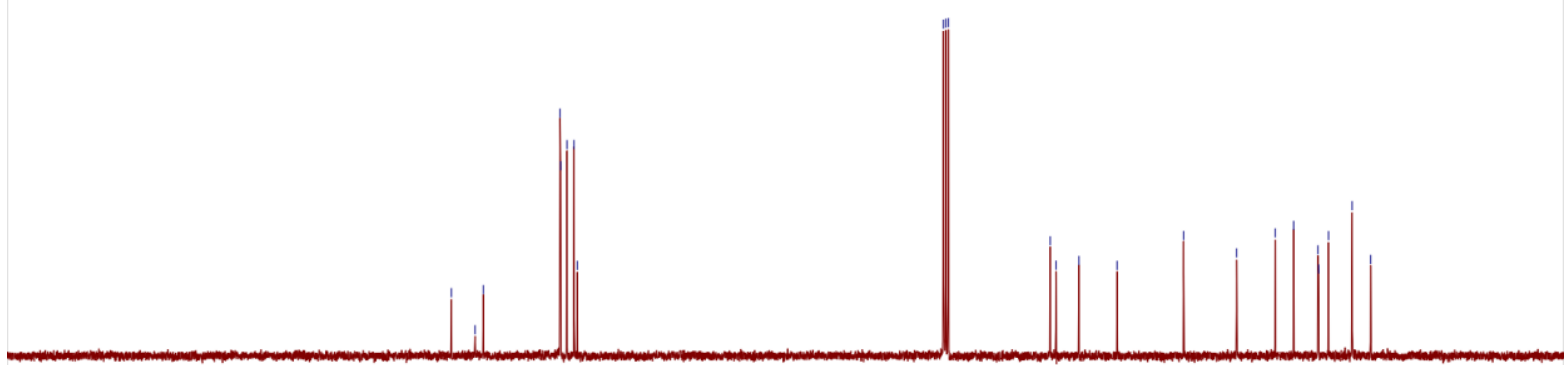


${ }^{1} \mathrm{H} \mathrm{NMR}\left(400 \mathrm{MHz}, \mathrm{CDCl}_{3}\right)$<smiles>COc1ccc2cc([C@@H](C)CN(CCCCCCO)Cc3ccccc3)ccc2c1</smiles>

2ag

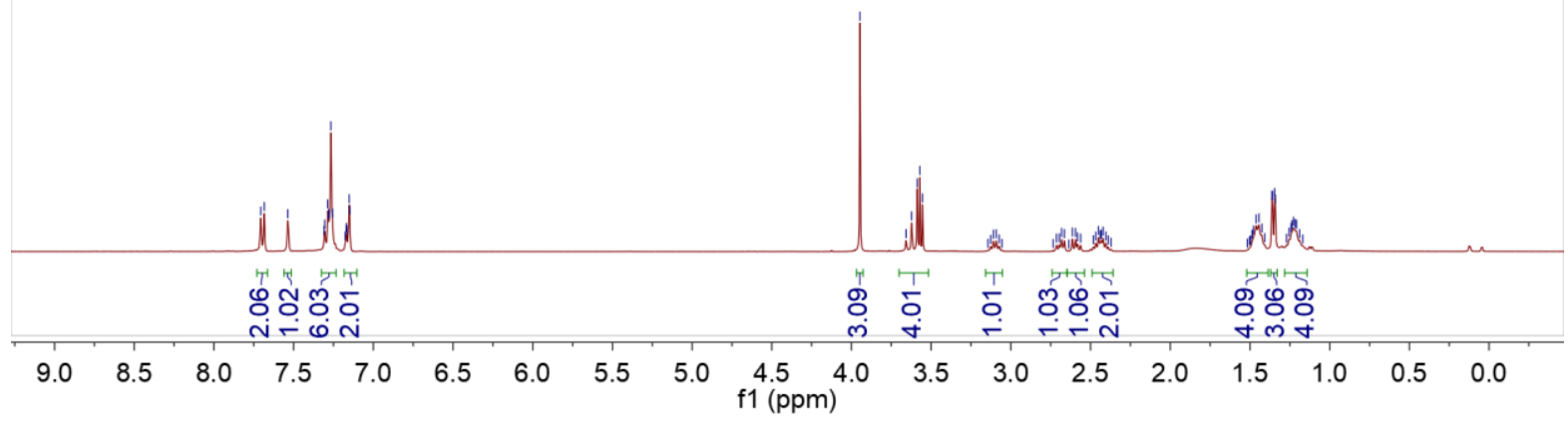

ก்ุ

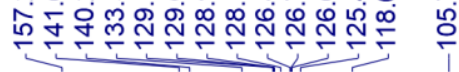

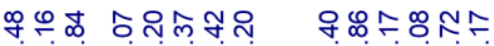

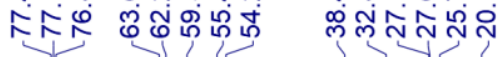

${ }^{13} \mathrm{C}$ NMR $\left(100 \mathrm{MHz}, \mathrm{CDCl}_{3}\right)$<smiles>COc1ccc2cc([C@@H](C)CN(CCCCCCO)Cc3ccccc3)ccc2c1</smiles>

2ag

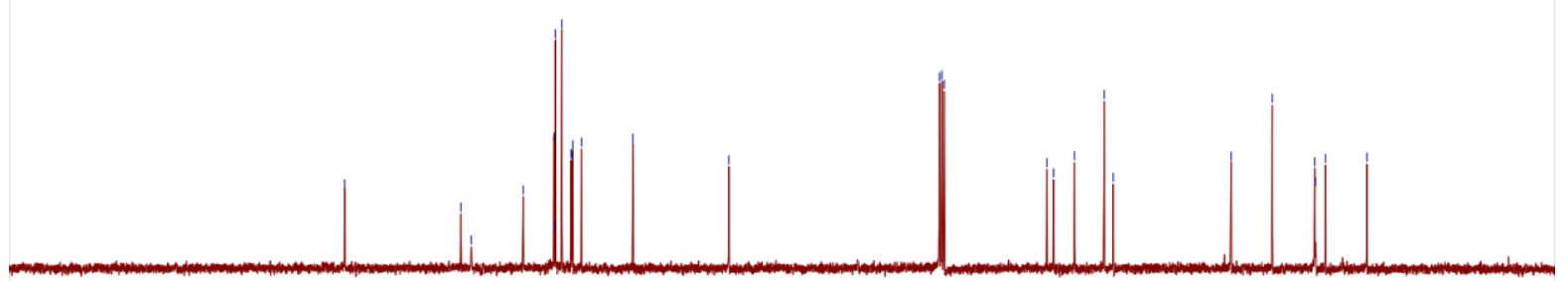

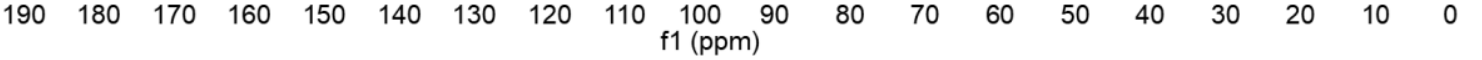


${ }^{1} \mathrm{H}$ NMR $\left(400 \mathrm{MHz}, \mathrm{CDCl}_{3}\right)$

Bpin

$\mathrm{NBn}_{2}$

3a

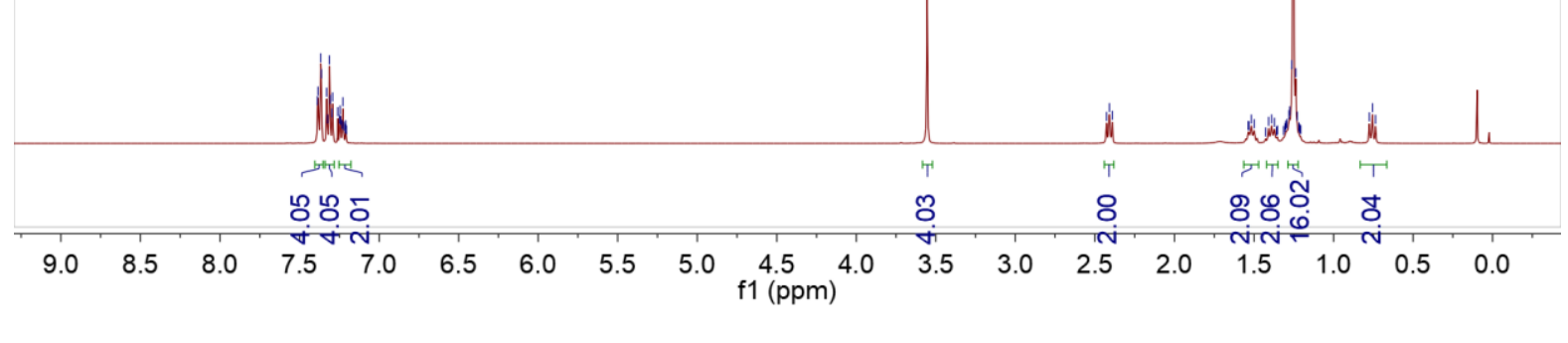

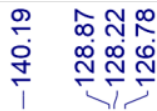

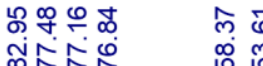

유 츙ำ

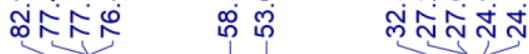

${ }^{13} \mathrm{C}$ NMR $\left(100 \mathrm{MHz}, \mathrm{CDCl}_{3}\right)$

Bpin<smiles>CCCCCCCNC(=O)c1ccccc1</smiles>

3a

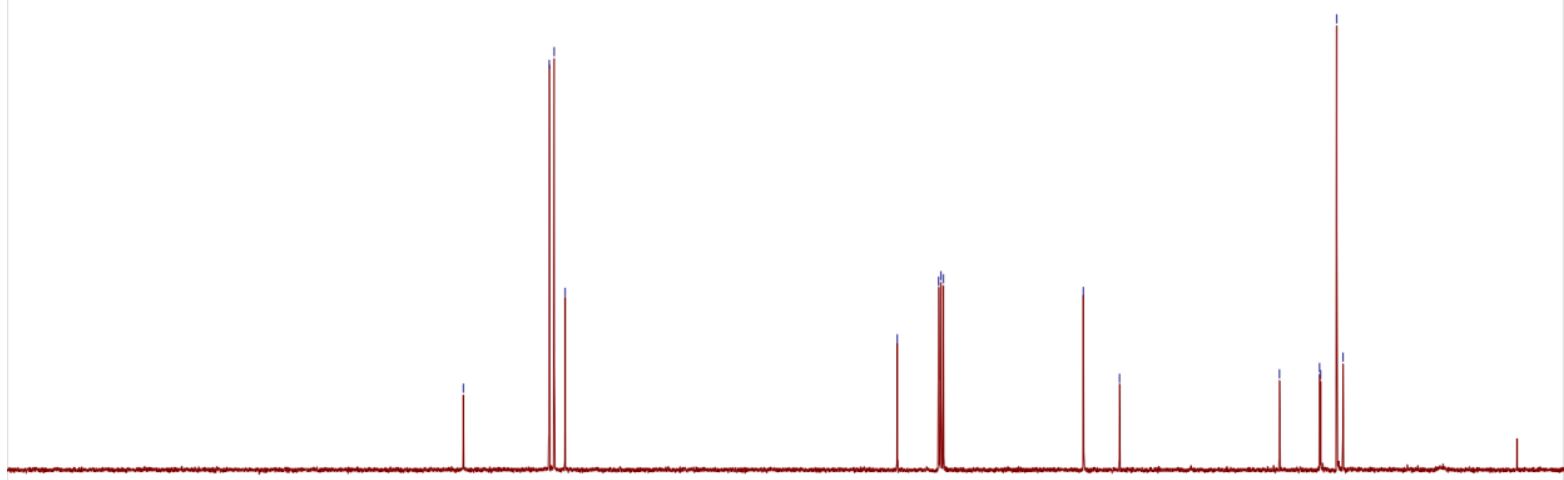

\begin{tabular}{llllllllllllllllllllll}
\hline 0 & 190 & 180 & 170 & 160 & 150 & 140 & 130 & 120 & 110 & $\begin{array}{c}100 \\
\mathrm{f} 1(\mathrm{ppm})\end{array}$ & 80 & 70 & 60 & 50 & 40 & 30 & 20 & 10 & 0
\end{tabular} 


\section{$\underset{\text { F }}{\stackrel{F}{+}}$}

${ }^{11} \mathrm{~B}$ NMR $\left(128 \mathrm{MHz}, \mathrm{CDCl}_{3}\right)$

Bpin $\mathrm{NBn}_{2}$

3a
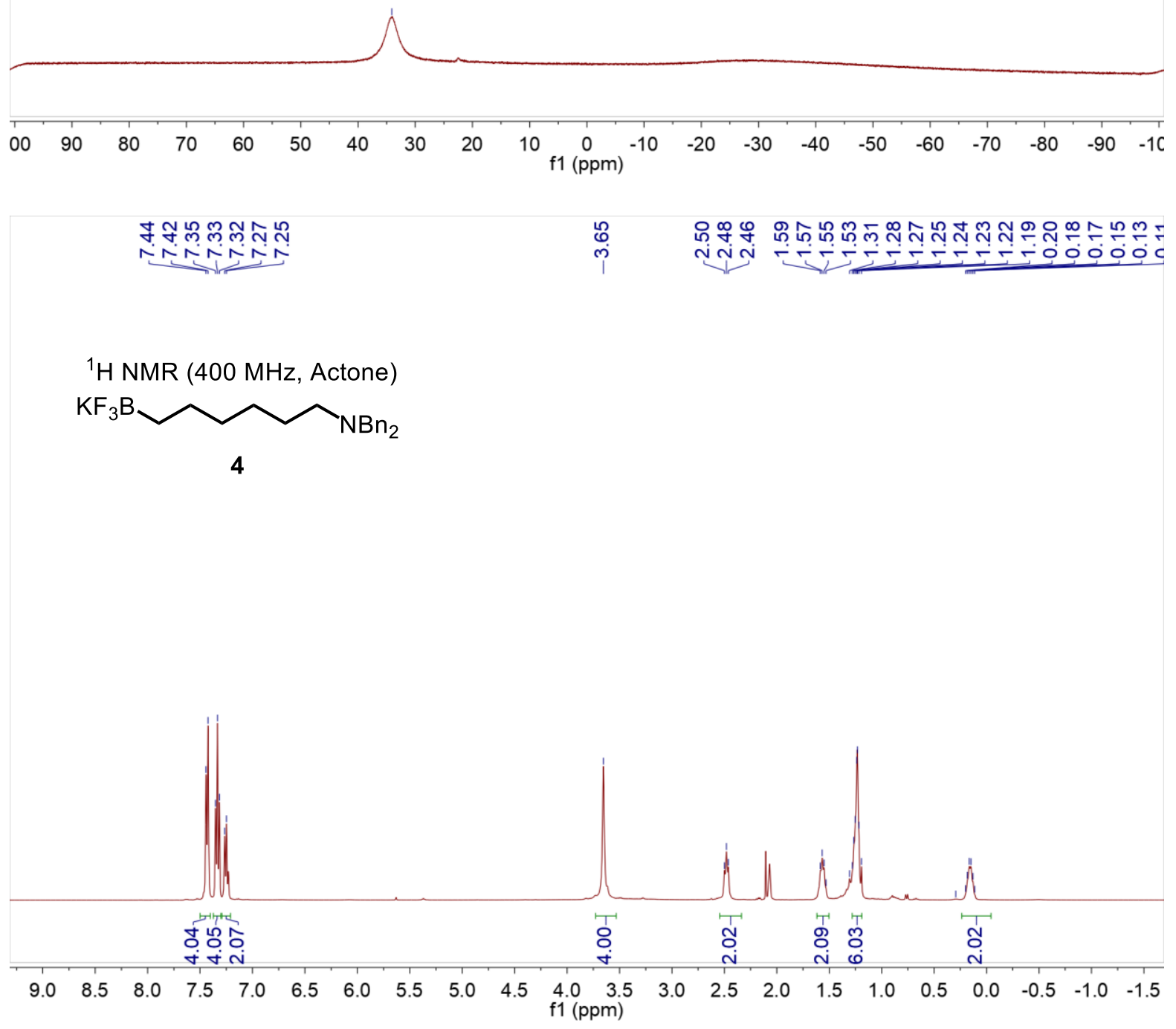
${ }^{13} \mathrm{C}$ NMR $(100 \mathrm{MHz}$, Actone)

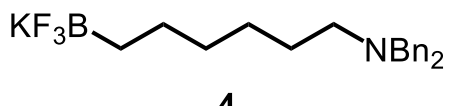

4

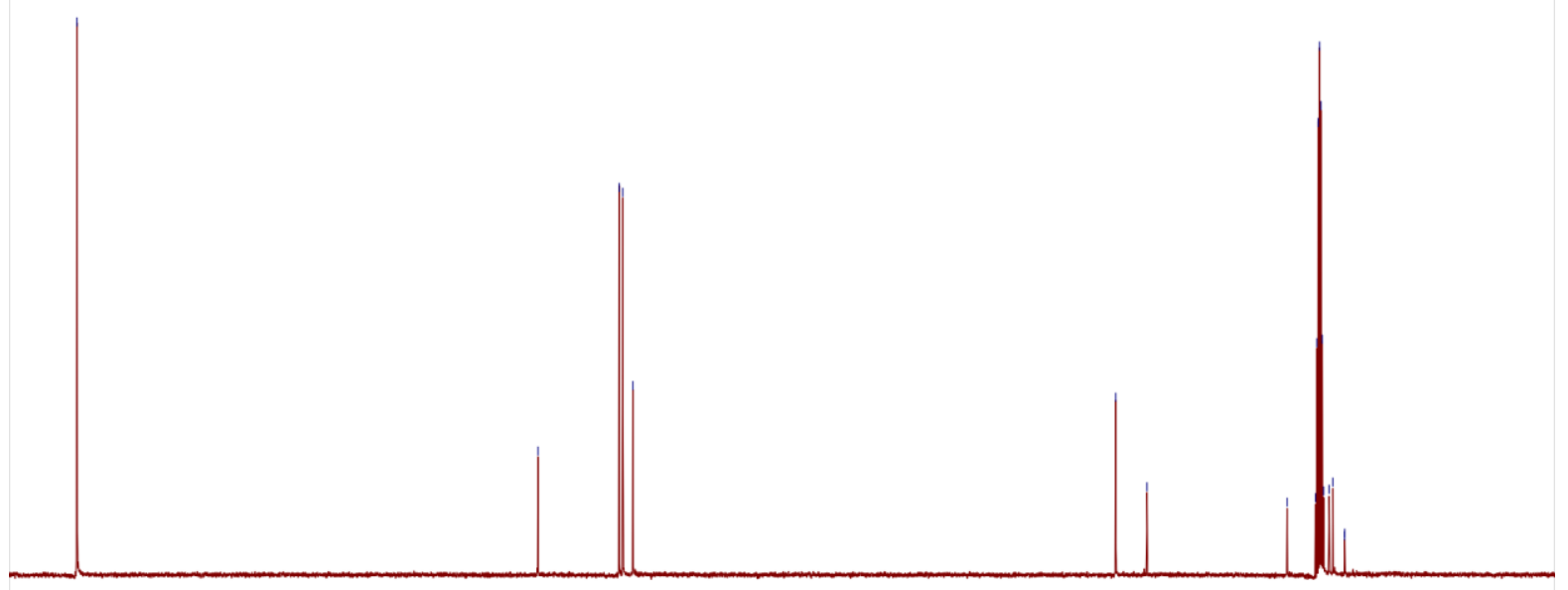

$\begin{array}{llllllllllllllllllllll}210 & 200 & 190 & 180 & 170 & 160 & 150 & 140 & 130 & 120 & \begin{array}{l}110 \\ \mathrm{f} 1(\mathrm{ppm})\end{array} & 90 & 80 & 70 & 60 & 50 & 40 & 30 & 20 & 10 & 0\end{array}$

\section{$\stackrel{\substack{\infty \\ i}}{i}$}

${ }^{11} \mathrm{~B}$ NMR (128 MHz, Actone)

$\mathrm{KF}_{3} \mathrm{~B}$

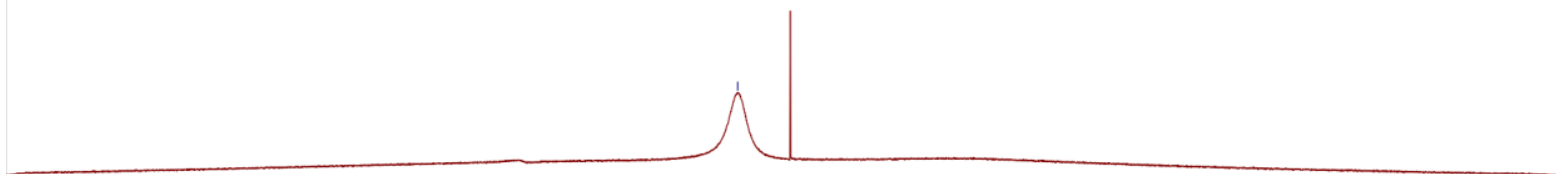

$\begin{array}{llllllllllllllllllll}00 & 90 & 80 & 70 & 60 & 50 & 40 & 30 & 20 & 10 \underset{f 1}{0}(\mathrm{ppm}) & -10 & -20 & -30 & -40 & -50 & -60 & -70 & -80 & -90 & -10\end{array}$ 
${ }^{1} \mathrm{H}$ NMR $\left(400 \mathrm{MHz}, \mathrm{CDCl}_{3}\right)$
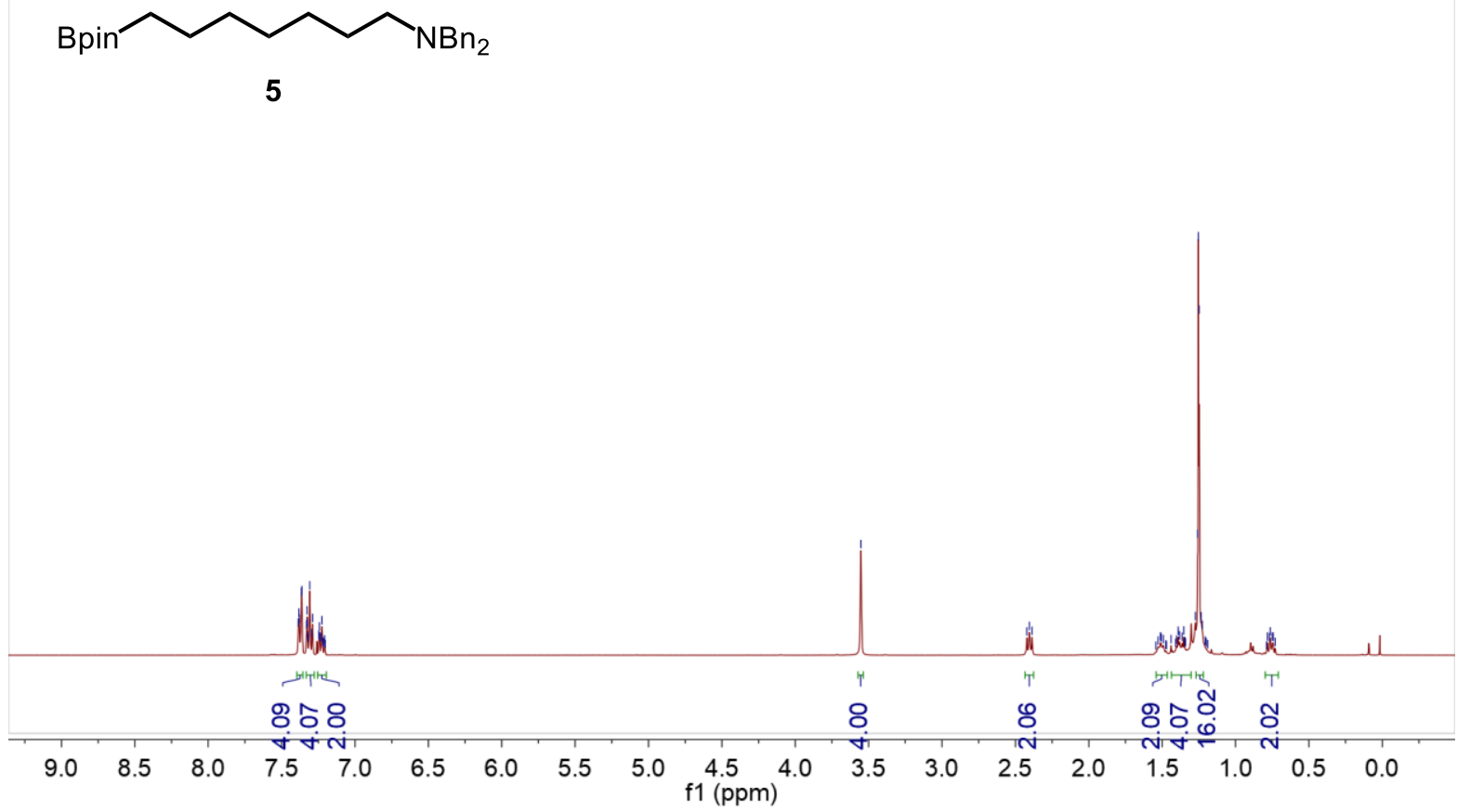

\section{ล่

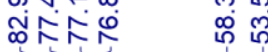 \\ ก เ \\ กี่}

${ }^{13} \mathrm{C}$ NMR $\left(100 \mathrm{MHz}, \mathrm{CDCl}_{3}\right)$

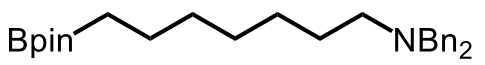

5

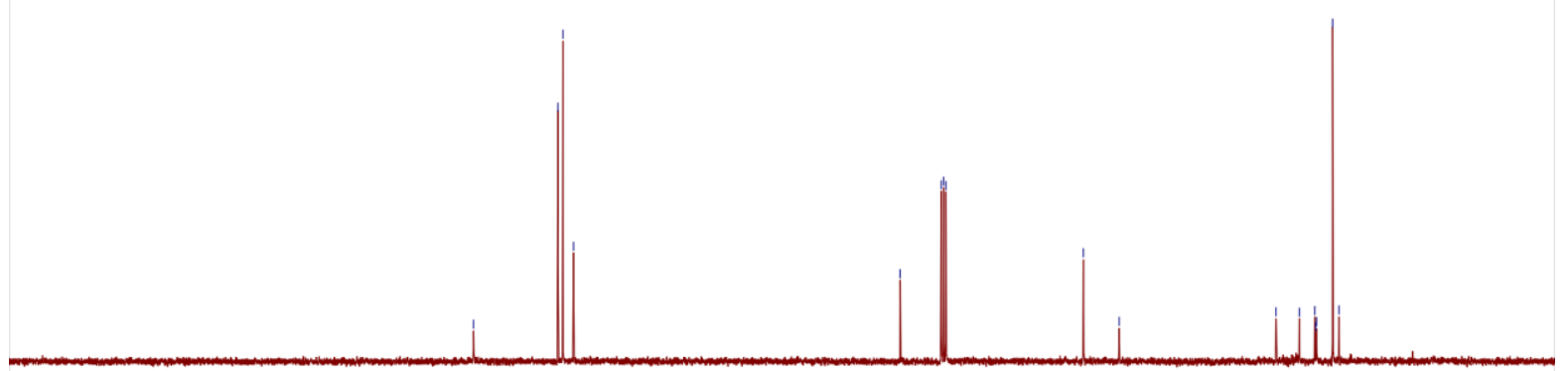




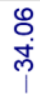

${ }^{11} \mathrm{~B}$ NMR $\left(128 \mathrm{MHz}, \mathrm{CDCl}_{3}\right)$

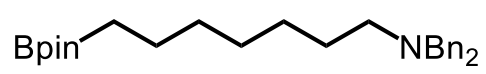

5

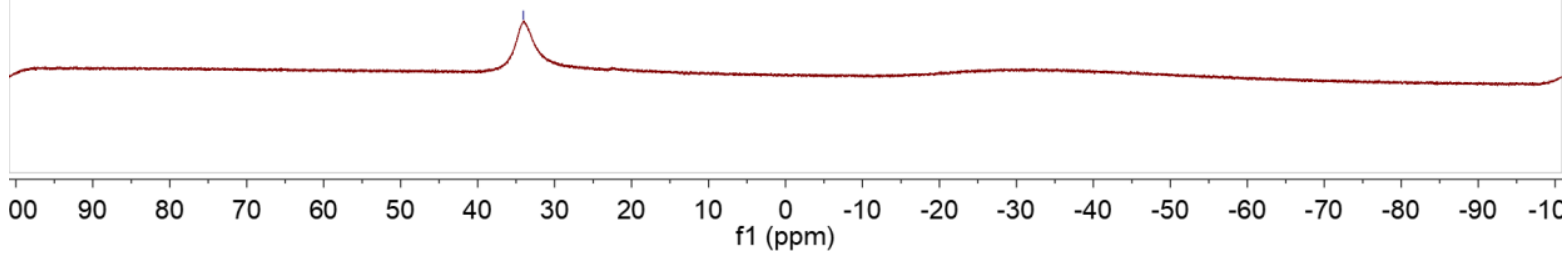

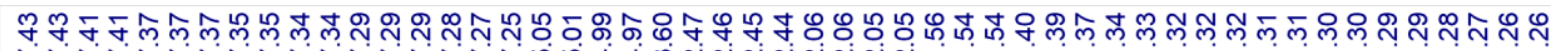

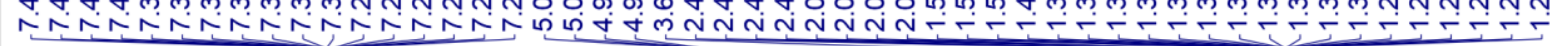

${ }^{1} \mathrm{H}$ NMR $\left(400 \mathrm{MHz}, \mathrm{CDCl}_{3}\right.$ )

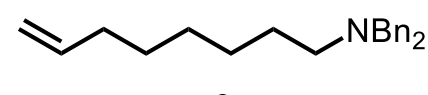

6

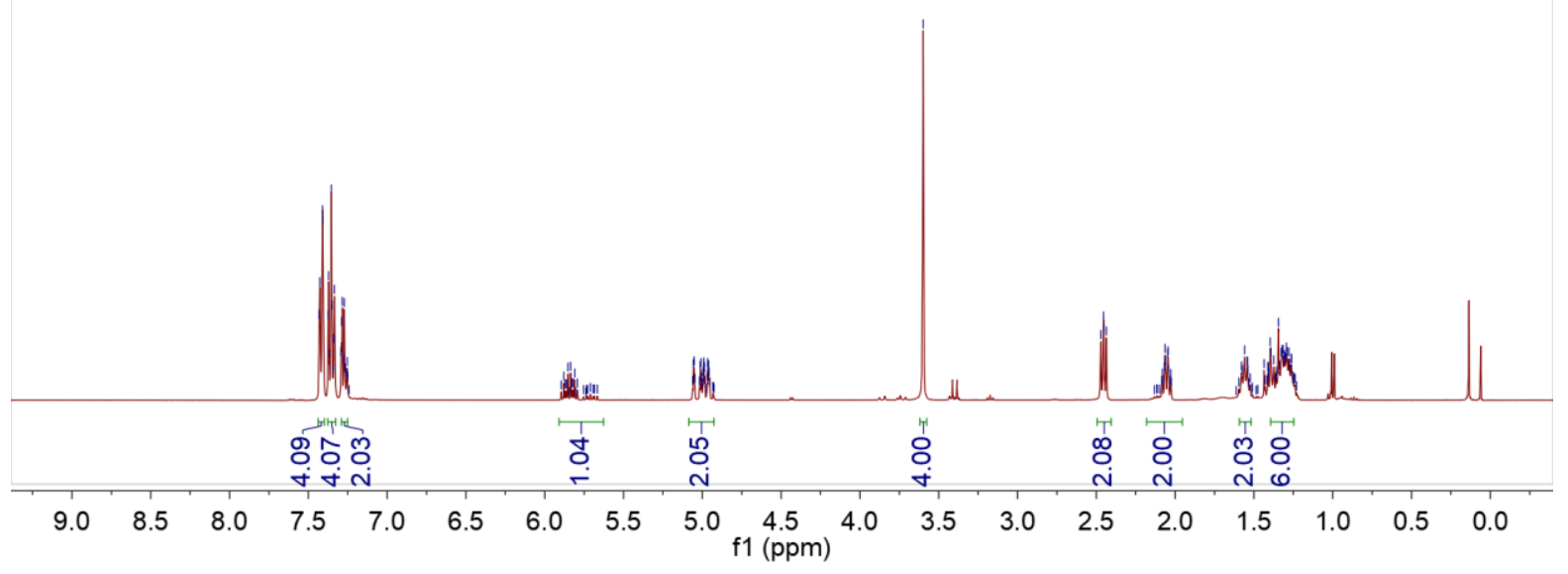




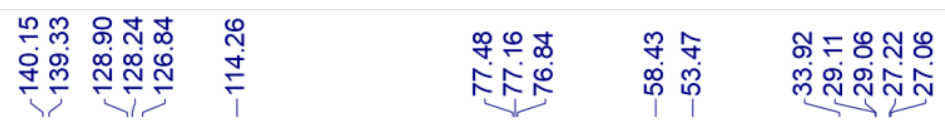

${ }^{13} \mathrm{C}$ NMR $\left(100 \mathrm{MHz}, \mathrm{CDCl}_{3}\right)$

$\overbrace{}^{\mathrm{NBn}_{2}}$

6

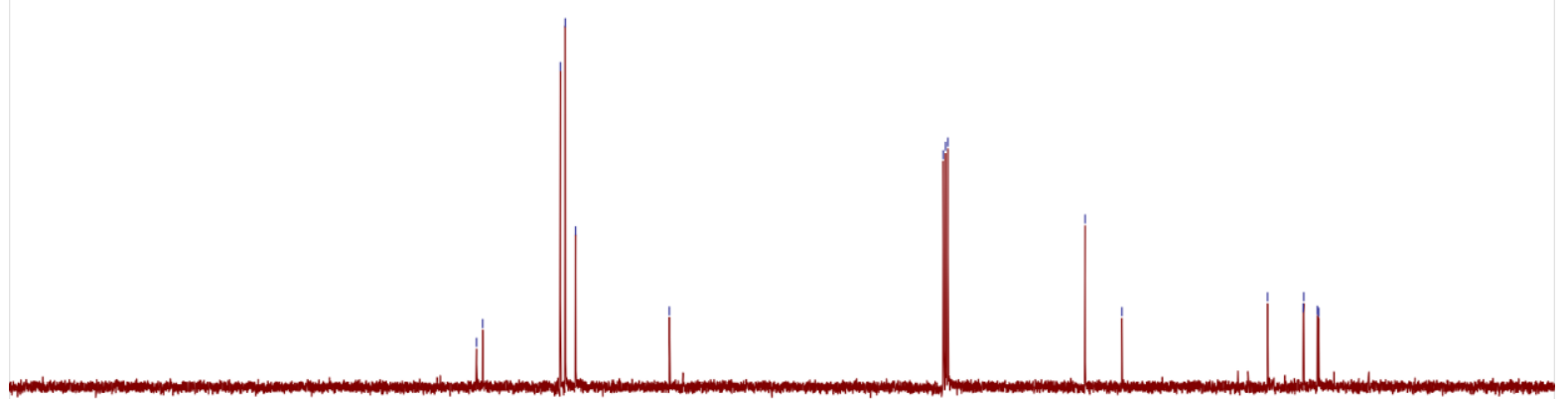

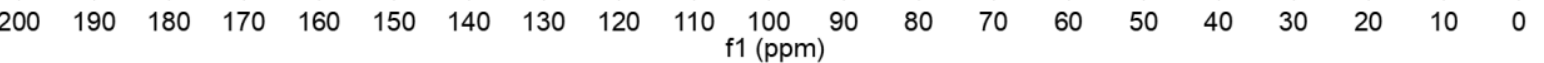

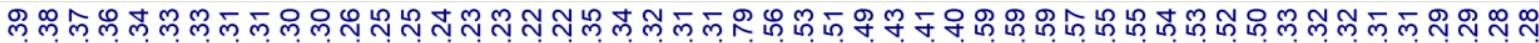

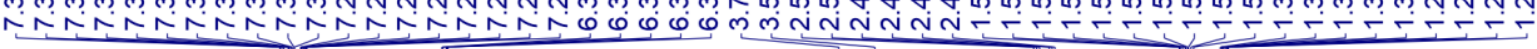<smiles>COc1cc(CCCCCCNC(=O)OCc2ccccc2)cc(OC)c1</smiles>

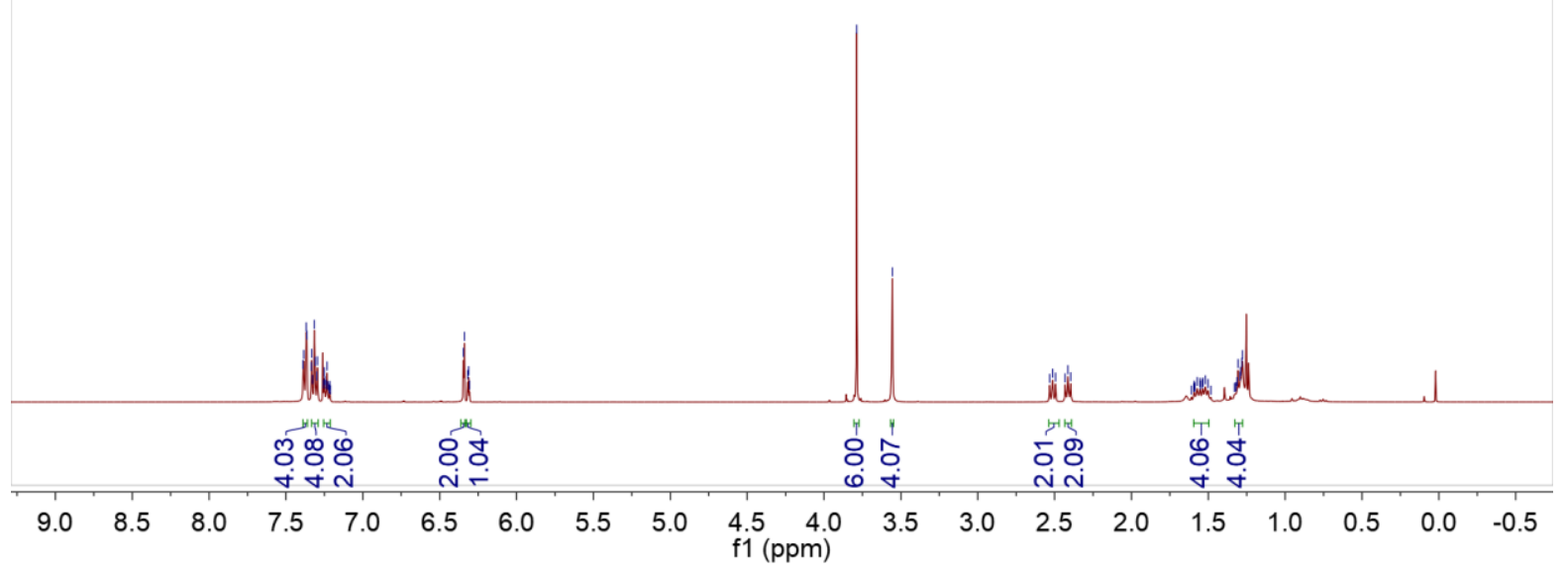




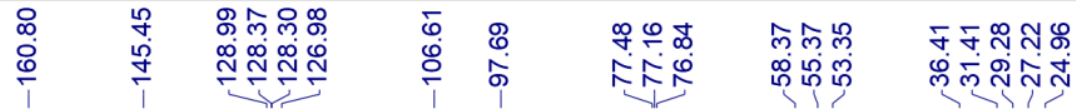

${ }^{13} \mathrm{C} \mathrm{NMR}\left(100 \mathrm{MHz}, \mathrm{CDCl}_{3}\right)$
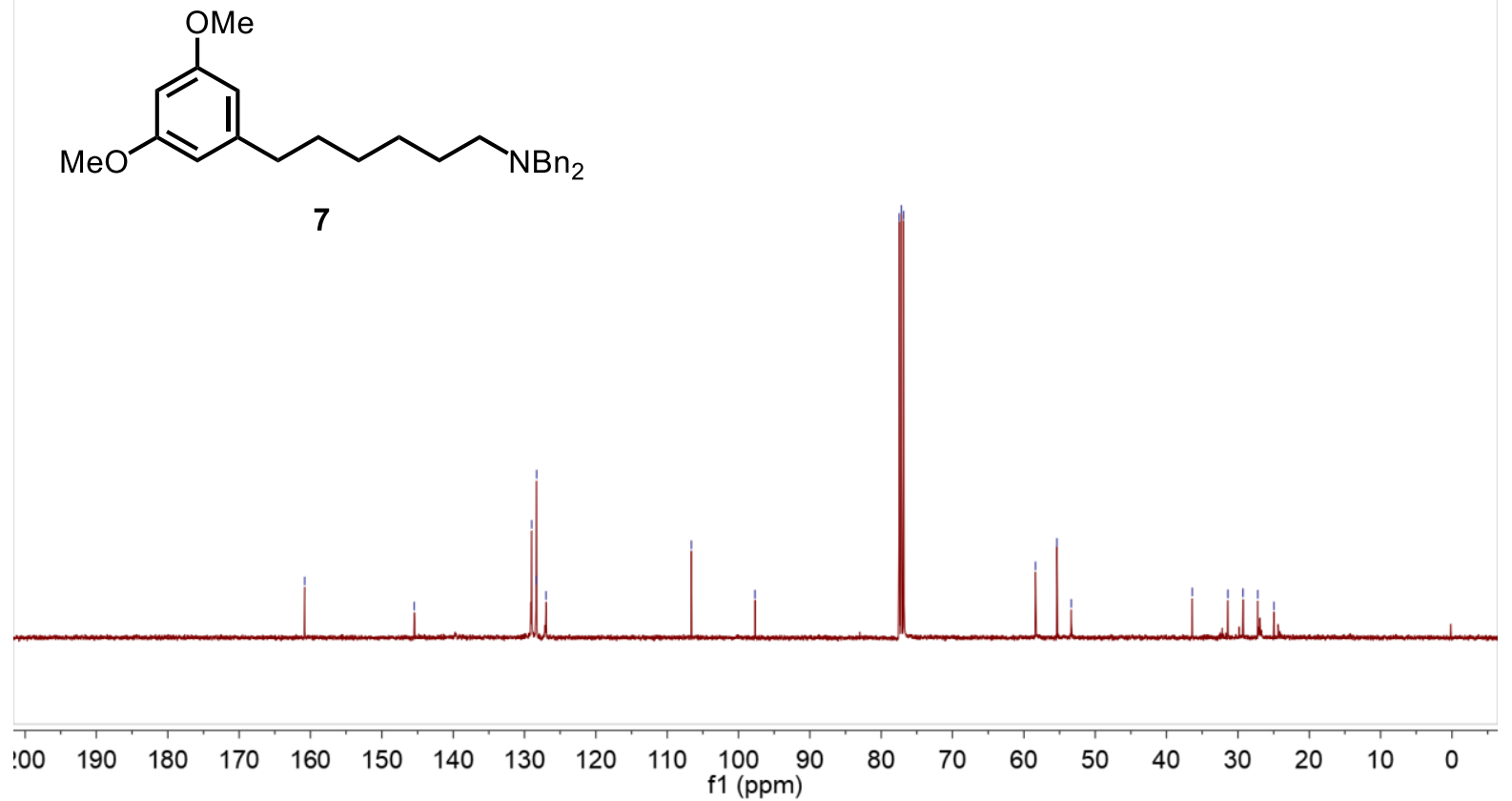

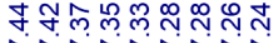

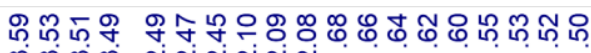

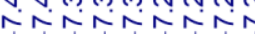

लुखm

${ }^{1} \mathrm{H}$ NMR (400 MHz, Actone)

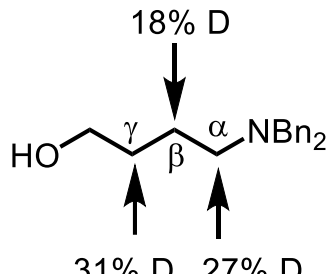

D-2b

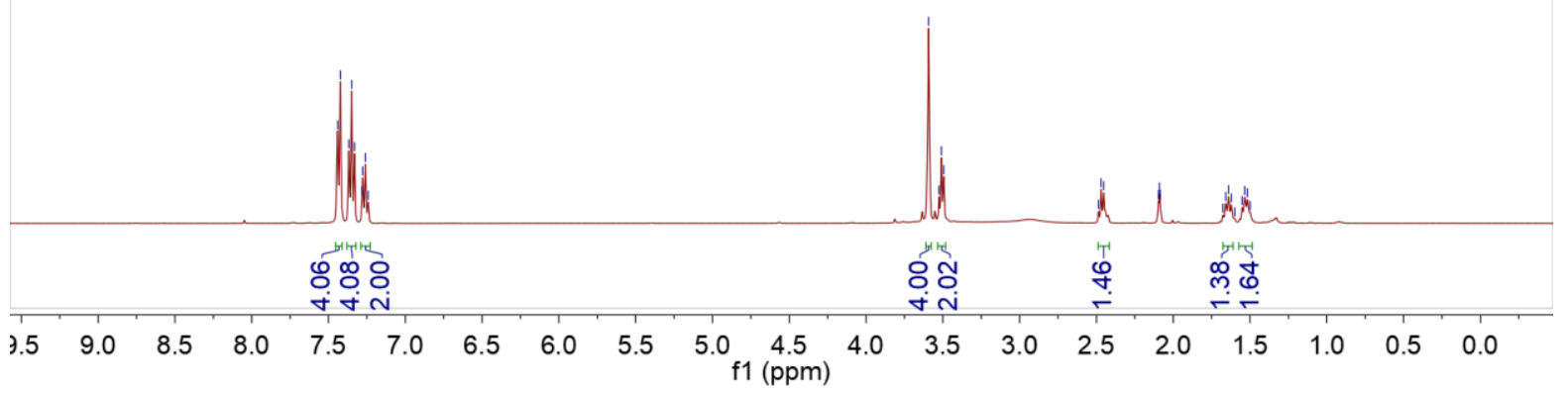




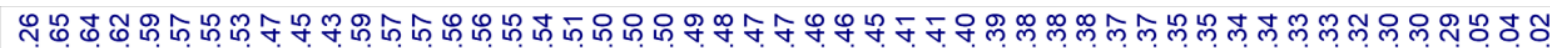

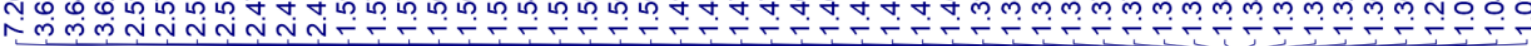

${ }^{1} \mathrm{H} \mathrm{NMR}\left(400 \mathrm{MHz}, \mathrm{CDCl}_{3}\right)$

$\sim_{\mathrm{NEt}_{2}}$

2ah

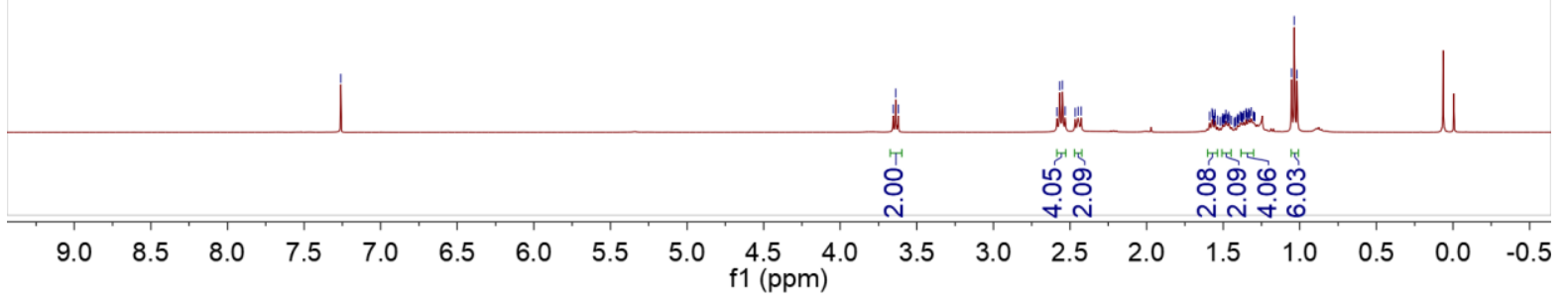

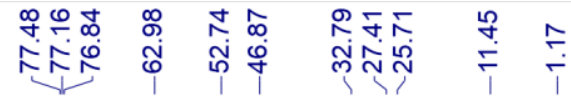

${ }^{13} \mathrm{C} \mathrm{NMR}\left(100 \mathrm{MHz}, \mathrm{CDCl}_{3}\right)$

$\sim_{\mathrm{NEt}_{2}}$

$2 \mathrm{ah}$

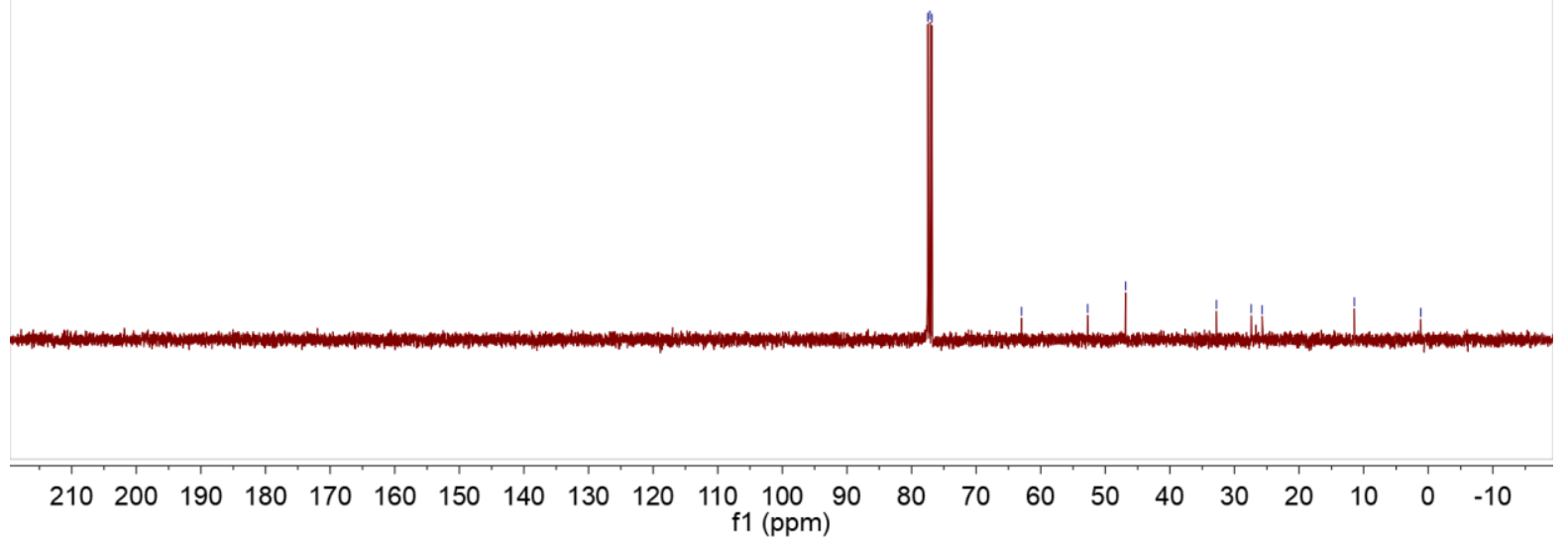

